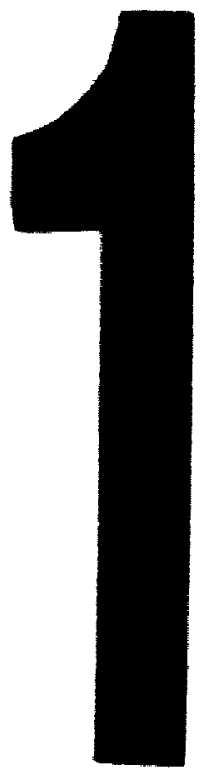

PM-1 31/2" " 4 " PHOTOGRAPHIC MICROCOPY TARGET NeS $1010 a$ ANSI/1SO \#2 EOUIVALENT

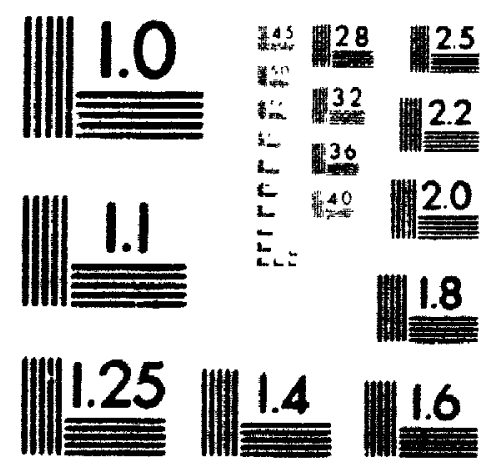


Acquisitions and

Bibliographic Services Branch

395 Weltington Street

Ottawa. Untaro

KIA ON4
The quality of this microform is heavily dependent upon the quality of the original thesis submitted for microfilming. Every effort has been made to ensure the highest quality of reproduction possible.

If pages are missing, contact the university which granted the degree.

Some pages may have indistinct print especially if the original pages were typed with a poor typewriter ribbon or if the university sent us an inferior photocopy.
La qualité de cette microforme dépend grandement de la qualité de la thèse soumise au microfilmage. Nous avons tout fait pour assurer une qualité supérieure de reproductior.

S'il manque des pages, veuillez communiquer avec l'université qui a conféré le grade.

La qualité d'impression de certaines pages peut laisser à désirer, surtout si les pages originales ont été dactylographiées à l'aide d'un ruban usé ou si l'université nous a fait parvenir une photocopie de qualité inférieure.

La reproduction, même partielle, de cette microforme est soumise à la Loi canadienne sur le droit d'auteur, SRC 1970, c. C-30, et ses amendements subséquents.
Reproduction in full or in part of this microform is governed by the Canadian Copyright Act,

R.S.C. 1970, c. C-30, and subsequent amendments. 


\title{
Integrating Automata with Genetic Algorithms in order to provide Adaptive Operators
}

\author{
by \\ Anthony R.P. White \\ A thesis submitted to \\ the Faculty of .duate Studies and Research \\ in partial fulfillment of the requirements for the degree of \\ Master of Computer Science
}

Ottawa-Carleton Institute for Computer Science

School of Computer Science

Carleton University

Ottawa, Ontario

May 12.1993

(c) Copyright 1993, Anthony White 
The author has granted an irrevocable non-exclusive licence allowing the National Library of Canada to reproduce, loan, distribute or sell copies of his/her thesis by any means and in any form or format, making this thesis available to interested persons.
L'auteur a accordé une licence irrévocable et non exclusive permettant à la Bibliothèque nationale du Canada de reproduire, prêter, distribuer ou vendre des copies de sa thèse de quelque manière et sous quelque forme que ce soit pour mettre des exemplaires de cette thèse à la disposition des personnes intéressées.

L'auteur conserve la propriété du droit d'auteur qui protège sa thèse. Ni la thèse ni des extraits substantiels de celle-ci ne doivent être imprimés ou autrement reproduits sans son autorisation. 


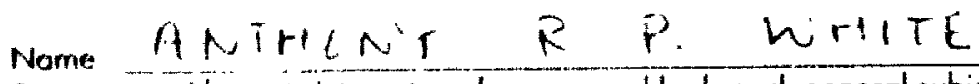

Dissertution Abstracts International is arranged by broad. general subject categories. Please select the one subject which most nesarly describes the content of your dissertation Enler the corresponding four-digit code in the spoces provided.

$$
\text { CUMUTER SCIENCE }
$$

\section{Subject Cotegories}

\section{THE mUMANITIES AND SOCIAL SCIENCES}

\begin{tabular}{|c|c|}
\hline 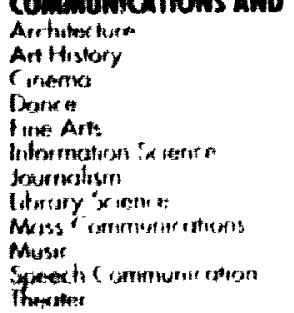 & $\begin{array}{l}0727 \\
0377 \\
0900 \\
0778 \\
0,37 \\
0123 \\
0391 \\
0349 \\
0708 \\
0417 \\
0454 \\
0465\end{array}$ \\
\hline 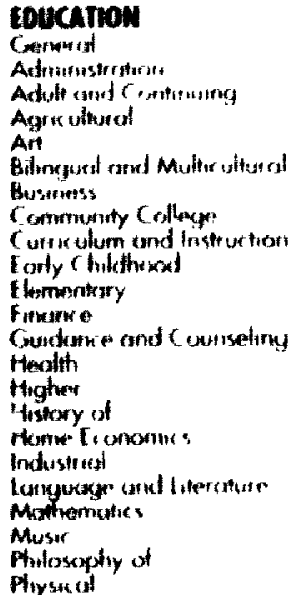 & $\begin{array}{l}0515 \\
0511 \\
0,16 \\
0517 \\
0,73 \\
0,87 \\
0688 \\
0275 \\
0727 \\
0518 \\
0 \% 24 \\
0277 \\
0514 \\
0680 \\
0745 \\
0520 \\
0278 \\
0421 \\
01278 \\
0280 \\
0522 \\
0596 \\
0523\end{array}$ \\
\hline
\end{tabular}

\begin{tabular}{|c|}
\hline 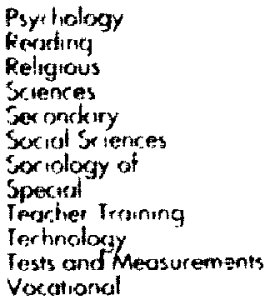 \\
\hline
\end{tabular}

\section{LAMGUAGE, LITRATURE AND UAMGUISTIES}

lonquerge

obeneral

$\begin{array}{ll}\text { Ancistit } & 0289 \\ \text { inguisfirs } & 0290\end{array}$

Modern

Literature:

Cenerd 040

Classical 0294

Comporative 0295

Medievol 0297

Medievol

African

Antericon

Cringenon (Engish)

Conodion (french)

tinglish

Comingme

Lotin Amerteon

Middle Fastern

Rommance

Slowir and East European
THEOLOGY

Phalosophy

Reliqion

Beneral

Clergy

History of

Philosophy of

Theology

soanl saructs

Americon Studies

Antriopology

Archoeology
Culfural

Physiral

Business Administrotion

Gener of

Acrounting

Bonking

Manogement

Conodian Studies

Economics

General

Ayricultural

finonce

History

Labor

Folklore

Geogrophy

Gerontology

History

$$
\text { General }
$$

0422

0318

0321
0319

0320

0322

0469

0.323

0324

0326

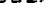

0310

0272

0770

0454

0385

0501

0503

0505
0508

0509

0510

0511

$0^{-} 3$
0.6

0351

0578

\begin{tabular}{|c|c|}
\hline $\begin{array}{l}\text { Ancient } \\
\text { Medievol } \\
\text { Modern } \\
\text { Block } \\
\text { Africon } \\
\text { Asio, Austrolio and } \\
\text { Conodion } \\
\text { European } \\
\text { Lotin American } \\
\text { Middle Eustern } \\
\text { United States } \\
\text { tory of Science }\end{array}$ & , \\
\hline
\end{tabular}

SUDJeCT CODE
Law

Pótical Science

Genero:

International Low and

Relotions

Public Administrotion $\quad 0617$

Recreation. , . 0814

Socid Work

Sociology

Generol 0626

Crrminology and Penology 0627

$\begin{array}{ll}\text { Demogrophy } & 0938 \\ \text { Ethnic and Rociol Studies } & 0631\end{array}$

individual and fomily

Studres

Industrial and labor

Relations.

0628

Social Structure and

Develop:nent

Theory and Methods

Transportation 0709

Wran and Regional Planning 099

0629

0630

0700

0344

0453
Public and Social Wellore

THE SCIENCES AND ENGINEERINO

\section{mockat satices}

Aynicuture,

Atponisny

Nulitions

Animal Pothotioy

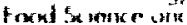

lextansicaly

forestry oms withit.

Fum Cutwe

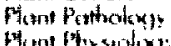

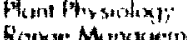

Wexd Texhology

Rrotogy

int?

Arubionaty

Thistins

(teill

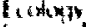

thermoling

(t)intetis

Morinderitery

Molkt tho

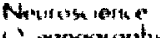

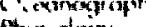

Ruthothot

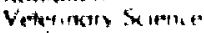

indexty

Bugnitys

Caner of

Emtu satencs

fingers lawert sto
Armond Cultute and

iench lewester
Crooktosy

Geophys

Geophysics
Hydiology

Miner clogay

Palentrotaity

Polecer ology

Poleozooligy

Paivinesiogy

Physical Geogrophy

Phrsical Oceonography

\section{nEATU ANO EnVIROMUENA}

\section{setwas}

Fintominemter: Sorences

Hexith Screm es

Crener ol

Audiology

chemether opy

Dentistry

Iducotion

Hospend Manogennent

Himant Developifuert

Immintshogy.

Murite ine and Sugery

Mervtal health

Nising

Nutition

Obstetics and Gynecology

Or cupcilionis thealti and

Theroory

Qphtionoloyy

Prithologr.

Pilamocoludy

Pharmoky

Physad theropy

Public Heglin

Ratiology
0370

0372

0373

$038 \mathrm{~B}$

0411
0345

0426

0418

0985

042

0368
0415

415

\footnotetext{
Speech Pothology

Toxicology
}

\section{PHYSFCAL SCAENCES}

Pure Sciences

Chemistry

Generd
Agricultura

Anolytical

Bochemistry

inorgonis

Nuclear

Orgonic

Physical

Polymer

Rodiation

Mathematics

Phystes

Generd

Acoustics

Astronomy and

Astroptiysics

Atrinesphetic Science

Atomic

Elementory Particles and

High Energy

Molecular

Nucteor

Optess

Rodiotion

Solid Stote

Statistics

Apitind Scionces

Aoplred Mechanics
Electronics and Electricity
0460

0386

Engineering

Aerospace

Agricultural

Automolive

Chemica!

Civil

Electrinics and Electricat

Heat and Thermodynamics

Hyóroulic

industrim

Marine

Materigls Scrence

Mechonical

Metallurgy

Mining

Nucleor

Packoging

Santary and Municipal

System Scrence

Geotechnology

Operations Research

Plastics Jechnology

Textile Technology

\section{Pronoter}

Generol

Behaviora

Cinical

Developmental

Experiment

Personality

Physiological

isychobiology

Psychometrics

0346
0537 
The undersigned hereby recommend to the Faculty of Graduate Studies and Research acceptance of this thesis.

Integrating Genetic Algorithms with Automata in order to provide Adaptive Operators submitted by Anthony R.P. White. M.A.

$A$ thesis submitted

in partial fulfillment of the requirements for the degree of Master of Computer Science

i'

Thesis Supervisor

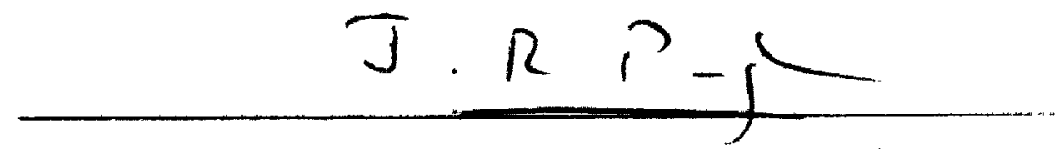

Chairman, School of Computer Science

\section{Carleton University}

May 12. 1993 


\section{Abstract}

Nature has conspired to cause change over time as a result of a changing environment. Life has shown itself to be highly successful in exploiting the characteristics of one generation and improving from one generation to the next. This process of environmental ixploitation. called Evolution, has been described and studied using Genetics.

Genetic Algorithms are based loosely upon the principles of survival of the fittest and exploitation of genetic material from one generation to the next. Genetic Algorithms have shown themselves to be successful in solving a wide range of combinatorial problems. (ienetic Algcrithms have traditionally required the specification of a number of parameters that control the evolutionary process; two of these being related to the probability of applying the mutation and crossover operators.

This thesis proposes extensions to the classical Genetic Algorithm model. In the classical modkl. the aforementioned mutation and crossover operator probabilities are specified hefore the start of a Genetic Algorithm run and remain unchanged, a so-called static model. This thesis uses automata in order to allow the adaptation of these operator probabilities as the run progresses in order to improve quality of the solutions found. Favourahle results have been achieved for a wide range of function minimization problems and these are described. 


\section{Acknowledgments}

I would like to express my gratitude to Prof. Optasher for the time he has take'" in reviewing, and commenting on. this thesis and for providin: the valuable insights that enabled me to complete this work. I would also like to thank him for his teithing in various courses that I have taken over the span of this Masters degres. These coum $x-1$ have no doubt - prepared me for the thesis itself.

I would also like to acknowledge the contribution of Prof. Oommen's Naching in the area of Automata Theory - material at the very core of this thesis.

Finally, but by no means least. I would like to thank my wife Nikhi: without whose support. understanding and constant encouragement, very little would Ixpossible. 


\section{Table of Contents}

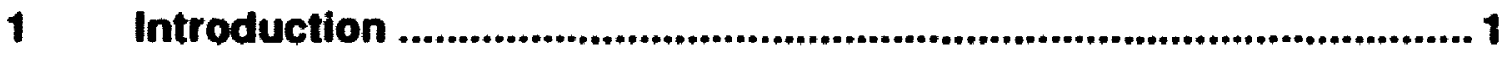

1.1 Organization of this thesis ................................................. 4

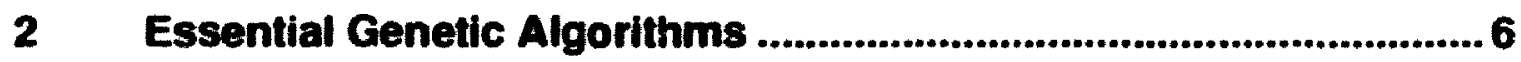

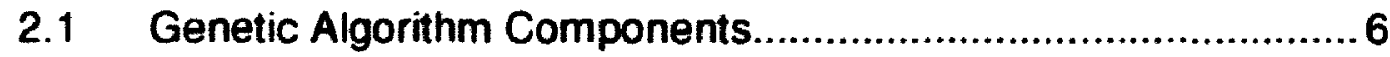

2.1.1 initializePopulation ............................................... 10

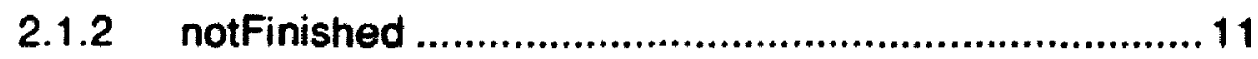

2.1.3 evaluatePopulationFitness .....................................11

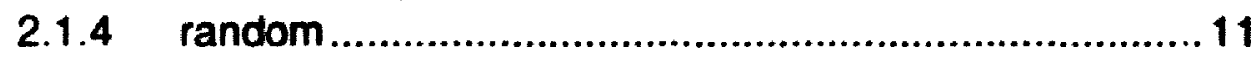

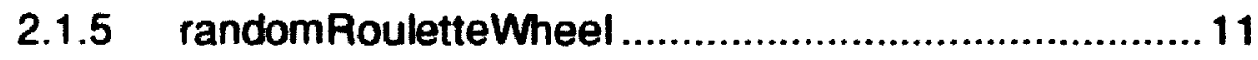

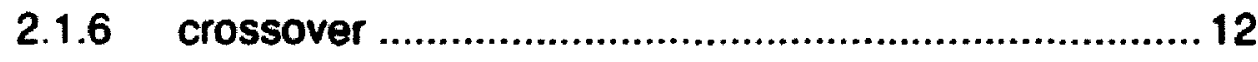

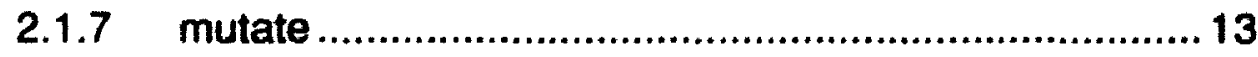

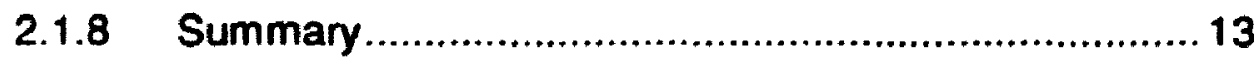

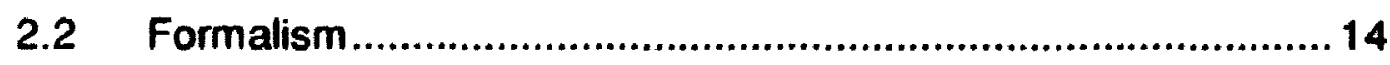

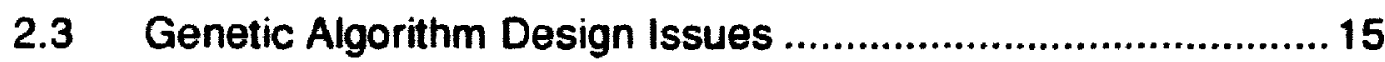

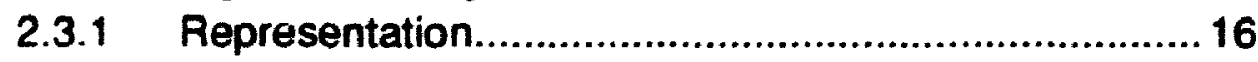

2.3.1.1 Alphabet Choice .......................................... 16

2.3.1.2 Encoding Strategy .................................... 18

2.3.2 Reproductive Scheme.........................................20

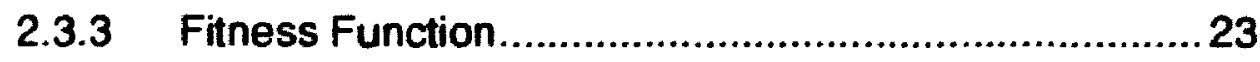

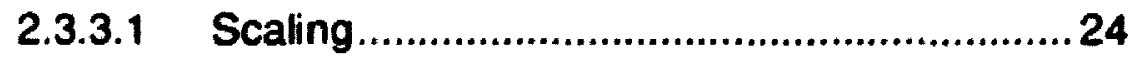

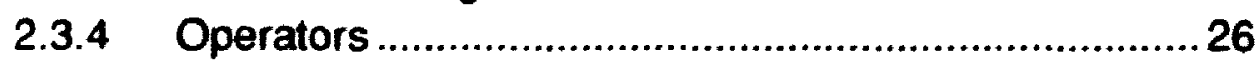

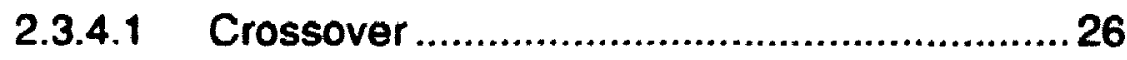

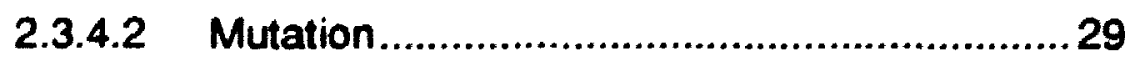

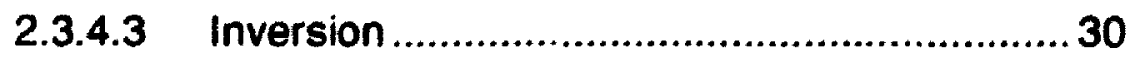

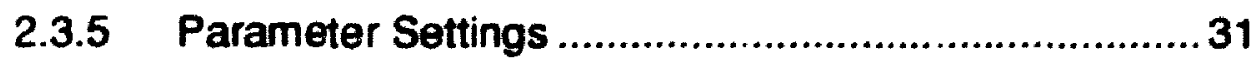

3 Overview of Genetic Algorithms .................................................33

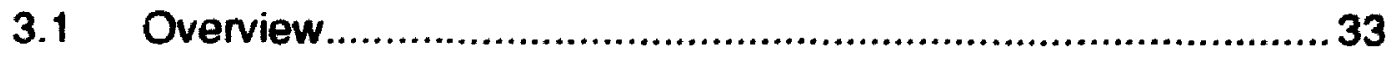




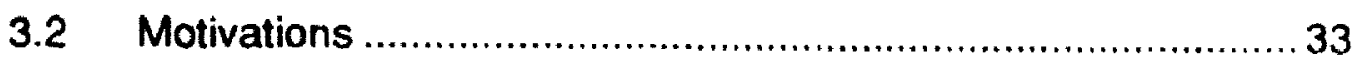

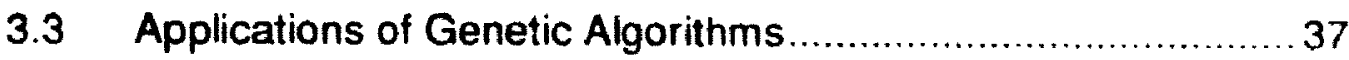

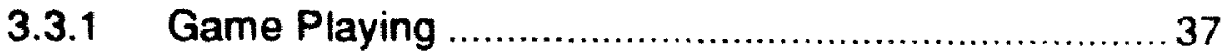

3.3.2 Function Optimization ...........................................44

3.3.3 Neural Network Optimization ................................ 47

3.3.3.1 The Static Problem ....................................49

3.3.3.2 The Dynamic Problem ................................ 51

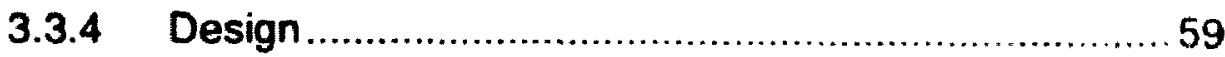

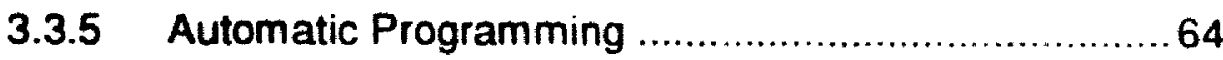

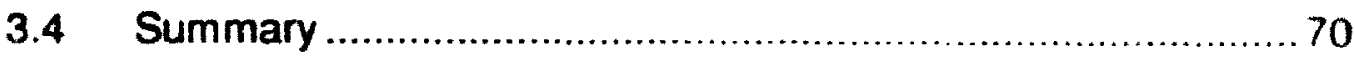

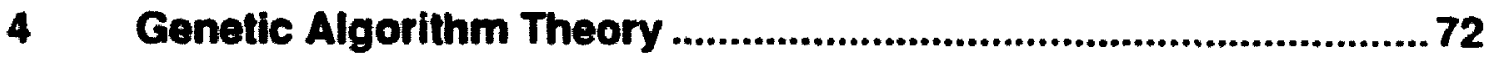

4.1 The Schema Theorem...................................................... 72

4.2 Schema Disruption and Combination ...................................79

5 Genelic Algorithms and other Optimization techniques ...............82

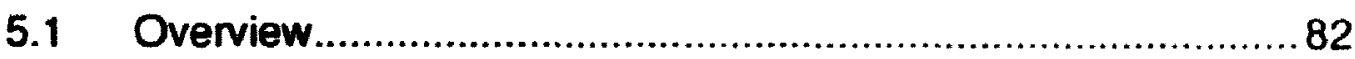

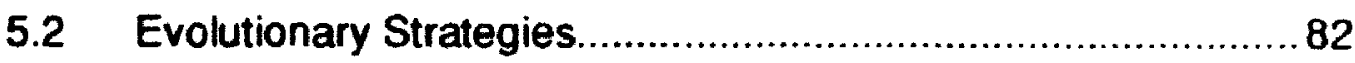

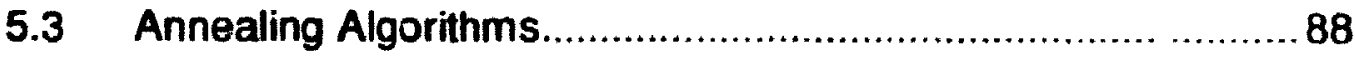

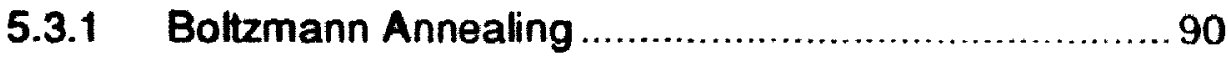

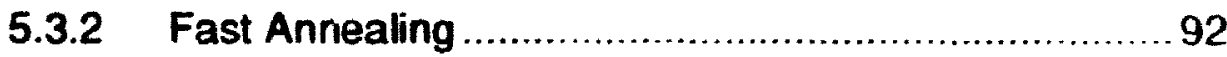

5.3.3 Very Fast Annealing ............................................. 92

6 Adaptive Genetic Algorithms ......................................................96

6.1 Convergence control ......................................................96

6.1.1 Ranking and Adaptive Population Size ....................97

6.1.2 Diversity Maintenance using Entropy .......................99

6.2 Punctuated Crossover .................................................... 103

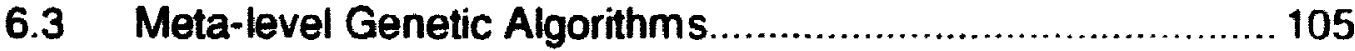

6.4 Adaptive Operator Selection........................................ 106

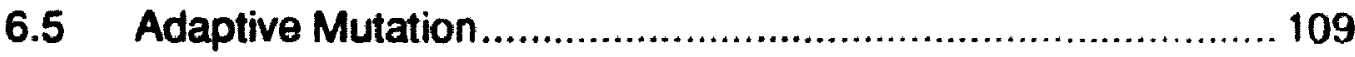

$7 \quad$ Automata Theory ............................................................................ 112

8 Adaptive Crossover Using Automata...................................... 118

8.1 The adaptive uniform crossover operator ........................... 119

8.2 The adaptive multi-point crossover operator ..................... 123

8.3 Experimental setup and results overview ........................... 124

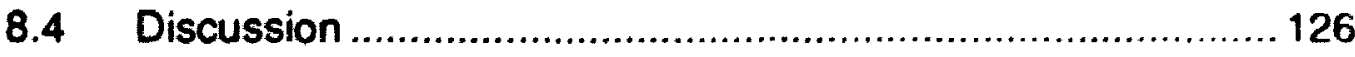

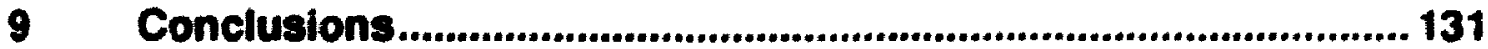

9.1 Direction Of Future Work .............................................. 133 
9.1.1 Automata Investigations............................... 133

9.1.2 Parameter Investigations .................................... 134

9.1 .3 Theoretical Study ........................................... 134

9.1.4 Operator Investigations ..................................... 134

9.1.5 Integration with other Adaptive Operators .............. 135

9.16 Application to Genetic Programming ................... 135

10 References ............................................................................. 136 


\section{List Of Figures}

Figure 1

Figure 2

Figure 3

Figure 4

Figure 5

Figure 6

Figure 7

Figure 8

Figure 9

Figure 10

Figure 11

Figure 12

Figure 13

Figure 14

Figure 15

Figure 16

Figure 17

Figure 18

Figure 19

Figure 20

Figure 21

Figure 22

Figure 23

Figure 24

Figure 25

Figure 26

Figure 27

Figure 28

Figure 29

Figure 30

Figure 31

Figure 32
Examples of schema 9

Fopulation Roulette Wheel ............................................... 12

Crossover in action....................................................... 13

Binary coding example ........................................................ 18

Gray coding example....................................................... 19

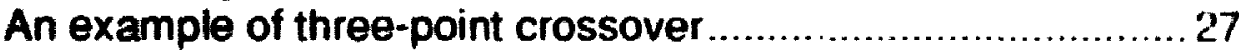

An example of uniform crossover operator ......................... 28

$f_{5}$ performance for varying uniform crossover probability ........29

An example of the inversion operator .................................30

Genetic Algorithm Design of Neural Networks .....................49

Crossover on real-valued boundaries.................................. 50

Overview of neural network bit string representation .............. 51

Area and Projection Specification field detail .......................52

Demonstration of Graph Generation Grammar for XOR ......... 55

Graph generation rules for 2-2-1 XOR network...................... 56

Chromosome encoding grammar.......................................56

Genetic Programming and Neural Networks ...................... 58

The Genetic Algorithm Primary Neighbor selection process ... 60

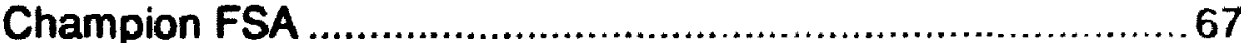

Genetic Programming Crossover Operation .........................69

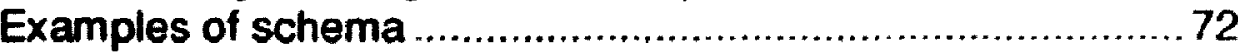

Fitness landscape of a typical function ............................... 89

Plot of the Diversity Function ........................................ 100

Mutation Probability as a Function of Diversity.................. 102

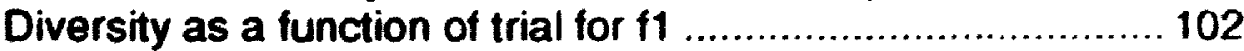

Punctuated crossover bit string representation ................... 104

Meta Genetic Algorithm Architecture ............................... 105

:Schematic of mechanism used for adaptive mutation ...........111

Automaton interaction with its environment ...................... 112

Schematic of adaptive uniform crossover operator .............. 119

Automaton reward-penal.y scheme.................................. 121

$f_{3}$ difference between non-adaptive and adaptive runs ......... 127 
Figure 33

Figure 34

Figure 35

Figure 36

Figure 37

Figure 38

Figure 39

Figure 40

Figure 41

Figure 42

Figure 43

Figure 44

Figure 45

Figure 46

Figure 47

Figure 48

Figure 49

Figure 50

Figure 51

Figure 52

Figure 53

Figure 54

Figure 55

Figure 56

Figure 57

Figure 58

Figure 59

Figure 60

Figure 61

Figure 62

Figure 63

Figure 64

Figure 65

Figure 66

Figure 67

Figure 68

Figure 69

Figure 70

Figure 71

Figure 72

Figure 73

Figure 74

Figure 75

Figure 76

Figure 77

$f_{6}$ difference between non-adaptive and adaptive runs 128

$f_{10}$ difference between non-adaptive and adaptive runs ....... 129

11 non-adaptive fitness 164

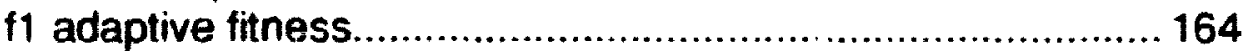

f1 adaptive - non-adaptive comparison .............................. 165

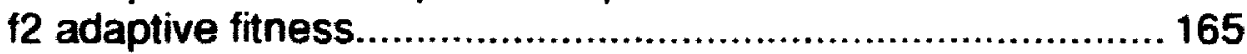

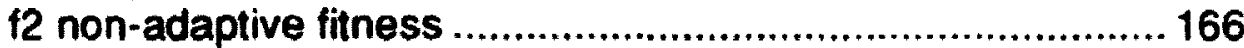

$\{2$ adaptive - non-adaptive comparison .............................. 166

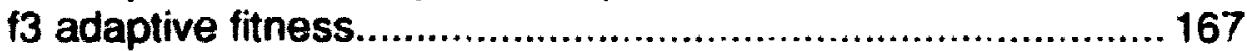

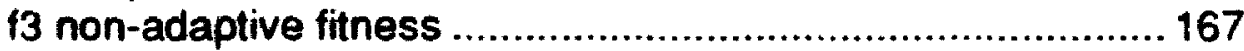

13 adaptive - non-adaptive comparison .............................. 168

14 non-adaptive fitness .................................................. 168

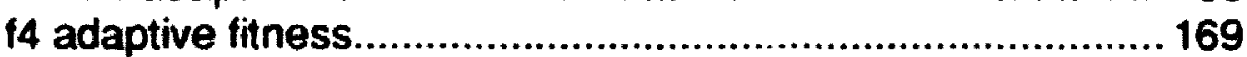

f4 adaptive-non-adaptive comparison ................................. 169

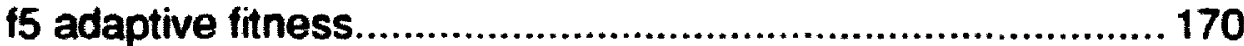

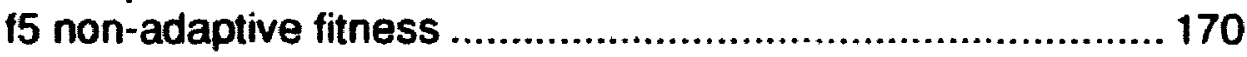

f5 adaptive - non-adaptive comparison .............................. 171

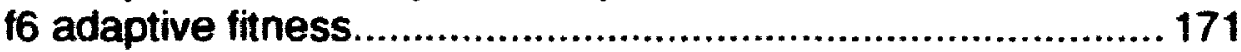

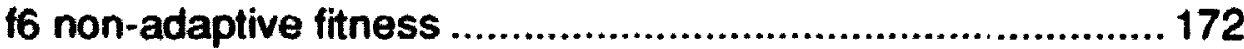

t6 adaptive - non-adaptive comparison ............................... 172

17 non-adaptice fitness ......................................................173

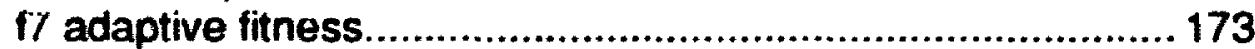

17 adaptive - non-adaptive comparison ............................. 174

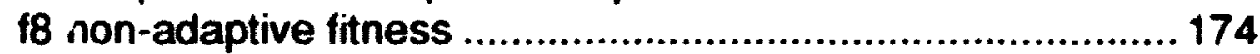

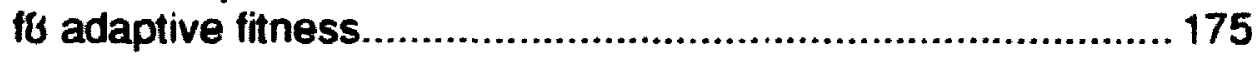

18 adaptive - non-adaptive comparison ............................. 175

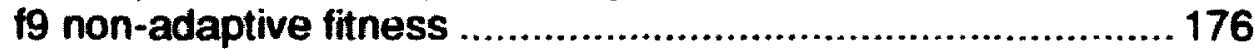

t9 adaptive fitness............................................................176

19 adaptive - non-adaptive comparison ............................ 177

f10 non-adaptive fitness ................................................ 177

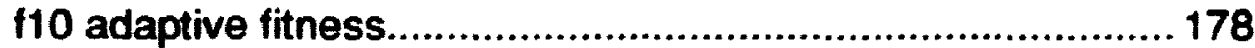

f10 adaptive - non-adaptive comparison ............................ 178

111 non-adaptive fitness.................................................. 179

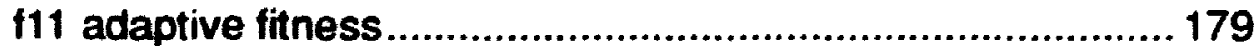

f11 adaptive - non-adaptive comparison............................ 180

$\mathbf{1 1 2}$ non-adaptive fitness ................................................ 181

$\mathrm{f12}$ adaptive fitness..................................................... 181

f12 adaptive - non-adaptive comparison ........................... 182

f13 non-adaptive fitnes ............................................... 182

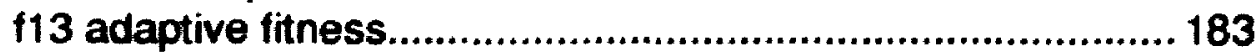

f13 adaptive - non-adaptive comparison ............................ 183

f14 non-adaptive fitness .................................................. 184

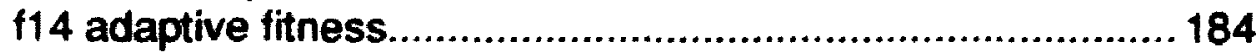

f14 adaptive - non-adaptive comparison .......................... 185

f15 non-adaptive fitness ................................................. 185 
Figure $78 \quad \mathbf{1 1 5}$ adaptive fitness............................................... 186

Figure $79 \quad \mathrm{f15}$ adaptive - non-adaptive comparison ....................... 186

Figure $80 \quad \mathbf{1 1 6}$ non-adaptive fitness ..................................... 187

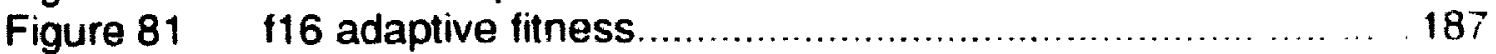

Figure $82 \quad \$ 16$ adaptive - non-adaptive comparison .................. 188

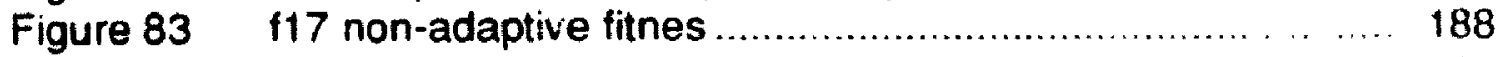

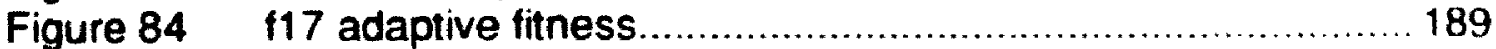

Figure $85 \quad \mathrm{f17}$ adaptive - non-adaptive comparison ..................... 189

Figure $86 \quad f 18$ non-adaptive fitness ............................................. 190

Figure $87 \quad f 18$ adaptive fitness..................................................... 190

Figure $88 \quad\{18$ adaptive - non-adaptive comparison .......................... 191

Figure $89 \quad f 19$ non-adaptive fitness ............................................... 191

Figure $90 \quad 119$ adaptive fitness........................................................ 192

Figure $91 \quad f 19$ adaptive - non-adaptive comparison ......................... 192

Figure $92 \quad\{20$ non-adaptive fitness ............................................ 193

Figure $93 \quad$ 20 adaptive fitness......................................................... 193

Figure 94 f20 adaptive - non-adaptive comparison ............................. 194

Figure 95 †21 non-adaptive fitness ........................................... 194

Figure $96 \quad\{21$ adaptive fitness.................................................... 195

Figure $97 \quad 21$ adaptive - non-adaptive comparison ........................... 195

Figure 98 t22 non-adaptive fitnass ............................................. 196

Figure 99 222 adaptive fitness................................................ 196

Figure 100 \{22 adaptive - non-adaptive comparison ......................... 197

Figure 101 t23 non-adaptive fitness ......................................... 197

Figure 102 t23 adaptive fitness.................................................. 198

Figure $103 \quad 23$ adaptive - non-adaptive comparison ........................... 198

Figure $104\{24$ non-adaptive fitness .............................................. 199

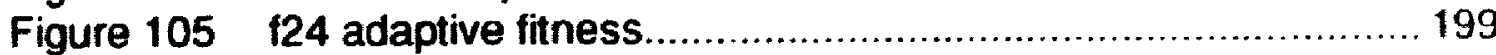

Figure 106 f24 adaptive - non-adaptive comparison .......................... 200 
Table 1 Table 2 Table 3

Table 4 Table 5 Table 6 Table 7 Table 8 Table 9

Table 10 Table 11 Table 12 Table 13 Table 14 Table 15 Table 16 Table 17 Table 18 Table 19
Binary and Gray Codes 19 Genetic algorithm parameter setting defaults 31

Payoff matrix for the Prisoner's Dilemma 39 Tower of Hanoi coding scheme 42 Selection schemes used in Hollstein's study ......................... 45 Mating methods used in Hollstein's study 45 DeJong's Reproductive plans ...............................................4 47 Graph Generation Scheme Symbols .................................. 55 Binary-coded State Transition Table ....................................66 Evolutionary strategy recombination operators .....................84 Fianking allocation function ..........................................97 Common experimental parameters ..................................125 Adaptive crossover operator parameters ........................... 126 Coefficients for $f_{18}$........................................................ 156

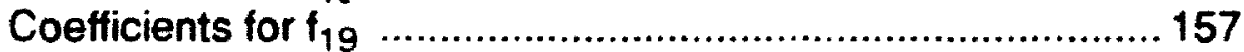

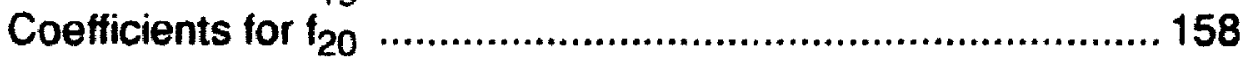

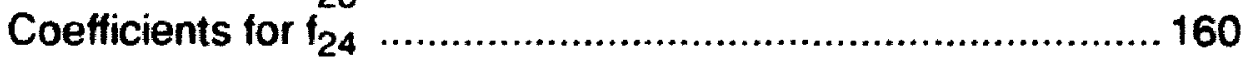
Common experimental parameters .................................. 162 Adaptive crossover operator parameters ......................... 163 


\section{Table of Appendices}

Test Functions

.150

Results 161 


\section{Introduction}

Life seems capable of solving incredibly complex problems. Consider, for example, the human mind. Evolved over a long time span, it constitutes an incredibly efficient general-purpose design; being able to store a life time of experiences and knowledge all in a small volume with enviable recall capabilities. It is inconceivable that such a device could have appeared purely as a result of chance. Its intricate complexity seems to defy the idea that a sequence of random choices led to the current design. Can rand'm processes be the only guiding force behind nature? Einstein certainly did not believe so when he said of Quantum Mechanics, "God does not play dice."

Biology is filled with examples of extraordinarily complex systems. The fertilization and development of the human fetus being amongst the most amazing. Here, thousands of genes regulate one another within individual cells causing an immune system to develop with remarkable adaptive properties and billions of neurons to be massively interconnected in networks which themselves possess learning behaviours ultimately allowing a child to master, and improve upon, the world in which he lives.

Again. it does not seem possible that these highly ordered systems have evolved by randomly changing the structure, or programming, of the previous generation. These intricate structures are maintained and duplicated through a precise balan ie of chemical and hehavioral activities with a single element out of place causing degradation in the behaviour of the overall system. However, the keyword is structured, or 
rather hierarchical. These systems did not immediately coalesce from a soup of genetic material but rather formed nuclei. with subsystems initially developing. The nuclei in turn forming clusters of nuclei; followed by clusters of clusters until we reach the levels of complexity that we see today.

What caused such order to develop: Since Darwin, hiologists have scc'n natural selection as almost the only source of the order in evolution. Ilowever, Darwin could scarcely have suspected of the existence of self-organization: a recontly discovered, innate property of some complex systems |kiaffiman. |W(9)|. II is considereci possible that biological order partially reflects the spontaneous order on which selection has acted. Selection has molded the native colberence of ontogeny, or biological development. even though it was not compelled 10 invent it. It is possible that the capacity to adapt and erolve is itself an adhicvement of evolution.

To understand how self-organization can the a force in evolution, the study and use of complex systems is necessary. Complex systems often exhibit the phenomenon known as "chaos". Chaos allows dynamic, non-linear systems that are orderly at first to become highly disordered over time with different starting conditions causing the system to behave in wildly varying ways. Equally mossibls is that certuin disordered systems "(rystallize" into highly ordered systems. Antichaos, it is believed, plays an important part in biological development and evolution. The self-regulating network of the genome (the complete set of genes in an organism) offers a good example of a system where antichaos may govern development. In fact, the interaction of Artificial Intelligence and (haos Theory has recently been discussed in |Kiss, 199||.

Natural systems have often provided the inspiration for successful problem solving algorithms and this thesis is founded on one such analogy - the simulation of evolution using the genetic algorithm. Genetic algorithms draw upon the work of Darwitt, and the study of Genetics. The genetic algorithm, due to Holland, is an example of a complex, non-linear system where an initially disordered system crystallizes to a highly ordered state through the mechanisms of sexual reproduction, explositution and survival of 
the fittest. Examples of both of these latter characteristics are available in abundance in Nature. Man has shown himself to be particularly adept at exploitation.

Cienetic algorithms belong to the class of "weak" algorithms where the algorithm operates on a representation of the problem in question with no encoded domain knowledge ${ }^{l}$;uch as is the case in Explanation Based Learning. Further, genetic algorithms work with an encoding of the parameters of the problem rather than the parameters themselves and sexual reproduction is simulated by the exchange of genetic information using an operator which combines genetic material from the parents; redistributing it in the offspring. In Nature, the success of an individual is most usually measured by the ability to reproduce, having avoided predation. In the genetic algorithm, this is replaced by a function - called the fitness function - which is assumed to map onto the solution space.

The redistribution of genetic material in Nature, or rather the expression of features derived from the genetic encoding, is a dynamic process. Here, in one generation a particular encoding may result in a feature value $X$ whereas in a subsequent generation the result may be $Y$. This arises as a result of the continual change in the dominance and recessiveness of alleles within the chromosome. In a Holland genetic algorithm, the redistribution process is static in that a successful redistribution of genetic material is not noted in such a way that future redistributions will fend to keep those genes together.

This thesis attempts to make the redistribution process adaptive by noting successful (and unsuccessful) redistributions of genetic material - rewarding success and penalizing failure. By doing this. the genetic al gorithm becomes more like an evolutionary strategy |Schwefel. 1975], where two level learning is supported. This thesis draws together two pieces of previous work. Firstly, it builds upon the work of Syswerda; whose uniform crossover operator has been shown to be successful in a nu. ber of problem environments, despite the limitation noted above. Secondly, it uses Variable State Stochastic Automata - due to Oommen - in order to provide the adaptive redistribution of genetic material. This combination of mechanisms provides a more accurate analogy to

1. If is arguable that the fintess function consitures domain knowledge. 
the process embodied in Nature and is shown to be more effective than the static algorithm previously described.

\subsection{Organization of this thesis}

The overall aim of this thesis is to take the reader from no knowledge of gentic algorithms through to having sufficient knowledge to use these technic|ues in sulving real optimization problems. This author hopes that by reading this dicument the reader will come to appreciate the power of evolutionary algorithms, such as the genetic algorithm.

This thesis is composed of nine chapters. Chapter I is this introduction. (hapler I describes the genetic algonthm in depth and consists of three subsections. It de'sirile's the genetic algorithm from a mechanical standpoint, starting with a sectlon describing the classical genetic algorithm followed by a section that describes the design decisions. and problems which have to solved when employing the genetic algorithms. The thre subsections of this chapter provide essential definitions and dexcribe the statc of rescarch in the areas of population selection techniques. problem representation. fitmess funclion construction, operator specification and parameter selection. Chapter 3 provicles an overview of genetic algorithm; elaborating on the biological metaphor and unlertake's a survey of the wide range of applications that have been described in the literature. ( 'hapter 4 undertakes an exploration of the theory underlying genetic algorithms, starting with the conventional schema analysis and ending with the theory of schema disruption and combination. Chapter 5 contrasts the genetic algorithm with other optimisation techniques. It compares, in some detail, very fast simulated annealing with genetic algorithms. This chapter also discusses the similarities and differences between gemetic algorithms and evolutionary strategies. Chapter 6 surveys previous work that has lxe'l undertaken in the area of adaptive operator probabilities in the context of fenctic algorithms, highlighting both strengths and weaknesses of the approaches chosen. ( hapter 7 introduces Automata theory, providing definitions, essential background theory and a description of Variable State Stochastic Automata (VSSA). (hapter $\times$ brings tegcther genetic algorithms and VSSA in a framework which allows adaptivi operalos probabilities and introduces the adaptive uniform crossover operator and prexents the results of using the adaptive crossover operator on an extended test bed of mathematical 
functions. Finally, chapter ${ }^{9}$ puts forward conclusions reached as a result of the study and proposes work whicll might be undertaken in order to extend the work and to justify the use of the adaptive uniform crossover operator in wider domains. It briefly describes how a operator similar in concept to the adaptive uniform crossover operator could be used to enhance the recently introduced genetic programming paradigm |Koza, 1990| with a view to automating the process of function definition. 


\section{Essential Genetic Algorithms}

This chapter is composed of two distinct sections. It is designted In provile at working knowledge of the genetic algorithm and its components. followed by a detailed description of issues arising from genetic al gorithms.

\subsection{Genetic Algorithm Components}

In general, an abstract task can be thought of as being a problem the solution to which can be found by searching some abstract space of solutions /Michadski. 1983]. As we are usually asking for the "best" solution, the task can be viewed as an optimization process [Holland, 1975].

Genetic algorithms (GAs) were originally proposed as a search technique for use in optimization problems. Many optimization problems are hard and although in specific cases algorithms of considerable importance have been developed for them, they are quite often not able to guarantee an optimal solution. Examples are the use of Simulated Annealing for wire routing and component placement problems [Kirkpatrick et al, 19831. General techniques for the solution of NP. hard problems have not been forthcoming.

Genetic algorithms are stochastic search algorithms whose search methoxh lokklel aspects of Darwinian evolution. As stated in [Davis, Steenstrup. 1987]:

"... the metaphor underlying genetic algorithms is that of natural evolution. In evolution, the problem each species faces is one of searching for beneficial adaptations to a 
complicated and changing environment. The 'knowledge' that each species has gained is embodied in the makeup of the chromosomes of its members."

The basic principle behind genetic algorithms is to do what Nature does. The fitter the individual, the more likely it is to survive to be able to reproduce and thereby ensure that its genes survive to the next generation. While this section provides a brief introduction to genetic algorithms, more complete descriptions can be found in [Goldberg. 198') and |Michalewicz, 1992].

Genetic algorithms use a vocabulary drawn from natural genetics. We talk about individuals (or genotypes) in a population. These individuals are sometimes also referred to as chromosomes or strings. Having individual and chromosome as synonyms in this context is a little confusing in that in man, for example, an individual is made of cells each of which contain $\mathbf{4 6}$ chromosomes. In genetic algorithms, a population consists of a set of single chromosomes. Chromosomes are made up of units called genes or feurures most often arranged in a linear formation or string. A feature is just a set of genes. Each gene controls the inheritance of one or more traits. The genes are said to express a given feature. Geres of certain features are located at specific locations on the chromosome - these are called loci or string positions. A feature which can have several values means that the associated genes can take on many values. The values which a gene can take are called alleles.

In the context of this thesis, each chromosome would represent a potential solution to an optimization problem. An evolution process run on a population of such chromosomes corresponds to a search through a space of potential solutions. The search process requires balancing two conflicting objectives: exploiting the best solutions at any point during the process and exploring the search space. Hill climbing is an example of a strategy which retains as much of the hest solution to date as possible when moving to the next solution - only suall perturbations are made and the results retained only if improvement is seren. Consequently, it neglects the exploration of the search space and only guarantees to bblain an optinual solution if the solution space contains a single perak. Random search, on the other hand. explores the solution space without 
regard to the structure of the best solution to date nor to the region of the spatce where it was obtained. Genetic algorithms combine hoth solution explottation and exploration of the search space in an extremely effective way.

Genetic algorithms have been used in a diverse set of optimization problems such as wire routing. travelling salesman problems, network link devign /C'omuls. Davis, 1987), |Davis, Coombs, 1987|, adaptive control and many others. (onsult [Michalewicz, 1992] for more details on optimization problems in the areas of scheduling. strategy fomiulation and transportation problems. Chapter 3 provides a description of a number of applications of genetic algorithms in the areas of Game Playing. Function Optimization, Neural Network Optimization, Design and Automatic Programming.

Genetic algorithms differ from more convectional search algorithms in that they work with a population of chromosomes (solutions) rather than a single chromossome (solution). Genetic algorithms also work indirectly, in that problem parameters are emcikded lxefore being manipulated by genetic operators. Finally genetic algorithms differ from most conventional search strategies in that they use probabilistic transition rules. not deterministic rules. As such they belong to the class of stochastic search al gorithins.

Restricting the discussion to chromosomes represented as bit strings, each chromosome is the same length and each gene can take values drawn from $\{11.1\}$ - the so called hillary al phabet. In this case, the chromosome is represented by a bit string and for a chromosome of length 1, $2^{1}$ states can be represented. Generally, for an alphabet of cardinality $\mathrm{m}, \mathrm{m}^{\prime}$ states can be represented ${ }^{1}$.

Genetic algorithms are often characterized as algorithms that are better than hill climbing algorithms because they manipulate combinations of bit strings called schematia ${ }^{2}$. It we consider the binary alphabet for the moment, the al phabet of associated schemata would

1. The principle of minimum alphathets states that we should use the smallest alphathel which intakes semse

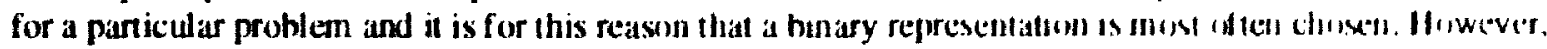

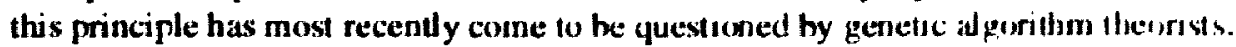

2. The terms schemala and schema are used interchangeably. 
he $\{1,1, \#\}$, where the \# symbol indicates unknown. Figure 1 shows a shema and two possible strings which are examples of it. A schema represents a class of strings.

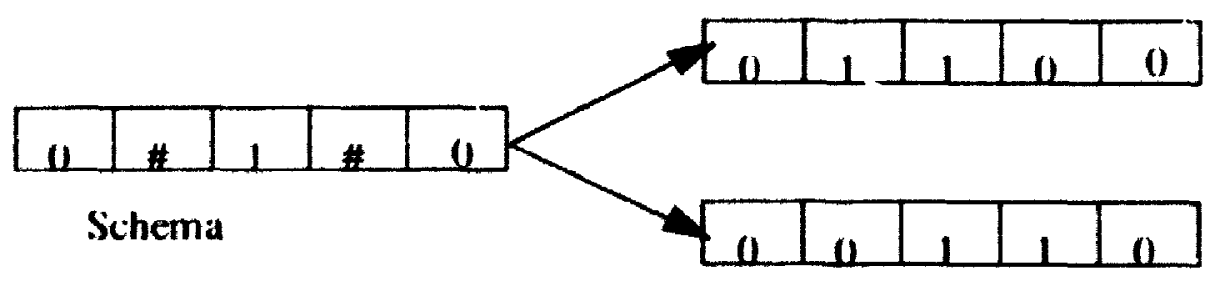

\section{Figure I Examples of schema}

The first important question to answer when using genetic algorithms is how do I encode the important parameters in my optimization problem? The degree of success in using genetic algorilhms is strongly linked to solving the representation problem well. For example. choosing a representation where the genes describing a particular feature are not contiguous in the chromosome generally leads to poor genetic algorithms performance.

The next problem to resolve when encoding a problem in genetic algorithms form is to provide a firmess function. The fitness function takes the chromosome and generates a single value which represents the utility of a particular solution. The fitness function is analogous to the objective function found in Operational Research. In some domains such as function optimization, the fitness function is obvious - it is the function itself. In others. such as the partitioning of $\mathbf{m}$ objects into $\mathrm{k}$ classes, the fitness function is less obvious and requires considerable thought. The ideal fitness function should, as far as is possible, provide an accurate mapping between the utility of the solution in the real world and the modelled world such that the relative fitness values of potential solutions are accurately captured. As an example of a poor fitness function consider a function in which the fitness of solution $A$ is less than the fitness of solution $B$ while the real world utility of solution $A$ is greater than that of solution B. Such a fitness function could never hope to find an aceptable optimal solution. This is discussed in a later section. 
Having resolved the representation and utiity mapping problems. all that retmaims is the mechanics of the genetic algorithm itself. The genetic al gorithm can lest le dexwilked by the program below:

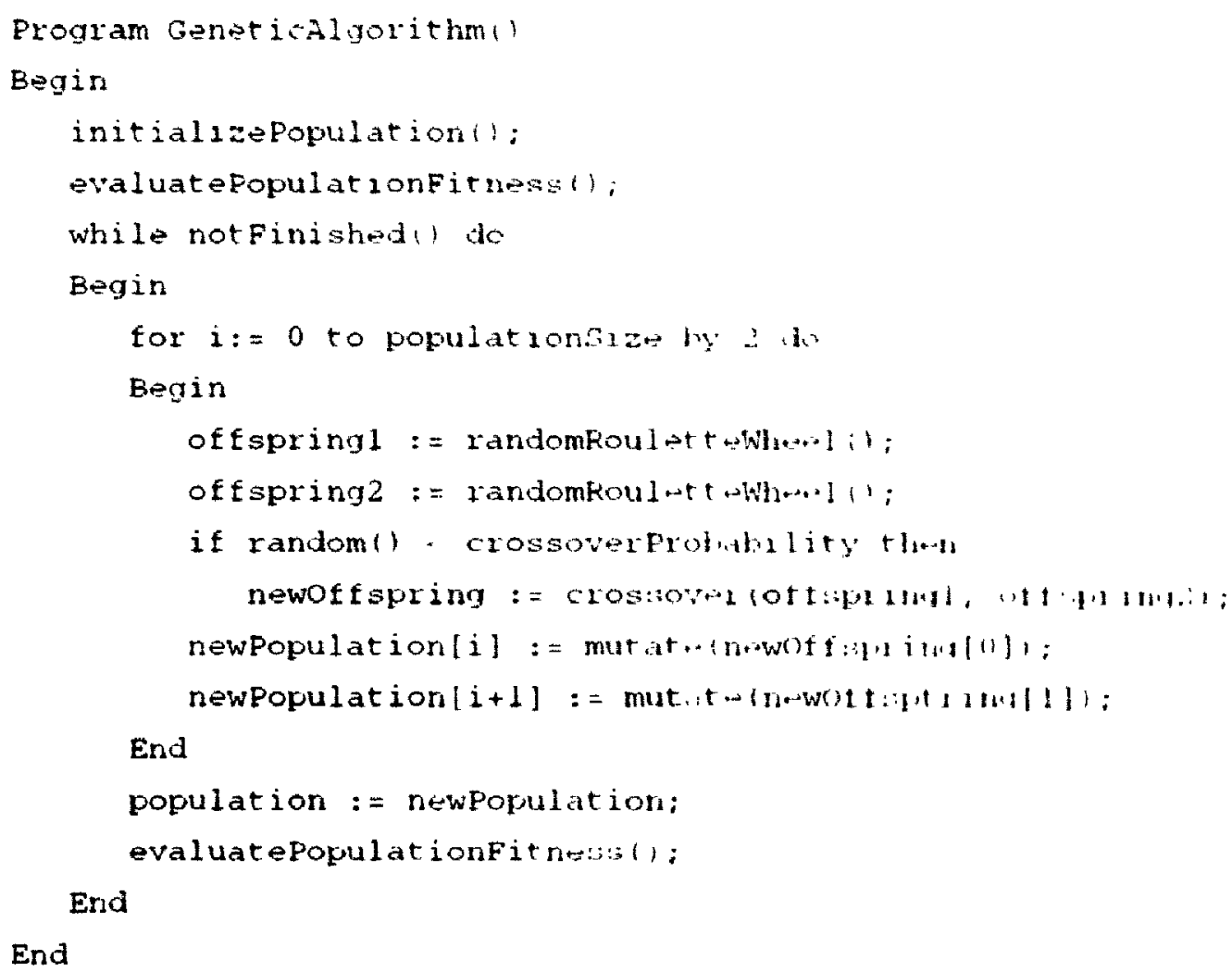

The various procedural elements of the program are descrilked in the following: sections.

\subsection{1 initializePcpulation}

The initializePopulation procedure is quite simple; we crealc a population of chromosomes where each chromosome is a binary vector of $I$ bils. All $/$ bils for each chromosome are initial ized randomly.

Program control parameters are: lengthOfC hromusome and populationsizer. 


\subsection{2 notfinished}

The notfinished function returns true if the number of trials does not exceed a program threshold, and the fitness of the best individual is less than a program threshold.

Program control parameters are: maxTrials and fitnessThreshold.

\subsection{3 evaluatePopulationfitiness}

The evaluatePopulationfitness procedure uses the fitness function referred to above in order to compute the fitness of each chromosome in the population. These val ues are stored as part of the population.

\subsection{4 random}

The random function returns a number in the range $(0,1)$.

\subsection{5 randomRouletteWheel}

The randomRouletteWheel function is used to choose which chromosomes from one generation should be reproduced in the next one. This is done by calculating an imaginary roulette wheel where each chromosome has a probability of selection calculated from:

$$
p(1)=\frac{f(i)}{i_{\text {atp }}}, f_{\text {ang }}=\sum_{t=1}^{\text {mpulaumsue }} f(i) / \text { populationSize }
$$


This is called fitness proportional selection. Viewed as a roulctie whed. for a population of five chromosomes. Figure 2 might be visualized.

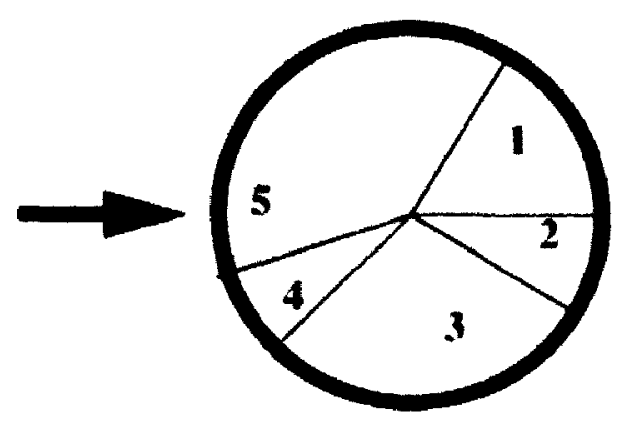

\section{Figure 2 Population Roulette Wheet}

In order to generate the new population. the imaginary roulette whect is ypun a total of populationsize times and each time wherever it stops. that chromosome is re, produced in the new population. Referring to Figure 2. chromosome 5 should the reproduced in the next generation. In fact. Lhromosome 5 cccupies approximatcly $41 \%$ of the roulette wheel and we would expect in the neighborhosd of that persentage of the new population to be chromosome 5. Obviously, several chromosomes will appean multiple times in the new population and it is just this mechanism - survival of the fillest that causes highly fit members of the population to dominate subsequent genterations.

\subsection{6 crossover}

The crossover procedure takes two chromosomes as input and generates two $11 \cdot \mathrm{w}$ chromosomes as output. The crossover operation occurs by exlecting a position between 1 and $1-1$ in the first string. This identifies two substrings $A_{1}$ and $\Lambda_{2}$. where $\Lambda_{1} A_{2}$ is the same as the original string $A$. The same position is used for string $B$, generating lle 
substrings $B_{1}$ and $B_{2}$. The crossover procedure returns the strings $A_{1} B_{2}$ and $B_{1} A_{2}$. Ciraphically, this procedure is shown in Figure 3:

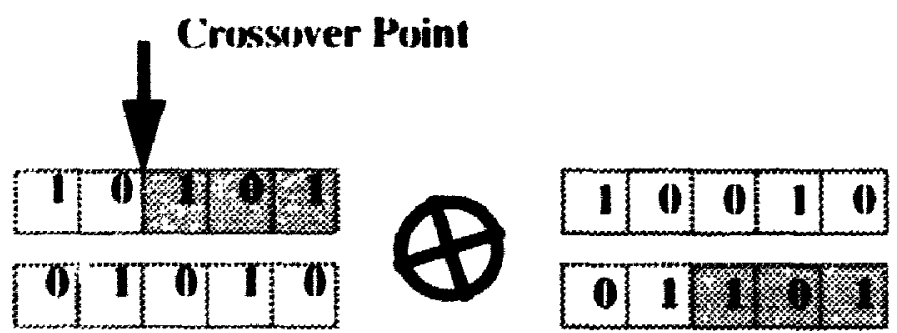

Figure 3 Crrossover in action

In the above figure, 17 is meant to represent the crossover operator. As can be seen from the above example, completely different chrornosomes can be returned as a result of the action of this procedure. It should also be noted that the crossover procedure occurs under the control of a program parameter - crossoverProbability.

\subsection{7 mutate}

The mutate procedure allows modified alleles is be introduced to the new population after crossover. The mutate procedure takes a chromosome and for coll h bit executes the following:

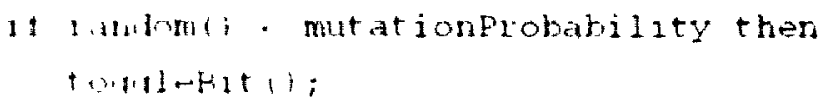

The mutation of an input chromosome occurs under the control of a program parameters - mutatiomProbability. The mutation operation ensures that the population maintains some diversity as we proceed from generation to generation.

\subsubsection{Summary}

As can the seen from the above, genetic algorithms are simple procedures which reply on a survival of the fittest mechanism drawn from the Darwinian view of 
evolution. The procedure can be seen 10 be evolutionary as clements of eath chromosome are exchanged and carried forward into the nevt generation - this being the purpose of the crossover operator. The crossiner operator works by identifying building blocks which are exchanged and that these building blocks lend w grow as we close in on a global optimun. Now, having seen the waly the crossoncer operator works. it is obvious why genes of particular feature should to grouped contiguously in the chromosome.

Genetic algorithms thus posses the crystallization property described earlier. They combine elements of random search (as they can search multiple promising alreas of the solution region) with a use of current solution knowledge (through the reproxlucince mechanism). In fact, if the crossover Probability is set to zero and the mututionl'robalpility is set to a very low value, the genetic al gorithm reduces to a hill climber strategy. If, on the other hand, the mutationProhability is set to a very high value, ihe genetic al goriulum reduces to a random search strategy.

\section{$2.2 \quad$ Formalism}

A genetic algorithm can formulated as an 8 -tuple

$$
\begin{array}{cc}
G A=\left(P^{0}, \lambda, l, s, \rho, \Omega, f, t\right) & \\
P^{0}=\left(a_{1}^{0}, \ldots, a_{\lambda}^{0}\right) \in I^{\lambda} & \text { initial population } \\
\lambda \in N & \text { population size } \\
l \in N & \text { length of individual } \\
s: I^{\lambda} \rightarrow I^{\lambda} & \text { selection operator } \\
\rho: I \rightarrow \Omega & \text { operator determination function } \\
\Omega \subseteq\left\{\omega: I \times I^{\lambda} \rightarrow \Gamma \times I\right\} & \text { genetic operator set } \\
f: I \rightarrow R & \text { fitness function } \\
t: I^{\lambda} \rightarrow\{0,1\} & \text { termination criterion }
\end{array}
$$


$f^{\prime \prime}$ is the initial randomly generated population and $\lambda$ is the number of individuals representing one generation (i.e. the number of off spring produced in a single generation ). I is the length of the representation.

The selection operator $s$ produces an intermediate population $P^{p r}$ from the population $P^{\prime}$ by the generation of copies of $P^{\prime}$ according to the probability distrihution:

$$
\begin{gathered}
\Gamma \ni p_{s}: \quad I \rightarrow|0,1| \\
p_{s}\left(a_{i}^{t}\right)=f\left(a_{i}^{t}\right) / \sum_{j=1} f\left(a_{j}^{t}\right)
\end{gathered}
$$

In other words, proportional selection. This leads to the expectation of individual $a_{1}^{t}$ to occur $\eta=\lambda_{p}\left(a_{1}^{t}\right)$ times in generation $t+1$.

Afier the selection phase has taken place the available genetic operators are applied. The application of genetic operators can be thought of as a mapping $p: l$, $\Omega$ which determines an operator $\omega_{t}^{r} \in \Omega$ for each individual $a_{1}^{r t} \in P^{r t}$ which will be applied to this individual $\rho\left(a_{1}^{\prime \prime}\right)=\omega_{1}^{t} \quad \forall i \in\{1, \ldots, \lambda\}$. The operator which is applied 6) the intermediate population is generally, in fact, a composite of the crossover and mutation operators.

\subsection{Genetic Algorithm Design Issues}

There are two extrenely important influences in genetic search: population diversity and velective pressure These factors are strongly related. An increase in the selection pressure decreases the diversity of the population. and vice versa. Strong selection pressure promotes the convergence of the genetic search process: a weak selection pressure can render the search procedure ineffective. Whitley |Whitley, 1989| observes:

"it can be argued that there are only two primary factors (and perhaps only two factors) in genetic search: population diversity

1. This will be shown in Chapler 4. 
and selective pressure $|\ldots|$ ln some sense this is just anothen variation on the idea of exploration versus exploitation that has been discussed by Holland and others. Many of the varrous parameters that are used to 'tune' genetic search are really indirect means of affecting selective pressure and population diversity: A. selective pressure is increased, the search focuses on the lop individuals in the population. but because of this 'exploitation' genetic diversity is lost. Reducing the selective pressure (or using a larger population) increases exploration' hecause more genotypes and thus more schemata are involved in the search."

Thus when casting a problem in a form such that it can be solved using a ge'netic algorithm, a number of questions have to answered and design decisions makle. The'se decisions are addressed in the following sections.

\subsubsection{Representation}

Traditional genetic algorithms work with a fixed reprexentation i.c. strings of a uster defined length. All members of the population have the same length and contann the salli: features which are to be represented in the problem. To use the language of gentetics, the phenotype to genotype mapping has to be determined. The encoding essentially takes ante of two forms - binary or non-binary. In determining an encoding for a problem. the statement, "binary or non-binary" means choosing the units of genetic material which can

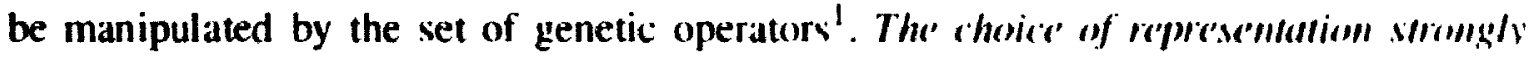

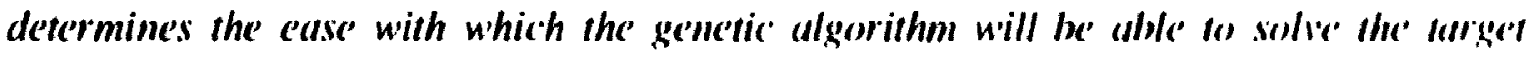
problem.

\subsubsection{Alphabet Choice}

Binary representations are often used in the solution of genctic alforithm problems. When binary representations are used, each object variable collokled in the bit string is represented using the $\{0,1\}$ alphabet. A mapping, which is usually onc 10 (nIt ${ }^{2}$ between the object variable and encoded string, is then defined.

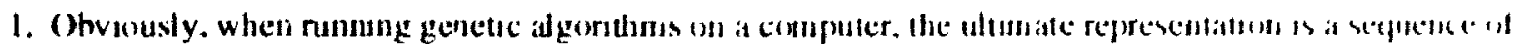
hits.

2. Unless synonyms are required. 
The $\left\{(1,1\}^{1}\right.$ alphabet is most often used because of the belief in the principle of Minimum Alphabets, which can be stated |Goldberg, 1989, page 8()]:

"The user should select the smallest alphabet that permits a natural expression of the problem."

Recently, in |Antonisse. 1989|, the principle of Minimum Alphabets has been questioned and empirical evidence for the efficient searching of spaces with higher cardinality alphabets or direct manipulation of schema can be found in [Deugo, Oppacher, 1992], $\mid$ Belew, $|y(x)|^{2}$.

Autonisse argues that:

"The more expressive alphabet is seen to carry much more power in an informationally equivalent environment. More expressive languages seem, then, to provide finer-grained tools for the construction of adaptive plans."

This work has subsequently led to an interest in more obvious encoding, such as floating point |Janikow, Michalewicz, 1991|. From their empirical studies at least, they find that:

"... the floating point representation is faster, more consistent from run to run, and provides higher precision (especially with large domains where binary encoding would require prohibitively long representation). At the same time its performance can be enhanced by special operators to achieve high (even higher than that of the hinary representation) performance accuracy."

The binary representation maps naturally to bits on a computer, but often a byte is used to represent a single allele for reasons of processing efficiency ${ }^{3}$ - bit manipulation operators are not then required. Binary alphabets allow the mutation operator to be defined very simply: in fact as the (probabilistic ${ }^{4}$ ) toggling of a single bit.

1. This alphathet is llie smallest alphathet that can te used in a genetic algorithn.

2. Dired miantpulation of selkema. al though at tractive. does come at some price in that the fitness of partial solut ims w the underly ing problem must he obtained. It is unclear whether this is an ateractive addition to Eenetle search. Sece. fir example. |Goldherg. et al. 19911 .

3. This is wh on the GENEsYs system huilu by Back.

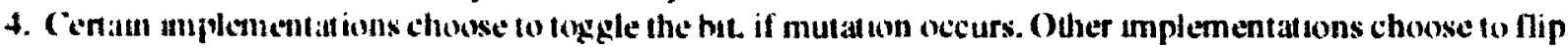
a cion in order 1 (1) dermine whether the allele will be a 1 or a 0 . The implementations are equivalent if the latler anplene'ntation has double the prohahility of mutation of the fomer. 
The importance of this mapping from problem to genetic representation camnot is overstated, as inefficient or inadequate mappings will ensure that the crossover and mutation operators will be unable to find an optimal solution. For this reason, the principle of Meaningful Building Blocks has been proposed. This can he stated as in |Cioldluerg. 1989, page $8(1)$ :

"The user should select a coding so that short low-order schemata are relevant to the underlying problem and relatively unrelated to schemata over other fixed positions."

Stated another way, related bits should be grouped cogether. This principle arises as a consequence of the design of the crossover operator. We will return to this point in a later section.

\subsubsection{Encoding Strategy}

Once an alphabet has been chosen, what remains is to map the object variahles onto the target alphabet. The simplest of all encoding strategies is the binary encoding straltegy where the ordinal value of the object variable is mapped directly onto a number of hits. This is shown schematically in Figure 4.

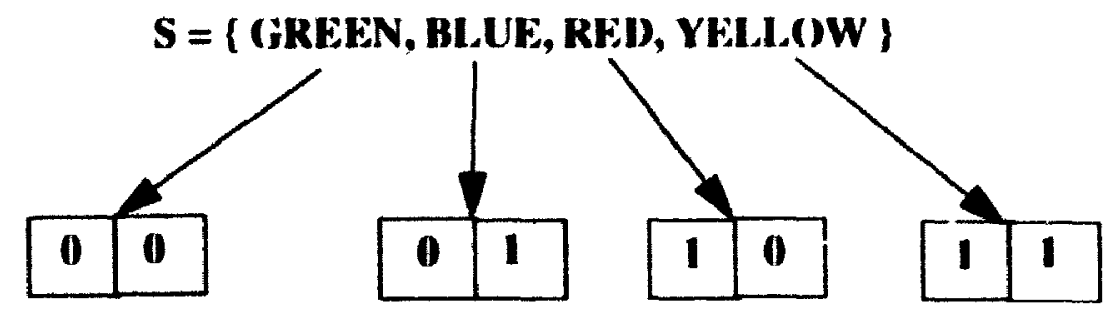

Figure 4 Binary coding example

While this works well for problems in a symbolic space, where the mappme is straightforward; in problems where floating point values are to be represented the choice is less otvious.

Studies have been performed upon the effect of representation and hidden bias in genetic algorithm encodings [Caruana, Schaffer, |9x8|. In [Caruana, Schaffer, |9xx|, results 
clearly demonstrate that Gray coding for function optimization - where real values are being manipulated - result in superior genetic algorithm performance. Figure 5 shows the above example using Gray coding.

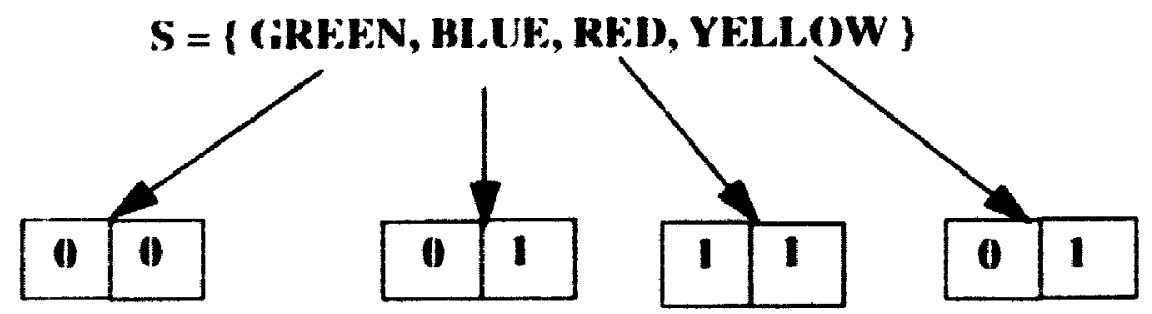

Figure 5 ( iray coding example

It can be seen from the above figure that flipping a multiple bits causes the decoded value to represent the preceding value (usually if the high bit is flipped) or the succeeding value (usually if the low bit is flipped) ${ }^{1}$. By using Gray encoding, single bit flipping (mutation) produces a value one above or one below the original value. Put another way, single bit mutations move the decoded value by the same extent regardless of the bit mutated. Table I shows the gray codes for the integers 1-15.

Table 1 Binary and Gray Codes

\begin{tabular}{|l|l|l|l|l|}
\hline Value & $\begin{array}{c}\text { Binary } \\
\text { Code }\end{array}$ & $\begin{array}{c}\text { Hamming } \\
\text { Distance }\end{array}$ & Gray Code & $\begin{array}{c}\text { Hamming } \\
\text { Distance }\end{array}$ \\
\hline 0 & 0000 & - & 0000 & - \\
\hline 1 & 0001 & 1 & 0001 & 1 \\
\hline 2 & 0010 & 2 & 0011 & 1 \\
\hline 3 & 0011 & 1 & 0010 & 1 \\
\hline 4 & 0100 & 3 & 0110 & 1 \\
\hline 5 & 0101 & 1 & 0111 & 1 \\
\hline 6 & 0110 & 2 & 0101 & 1 \\
\hline 7 & 0111 & 1 & 0100 & 1 \\
\hline
\end{tabular}

1. Assummeg a toroidal structure to the oblect values. 
Table 1 Binary and Gray Codes

\begin{tabular}{|l|l|l|l|l|}
\hline Value & $\begin{array}{c}\text { Binary } \\
\text { Code }\end{array}$ & $\begin{array}{c}\text { Hamming } \\
\text { Distance }\end{array}$ & Gray Code & $\begin{array}{c}\text { Hamming } \\
\text { Distance }\end{array}$ \\
\hline 8 & 1000 & 4 & 1100 & 1 \\
\hline 9 & 1001 & 1 & 1101 & 1 \\
\hline 10 & 1010 & 2 & 1111 & 1 \\
\hline 11 & 1011 & 1 & 1110 & 1 \\
\hline 12 & 1100 & 3 & 1010 & 1 \\
\hline 13 & 1101 & 1 & 1011 & 1 \\
\hline 14 & 1110 & 2 & 1001 & 1 \\
\hline 15 & 1111 & 1 & 1000 & 1 \\
\hline
\end{tabular}

The binary encoding can make search difficult for genetic algorithms leccause the Hamming distance between adjacent values is not constant. Actually, it cam be as large' an the number of bits in the representation. However, the similarity exploiled by the genetic al gorithm is schema similarity; the genetic algorithm's recombination operators implicitly assume that genetically similar strings have similar perfomance. It is expected that the effectiveness of the genetic al gorithm will improve when the Hamming distance betweell encoded values has a similar furctional form to that of the distance between values in the problem domain. This is certainly the case in Table 1.

For this reason - the lack of a Hamming cliff - Gray coling is generally preferred over Binary crding in function optimization.

\subsubsection{Reproductive Scheme}

A reproductive scheme consists of two components: the sampling incthod and whe ther the whole population or some subset of the population is replaced during a single generation.

When the whole population is replaced during a generation this is called a generatimal scheme. In a generational scheme, the set of parents is fixed until $n$ off spring have Ixe'tI reproduced from that set of parents and then the parents are discarded. Parents, therefore. live for a single generation. When a subset of the population is replaced in any given 
generation, the scheme is called steady state. In a steady state scheme, only a few members of the population are changed and thus parents can live for many generations hefore dying.

Steady state whemes are now receiving more attention in the genetic aigorithm literature as they are thought to have advantages such that (a) schema fitness versus percentage in the population works out properly as the fixed point of the system, and (b) good members of the population float to the top of the list ${ }^{1}$ where they are protected from deletion, and that (c) poor members of the population sink to the bottom where are more likely to be deleted.

Various reproductive schemes, or sampling mechanisms, have been investigated for genetic al gorithms in order to improve upon the basic roulette wheel scheme. The roulette wheel, or fitness proportional reproductive scheme has the disadvantage that highly fit members of the population can quickly dominate. or crowd out, weak members of the population leading to premature convergence.

Although a partial solution is to allow raw fitness to be scaled - see section 2.3.3.1 - the choice of sialing mechanism and sealing coefficients implies that new genetic algorithm parameters need to be chosen that are certainly problem specific. Reproductive schemes, on the other hand, offer a more robust mechanism for the prevention of premature convergence.

In the earliest study [DeJong. 1975], seveid variations on the basic scheme were investigated. The first variation - the elitist model - enforced the preservation of the best individual in the population. The second variation - the expected value model - reduces errors due to poor sampling. In this variation, the number of offspring generated in the next generation is computed using:

$$
n(i)=f(i) / f_{a v g} \quad f_{\text {arg }}=\sum_{i=1}^{p o p u l a t i o n S i z e} f(i) / \text { populationSize }
$$

1. assumm somk forn of pupulation ranking. 
where $n(i)$ represents the expected number of coptes of an individual that are 10 Is reproduced in the next generation. As offspring are reproxluced using the standard roulctte wheel, the $n(i)$ is reduced by 0.5 or 1 . When $n(i)$ falls below zero, that individual is not a candidate for further selection.

In the elitist expected model. the best chromosome is guaranted to be reproduced. while all other selections are performed using the expected model algorithm. In the crowding factor model, a newly generated individual replaces one from the parent gencration - the parent being selected based upon its similarity to the offspring. In this sehente, a hamming distance measure is typically used in order to determine similarity. The crowding factor determines the set of individuals which are considered to be similar to the offispring and are therefore candidates for replacement. Replacement of an individual from this $x+1$ is done randomly, or using the proportional fitness mechanism.

Brindle |Brindle, 1981| proposed other alternatives for selection schemes: deterministic sampling, remainder stochastic sampling without replacement, stochastic tournank-nt. remainder stochastic sampling with replacement, and stochastic tournament. Bimpirical studies showed improvement over the basic fitness proportional mechanism, and in particular, the remainder stochastic sampling with replacement method was shown to have superior properties. This scheme uses the following:

$$
n(i)=f l o o r\left(f(i) / f_{\text {avg }}\right) \quad f_{\text {avg }}=\sum_{i=1}^{\text {populationSize }} f(i) / \text { pepulationsiziz" }
$$

In this scheme, a certain number of individuals are guaranteed in the mext gencration as given by n(i). The remaining offspring compete, using a rouletle whecl mechamism. according to the fractional value, $n(i)-f(i) / f$ arg. The remainder whotastuc sampling with replacement method is arguably the most popular sampling method uxed by genetic al gorithm practitioners today.

Selection methods ba:ed upon ranking. rather than fitness, have al so been studied llsaker, 1985|. |Whitley, 1989a). Baker, in particular, uses an adaptive mechanism in order to 
control premature convergence. This is described at length in Chapter 6, section 6.1.1 al ong with other mechanism for the avoidance of premature population convergence.

Unfortunately, ranking selection schemes violate the Schema Theorem ${ }^{1}$ and so theoretical support for their optimal allocation of trials does not exist. However, the aforementioned studies have indicated that they prevent scaling problems (see section 2.3.3) and provide better control over the selection pressure implicit in the genetic al gorithm.

A comprehensive review of sampling mechanisms, and thear complexities, can be found in |Baker. 1987| and a taxonomy of selection schemes can be found in |Bäck. Hoffmeister, |थ)ा!.

\subsubsection{Fitness Function}

The fitness function in a genetic algorithm represents the abstraction of the real world objective function. The choice of fitness function is the single most important design decision made in a genetic algorithm. Poor choice of fitness function will ensure poor genetic algorithm results. If the fitness function does not map to the real world objective function such that equivalent trends are seen in each function, the genetic algorithm will be unlikely to find a global optimum easily. The fitness function is actually an abstraction of the environment of the problem to be solved.

Oiten, the objective function cas. span a wide range of values, both negative and positive. As a result, it is consequently necessary to map the underlying natural objective function ic a fitness form through one or more mappings. For example, given an objective function $g(x)$, which is a cost function that we wish to minimize, a useful fitness function might be:

$$
\begin{gathered}
f(x)=k_{\max }-g(x) \quad g(x)<k_{\max } \\
f(x)=0 \quad \text { otherwise }
\end{gathered}
$$

1. Sect Chapter 4. 
Alternatively, when trying to maximize a function which can take both negative and positive values, an appropriate fitness function might he:

$$
\begin{array}{cc}
f(x)=k_{\text {min }}+h(x) & h(x)+k_{\text {min }}>0 \\
f(x)=0 & \text { otherwise }
\end{array}
$$

The fitness function. then, should the as direct a mapping as possibls firm the objective function. However. the fitness function must also lake into account llic properties of the selection algorithm as with proportional selection it is possible for one or two highly fit members of the population 11 dominatte during reproduction and thereby cause other, less fit, members not to be reprofucied at all. As a consequence, certain bit patterns get lost and the population can converge too quickly before the genetic algorithm has had the opportumity w explore a reasonable proportion of the search space.

For this reason. two modifications to the basic genetic algorithm have heen proposed. Firstly, fitness scaling is possible. This is described in the next section. Scomully, reproduction strategies other than those based upon relative fitness values have Ixe'l investigated. This was previously described in section 2.3.2.

In more recent work on genetic algorithms, reproductive strategices other that! fitness proportional selection have tended to be preferred over fitmess sualing muchamisms as a means of controlling population diversity loss.

\subsubsection{Scalling}

Scaling of the fitness function is undertaken in order to regulale the numlxer of copies of any one string that will be reproduced to the mext genteration. If letl fu, normal roulette wheel selection, extraordinary individuals could casily take over a very large proportion of the population in a single generation. This is highty undesirable as it causes premature convergence. Empirical xaling studies were first performed by Bagley |Bagley, 1967|, and Koxenberg |Rosenberg. 19,7|. Scaling attempts to retain diversity in the population but still provicke sufticient 
evolution pressure such that significant numbers of highly fit individuals will be reprosluced in a subsequent generation once they emerge during the course of a genetic algorithm run.

Several scaling procedures have been studied. Linear scaling takes the unscaled, or raw fitness, and generates a fitness defined by:

$$
f=A \dot{f}+B
$$

where $A$ and $B$ are scaling coefficients and $\dot{f}$ is the raw fitness. Typically, $A$ and $B$ are chosen such that: $f_{a v g}=\dot{f}_{a v g}$ in order that the average members of the population contribute one member, on average, to the next generation. Similarly, the maximum fitness can be constrained, $f_{\max }=\tilde{f}_{\max } C$, where $\mathrm{C}$ is the expected number of copies of the fittest members in the next generation. Typically, $\mathrm{C}$ lies between 1.2 and 2 .

This form of linear scaling has the effect of ensuring that superfit members of the population do not generate a large number of copies of themsrlves in the next generation. Although linear scaling works well, it can generate negative fitness values ${ }^{1}$. This occurs when the population contains mostly highly fit members but a few inferior members remain.

Forrest |Forrest, 1985| proposes the use of population variance in pre-scaling raw fitness values:

$$
f=\dot{f}-\left(f_{\text {arg }}-c \cdot \sigma\right)
$$

where $c$ is a constant hetween 1 and 3 and negative fitness values are truncated to zero. Fitness scaling can then cxcur without danger of negative results.

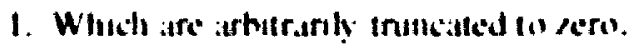


Power scaling inas also been suggested |Ciillies, 19851. In this mallung procodure. the raw fitness is raised to some positive power. as shoun below:

$$
f=\ddot{f}^{h} \quad k>0
$$

For $k>1$, the fitness range is stretched, meaning that higher than average fitmos individuals will get substantially more than one offspring all average. For $k$ \& the fitmess range is shrunk, meaning that inferior individuals will not the clomdid out during the reproduction phase.

\subsubsection{Operators}

Operators in genetic al gorithms are used to manipulate one or more vrings in a populantum in order to produce one or more off spring. In a conventional penetic stgonthm. the crossover and mutation operators have been applied secumentially. as outlined in llic Program GeneticAlgorithm() on page 10. A wide range of problem-specific eperallers have been defined for particular problem domains. Specifically, the Travelling Sallevman Problem and problems involving permutations have necessitated ypecialised ciperators.

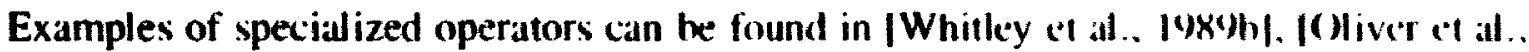

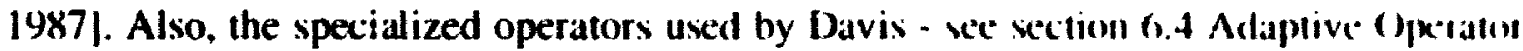
Selection on page 106 - are examples of specialized operators.

In the next three sections, three classes of operator are described. The on nperaten claswes are widely used by genetic algorithm researchers today.

\subsubsection{Crossover}

Putting aside the specialized operators alluded to above. Iwo disturct classes of comsovel operator have been described in the literature. These alc mult ponil cursoveres and uniform crossover.

Single point crossover - shown schematicaily in Figure 3 Csossover in ac tion wa page 13 is a special case of multi-point crossover. In multi-point crossover, with the wumber it 
pomils in the crossover being $n$, $n$ points are selected in the parent string. Each crossover point, plus the two ends of the string, define $n+l$ intervals. Multi-point crossover then proceeds by allocating the first interval from parent 1 to offspring 1 , the second to oft ypring 2. the third to off spring ! and so cn. This is shown schematically in Figure 6.

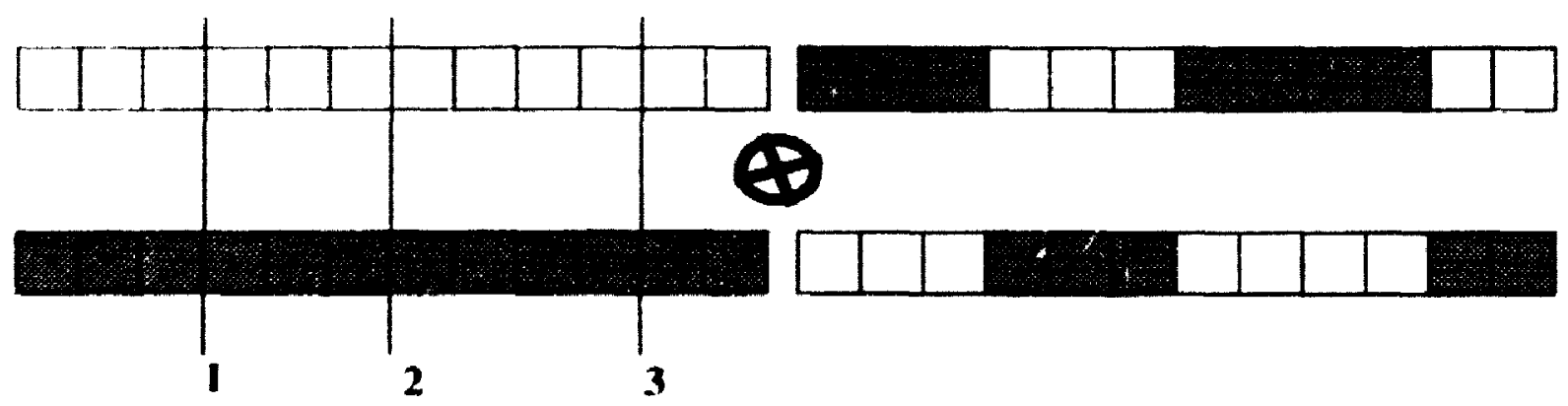

Figure 6 An example of three-point crossover

[X-Jong |DeJong. 1975| onserved that multi-point crossover operators tended to degrade oniine and offline perfurmance and he attributed this to poor survival rates of second order whemata. However, it is easier to anderstand by consideration of the number of unique operalyrs which are potentially involved in genetic search. With $\mathbf{n}$-point crossover there are $\left(\begin{array}{l}l \\ n\end{array}\right)$ different ways of picking the $n$ points. As a result, when $n$ is increased. each operatior is less likely to be picked during a particular cross, and less structure can be preserved. With more mixing and less structure. these more complex operators become more like random shuffle and fewer important schema can be preserved. However, twopoint crossover (and $n$ for that matter) does avoid the representational bias which results in single poim crossover - which tends to avoid mixing alleles as we move from the centre to the end of the string.

This appears to be at more important effect than the previous argument, as two-point crowover has cmpirically been shown to exhibit superior performance when compared to single-point crossover in a number of environments |Eshelman, et al.. $\left.1989\right|^{1} \mid$ Syswerda.

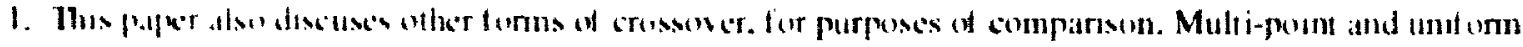

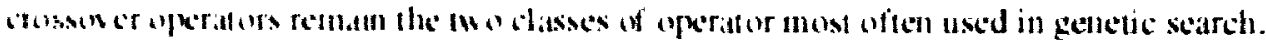


1989|. In situations where multi-point crossover is used, two-point crossover sectus fo the most favoured in the literature.

Uniform crossover due to Syswerda |Syswerda, |989)| avoids the representational hias implicit in multi-point crossover by considering each bit in turn. Thus, starting at hit I, the two parents" bits are exchanged with probability $1.5^{1}$, similarly at hit 2 and so (nn. The expected number of bits to be exchanged is thus 1/2, with I being the lellgth of the string. The uniform crossover algorithm is shown schematically in Figure 7.

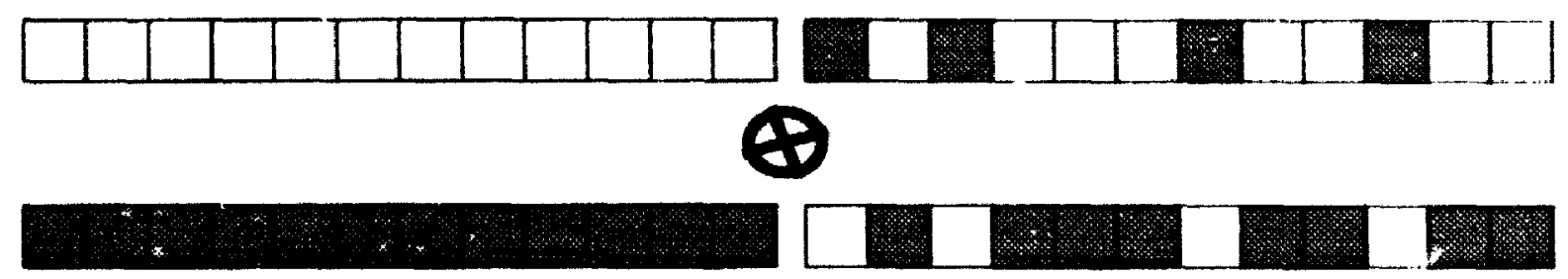

Figure 7 An example of uniform crossover operator

In [Syswerda, 1989] the unifonn crossover operator is shown to have superior performance when compared to one and two-point crossover on a wide range of problems. Syswerda argues that al though schema are being destroyed at a faster rate in the unifurm crossover operator when compared to single or two-point crossover, schema are al solecing created at a far faster rate. Based upon his empirical results, it would appear that the latle! effect dominates - although a compre!ıensive theory remains to be developed.

The improvement of the liniform crossover operator over multi-point crossover provides the motivation for its choice as principal candidate for the addition of all andaption mechanism using automata. Also, as Figure 8 clearly shows, 11.5 is not the optimal s'thng for the uniform crossover operator probability in all cases and lends support to the idea that adaption should improve the performance of the uniform operator.

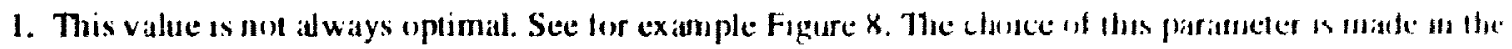

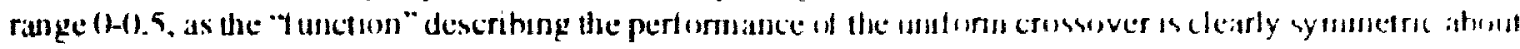
11.5. A simple symmety argument - exchangug the two parents - proves dils. 


\section{5 performance versus crossover probability}

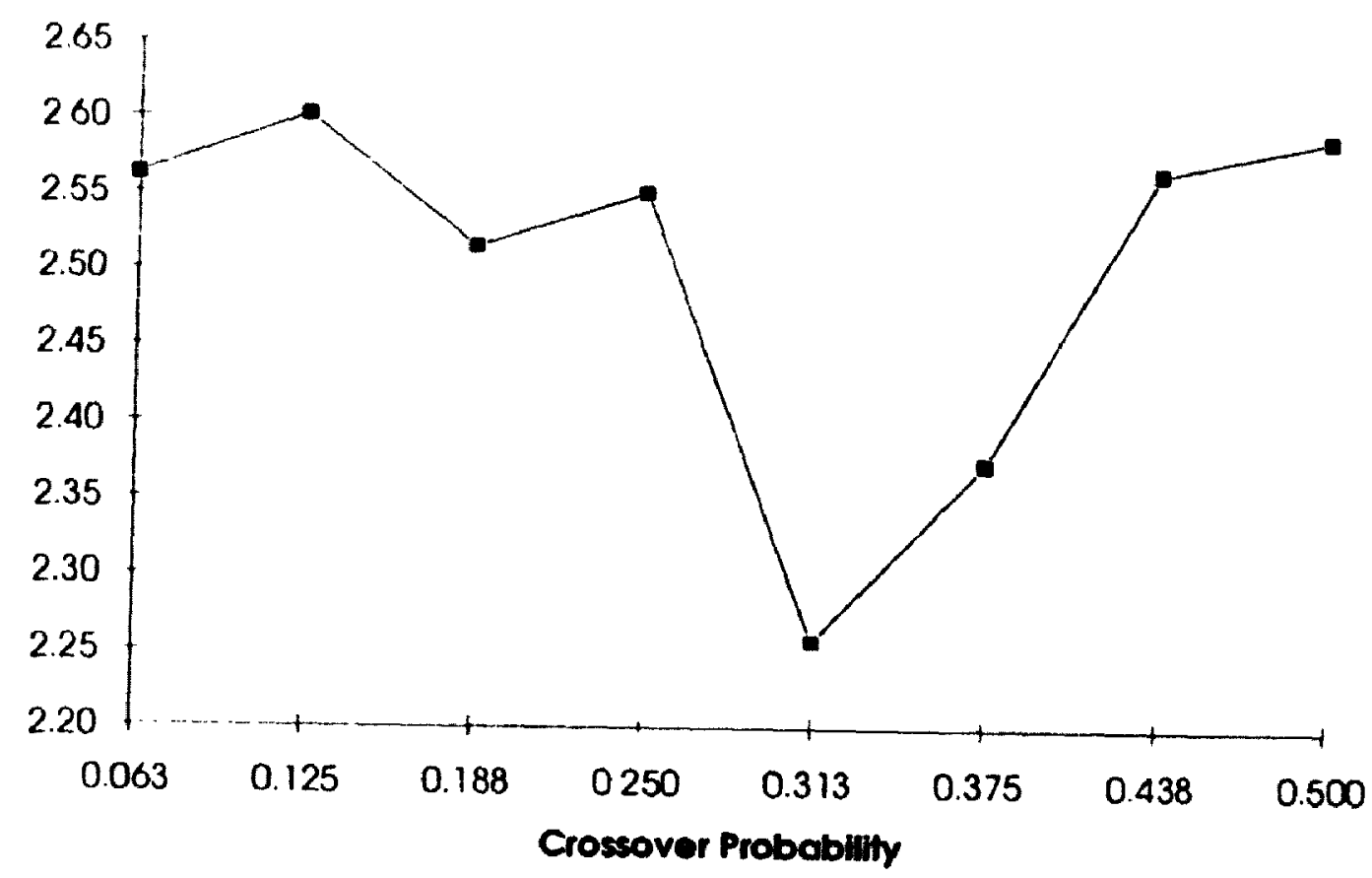

Figure $8 f_{5}$ performance for varying uniform crussuver probability

Note: the results obtained for $f_{5}$ were gathered over 100 runs of $10,0(0)$ trials using a population of $5(1$. probability of mutation 0.001 and fitness proportional reproduction. The information plotted is the best-at-trial at $10.1(1)$ trials.

\subsubsection{Mutation}

Mutation is often considered to be a "secondary" operator by genetic algorithm practitioners and is often ignored by the genetic programming community. However, Bäck and others from the Evolutionary Strategy community have shown that complex problems call be solved using just mutation. Similarly, Ray [Ray. 1991/ has shown that complex behaviour can result from the action of the mutation operator alone without the need for sexual reproduction'. The mutation operator works on a single string. The role of mutation

I. 1.e. crosselfet 
in genetic search is two-fold. Firstly. mutation provides a local search capability is it makes small changes in the structure of the offspring. Also. the diversity of the population is increased by the action of the mutation operator.

Research have been undertaken to make the mutation operator depend upon the diversity of the population and this is the subject of section 6.1.2 Diversity Maintenance using Entropy on page 99.

In its simplest form, the mutation operator toggles bits as it moves along the hit string. However, in higher cardinality alphabets the mutation operator requires more specification in that a mapping is required for each possible value. For example, in the situation where floating point values are being mutated, a mutation increment is usually defined and cither added to, or subtracted from, the current value. In |Ray, |Y9||, mutation takes the form of a counter being incremented by two or zero when one was indicated, or a copy insiruction was incorrectly interpreted along with the more conventional bit flipping.

\subsubsection{Inversion}

The inversion operator was originally proposed by Holland |l Iolland, 1975 | as a means by which an initially poor problem representation could be improved through genetic search. The inversion operator works on a single string. Under inversion, two points are chorell at random along the length of the string and the string is cut at those points. The conds arce then exchanged. This operation is shown schematically in Figure 9.

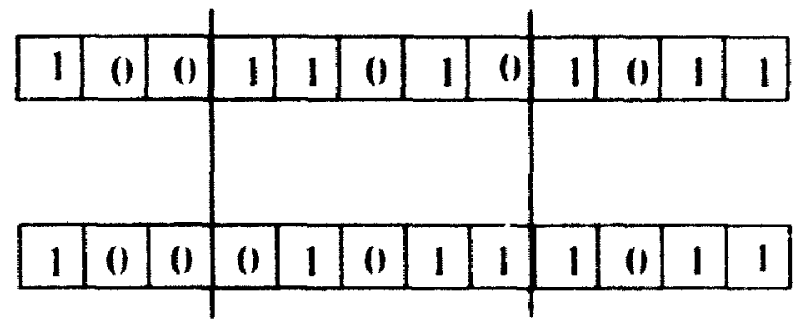

Figure 9 An example of the inversion operator 
It a not clear how the inversion operator is helping in the search for a new representation it looks as though bits are just being shuffled about. In fact. Bagley [Bagley, 1967 pg. 168] states:

"The inversion results ... were somewhat |sic| disappointing. The most obvious effect of inversion is the large increase in the length of the runs. Recall that one of the consequences of inversion is a decrease in the effective crossover rate since crossover is inhibited hetween parts of the chromosomes which are not locus homologous. As we have seen, a lower crossover rate has an adverse effect of the simulation and it is this effect that was predominant in our results. The desirable consequence of inversion. which is the appearance in the population of gametes whose geographical gene linkages reflect combinations ... was not observed."

Although Cavicchio used an inversion operator in a pattern recugnition detector design task |Cavicichio, $|970|$, very few genetic algorithm implementations tend to use the operator today. It is believed that the more powerful two-point and uniform crossover operators, along with improved population reproduction strategies, have rendered the operator obsolete.

\subsubsection{Parameter Settings}

The genctic algorithm requires the setting of a number of parameters. These are the probability of mutation the probability of crossover, and the population size. While the values presented her. w. a cast in stone" they provide a reasonable starting point for investigations ir : viving genetic algorithms.

For functions in the test bed, from $f_{1}$ to $f_{10}$. |Schaffer, et al., 1989| reasonable performance has been obtained using:

Table 2 (ienetic algoritt a parameter setting defaults

\begin{tabular}{|l|l|l|}
\hline \multicolumn{1}{|c|}{ Variable } & \multicolumn{1}{c|}{ Meaning } & \multicolumn{1}{c|}{ Value } \\
\hline$\rho_{m}$ & Probability of mutation & $0.005-0.01$ \\
\hline$\rho_{c}$ & Probability of crossover & $0.75-0.95$ \\
\hline$N$ & Population size & $20-30$ \\
\hline
\end{tabular}


For function optimization at least. it seems that Gray coding should le used in conjunction with the remainder stochastic sampling with replacement method for population reproduction in a generational genetic algorithm. A uniform crossover operator we'ms 10 show most promise for high performance across a wide range of problems. 


\section{Overview of Genetic Algorithms}

\section{$3.1 \quad$ Overview}

This chapter consists of two distinct sections. First, the historical and biological motivations for genetic algorithms are discussed. Second, a review of the applications of genetic al gorithms is presented. The principal purpose of this chapter is to highlight the diversity of applications which have chosen to employ genetic al gorithms and to detail the design decisions that have been made during their use in the chosen problem domain.

\subsection{Motivations}

Machine Learning can be viewed as a problem of searching in some abstract solution space |Mitchell, 1982) where learning occurs through the process of identification of Inetter and better solutions.

It is particularly interesting to note that both of the most promising approaches to the design of algorithms for Maxhine Learning have as their metaphor naturally occurring systems. Connectionist algorithms draw for their inspiration on the neural structure of the brain and attempt to emulate how neurons in biological systems allow organisms to adapt to their environment. Amongst the most prominent connectionist xicentists would be Hopfield. Kohonen and Grossberg. Similarly, models of simulated evolution are altempts to mimic the way in which species become adapted to their respective environmental niches. The question answered by studying simulated evolution can be stated as. "How does evolution produce increasingly fit organisms in environments which are highly uncertain for individual organisms?" Amongst the most notable 
scientists attempting to answer this question are Holland. Maynard Simith and mors recently Ray.

It is generally believed that complex life systems have evolved via Darwinian evolution where the principal force behind evolutionary change is selective pressure applied by an ever-changing environment. Darwin's seminal work. "The Origin of Species" II );rwill. 1859] not only represented a revolution in scientific thinking but also the Iriumph of scientific method over religious doctrine. It is important to note that it appears that there can be intelligent behaviour without a designer.

The above observation tends to support the view that symbolic approaches fo learning tend to be simulating learning at the meme ${ }^{2}$ |Dawkins, $1986 \mid$ level - or rather cultural learning - where knowledge is passed from one generation to the llext in used to improve Society in the next generation. For example, the knowledge embodied in an Explanation Based Learning system forming the Domain theory could be said (o) consist of menk' which have been derived from a previous system and passed from compuler to compuler or brain to computer.

It has also been noted that evolution has occurred in a hierarchicai fashion with less complex organisms, or structures, forming the basis for increasingly $\because$ mplex syskms. Our own brain is a remarkable example of such an evolutionary process. The brain is the result of thousands, perhaps millions, of years of evolution. Simple vertebrakes hill anly a a simple nervous tube, or spinal cord. This was sufficient for survival. That tulde evolved to receive sensory information through various nerve fibres and to send motor signals to contract the animal's primitive muscles. Soon it became apparent that it is easicer to move in a single direction rather than many cirections and, as such. it was more important to know what is happening in the direction of movement than ally other. $A$ s a result the frontal part of the spinal cord which was receiving signals from the preferred direction of movement became more important that other signals. This portion of the spinal cord its sensory capabilities and the brainstem - the precursor of the human brain - canle into

1. If one is to assume no divine guidance in the evolutustary process.

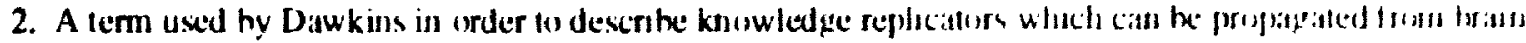
to brain. compuler to computer. or brain to computer in exiunples. 
being. New challenges from an ever-changing environment induced further development in the motor and sensory systems. The brainstem grew in order that functionality could be controlled in a better, more direct, way. The frontal part of the brainstem was enlarged by the addition of two cerebral hemispheres. The back (or posterior) part of the brain evolved into the cerebellum. The cerebellum acquired the abilities of data analysis and decision making. In short, the relatively simple nerve tube evolved into the seat of human intelligence.

The importance of this extended example is to demonstrate the following points. Firstly, systems of ever-increasing sophistication and complexity have evolved. Building hlocks are designed and are used as subsystems in higher order systems. Secondly, evolution occurs on many levels simultaneously. As higher order systems appear, the building blocks do not remain "frozen" but can continue to evolve in their own right.

Genetic algorithms offer an entirely different approach to Machine Learning when compared to symbolic learning. If the problem of feature expression is set aside for the moment (as this is not understood in Nature) genetic algorithms can be seen as a class of Machine Learning algorithm in which no "designer" is needed, but where intelligent behaviour can be observed.

The field of genetic algorithms - an abstract form of simulated evolution - is generally accredited to John Holland. although its beginnings can be traced back to biological simulations of the early 1950's. In |Holland, 1975], the Darwinian notions of evolution via a survival of the fittest mechanism are integrated with modern notions of Genetics, in a mathematical framework, in order to provide a robust search algorithm that is now called the genetic algorithm. Excellent texts describing genetic algorithms in depth can be found in [Goldberg. 1989|. [Davis, 1987, 1991] and [Michalewicz. 1992]. It was Holland's observation that adaptive processes are fundamentally optimization processes but that unitied study had previously not been possible owing to the complexity of the structures txing modified and the uncertainty of their performance in the chosen problem domain. Moreover, that an efficient adaptive process must exploit elements of its past experience in order to continue to improve. 
Holland also observed the failures of the symbolic approaches to Machine Learning. In [Michal ski et al. 1986], he observed:

"The systems lexpert systems| are brittle in the sense that they respond appropriately only in narrow domains and require substantial human intervention to compensate for even slight shifts in domain."

One of Holland's principal goals in designing improved algorithms for Mikhine Learning was that the resulting algorithms be robust and be able lo parfom in misy environments. He observed the importance of building solutions /merarc/malls - stanting: with small fragments and combining them in ever nore complex palterths. This was considered crucial to the success of genetic algorithms as the theory in rhapter 4 proves

A parallel development, called evolution strategies. (an the tracied back to experimkelts undertaken at the Technical University of Berlin in 1964 |Rechenlxerg. 197.31 and formalized by Schwefel |Schwefel, 1975|. Evolutionary strategies first saw applicalion in the area of Numerical or function optimization and were applied to funclions of continuous variables. In this, the "European" approach. less emphasis has Ineen plinced upon the biological metaphor - although the term biological is found in the title of the original 1964 paper - while in the "American" approach the terminology used in "ellotic algorithms still reflects its natural origins. Concerns continue to be expressed in the literature whenever an "unnatural" operator, or contrived selection texhnique are propomerl |Goldberg, 1989 pgs 153-154]. The biological metaphor is considered to to* valy important when talking about genetic al gorithms.

Fortunately, with increasing communication between revearchers in the evolutionaly systems and genetic algorithm camps and the coordination of conterences in Lurope and North America, an attempt to provide a taxonomy of algorithms has becn altempted. In [Bäck et al, 1991], definitions and terms are introduced which show that a getletic algorithm can be broadly classified in terms of evolutionary strategics. Essemtially. Hic genetic algorithm can be seen as being an evolutionary strategy in which (a) a unifurm representation is used for all features of the problem and (b) applies its operaturs in an unchanging way during the course of the algorithm run. Chapter 5 descrite.s the 
vimilarities and differences between evolutionary strategies and genetic algorithms in considerably more detail. It is introduced here in order to provide an historical perspective on the genetic algorithm.

In the next section applications of genetic algorithms are presented. The spectrum of problem classes that have seen the application of genetic algorithms is considerable and underscores the claim as to the power of this class of al gorithm.

\subsection{Applications of Genetic Algorithms}

(ienctic algorithms have been used in a very wide range of problem solving situations. The four international conferences on genetic algorithms have seen a wide range of application areas using genetic algorithms and it is not possible to describe those application areas here. The application areas described here are:

1. tame playing

2. function optimization

3. neural network optimization

4. design

5. automatic programming

Although this does not represent a comprehensive view of the appiication areas that have chosen to adopt genetic algorithms as a solution technique, it does indicate the diverse nature of the application areas themselves. Selected applications from these areas are described in the next five sections.

\subsubsection{Game Playing}

The Arificial Intelligence community has long considered game playing as a fertile test bed for the application of Machine Learning techniques and it is no surprise that genetic al gorithms have been used here. Essentially, the aim is to develop better and better strategies as a result of learning from experience.

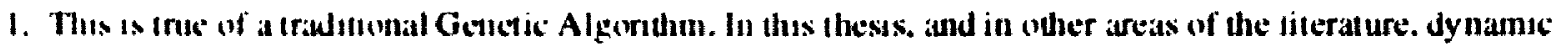

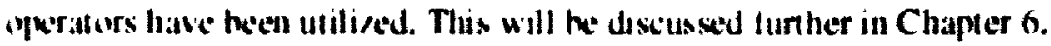


The term "genetic algorithm" appeared long hefore its association with Hollam in 1975. Bagley's work |Bagley. 1967) consisted of devising a test hed of gatlme-playing tasks modelled after the game of hexapaun. Hexapawn is played on a 3,3 chesshoard where each player starts with three pawns and tries to reach the wher side of the hatril. Bagiey chose to vary the abilities of a player by allowing search w a given munlwer of moves. He called this the "task depth".

Bagley devised genetic algorithms to search for parameter sets in game evaluation functions; comparing them to correlation algorithms'. Correlation al gorithms werce found to require a strong match between the nonlinearity of the game and the numlimearity of the correlation algorithm. However, Bagley's genetic al gorithm semed much less sensilive lu, game nonlinearity and performed well over a wide range of task depths.

The genetic algorithm proposed by Bugley strongly resembles the Ilollima genetic algorithm in that reproductive. crossover and mutation operators atre constructed. However, rather than the monoploid represintation favoured in Holland genetic algorithms, a diploid representation was used in order (1) encinle the concept of dominance. Bagley also used a non-bmary encioling fior liss problems; something which tends not to be done in moxkern genetic algorithms.

Bagley's work is doubly relevant to this thesis in that he observed the llecel for "self contained controls" and was conscious of the problems of fitness valing. He believed that at the stan of a genetic algorithm run fimess should $\mid x \cdot$ xalcel III order to ensure that no single individual be allowed to dominate the population. However, later in the run fitness should be scaled in oriler to take advantage ol small differences in the fitness values. He also suggested encoxling the prohability of mutation and crossover in the chromosome themselves. Ilowever, his lloesis dicl nol present results of suggested experiments.

The prisoner's dilemma is a classic problem of conflict and comperation. In this game, two players have a choice of cooperating with the other or defecting.

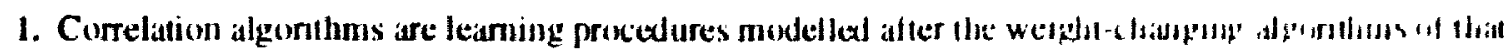

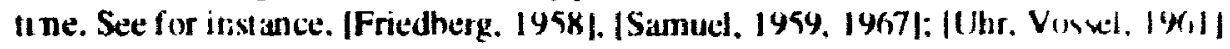


Irpending on the two players decisions, each receives a payoff according to a payoff matrix such as shown in Table 3.

Table 3 Payoff matrix for the Prisoner 's Dilemma

\begin{tabular}{|l|l|l|}
\hline \multicolumn{1}{|c|}{ Decision } & \multicolumn{1}{|c|}{ Couperate } & \multicolumn{1}{c|}{ Defect } \\
\hline Cooperate & $(\mathrm{R}=6, \mathrm{R}=6)$ & $(\mathrm{S}=0, \mathrm{~T}=10)$ \\
\hline Defect & $(\mathrm{T}=10, \mathrm{~S}=0)$ & $(\mathrm{P}=2, \mathrm{P}=2)$ \\
\hline
\end{tabular}

In the above table, $R$ represents reward, $S$ represents sucker, $T$ represents temptation and $P$ represents penalty. The object of the game is to maximize the reward received by the player over a series of rounds. As can be seen in the above table, the maximum, single round reward is received in the situation where one player cooperates and the other defects.

The first genetic algorithm solution to the Prisoners" dilemma was due to Axelrod |Axelrod. 1985. 1987|. Axelrod's idea was to have the genetic algorithm search for improved strategies capable of playing the iterated fom of the game. In order to do this, Axelrod allowed decision rules to be based upon the behaviour of the other player in the previous three moves. In order to code a particular strategy, Axelrod coded the sequence of actions as a three letter string. Thus PPP represents the situation where both prisoners defect on the three moves while TTP represents the scenario in which one prisoner defects twice, and then cooperates while the other prisoner cooperates on all three moves. A possible 64 altematives exist.

Actually. the problem is more complex in that behaviour is unknown at the hegimning of the game. Axelrod added a further six bits in order to capture the pregame assumptions of behaviour. Pregame behaviour, as Axelrod showed. was extremely important in determining the efficacy of the chosen strategy.

Strategies were evolved by having a population of candidate strategies play against a set of eight opponents taken from his computer tournaments. The success of a particular strategy was measured over a game of 151 moves and the point wore al the end of the game used as a measure of the value of the strategy. In performing 
this experiment using randomly generated initial strategies. Avelrot obtained strultegies

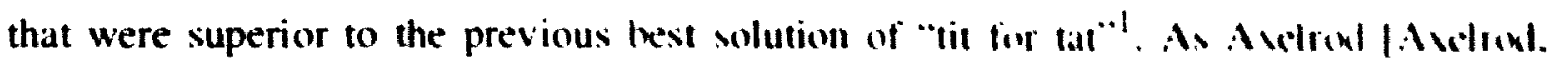
1985] observed:

"This is a remarkable achievement beciase to $\mid x \cdot$ able 10 get this added effectiveness, a rule must te able w do three things. First. it must be able to discriminate between one representative and another based upon only the hehavior the other play shoms spontaneously or is provoked into showing. Second, it must |xe alsk: to adjust its own behavior to exploit a represemative that identified as an exploitable player. Third. and perhaps mom difficult, it must be able to acheve this discrimination and exploitation without getting into too much trouble with the blleer representatives. This is something that mome of the rales arigmall submitred to the tourname'nt n'ere' able to do.

These very effective rules evolved by broking the num important device developed in the computer tournambnt, namely to be "nice," that is never 10 be the first to defiect. These highly effective rules always defect on the very first move, and sometimes on the second as well, and use line choices of the other player to discriminate what vould $\left.\right|^{x}$ done next. The highly effective rules then havt responses that allowed them to "apologize" and get to mulual conperation with most of the unexploitable representatives, and lhey had different responses which allowed them in exploit a representative that was exploitable."

Axelrod demonstrated, with his use of genetic algorithms, that intelligem hehaviur that was not part of the initial problem setup could emerge naturally thrumgh an evolutionany process.

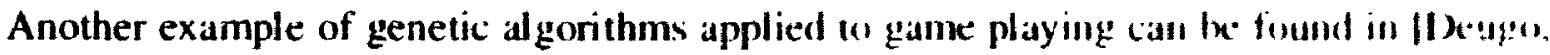
1989]. Deugo provides a framework for the integration of ('ase Based Keasulning wath Rule Based Reasoning and allows for the modification of cases using gentetic al gorithme. While the thesis provides a general framework for synthesis of ( axe Based Planning and genetic algorithms, its application to the game of checkess is of interest here lit Ixugos work the basic unit being manipulated is a case which represents is checkel stlategy. laach

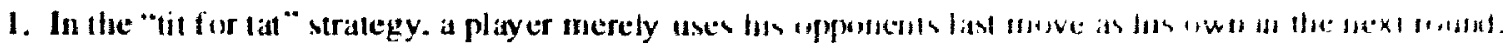


case has a collection of frames and a strength value. The checker case is just a sequence of moves experienced during the progress of a checker game.

(ienetic algorithms are used in the Simple Classifier System for Cases (SCSC) in order to improve the quality of strategies which are stored in the case library. The system works by playing a numbiar of simulated games which cause a library of cases to develop. At predefined points during the learning process cases which have been used successfully during the previous game playing cycle have had their value increased and so are preferentially reproduced in a modified case ljbrary to be used in the next game playing cycle.

The results of this thesis clearly show that integrating genetic algorithms with a Case Based Reasoning system can result in improved performance of the CBR system and that Ixthaviour not originally present in the case library, nor in any single acquired case. can imerge.

The Tower $O f$ Ilanoi is a well known problem. where rings of differing sizes are initially stacked in increasing dimension on one peg ard the goal is to move them to another peg where the rule that a larger dimension ring can never be placed on top of a smaller dimension ring. The lower Of Hanoi problem can be classed as either a game of strategy or a puzzle. As such it has frequently been of interest to the Artificial Intelligence community as a iest bed for problem solving algorithms. Cardoso |Cardoso. 1991| proposed solutions for a general number of rings using neural networks trained using the hackpropagation algorithm. More recently. Margarita [Margarita, 1993] has used genetic algorithms 10 solve the Tower of Ilanoi problem.

Margaritai approach to the Tower of Hanoi problem is interesting because it provides an exlended discussion on the importance of the fitness function and demonstuales hou genetic algorithm effectiveness is strongly influenced by function design!'. To quote Margarita.

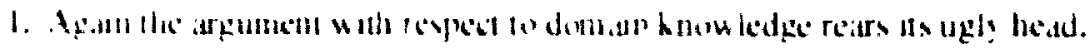


"The fitness landscape is the most important problem to consider when tuning a genetic algorithm ldeally. the fitmess function should have values monotonically increasing when going from one position to the next until reachung the final configuration. In other words. the tilness function applied to the optimal strategy should exhibit an increasing shape from the first move to the last so as to avoid the local maximal and surely converge to the final position during the evolutive process."

Margarita studied a relatively simple problem; that of 5 rings and a possible $6.18 \times 10^{1.4}$

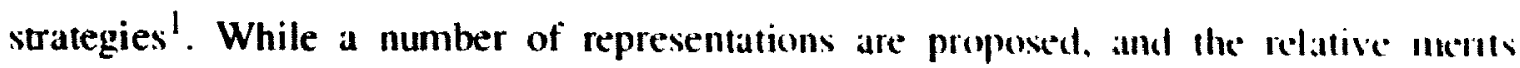
discussed, the representation used is one in which the string comprives the moves in the strategy. The coding of a single move is of the form $\left(D_{s}, D_{41}\right)$, where the sulscript s stanks for start and the subscript d stands for destination, and D the ring lo move. The corke usel for $D_{s}$ and $D_{d}$ is not based upon the absolute dimension but on their relative dimension. Considering just three rings - three possibilities exist - an alphabet represented by $(1,2,3)$ is therefore sufficient. The three rings present are of differing dinkensions, sis with no empty peg, it is always possible to label a smadlest. intermediate and largest ring. Similarly, if there is one empty peg (or two), the peg is defined as corresponding to the biggest ring (always bigger than the biggest ring). This encoding is shown in lable 4.

Table 4 Tower of Hanoi coding scheme

\begin{tabular}{|l|l|l|}
\hline Code & \multicolumn{1}{|c|}{ Move } & \multicolumn{1}{|c|}{ Meaning } \\
\hline 1 & (Min, Max) & Move the smallest ring on the top of biggest. \\
\hline 2 & (Min, Intermediate) & $\begin{array}{l}\text { Move the smallest ring on the top of the interme } \\
\text { diate. }\end{array}$ \\
\hline 3 & (Intermediate, Max) & $\begin{array}{l}\text { Move the intermedtate ing on the top of the big. } \\
\text { gest. }\end{array}$ \\
\hline
\end{tabular}

By u:: $g$ this encoding, a string can mever represent an infeasible strategy. The coding of a single action does not depend upon the previrus acturn. 'This is important because it ensures that no infeasible solutions are explored and that wo modifications are required to the crossover and mutation operators in onfer fo

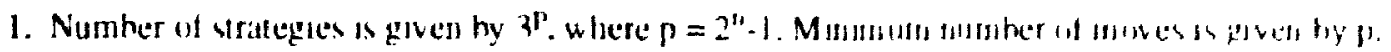


avoid the generation of illegal strategies. It can thus be seen that an effective representation, such as this one, can be used to significantly reduce the size of the search space which is explored. It can also be observed that this representation is only effective hecause the number of moves required to solve the Tower of Hanoi problem' is encoded in the problem itself and that the final solution represents the set of moves required to solve the problem.

It has been argued, with some vigour in [Rice, 1993] and by others, that strategies are not developed by this type of solution to the Tower of Hanoi problem in that generalization has not taken place. He argues that a higher-order representation, as embodied in Genetic Programming, is required in order to solve the n-ring Tower of Hanoi problem.

The investigation of fitness function choice provides the most interesting aspect if this paper. The three functional forms are shown below:

$$
\begin{gathered}
F=\frac{\beta\left(n_{2}^{2}+n_{3}^{2}\right)}{n_{1}+1}+1 \\
F=1+\beta n_{p} \\
F=\sum_{i=1} A(i . p) \times 10^{(n-1)}
\end{gathered}
$$

In the above equations, $n_{1}$ is the number of rings present on the pegs numbered 1 , 2 and 3: $\beta$ is an experimental parameter: $p$ is a peg number; $A(i, p)$ is a matrix with a number of rows equal tn the number of rings in the problem and with a numler of columns equal to the number of pegs that code the position of the

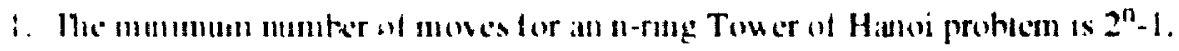


board. This last fitness function takes into acount not only the number of rings on the destination peg but also the correct position of the rings, assigning a weight proportional to the dimension of the rings.

It is interesting to note that Margarita criticizes the non-monotomic mature of his fitness functions and attributes the relative lack of success in finding an optimal strategy to their relative poverty in describing the actual fitmess landscapke. Actually, in most simulations, the genetic algorithm found a wimning stlatlegy in a number of steps far trom the optimal one but also far from the allowed onc - from one to a half to three times the optimal length although five timu's lac optimati length was allowed.

\subsubsection{Function Optimization}

Function optimization has most often been used as an arrea where modilicaltims in the basic genetic algorithm have been proven by evaluating potential changev against a kest bed of functions.

The earliest systematic work on function optimization and a use of fenctic algorithms is found in Hollstein |Hollstein. 1971/. Ifollsteins work correnued itself with optimizing functions of two variables using a varicty of luccoling schemes using animal husbandry and horticulture as their motaphon with lhe traditional operators of crossover, mutation from genetics.

The selection methods investigated by Hollstein are shown in Table 5 The mating preference methods can be seen in Table 6. In order to test the effects of combinanoms of selection scheme and mating method. Hollstein used a function test bed of 14 functions 
The computer runs used strings of 16 bits where two 8 bit strings were encoded as either a hinary unsigned integer or a Gray-coded integer.

Table 5 Selection schemes used in Hollstein's study

\begin{tabular}{|l|l|}
\hline \multicolumn{1}{|c|}{ Scheme } & \multicolumn{1}{|c|}{ Description } \\
\hline Proginny testing & $\begin{array}{l}\text { The fitness of offspring controls subsequent breedings } \\
\text { of the parents. }\end{array}$ \\
\hline Individual selection & $\begin{array}{l}\text { The fitness of an individual controls its future use as a } \\
\text { parent. }\end{array}$ \\
\hline Family selection & $\begin{array}{l}\text { The titness of the family controls the use of all family } \\
\text { members as parents. }\end{array}$ \\
\hline Within-family selection & $\begin{array}{l}\text { The fitness of individuals within a family is used in order } \\
\text { to control the selection of parents for breeding within a } \\
\text { tamily. }\end{array}$ \\
\hline Combined selection & $\begin{array}{l}\text { Here, two or more of the above selection methods is } \\
\text { combined to form a hybrid scheme. }\end{array}$ \\
\hline
\end{tabular}

As has been sten earlier, Gray coxtes provide the advantage that adjacent integers differ by a single bit. Hollstein also discussed the use of hash coding but did not present any results.

Table 6 Mating methods used in Hollstein's study

\begin{tabular}{|l|l|}
\hline \multicolumn{1}{|c|}{ Scheme } & \multicolumn{1}{|c|}{ Description } \\
\hline Random matırg & All mates are equally likely to mate with one another. \\
\hline Inbretiting & Related parents are intentionally mated. \\
\hline Line br zeding & $\begin{array}{l}\text { A uniquely valuable" individual is bred with a base pop- } \\
\text { ulation and their subsequent offspring selected as par- } \\
\text { ents. }\end{array}$ \\
\hline Outbreeding & $\begin{array}{l}\text { Individuals with markedly different phenotypic characteristics } \\
\text { are selected as parents }\end{array}$ \\
\hline Selt-tertilization & An individual breeds with itselt. \\
\hline Clonal propagation & An exact replica of an individual is formed. \\
\hline Positive assortive mating & Like individuals are bred with other like individuals ${ }^{\mathrm{b}}$. \\
\hline Negative assortive mating & Unlike individuals are bred. See footnote a. \\
\hline
\end{tabular}

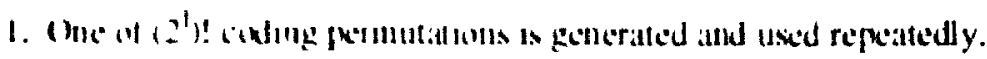


a. This is the anthesis of work done hy |Todh. Miller. |WoI|.

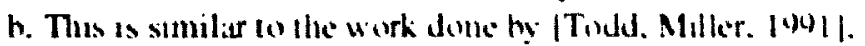

In all of Hollstein's work a population of 16 Mas used. He ohierved that this was too small a population and recommended that significantly larger populattions Ixused in future investigations of this kind. He noted that rccurrent inbrealing .llnd crossbreeding were the most robust of all bresding plans that he laned and that Gray codes provided some improvement over the unsigned integer reprexentaltuil lhus. he argued, was due to the natural way single bit mutations mapped to all intege' mencment or decrement.

DeJong's thesis |DeJong, 1975 | is considered pivotal in the history of gentetic al gorithmis. 'This thesis is considered important because it combines the whemata theorem with very carefully constructed computer experiments. DeJong construcked a test envirmmink al five problems in function minimization: taking care to include functions which had the following six characteristics:

1. continuous/discontinuous

2. convex/nonconvex

3. unimodal/multimodal

4. quadratic/nonquadratic

5. low-dimensional ity/high dimensionality

6. deterministic/stochastic (or noisy)

Actually, the five functions used in his study form the first live funclions encorled itl the GENEsYs' system and can be found in Appendix A. Function optimization was cloosen for his study of genetic algorithm performance in order in (a) remove the prohleoll of choosing a problem representation; thereby avoiding the problem of introlucing: representational bias into the study and (b) the fitness function is the func(usu itx.ll (possibly scaled). DeJong was interested in characterizing the effectiventess of vallous genetic algorithms and introduced the concepts of on and off-lime perfomance. ( $n$ lane performance represents the ongoing performance of the genetic algorithm whereas offliur. performance represents the convergence level for the al gorithm.

1. GENESY s due to Back) is a derivative of the GENESIS (due fo (iretentelte) 
Ix:Jong's studies investigated six reproductive plans, found in Table 7.

Tahle 7 DeJong's Reproductive plans

\begin{tabular}{|c|c|}
\hline Man Name & Pan Description \\
\hline R1 & $\begin{array}{l}\text { Roulette wheel reproduction. Individuals reproduce in } \\
\text { proportion to their fitness relative to the average fitness of the } \\
\text { population. }\end{array}$ \\
\hline $\mathbf{R 2}$ & Elitist model. Best structures are preserved. \\
\hline R3 & $\begin{array}{l}\text { Expected value model. The expected number of off- } \\
\text { spring are created in the next generation. }\end{array}$ \\
\hline R4 & Elitist expected value model. A combination of $R 2$ and $R 3$. \\
\hline H5 & $\begin{array}{l}\text { Crowding factor model. When an individual reproduces, } \\
\text { a similar (as measured by the number of bits the same) } \\
\text { individual is randomly selected to die. }\end{array}$ \\
\hline R6 & $\begin{array}{l}\text { Generalized crossover model. This is equivalent to a } \\
\text { multt-point crossover operator. }\end{array}$ \\
\hline
\end{tabular}

The majorily of investigations occurred using the $R$ I reproductive scheme with conventional single point crossover and mutation operators.

\subsubsection{Neural Network Optimization}

The Iraining of neural networks has been shown to be a hard problem, and the selection of an appropriate architecture for the task domain to be a black art. Even small networks can behave in ways that defy comprehension; multi-layer, non-linear networks have no intuitive interpretation. No comprehensive analytical or algorithmic solutions are in sight and empirical results have shown Io te limited in terms of their generalizability. Many different classes of architecture for ileural networks have been documented and for a good overview the reader should consult Simpson [Simpson. 199(1).

(ienetic algorithms have been used to design neural network architectures and determine the weights associated with the links between neurons. The application of genctic algorithms to the problem of neural network design is appropriate for a number of reasons. Firstly, the space of possible architectures is very large and analytical results do not exist for the selection of an architecture appropriate to 
the task. As a result of the size of the space of possible architectures. mublished accounts of architecture investigation have tended to dkal with simple

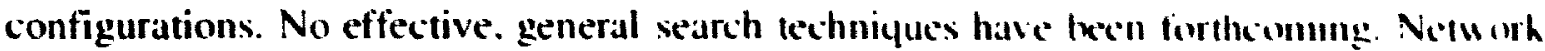
parameters also show strong interaction - implying highly nonlintalt Ix-hat intul - givmg rise to local minima which tend to deceive heuristic search and elementary oplimusation techniques. Equally importantly. most applications have to deal with noisy data which wan cause the failure of even the highly robust backpropagation algorithm!

A number of issues present themselves when using genetic algorithms for mounal network design. Firstly, the problem of representation needs 10 ix addressed. II the case of fixed architecture. how many bits are required in ordet II reples.llt the weights in the network effectively" In the case of a dynamic anchitcoluse, what data structures can be manipulated genetically in orcler lo desgul the most effective neural network for the task domain? Secontly, what adaptations of the genetic operators are necessary for the genetic mantpulation of neural networks. These questions, and steps towards answering them, can in foumd in |llarl., Sumad and Guha, 1989].

The genetic neural network design problem can be broken down into two callegoric's. These are genetic algorithms that attempt to generatc the weights for a given althitectuse (the static problem) and genetic algorithms which induce the architecture as well (1// dymamic problem). The second class of problem is naturally considerably harder. 'Ihe t irst problem has received considerably more attention than the second for that reasm. So far.

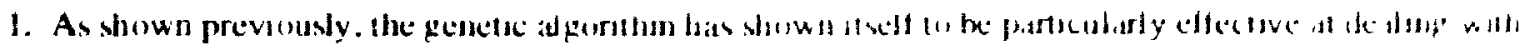

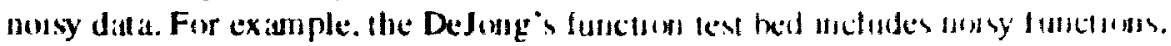


c,nly feed forward network design has been attempted. The basic architecture for genetic algorithm design of neural networks is shown in Figure !?.

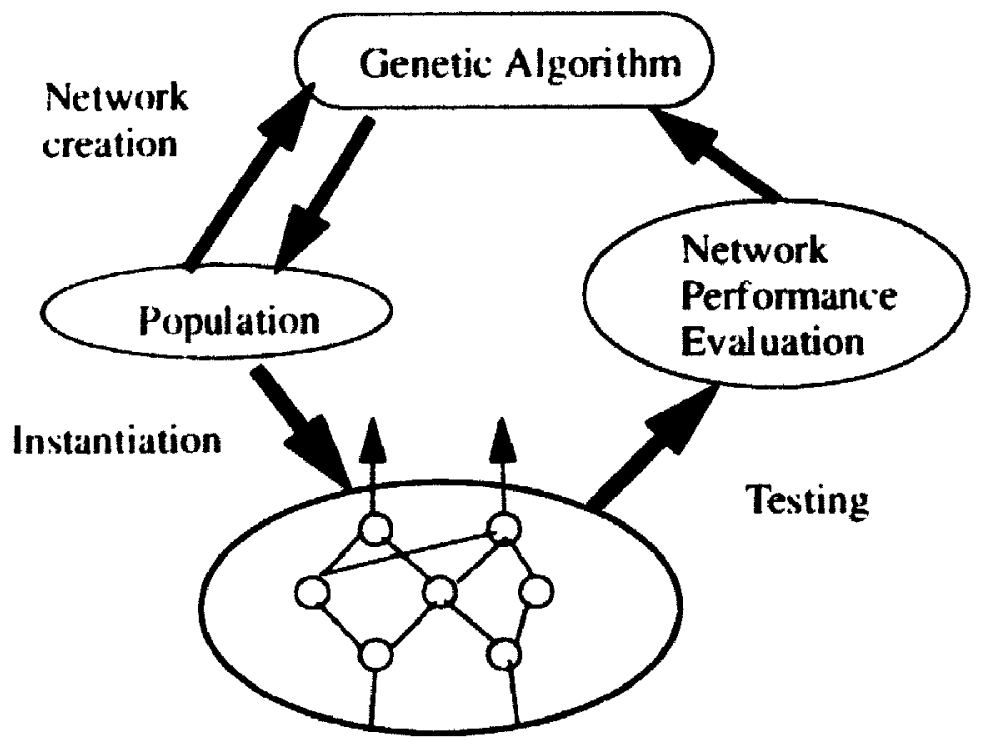

Ac:ual Neural Network

\section{Figure 10 (ienetic Algorithm Design of Neural Networks}

Both categories of design problem use the above methodology.

\subsubsection{The Static Problem}

In the static problem. genetic search attempts to replace learning (such as the Backpropagation algorithm) as the mechanism for finding weights in fixed architecture neural networks. Here, the bit string representation for the neural network is straightforward and easy to implement. For example, IMiller and Toxd. |989|. |Whitley and Manson. 1989| and |Montana and Davis, 1989] have compared genetic search to gradient descent learning for particular network designs and problem domains.

Early work in this area concentrated on the recombination of binary encoded strings to predict weights in snall neural networks. However, results were mixed and found difficult to extrapolate to pioblems requiring more than $3(x)$ bits. It is 
believed that the reason for limited success was duc, in part. to the cevistence of multiple symmetric weight-set solutions. Restated. this says that the solution space contains multiple maxima and the crossover operator is constantly lorcing: us to move towards different peaks in the solution space - oxillation then incurs.

However. work by Whitley, et al |Whitley et al. |(4)|| appears to have ancicome this problem. Firstly. in their implementation of the genetic algorithm for ncural me-1worh prediction they use real-valued weights instead of binary encoding and thit the crossover operator only works on weight boundaries. In a sense, they are no longer working with a binary alphabet but rather the alphabet of real numbers. Secondly, a much higher mutatum rate is used, this being necessary owing to the significantly reduced exchange of gerenctic information between population members during crossover. An example of crossover an real-valued boundaries is shown ia: Figure 11.

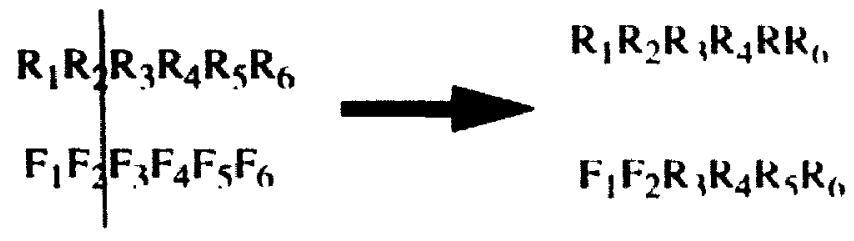

\section{Figure 11 Crussover on real-valued boundaries}

This is in direct contrast to a more traditional genetic algorithm where the mutation operator is considered a "secondary" operator and in some work, |Ko/a, | al together.

Finally, a small population (typically 50) is used in the genetic search. This, the autlors state, has been found to reduce the exploration of multiple solutions which atte representationally dissimilar. By relying more on mutation than on the more perwerful crossover operator, the genetic algorithm becomes more of a genetic hill climile. During the genetic search process, the size of the population is gradually reduced until a single population member remains. This is in stark contrast to a conventional genctic alyenritum in which the population size remains constant ${ }^{1}$.

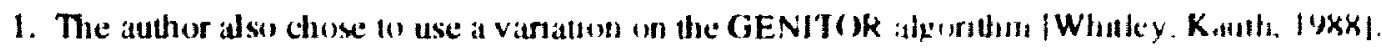


In |Whitley et al, 1991| a genetic algorithm is used to generate a network which controls an inverted pendulum, or broom-on-a-cart and the results contrasted with those generatel using an Adaptive Heuristic Critic (AHC) network'. The genetically engineered networks were :hown to have better average worst and best performance when compared to the traditionally-trained AHC networks. Although search time was considerably reduced hy decreasing the populations size during the course of genetic search. it had the unfortunate side effect of increasing the probability of failing to find a solution.

\subsubsection{The Dynamic Problem}

Automatic mechanisms for node creation and/or destruction in neural networks, ixcurring maturally during the learning phase, are a relatively new phenomenon |Ash, |988|. This is because the problem of generating both weights and architeclure of a neural network is considerably harder than the static problem. This is so because of the difficult representation issues involved. At least three distincl approaches have been documented [Harp, Sumad and Guha, 1989]. |Kitano, |qx|| and |Koza, 1941, pgs 81-88|.The focus of the work of Harp et al is the gentration of the architecture of the neural network, the weights being computed using the Backpropagation algorithm. Between each generation of the genetic algorithm, a neural network builder takes the candidate strings and builds a neural network which is subsequently trained using the Backpropagation algorithm. As the authors noted, automatic weight generation could be included in their representation by encoding the weights directly in the bit string. In /Harp. Sumad and fiuha. 19891 a bit string representation as shown in Figure 12 is proposed.

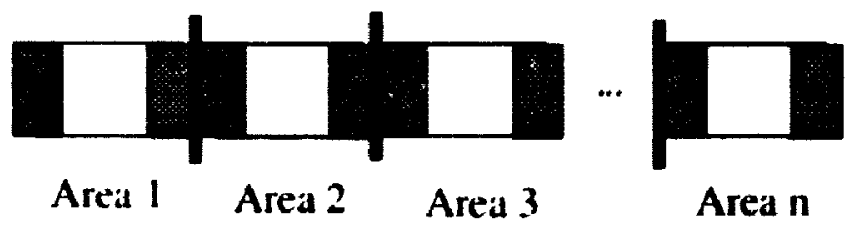

Figure 12 (Werview of neural network bit string representation

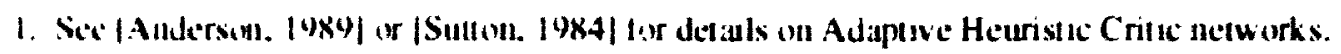


The bit string is composed of several segments called areas. Fath area contains Iwo structures, an Area Parameter Specification (APS) and a Propection Specification Ficlil (PSF). These areas contain information on its connectivity to other areas and informaltion on the area itself. A more detailed view of the suructure of an area is vomn in figure 13 below.

Area

Paranineter

Specification

Projection

Specification

Field ( $(0$ or more)

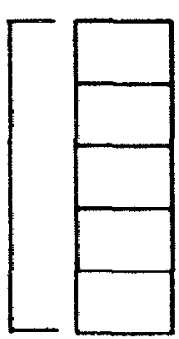

The area ID, an address:

Total Size

Dimension I share

Dimension 2 share

Dimension 3 share

Stan of Projection marker

Target address

Mode of address (absolute or relative)

Radius, Dimension 1

Radius, Dimension 2

Radius, Dimension 3

Connection Densiry

Initial Eta

Eta slope

End of Projection marker

\section{Figure 13 Area and Projection Specification freld detail}

The markers in this representation enable (a) a modified crossover operatol (1) fellilate new networks that are structurally viable and (b) there is a well-kefined mapplag (16 a neural network. Harp et al limited the crossover operator to genetic exchanger al arrat borders only. 
Fach substring within a given area consists of a number of bits between 0 and 4 - in their experiments 3 was always chosen. Parameters are Gray scale encoded in order that values close in the chosen domain are close in bit string representation. An area has a total size which determines the number of units an area will have. This ranged from 0 to 7 . Eta and Eta slope are used to govem the initial learning rate used by the backpropagation al gorithm and are designed to represent 8 val ues in the range 0.1 to 12.8 .

The APS total size parameter represents the number of neurons in the area of the neural network; the value being interpreted as log 2 .

The APS is used to define the sizes of the various areas in the associated projection specification field. Being a three dimensional neural network representation, the three "Dimensional share" values are required. Dimensionality was included in this "general" representation in order to support the discovery of neural network architectures usable in vision pattern recognition problems.

Each PSF indicates the target area - two modes of addressing being supported. Firstly, alswhtute addressing can be specified: in which case the linkage is to the area indicated by the target address value. Secondly, relative addressing can be specified in which case the linkage is to the area specification address plus the value indicated in the target address structure of the PSF. Relative addressing is considered necessary in order to limit the destrucliveness of the crossover operator.

The radii structures in the PSF allow units in an area to project only to a localized group of units in the target area. This feature, the authors believe, allows the target units to have lowalized receptor fields, which is common in biological neural networks and extrencly important from the viewpoint of hardware implementation.

The connection density structure provides a measure of the connectivity of meuron between areas. Eight values are provided, ranging from complete comectivity to 30$) \%$ connectivity.

1. Dic semantics of aero are the next area. 
Harp et al, do not consider this representation ideal. In |Harp a al. |Wist) they state:

"The genetic operators tend to rip apart distant comncctions an strings: it is useful to cluster interacting genes to increase the likelihood that successful combinations will tavel logether."

Although this thesis does not consider the neural network design publem III detail. the above quote almost hegs of have the adaptive comsomer "peratlu applied to the above problem in order to have the adaptation identity which gentes are interacting strongly and ensure crossover of these fightly-coupled genses.

Harp et al describes the representation applied to the fis digut rovenutun problem and shows that the genetically enginecred networks are comvideially better than generating networks randomly.

Another approach to the neural network design problem has alwo concentialled upon architecture determination. This approach, due to Kitamo |Kitano, |以M|, owes its inspiration to Lindenmayer systems, or L-systems, Himkenmayer. IW(ix. 19711. Kitano's approach differs considerably from Harp al al, ill thatl llec architecture of the neural network is not dircefly encoled in the bit string but rallee in a graph generation graınmar which is used to generate the neural network anchitecture.

Kitano criticizes the previous genetic algorithm-based approaches we newal netwonk design for two reasons. Firstly, results on the scalability of their approaches have now bern forthcoming. In fact. Kitano demonstrates the scalability problems for is simple e.ticunleo/ decoder problem using the approach documented in |Miller and Finkl. |Mx)|. Accuracy (i) the neural network designs is shown clearly to degrade vigniticantly with increaving: network size. Secondly, that the implementations are biologically infeasible as llicy inply that connectivity information is encoded in DN $\Lambda^{!}$.

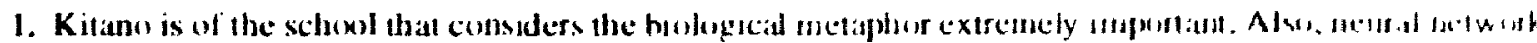

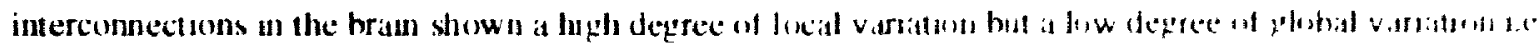

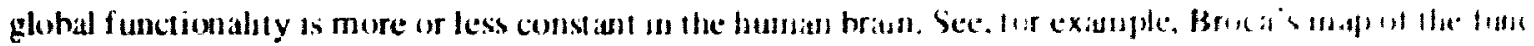
inolity of the human bran. 
Kuano $>$ graph generation scheme is defined as $L=(S N, S E, M N, M E, R N$. RE, (1)). The maning of the various symbols is show in Table $x$.

Tahle X (iraph Generation Scheme Symbols

\begin{tabular}{|l|l|}
\hline \multicolumn{1}{|c|}{ Symbol } & \multicolumn{1}{|c|}{ Meaning } \\
\hline SN & A set of symbols representing the state of a node. \\
\hline $\mathrm{SE}$ & A set of symbois representing the state of an edge. \\
\hline $\mathrm{MN}$ & $\begin{array}{l}\text { A set of } 2 \times 2 \text { matrices with symbcls for nodes assigned at diagonai ele } \\
\text { ments and a symbol for an edge assigned at non-diagonal element }\end{array}$ \\
\hline $\mathrm{MF}$ & A set of $2 \times 2$ matrices with 0 and symbols for edges \\
\hline $\mathrm{RN}$ & Rewriting rules for nndes. \\
\hline $\mathrm{RE}$ & Rewriting rules for edges. \\
\hline (1) & Initial graph. \\
\hline
\end{tabular}

By application of the graph generation system, a strong' representation of the neural network |Miller and Todd. 1989) is generated. This is demonstrated in Figure 14 and Higt:re 15.
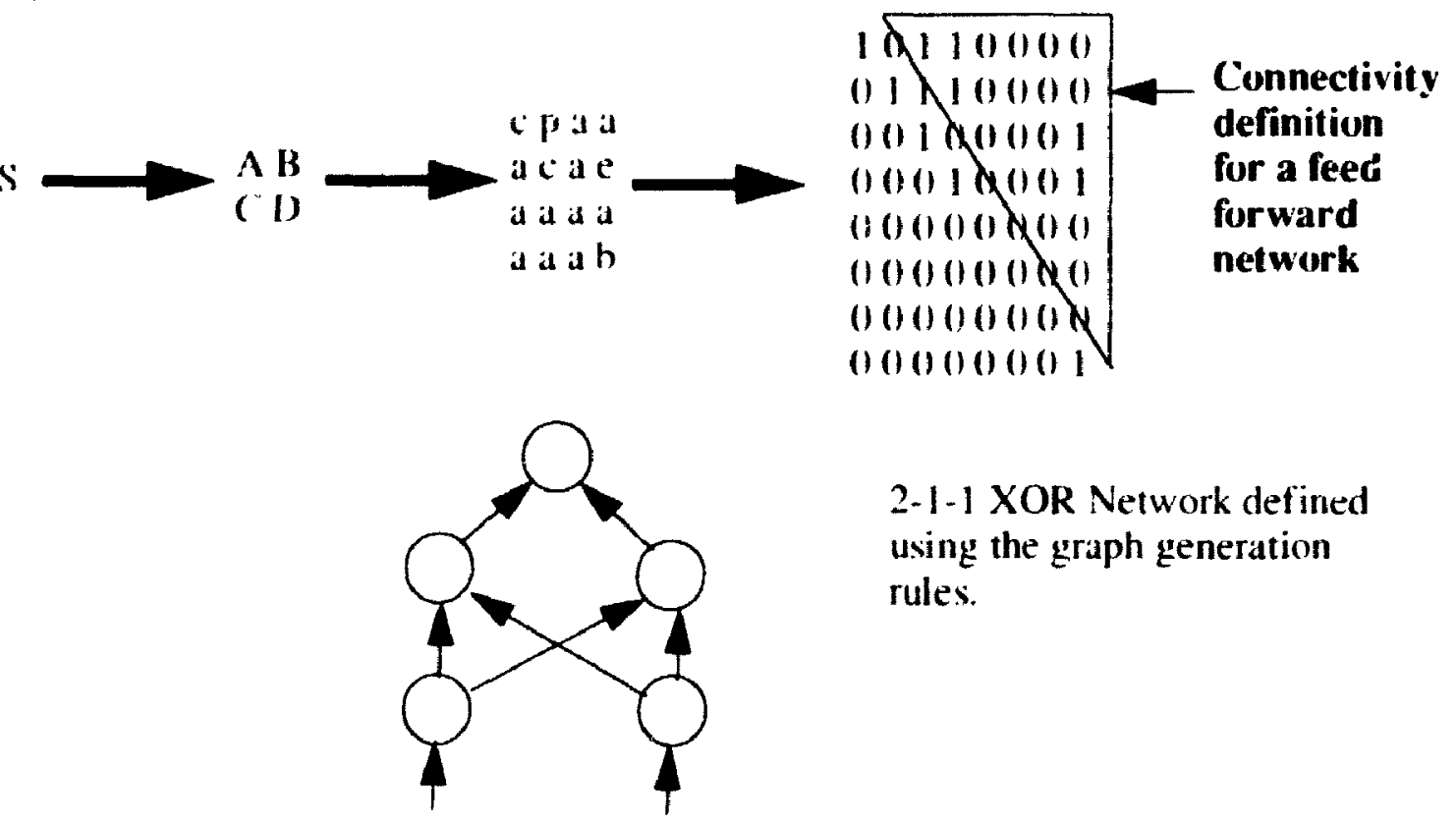

2-1-1 XOR Network defined using the graph generation rules.

Figure 14 Demonstration of (iraph (ieneration Grammar for XOR

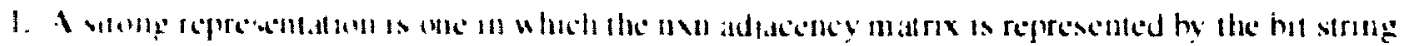



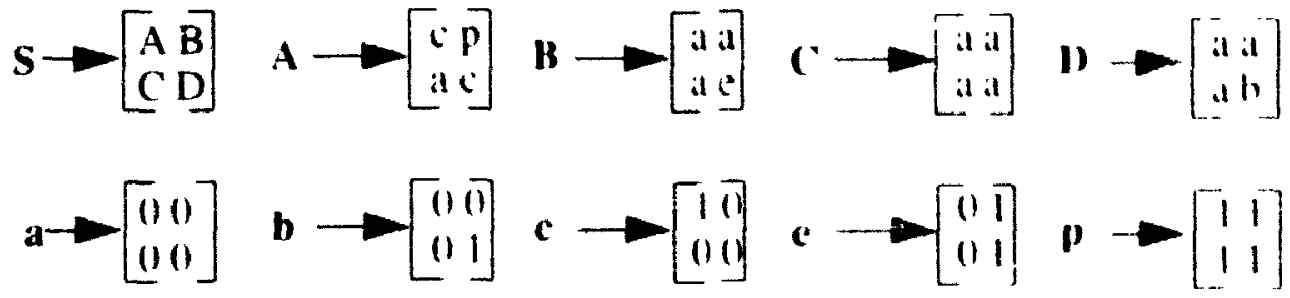

\section{Figure 15 (iraph generation rules for 2-2.1 X()R network}

The encoding of graph generation rules in a chromosome comsists of a tarialle. portion and a constant portion with crossover and mutation eprealtiuss leving limited to the variable region of the string. The constant portion of the stim! represents the left hand side of a graph generation rule, the variable part the ferellealled production. An example is shown in Figure 16.
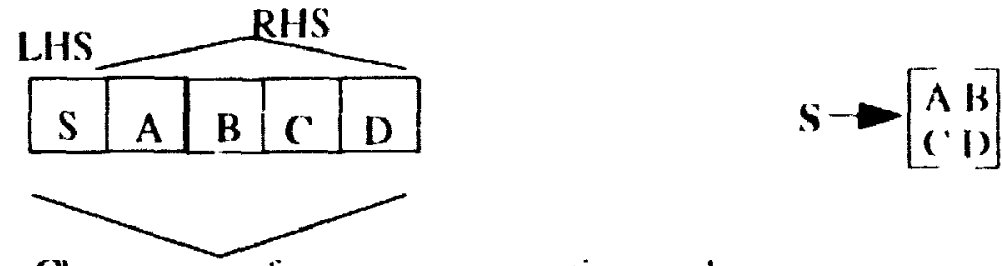

Chromosome fragment representing a rule

\section{Figure 16 Chromosome encoding grammar}

The constant part does not change, and the 16 rules that rewrile symbols foll 1 moll differentiated cells into symbols for differentiated cells are comerked hert. Hals rule consists of five alleles (as shown in the figure above). Ion the valtable region, symbols are generated for each position in the chromosome th the tallge $A$ of $p$. The initial chromosome fragment is fixed to be $S$, the initial graph, in orden that the imtial graph can always be evolved (i.e. a rule exists which transfomms the intial gtaph).

The chromosome rules are used to generate neural network by the process of sewillm! symbols until no further symbols can be rewritten i.e. the adjacency matrox a compunted. 
The results of using this representation on $4-x-4$ and $x-x-x$ encoder/decoder problems indicated superior accuracy and scalability when compared to /Miller and Fodd, |989|. Kitano attributes this to three things. Firstly, the grammar cncoding method generates more regular patterns than the direct encoding method. This is extremely attractive from a biological perspective as comsiderable regularity is observed throughout the brain. Secondly, the grammar encosling method is better able to preserve the meaningf sl subcircuits which are discovered during the genetic algorithm. This latter point is in direct contrast to the direct encoding method where connectivities are distributed throughout the chromosome. Reuse of a useful neural network fragment is not possible. Finally, the grammar encoding method requires considerably shorter chromosomes when compared to the direct encoding method - where the adjacency matrix is directly manipulated.

Finally, in |Koza, 199()|, the problem of neural network architecture and weight determination has been approached. In all of Koza's work, LISP S-expressions are manipulated using a genetic algorithm with special crossover and mutation operators. In the neural network design domain, the function set that Koza uses consists of $F=$ IP,W,t,* / . The function $P$ is the linear threshold processing function. The function $W$ is the weighting function used to give a weight to a signal going into a P-type node. It is, in fact, multiplication. The set of terminals that is used consists of $S=\left\{D_{1}, D_{2}, \ldots, D_{n}, R\right\}$. The $D_{1}$ represent the input signals and $R$ is a random number. An example of Koza's reprexentation for a neural network and its conventional representation is shown in Figure 17.

While the genctic algorithm is applied in a conventional way, the manipulation of the IISP S expressions that represent neural networks require special rules of construction. The loct of the tree has to be an L-node, or list node. Immediately below this node, one or more ' l nodes must be present. At a level below a P node. W nodes must be present. At the level belou a weighting node, it is possible to have an arithmetic operator, an input signal. a randon number or another $P$ node. The neural tree is generated by applying these rules 
recursively until a depth limit is reached, or a full tree is generated. The leall es of the tree are always floating point values or input signals.
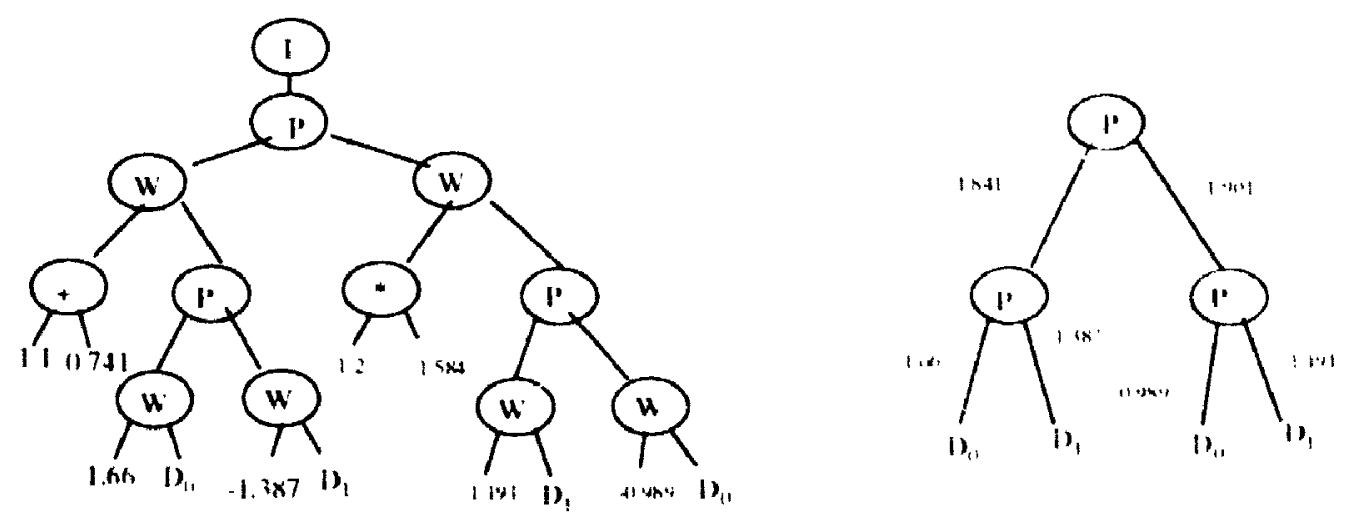

\section{Figure 17 (ienetic Programming and Neural Networks}

As can be seen, this representation does not place any limits on either the archilecture or weights of neural network connections. This representation and use of gentetic algorithms provides the most general mechanism for neural network induction dixumented to datte. The learning associated with the neural network takes place exclusively by genelic search from one generation to another.

The crossover operator takes two members of the population and, selecting modes of the same type, exchanges subtrees. This is actually unnecessarily restrictive but did not appeal to degrade ${ }^{1}$ the results of the genetic search. By selecting nodes of the same type tor crossover, we guarantee to generate a structurally correct neural network. Simmlally, the mutation operator selects a node within the tree at random and replaces the nomke with a randomly generated subtree subject to the previously described rules.

Koza has used the above representation and modified genetic operaturs morden w ile vell neural networks that solve the XOR problem ${ }^{2}$ and the two bit adkler problem. His results, using no mutation, and an initial population of $5(x)$, indicate that there is a $40 \%$ chathe of

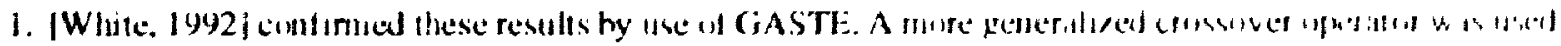
in GASTE.

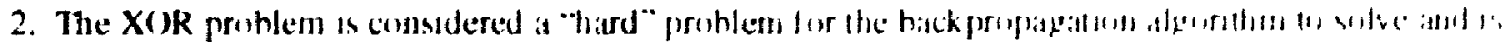

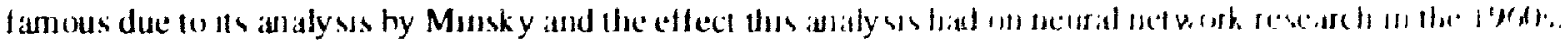


generating a $1011 \% \%$ correct individual within 3 independent runs of 50 generations - a very impressive result!

\subsubsection{Design}

A wide range of design problems have been solved using genetic algorithms. In this rection, design in the area of telecommunications is considered.

Communication network design is a difficult process. A wealth of literature has bxen devoted to the subject, with the development of a large number of heuristic algorithms being proposed. Books by Kleinrock |Kleinrock, 1975], Tannenbaum |Tamnebaum, 1981| and Bertsekas and Gallager |Bertsekas and Gallager. 1987| provide excellent sources for such material.

A related problem class is that of optimization of network operational parameters in order 11) minimize average network delay. In a DPN ${ }^{2}$ network, traffic is routed deterministicall; from a particular source to any given destination. However, many equivalent paths may exist from which to choose. Without explicit human intervention the network will make less than optimal routing choices as a result of having an incomplete view of the network. The manual specification of preferred path service data for a DPN 100 network presents such an optimization problem and traditionally has been seen as a time consuming process. Preferred path service data is used in a DPN $1(1)$ network in order to balance the utilization of available trunk capacity and to route traffic in such a way as to reduce network delay. In a large network, with many equivalent paths from which to choose, this optimization problem becomes infeasible for manual solution. The probability of being able to balance the utilization of the backbone network by making primary path selections manually is low and the time taken to do it prohibitively high.

An algorithm based upon hill-climbing techniques has been implemented in crice to solve this problem. The algorithm used to compute primary neighbor sclections is based upon first generating a feasible (unbalanced) solution - the

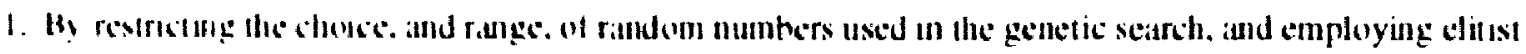

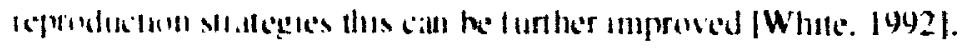

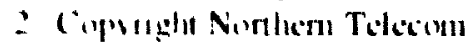


Mininum Link algorithm |Chou and Frank, 1972] - and then perturbing this solution in order to provide a more halanced network - the How 1)(iattun algorithm [Fratta et al. 19631. This hill climbing algorithm continues until ather no more improvement can be seen or a given number of iterations arce completed.

Unfortunately, the hill climbing approach does not guarantere the genteratlun af a globally optimal solution. Being a hill climbing algorithm. if the space of fousible solutions contains many local maxima, the algorithm will most likely comverge lo the top of a local peak rather than find the global maximum. This is a highly undesirable characteristic of the algorithm.

The purpose of primary neighbor selection is to Iry and balance the utilization of trunk capacity within the networh by making choices for preferred pall illmulys equivalent paths.

The primary neighbor problem (PNP) cannot feasibly the solved manually for large. networks witt, a reasonable number of paths from which to choose. For example, in a large european network, approximately $2^{64}$ networks can be defined by choice of primary neighbor. This european network is relatively small; consisting of 34 modules and 62 trunks. Even evaluating one network per second, a total of $5.8 \times 10^{11}$ ycars would $\mathrm{k}$. required to test all possible combinations for primary neighbor welection. (Ahvunsly. a solution strategy is required in order to search the solution space more efficienty. The process of specifying routing parameters using a genetic algorilln! is slown diagrammatically in Figure 18 below.

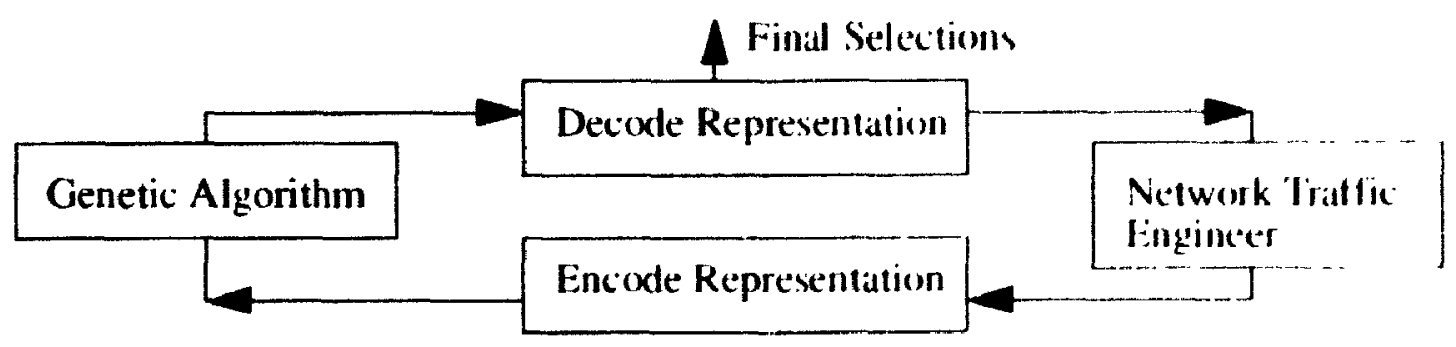

Figure 18 The (ienetic Algorithm Primary Neighbor selectiun process 
()re of the overriding concerns in choosing a representation for this problem was that the representation not allow the expression of infeasible solutions. For example, if only two possible choices exist for primary neighbor selection and the representation allows for four this would be an error. In other words, the representation only defines the space of feasible solutions.

By refuiring the above, it became necessary to choose a non-binary alphabet for chromosome expression. Consider two nodes, PM DKI and PM CH2. PM DKI has two primary neighbor selections, each with two choices. PM $\mathrm{CH} 2$ also has Iwo primary neighbor selections, one with two choices, the other with three choices. Thus if we were to use a binary alphabet for expression of the (partial) solution, PM DKI would require $1+1$ bits while $\mathrm{PM}$ CH2 would require $1+2$ bits. Ilowever, in the latter case, while only three possibilities exist, the representation allows for four. An inforathe solution is allon'ed by the representation.

A natural representation is to use an alphabet which depends on the number of choices to the made for a particular PM and then modify the mutation operator appropriately. So, using the previous example. PM DKI uses a $\{1,1\}$ alphabet for the choices available to it while PM CH2 uses a $\{0,1\}$ alphabet for the two choice primary neighbor node and a $\{(1,1,2\}$ alphabet for the three choice primary neightor node.

It is also important to realize that the representation can only be computed once The routing lables have been created for the network as it is only at this point that the number of possible primary neighbors is known. The overall representation for the chromosome to be used $i_{12}$ the genetic algorithm is then derived as follows:

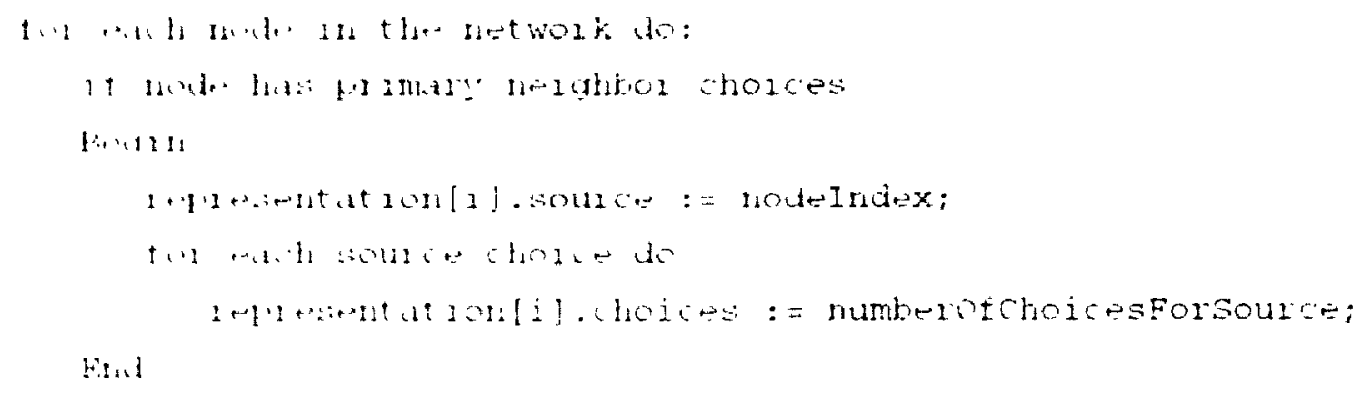


The representation/il.choices information is used in order 10 (a) gerlleralle in initial population and (b) used during mutation in order to ensure that the valte for a specific gene is changed to a legal valus. The maximum number of chomes at any point is maxTrunkGroups (31), which can be encoded in 5 his. Ilenter. Ihe physical size of the chromosome in bits is just the number of moks line which primary neighbors can be defined (source to destination) multiplied by fivc.

Note: The physical implementation of the represintation is vill a bit string. It is only the logical implementation of the chromosimic which uses variable, possibly non-binary alphabets.

The crossover operator needs in modified such that crossover anly incius an live bit boundaries.

The modification to the mutation operator is a little more complex date lit Ile variable alphabet used throughout the chromosome. The cokle fragment ix.lun applies to the ith gene in the chromosome.

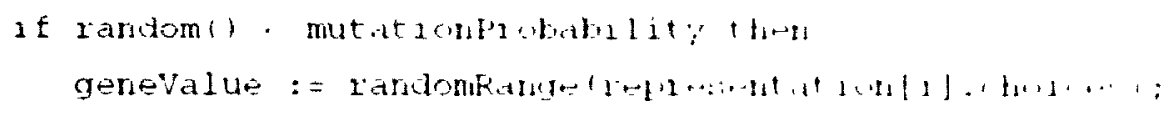

The above code fragment modifies the geneValue 10 lo in the lange of cro to representation|il.choices - 1. Note that the value may for unchanged as llue function randomRange() returns an integer chosen using a unitorm disuibutioul. This is a little different to the previous definition in which a different vallue was guaranteed. Actually, the difference in practical terms is resolved by rickeasul? the mutationProbability.

The fitness function for the primary neighbor problem need 10 be able to lahe into account constraints specified by the user. Certann trunks might med 10 Ixutilization limited and solutions generating values in excess of these threstmols 
be considered highly unfit. For this reason a penalty must be added to those chromosomes which exceed user specified thresholds. In the equations below, this is represented by the penaltyfitness, and the penalty value in the same equation is an algorithm or program parameter.

It might be useful for networks with primary neighbor selections which violate user defined constraints to have a non-zero fitness i.e. they can appear fitter than some networks which have highly unbalanced trunk utilization values. This is designed into the fimcss function included here and can be controlled through the setting of the ponalty parameter. The Comstraintliil, values are also program inputs. For trunk groups that are not utilization limited, the (omstruint tril $_{j}$ value would be set to $10(0)$ in order to ensure a zero penalty term. The Util $_{\|}>$ConstraintUtil ${ }_{j}$ term returns 1 if true, $(1$ if false. so that the term (l/til $\left.\right|_{1}>$ Constraint Util, ) penalty returns either 0 or penalty.

The index $\mathrm{j}$ in the above equations refers to the $\mathrm{jth}$ chromosome in the population. The $0 .(11$ figure in the final equation is a rounding term only and consures that the fitness of any chromosome lies in the range $(0,1(1))$. In the equations below, all utilization values are measured in the range (0,1).

The results ohtained by implementing this search strategy compared to the hill climbing mothod proved that the genetic algorithm did not tend to be trapped into

$$
\begin{aligned}
& \text { (1)iall rurdo- }
\end{aligned}
$$

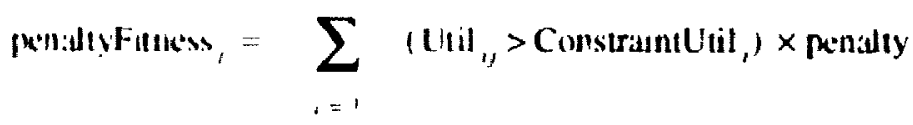

$$
\begin{aligned}
& \text { marl rum, } \\
& \text { liil }_{\text {an! }}=\sum_{i=1} \text { Ulil }_{t /} / \text { totalTrunks } \\
& \text { whali runks }
\end{aligned}
$$

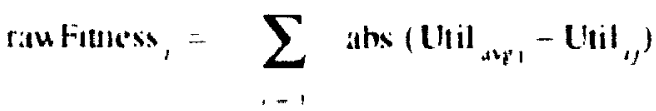

$$
\begin{aligned}
& \text { lotalkawFiness, = rawFiness, + penallyFiness, }
\end{aligned}
$$

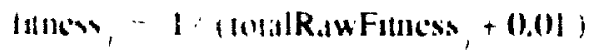


producing a local minimum. Other use of the genetic algorithm in the allea it Telecommunications can be found in |Commbs. Davis. |OS7|. |Davis. Commbs. $|487|$ and [Pan. Wang. 1991|.

\subsubsection{Automatic Programming}

It has long been a dream that one day computers will gemerate pungams automatically. As such, a number of attempts to generatc programs atulnmallacally using genetic algorithms have been made. Not all of these efforts have

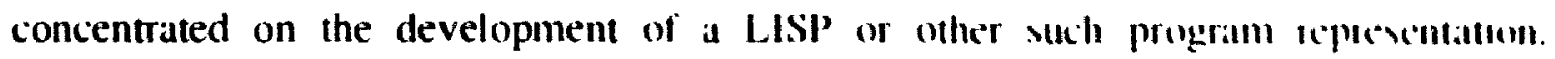
Finite state machines have also been chosen as the following paraglaphs shum.

An early evolutionary programming attempt used small finite statte machimes flengel el al. 1966). In this work, sequential symbol prediction tasks were perfomed by siaching: through a space of small finite state machines. The purpose of the makinkes was la identify patterns in symbol sequences which were prexented to theol. Foggel at al. IIscal only selection and mutation operators in their work and pertommed only single mutations in moving from parent to offspring. This, essentially random, search of the spakce al tinite state machines proved to be inadequate for all but the smallest problems. No viluclumed recombination operators were present in their evolutionary programming effurts.

The single-step mutation operator had an interestumg dexription floget il al. 1966, pg. 14-15!:

"An offspring of this machine is then proslucied throught mutation; that is, through a single modification of the parent machine in accordance with some mutation noise distribution. The mode of mutation is determmed by the interval within which number selected from a random number table lies. The intervals are chosen in accordance with a probability distribution over the permitted meskes of mutation. Additional numbers are then xelected in ordet in determine the specific details of the mutation. Thas the offspring is made to differ from its parent either by an cutput 
symbol. a state transition, the number of states, or the initial state."

The lack of structured recombination and an acknowledgment that the work applied to vmall finite state machine problems tended to see this work Ignored by the A.I. community. Further evidence for the limitation of a "structured hill-climbing" approach to the induction of finite automata can be found in [Tomita, 1982].

However, a related piece of work due to Zhou and Grefenstette [Zhou. Grefenstette. 1986] uses the full power of a genetic algorithm in order to induce descriptions of finite autumata. These automata learn to parse a regular expression which is presented to them via positive and negative examples - an NP complete problem /Gold. 19781. The automata thus learn on the hasis of incomplete information. The automata are assumed to have a maximum of $X$ states and are capable of parsing strings composed of a binary alphabet. The search space considered was large $\left(10^{22}\right)$ thus answering one of the limitations iimplicit in fongel et al's work.

The representation used was of the following form:

$\left(\left(X_{1}, Y_{1}, F_{1}\right),\left(X_{2}, Y_{2}, F_{2}\right) \ldots\left(X_{X}, Y_{X}, F_{X}\right)\right)$

where each $\left(X_{1}, Y_{1}, F_{1}\right)$ represents the ith state of the automaton. $X_{i}$ and $Y_{i}$ correspond to the destination states of the $\|$ and 1 transition respectively. $F_{i}$ is represented by three bits: the first two of which indicating whether or not there exists an arrow coming from the ith statc, and the final bit determines whether the state $i$ is a final state. Hence, the string reprexentation for this problem is $(3+3+3) * 8=72$ bits. The search space is thus $2^{72}$ in size. Small finite automata were preferred over larger solutions as suggested in /Michalski. I1) 8.31 in order to induce parsimonious solutions.

The results of this work are quite convincing, even given the fact that the size of the automaton which can be generated is small (being only eight stutes). Correct finitc state automata were developed after a small number of generations. The paper contrasts the genetic approach with random search and clearly demonstrate that random search fails to identify the finite state automata. The scalability of 
this work is uncertain. This author has worked directly whth a musc natural representation - that of graphs |White. [1642]. A moditied cromover operator. exchanging strongly connected componems in parent graphs, and a mutation operator which either added or deleted a nokle. allowed cyuisalcur finite statto machines to be generated. However. the approach did not walle well.

Other work in the area of induction of finite statc antomata $(I S A)$ cian Ix found in |Jetferson et al., 1991]. |Collins and Jefferson, |991|. In this work, finite state atumitta are again encoded in bit string format; in this calse several hundred bits long. The pupuse of the Jefferson work was to develop an FSA capable of following a trail a findting: problem. The trail has a series of turns, gaps and jumps that get more and more ditlic ult as the trail progresses. The experiment was an allempt to so if 11 is possible to prokluce

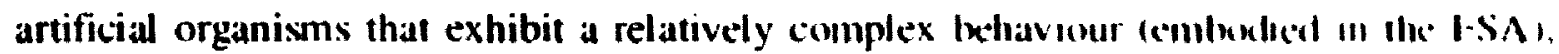
and to do so by evolutionary methods. with no built in design blas and wilh mo leaning:.

Table 9 Binary-coded State Transition Table

\begin{tabular}{|l|l|l|l|}
\hline Old State & Input & New State & Action \\
\hline 00 & 0 & 00 & 01 \\
\hline 00 & 1 & 01 & 11 \\
\hline 01 & 0 & 01 & 01 \\
\hline 01 & 1 & 11 & 11 \\
\hline 10 & 0 & 10 & 10 \\
\hline 10 & 1 & 11 & 11 \\
\hline 11 & 0 & 10 & 10 \\
\hline 11 & 1 & 10 & 11 \\
\hline
\end{tabular}

The "ant trail" problem, as it is often referred to, used a 453 bit allomling? allowing fer a possible 32 states and 4 possible actions fonove forwald, tuan legt. turn right. do nothing) with 2 possible outcomes tood present of 110 fom present).

The genorne that encodes the FSA can be seen in Table 9. The bit strulg cucoutuly this organism consists of the initial state and the last two columms. The furst two columms med 
not be encisded because they are in canonical order. The encoding is highlighted by heavy lines in the above table.

This study is particularly interesting: in that the authors were particularly conscious of possible hias that could be introduced into their experiments by "appropriate" choice of genetic operators. Hence, the crossover operator which was used in the experiments was stindard; being applied blindly to all positions of the bit string without regard to the Ixoundaries of semantic units. The mutation operator was applied equally to all members of the population and mating performed randomly with reproduction according to a stindlard roulette wheel strategy. The fitness function in this work presented itself naturally from the environment itself; namely, the number of pieces of food founc in a bounded number of moves!

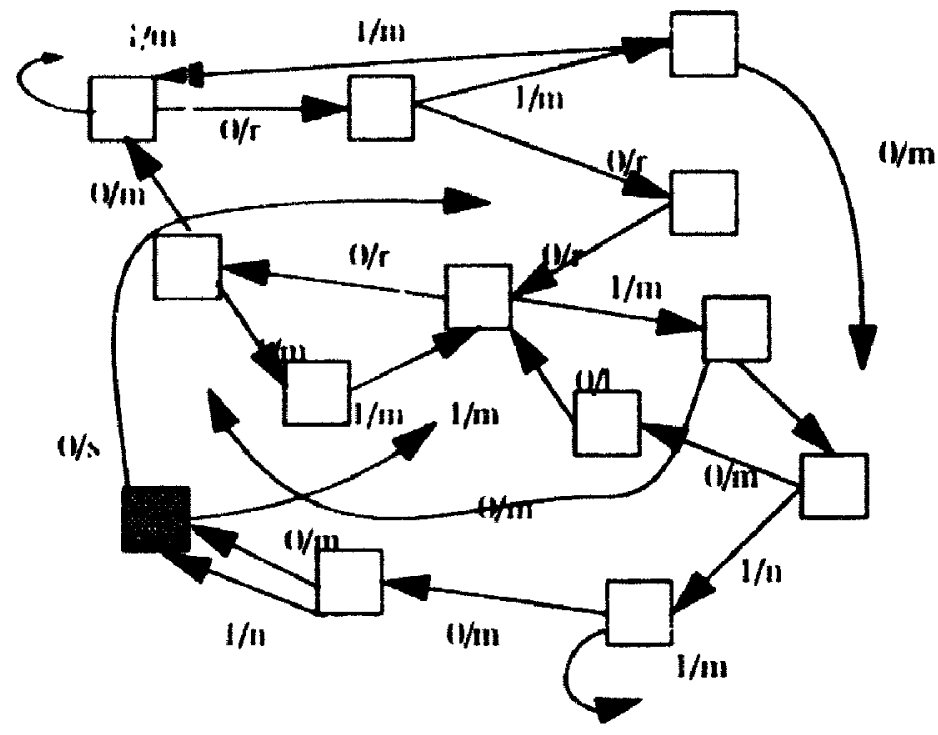

Legend

$L=$ left turn

$\mathbf{R}=$ right turn

M=move

$\mathbf{N}=$ do nothing

$0=$ no food

$1=$ food

=start state

\section{Figure 19 Champion FSA}

Various successful FSA's developed, one of which is shown in Figure 19. The finite state machine is a complex one. and Jefferson et al go to great lengths to demonstrate that such a structure could not have evolved by purely random means. They conclude that

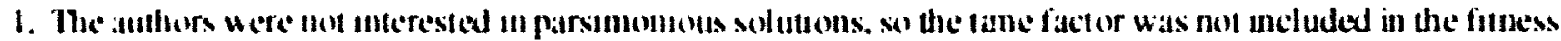

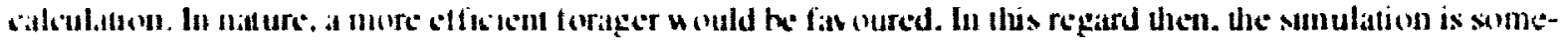
what lunated. 
evolutionary influences caused such an FSA to develop. From an initially ramdinm population of FSAs, with an average ability of $3^{\prime}$ and including a large percentage of totally incompetent foragers. the population evolves to kevels of sophistication ohsersed in

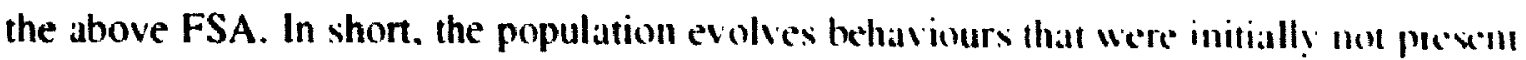
in the population suggesting that "evolution has had the effect of "compiling" hom ledgec of this environment into the structure of the organism."

This led the authors to draw the conclusion that it is computationally foasible an produce artificial organisms that exhbut complex lxhaviour using croluthollary strategies.

More conventional approaches to automated programming have luedl lex forthcoming. This is due - in no small part - to the fragile nature of prongummumy languages themselves. Considering a progrann to be a string af symluls, a single missing piece of punctuation can completely change the meaning of a prognan and probably ensure that it is syntactically incorrect. The usual gentetic operatens of mutation and crossover are dixastrous for traditional programming languages.

However, undeterred, Fujiki |Fujiki and Dickinson. IU87| genemated propuams using a simplificd form of Lisp in order to solve the problem of the Prisomer's I bilemma (see section 3.3.1) and were effective in reproducing Axelrod's work. 'Ihe signiticance at this work is in the application of the genetic algorithen to the gencration of real sultu. code. Fujika and Dickinson's work shows that the genetic algorithm can !x uxed in limd optimal solutions to less than trivial problems when a proper set al proxluctions anc used.

Cramer |Cramer, 1985| used a subset of a simple algorithmic language II. a pseudo-assembly language - in order to prosluce two input, single cutpun multiplication functions. Cramer altempts to preserve potentially uxeful cinte in the population from destruction by the crossover and mutatum eperantow by dividing the program into blocks. These blocks are exchanged as unnts, in lke crossover operation and the mutation operator restricted fo areas of the proplatll which are "non-catastrophic." Unfortumately. the real problem in altemptimy "n

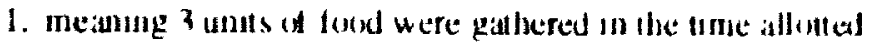


evolve such a program is in the construction of a fitness function. If we consider the space of all powsible programs. the space is largely tlat with a fen peaks scatteled on the landscape. To put it another way, most programs are absolutely usclese or are gibberish! A fitness functuon was developed which gave credit for partial solutions but this was, of course, highly domain dependent. More recently. fllarvey. iqull appears to have resolved the problem of the "stark" landscape by inclentifying "usetul" program elements in a general way, but his descripticns are far from clear to this author.

By fal the most promising application of genetic algorithms to the problem of automatic programming can be attributed to Koza $\mid \mathrm{Koza}, 1990$ ! For a detailed description of genetic programming, the reader is referred to |Koza, 1992|. Programs, in the genetic programming paradigm, at epresented as LISP S-expressions'. The LISP S-expressions are manpulated in their parse tree firmulation using the crossover and mutation operattons which now have ypecial forms.

The crossover operator now takes a subtree from one parent and exchanges it with the subtree rooted at the same losation in the other parent. The mutation operator picks a mode and replaces we subtree rooted at that node with another randomly ferlerated subtrec. An example is shown in Figure 20.
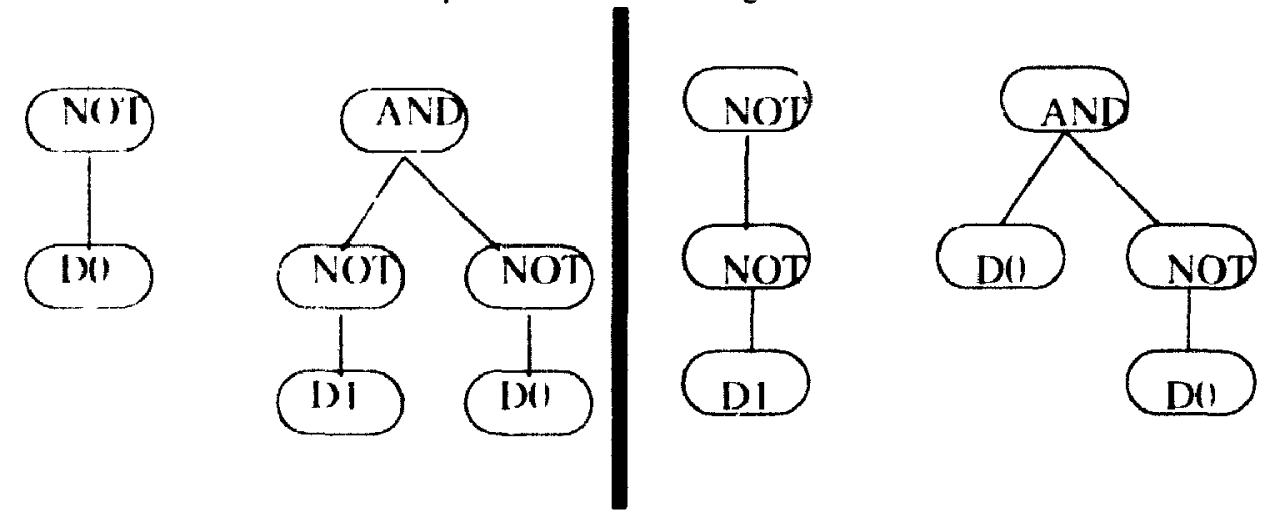

Vijgurs 20 (ienetic Programming ('rossover (Jperation

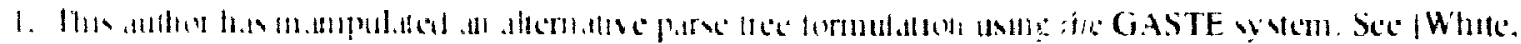
lik: 
Genetic Programming is a rapidly expanding alea of rexilloh mterest and Ku/a has already shown that it can applied in a wide range of program inducthon problems. Programs have been produced genelically for problems in such divers. areas as:

- Induction of decision trees

- mathematical discovery of relationships

- evolution of emergent behaviour

- discovery of game-playing strategics

- planning and

- sequence induction

\subsection{Summary}

The examples described in this chapter represent a cross section of the wouk that has herll undertaken using genetic algorithms. As the previous tive sections have shown, alleas as diverse as function optimization, neural network design. aulomatic programmm! and game playing have seen the successful application of geneluc algorithms.

Traditionally, genetic algorithms in functuon optimisation have locen used in order to investigate the sensitivity of the genetic algorithm lo changes in oproutos design and the parameters which determine their application. This statcey has been continued in this thesis, where function minimization tasks an a valudand test bed of functions has been chosen in order (n demomstralle lle fenclik of combining automata and genetic algorithms.

Game playing was included in order to demonstrate that "programs" with II" notion of strategy could evolve same by the applicatsun of a gemetic atyormlun and, very importantly, could identify hitherto unksown stallegies sppledums of genetic algorithms in the area of automatic programmong demomstanted Harke things. Firstly, that behaviour required fos successful survival condel dievelof under evolutionary pressure. Secondly, that correct generalized solutsons combl develop on the basis of a small number of examples. Finally, that the pertedr algorithm could be successfully applied to an infinite data suacture the pare tree of a program - in order to induce the descrmptions of computer programs. 
The application of genetic search in neural network design has moved quickly from the naive representation where the adjacency matrix representation is directly manipulated by the genetic algorithm to richer representations, such as those proposed by Kitano and Koza. Although genetic search has clearly demomstrated that neural network design can be achieved, scalability remains an isstuc. It remains to be seen as to the prospects of their approaches in real world problems.

Automated programming remains the philosophers' stone of Machine Learning and it is here that genetic al gou ithms have shown considerable promise in the genetic programming paradigm. Ko/a, in his widely influential thesis, has shown that genetic programming can $\mathrm{he}^{2}$ applied to a wide range of program induction tasks - two of which are briefly dexcribed in this chapter. The problem of hierarchical learning - learning to identify program subunits which become part of the set of functions manipulated by the genetic algorithm remaims an open one and we will return to this point later in final chapter of this thesis.

(itnelic algorithms have not been confined to the academic community. They have been widely used in engineering circles as the section on design implies. As such. engineering users have concerned themselves with obtaining optimal solutions where conventional techniques such as hill climbing have failed.

llowever, in all of the above implementations of genetic search. concerns have Ixen ratised as to the art involved in selecting algorithm parameters that allow the algorthm wo ohtain an optimal solution in a reasonable amount of time.

Hence the motivation for this thesis. 


\section{Genetic Algorithm Theory}

The purpose of this chapter is to provide a l... dhematical framework that can le used lo explain why genetic algorithms work. In this chapter the Schema Theorem is deverinped. along with equations that describe schema disruption for two classes of crossovel operator.

\subsection{The Schema Theorem}

The theoretical foundations of genetic algorithms rely on a bit string representatum al solutions. and on the notion of a schema. A schema is a template allowing explonation of similarities amongst chromosomes. A schema can be derived by alugmenting lle domil care symbol (\#) into the alphabet of genes. A schema tepresents all strings - a hyperplant in the search space - which match it on all points other than the \#.

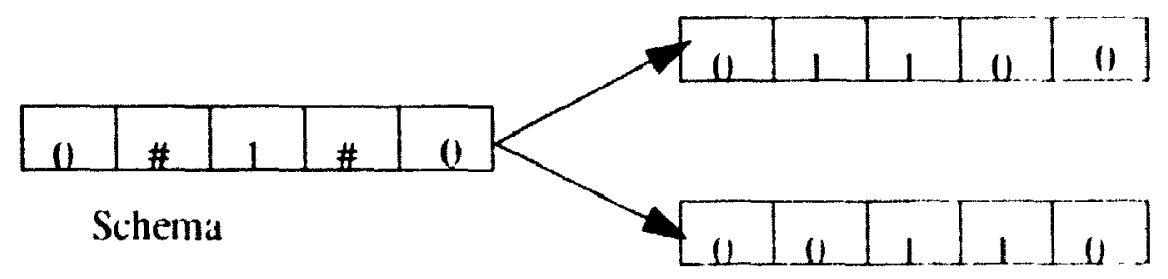

\section{Figure 21 Examples of schema}

Figure 21 shows an example of a shema and the class of strmes whelh it 11 pre "xalls Schema with no \#'s represent only one strong. (ienerally, schema with i \#

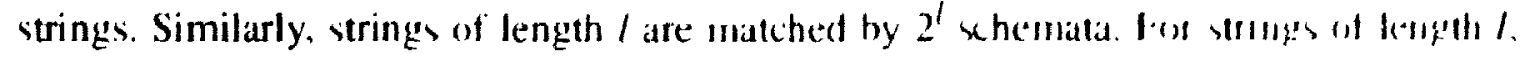


there are $3^{\prime}$ schemata and in a population of size $n$. between $2^{l}$ and $n .2^{l}$ different schemata may be represented.

Different schemata have different properties. There are two important attributes of interest for schema. Firstly, the order of a schema is important. The order of a schema, denoted $o(S)$, is the number of () and 1 positions in the schema i.e. the number of fixed positions in the schema. This measure defines the generality' of the schema. The defining length is al so equal to the length of the string minus the number of don't carc positions in the string. Keferring to Figure 21, we see that the defining length of the example schema is 3 . The number of strings represented by the schema is thus:

$$
n(S)=2^{l-o(S)}
$$

Thus, the lower the order of a schema. the more general the schema and the larger the dimension of the set of strings that the schema represents. The order of a schema is useful in calculating the probability of survival of the schema for mutations.

Secondly, the defining length of a schema is the distance between the first and last fixed string positions i.e. it determines the compactness of the information that is stored in the xhema. Referring once again to Figure 21 , the defining tength of the schema is $\delta(S)=5-1$ $=4$. The larger the defining length of a schema. the less compact the information stored in the schema. The defining length of a schema is required in order to compute the prohahility of survival of a schema during crossover.

As shown in Program (jenctic Algorithm, in Chapter 2. the genetic algorithm can be seen as a process of weition, combination ${ }^{2}$, mutation and evaluation; with the process being reprated until either a number of trials have been performed or a fitness threshold has been reached. If we definc the number of strings matchine a given whema in the population at

1. (1) speriall

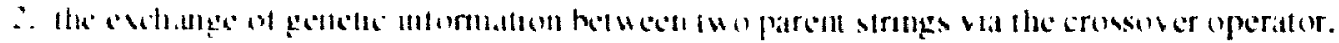


some time $t$ as $m(S . t)$. and tige fitmess of the whema at time $t$ as fis.t). then the wilem. fitness is given by:

$$
F(S, t)=\sum_{i=1}^{b} f_{i}(S, t) / p
$$

where $f_{i}(S, t)$ is the fitness of the ith string in the population which w an ceample of the schema, and there are $p$ such strings in the population at time* $t$.

During the selection stage, an intermediate population of strings is comstrmcked uning fitness proportional reproduction. Each string is copied into the intermediate populatlont with a probability given by:

$$
p_{i}(t)=f_{i}(S, t) / F_{\text {total }}(t) \quad F_{t(t) t a l}(t)=\sum_{i=1}^{n} f_{i}(S, t)
$$

Since for an average string matched by a schema $S$, the probability of its selection is cepual to $F(S, t) / F_{\text {total }}(t)$, and the number of strings matched by a schemal $S$ is m(IS, $\left.t\right)$, the number of strings matching a given schema in the selected population is the'l give'll by:

$$
m(S, t+1)=m(S, t) \cdot n \cdot F(S, t)
$$

or this caun be written:

$$
m(S, t+1)=m(S, t) \cdot F(S, t) / F(t) \quad F(t)=t_{t \prime \prime l a t}(t) / n
$$

Thus the number of strings in the population grows as the ration of the fitmess of seliemat lit the average fitness of the population. Put another way, "above average" schema rene.lve ant increasing number of strings in the next generation while "below average" al hatral Iecerve decreasing numbers of strings in the next generation. 
If we assume that $F(S, t)=(1+\varepsilon) F(t)$, then the growth of schema from one fencration to another can be written as:

$$
m(S, t+1)=m(S, t) \cdot(1+\varepsilon)
$$

which, it we assume $\varepsilon$ remains a constant, is a simple geometric progression and can be solved lo give a closed form for $m(S, t)$.

$$
m(S, t)=m(S .0) \cdot(1+\varepsilon)^{t}
$$

That is, above average schema receive an exponentially increasing number of sirings in the next generation. The above equation is often called the reproductive schema growth equation.

llowever, just reproducing highly fit schema from one generation to another would achicve nothing, as no new sotutions are produced during this process. This is the purpose of the crossover and mutation phases of the genetic algorithm.

Considering single-point crossover only, if a crossover site is selected randomly from anomg the $1-1$ possible sites. the probability that a schema $S$ will be destroyed is given by:

$$
{ }^{\prime}{ }_{d}(S)=\frac{\delta(S)}{l-1}
$$

and that the probabiiity of survival is consequently:

$$
p_{1}(S)=1-\frac{\delta(S)}{1-1}
$$


If the probability of crossover is given by $p_{2, \ldots}$, the probability ot sum

$$
P_{i}(S)=1-P_{1, \ldots} \cdot \begin{aligned}
& \delta(S) \\
& l-1
\end{aligned}
$$

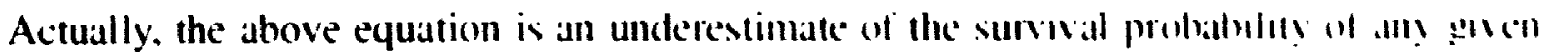
schema. This arises from the fact that even if a crossover vite is selseted henewen tived positions in a whema, there is still a chance that the schema will suvive. Ihis call ckill when the parents exchange genetic information in such a way that the atferded schemb

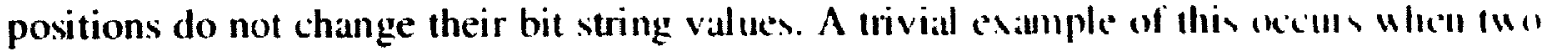
identical parents are mated - crossover does not disrupt any schemal in his ciase. Thi' previous equation is then rewritten:

$$
p_{x}(S) \geq 1-p_{1 \ldots+1} \cdot \begin{aligned}
& \delta(S) \\
& 1-1
\end{aligned}
$$

and the reproductive schema growth equation can be rewritten:

$$
m(S, t+1) \geq m(S, t) \cdot F(S, t) / F(t)\left|\begin{array}{l}
\delta(S) \\
1-l_{1} \ldots \\
1 \ldots 1
\end{array}\right|
$$

The above equation tells us that the expected number of strings matching a scliema $S$ in the next generation still grows exponentially, but that the smaller Ihat schema, the moro likely it is to survive. Hence the justification for the building bleck hyponluess stalled in chapter 2.

The next operator to be considered is mutation. The mutation opetaten andomly chally'esa single position within a chromosome with probability $\mu_{m}$. The etfect is a wegle of a bil from one to zero or vice versa. In order for a schema to survive, all of the hits in the

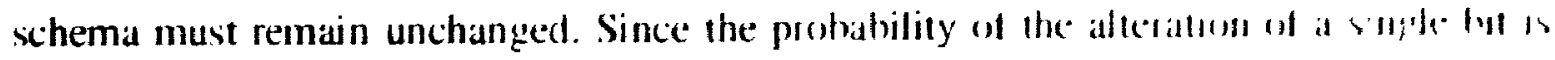


$I_{m}$, the probability of a single bit survival is $1-p_{m}$. A single mutation is independent of any other mutation, so the probability of a schema $S$ surviving a mutation is:

$$
p_{s}(S)=\left(1-p_{m}\right)^{o(S)}
$$

and since $\|_{m} \ll 1$, this probability can be approximated by the first two terms of the binomial expansion:

$$
p_{i}(S)=1-p_{m} \cdot o(S)
$$

and that the reproductive schema growth equation can be rewritten to include the effect of Imutation as:

$$
m(S, t+1) \geq m(S, t) \cdot F(S, t) / \bar{F}(t)\left[1-p_{x o l r} \cdot \frac{\delta(S)}{1-1}-p_{m} \cdot o(S)\right]
$$

This final cquation embodies the Sihema theorem which states:

Theorem I (Schema Theorem). Short, low'-order, above-average schemata recerive cypuncmially increasing wials in subseguent generations of a genetic algorithm.

and a intermediate result of this theorem is that genetic algorithms explore the search space by short. low-order schemata which are used for information exchange during cossurer:

Hypothesis I (Building Block Hypothesis). A genetic algorithm secks near optimal perfirmance through the jutaposition of short. low'-order, high-performance sehemata. ralle'd the lowilding blochs.

This hypothesis suggests that the problem of finding a "good" coding for a genetic al gorrithm is critical for its performance and that related bits should be "close together" in 


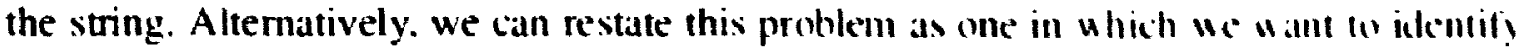
those bits that should always be kept together during crommot. This is a crucial observation in this thesis, as it is exactly the purpose for which adaptive uniform crossover has been designed.

The real value of the genetic algorithm comes from its ability lo procesixes lange numbers of schemata in a single generation. Holland calls this implicit paralledism |llolland. 197.51. Even though $n$ structures are processed in ally one generation, the gemelic allgurilum processes something like $\mathrm{n}^{3}$ schemata. This can be shown as beluu.

Consider a population of $n$ binary strings of length /. If only schemata that surver with . probability greater than $p$ (a constann) are considered. and simple cromover with anly .! small mutation rate are allowed. we admit only those schemata with an corror ralle $r$ I $p_{s}$. Hence, only schemata with a length $l_{s}<\varepsilon(l-1)+1$ arc convidered.

A lower bound on the number of unique schemata processied hy an initially rallom population can be estimated by first counting the number of schematta of length $l_{3}$, or less. This is then multiplied by an appropriate population size such that. on averigle, mo minc than one of each schema of length $l_{s} / 2$.

As shown earlier, the number of schemata of size $I_{s}-1$ in an initial population is given by $2^{(l-1)}$. This calculation can be performed a total of $l-l_{3}-1$ times and that the numles of schemata of length $l_{s}$ or less is $2^{(I,-1)} \cdot\left(l-l_{s}+1\right)$ This is the number ot schemata in a single string. In order to overestimate the numles of whemala in llic population, we just multiply by the number of strings, II. The coumt at the mumlxe of schemata in the population is then given by:

$$
N=2^{(1,-1)} \cdot(1-1+1) \cdot n
$$

This is certainly an overestimate of the number of schemata in the pepulatsont at there will

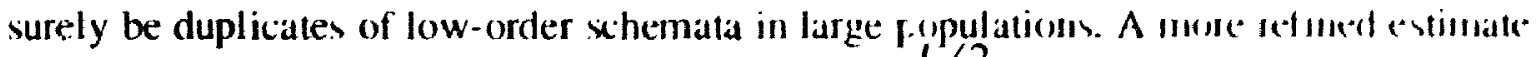
can be obtained by picking a population sia $n=2^{\circ}$. In this way we expect wo have

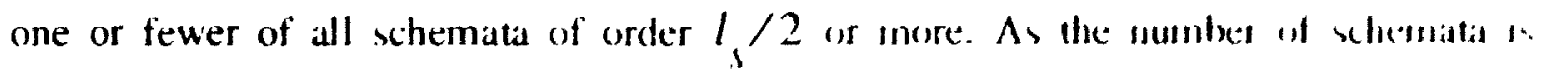


hinomially distributed, half of the xhemata are of higher order than $/ s / 2$ and half are of whaller order. ('ounting only the higher order schemata, a lower bound on the number of xhemata in the population is:

$$
N \geq 2^{(l-2)} \cdot(l-l+1) \cdot n
$$

and that as $n=2^{1 / 2}$. the number of xhemata in the population can be written as:

$$
N=(l-l+1) \cdot n^{3} / 4
$$

i.c. Ihe numlerer of xichemata is proportional to the cube of the population size. Hence. even though the crossover and mutation operators disrupt long, high-order schemata, genetic algorithm implicitly process a large quantity of whemata while processing a relatively whall number of strings.

\subsection{Schema Disruption and Combination}

In a more gencral form, the schema theorem can be written as:

$$
m(S, t+1) \geq m(S . t) \cdot F(S, t) / \bar{F}(t)|1-\zeta|
$$

where $\zeta$ is an error term that captures the damage caused by genetic operators such as crosiserer and mutation. The previous form of the reproductive schema growth equation applied in the case where single point crossover and mutation were applied. In the case where unifom crossover is applied. the survival rate is different.

('onsider a schema $k$. composed of $b_{k}$ bits and with a defining length $\delta(k)$. Let the probability of a bit in a given bit string location being the same in both parents be $P$ ok Note that for a random population. $P_{o k}=0.5$ but as the population converges. this probability will move away from $\left(1.5\right.$ to both 0 and 1 . If $n_{h}$ bits are exchanged betweell a 
parent containing schema $k$ and an another parent containing cescullally ramilom hils. tho schema $k$ could survive in either of the oftispring with probability:

$$
\begin{gathered}
P_{1}\left(n_{h}\right)=P_{a k}^{n_{k}} \\
P_{2}\left(n_{h}\right)=P_{a h}^{\left(b_{h}-n_{k}\right)}
\end{gathered}
$$

and

$$
P_{S}\left(n_{k}\right)=P_{1}\left(n_{k}\right)+i 1-P_{1}\left(n_{k}\right) P_{2}\left(n_{k}\right)
$$

which is independent of the defining length of a whema. It $M(m)$ is the mumlxel of way lu, exphange $m$ bits between two parents, $M(m)$ is given by $I$ - I for sngle pomm crommon and $\left(\begin{array}{l}l \\ i\end{array}\right)$ for the uniform crossover operator.

Schema k can have from zero to all of its bits exchanged cluning c mover. It in hits at ine I bits of the string are exchanged, the number of posvible watys to exchange i bits frum llic schema are:

$$
\left(\begin{array}{l}
m \\
i
\end{array}\right)\left(\begin{array}{l}
l-m \\
b_{h}-i
\end{array}\right)
$$

For each $m$, there are $M(m)$ ways to get there, and there alte $l+1$ pusshlle value wi in Given $P_{S}\left(n_{h}\right)$ and the above equation, it is possible to compute the avcratge sulvival talle

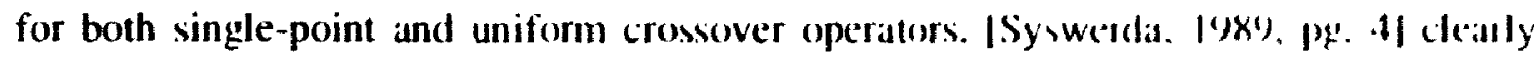
shows that the average survival rate of schema for the uniform operatur is inlerion to that for the one and two-point crossover operators.

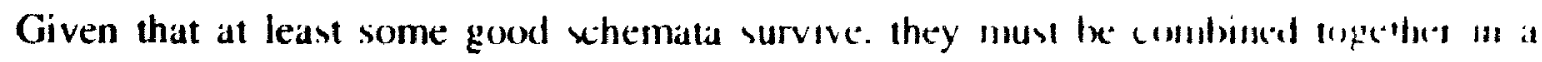
single genome. For the first off spreng to contain good schemata, say $k$ and $1 . k$ mus survive while $j$ is constructed and for the second oft spring, $k$ mast be cemstrue led while 1 
survives. If $P_{,}\left(n_{j}, n_{k}\right)$ is the prohability that schema 1 and $k$ will combine. it is given by:

$$
\begin{aligned}
& P_{1}\left(n_{i} n_{k}\right)=P_{o k}^{n_{i}} P_{o k}^{\left(n_{k}-n_{k}\right)} \\
& P_{2}\left(n_{i} n_{k}\right)=P_{0 k}^{\left(n_{1}-n_{i}\right)} P_{o k}^{n_{k}}
\end{aligned}
$$

and

$$
P_{1}\left(n_{j}, n_{k}\right)=P_{1}\left(n_{j} n_{k}\right)+\left[1-P_{1}\left(n_{j}, n_{k}\right)\right] P_{2}\left(n_{j} n_{k}\right)
$$

As with xhema survival. we must count how many times $P_{C}\left(n_{j} n_{k}\right)$ occurs for all powsible values of $n_{j}$ and $n_{k}$. The number of ways that $m$ bits of $l$ are exchanged, and shema $j$ exchanges $n_{j}$ of its bits, while xhema $k$ exchanges $n_{k}$ of its bits is given by:

$$
\left(\begin{array}{l}
m \\
n_{i}
\end{array}\right)\left(\begin{array}{c}
1-m \\
b_{j}-n_{j}
\end{array}\right)\left(\begin{array}{l}
m \\
n_{k}
\end{array}\right)\left(\begin{array}{c}
1-m \\
b_{k}-n_{k}
\end{array}\right)
$$

As once again. there are $M(m)$ ways of to exchange in bits. the recombination rate can be calculated by counting the above for the possible values of $\mathrm{m}$. $b_{j}$ and $b_{k}$ and using $l^{\prime} .\left({ }_{i}{ }_{h}\right)$ 10 comp'itc a weighted average. [Syswerda. 1989. pg. 5] shows that the uniform crossover operator has a slightly higher average combination rate than either one or iwo-point crossover and atributes the improved performance of the uniform crossover operattor in the empirical studies undertaken to this factor.

(iisen the lach of nepresematiomal bias of the uniform crossover operator, its superior shema rocombination properties. and the studies that indicate improved performance when uvng it in function optimization preblems. it was used as the basis for adaptive oprotor search. This is discussed in depth in Chapier $x$. 


\section{Genetic Algorithms and other Optimization techniques}

\subsection{Overview}

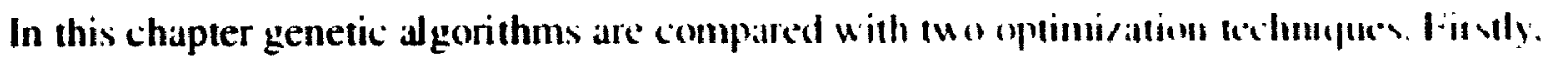
the similarities and differences between evolutionary valegios and ge'lletic alyenilhms alle

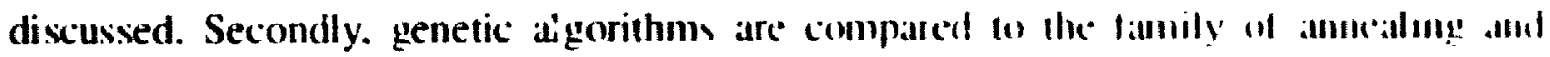
reannealing function optimization techniques; concentrating an the most recent Veny lias Simulated Reannealing method.

\subsection{Evolutionary Sirategies}

Evolutionary strategies (ES) emerged from Rechenberg's work in the late sixlli"s and cally

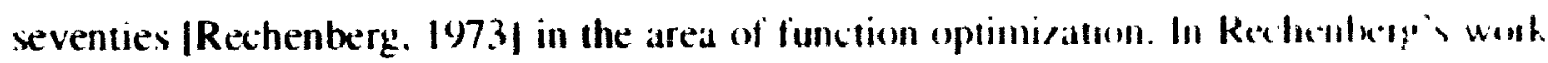

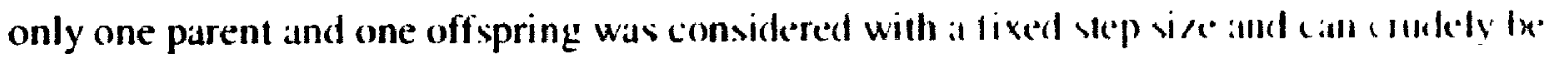
characterized as being a hill climbing algorithm.

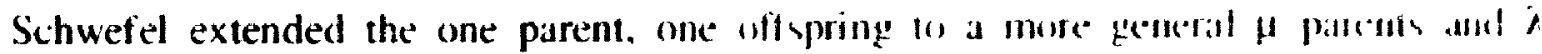
offspring by invoking the biological metapher of evolution. Siliwetel proposed an I:S capable of self adaptation |Schwefel, 1077. 19x/1. An evolutionaty stategy call ix. described using the 11 tuple:

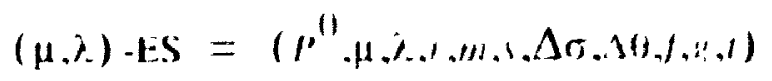




$$
2
$$


where:

\begin{tabular}{|c|c|}
\hline$P^{\prime \prime}=\left(a_{1}^{\prime}, \ldots d_{1}^{\prime \prime}\right)=l^{\prime \prime}$ & Population \\
\hline$\mu=N$ & Number of partuts \\
\hline$\lambda=N: \lambda \geq \mu$ & Number of offspring \\
\hline$r:\left(l^{i} \rightarrow l\right)$ & Recombination operator \\
\hline$m:(l \rightarrow l)$ & Mitation operator \\
\hline$x:\left(t^{\lambda} \rightarrow t^{u}\right)$ & Selection operaltor \\
\hline$\Delta \sigma=\boldsymbol{R}$ & Step size meta contrul \\
\hline$\Delta \theta=R$ & Correlation meta control \\
\hline$f:\left(\boldsymbol{R}^{n} \rightarrow \boldsymbol{K}\right)$ & Objective function \\
\hline$y_{1}:\left(R^{n} \rightarrow R\right)(y \in\{1, \ldots, q\})$ & Constraint functions \\
\hline$t: t^{+2} \rightarrow\{(1,1\}$ & Termination criterion \\
\hline
\end{tabular}

Every individual in the population $a_{1}=\left(x^{\prime}, \theta^{\prime}, \sigma^{\prime}\right) \in P^{\prime}$ consists of thre vecins. The $x^{\circ}$ alle

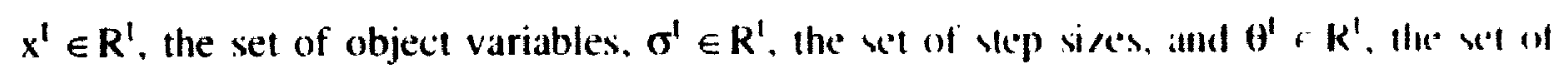
inclination angles. The latter two parameters are internal strategy parametem that contual the mutation of $x^{\prime}$.

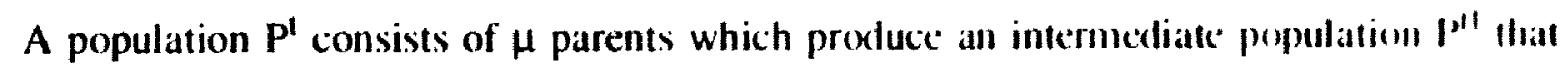

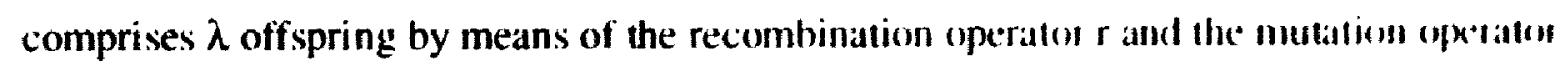
m.

$P^{\prime \prime}=\left(a_{1}^{\prime \prime}, \ldots, u_{n}^{\prime \prime}\right) \in t^{\prime \prime}$

Intermediate Population

$a_{1}^{\prime \prime}=m\left(r\left(P^{r}\right)\right): \forall t \in\{1 \ldots . \lambda\}$

\section{New Population generation}

From this point, evolutionary algorithms can take two forms. Hirstly, in the $(\mu+\lambda) \mid:$ the intermediate population consists of the parents and the off spring - hence $\mu+\lambda . A$ selectiml operator, $s$, is then applied such that the population in the next gentration ss reduced in $\mu$. 


$$
r^{\prime \prime}: 1 r^{\prime \prime}
$$

\section{Culling the Intermediate Population}

The selection operator uses the rule "survival of the fittest individuals" in order to remove less fit individuals from the population. The evolutionary strategy halts when the termination conclition $t\left(P^{\prime}\right)=1$. The individuals of the intermediate population are formed hy the application of the recombination and mutation operators. If $v \in x^{\prime}, \theta^{\prime}, \sigma^{\prime}$ and the recombination operator takes two parents, $a$ and $b$, it combines them according to one of five possibilities:

Table t0 Evolutionary strategy recombination operators

\begin{tabular}{|c|c|}
\hline Result & Meaning \\
\hline$v_{: .1 .1}$ & to recombination \\
\hline$v_{A, 1}$ if $v_{b, 1}$ & discrete \\
\hline $1 / 2\left(v_{., 1}+v_{1,1}\right)$ & Internedialle \\
\hline$v_{, 4,1}$ & ylohal. discrele \\
\hline $1 / 2\left(v_{+4.1}+v_{n_{n, 1}}\right)$ & global intermediale \\
\hline
\end{tabular}

a. This onty inds any meaning for alphathes of size freater that I wo.

In the case of discrete recombination, the vector components are chosen from either parent and $r$ can be viewed as a type of crossover operator with a varying number of crossover points. In the case of global recombination, for each vector component the parents $a_{j}$ and $b_{1}$ are chosen for each position in the vector. This results in a considerably higher mixing of genetic information when compared to the discrete case.

Finally. an offspring is mutated hefore it is evaluated and subsequently placed in the intermediate population. Two types of mutation are defined. Firstly. simple mutations are possible:

$$
\begin{aligned}
& m(a), a \\
& 0 \quad 0 \cdot i v\left(N_{11}(I 0)\right) \\
& i \quad i+N_{1}(A \sigma)
\end{aligned}
$$


where $N_{0}(\Delta \sigma)$ refers to a vector of independent nommally distributed tamdom numlers

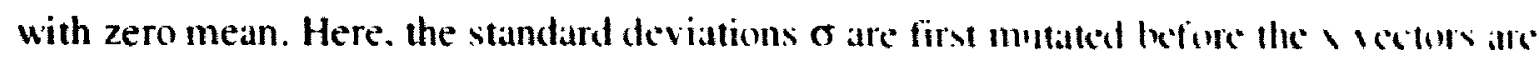
themselves mutated.

Correlated mutations are also possible and were introduced in order Io an aid a pullem with the above mutation mechanism wheren search might Ix- limitcal it: particulat directions as a result of premature convergence.

$$
\begin{aligned}
& m(a)=a " \\
& \sigma^{\circ}=\sigma^{\prime} \cdot(p)\left(N_{0}(\Delta \sigma)\right) \\
& \theta^{\prime \prime}=\theta^{\prime}+N_{11}(\Delta \sigma) \\
& x^{\prime \prime}=x^{\prime}+C_{i t}\left(\sigma^{*}, \theta\right)
\end{aligned}
$$

In the above equations $G_{6}$ refers to a nomally distributed random vectur with meall atro and probability density given by:

$$
\begin{aligned}
& \Delta x=N_{0}(\sigma)
\end{aligned}
$$

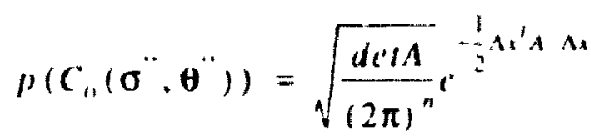

The diagonal elements of the matrix $A^{-1}$ contain the independent variances of the mutation step sizes $\left(\sigma^{2}\right)$ for the object variables $x$. The off-diagonal dements represent the co. variances of the mutations.

The mutation parameter $\theta$ is encoded in the genetic representation of each member of the population and is modified in the same way. In this way, the evolutionary stratcey may adapt to any preferred direction of search by means of self learning.

The strategy of self-adaptation works, as individuals with better parameter values are expected to perform better than the rest. Consequently, election automatically lavours these adapted settings and search speeds up. 
Several of the differences between genetic algorithms and evolutionary strategies arise from the representation chosen a d the quantity of genetic information which is included. Evolutionary strattgies generally deal with real valued strings while genetic algorithms deal most often with bit strings. In short, genetic algorithms operate at the level of the gensilype, whereas evolutionary strategies operate at the phenotypical level. This. however. may just be a consequence of the domain of their applications. Evolutionary strategies have heen used primarily in function optimization. whereas genetic algorithms (as seen in the previous chapter) have been very widely used to solve very diverse problems.

Besides this, the additional vectors $\theta$ and $\sigma$ allow for mutation control to occur individually for each object variable. In genetic algorithms, the mutation rate is usually a global value. Also, allowing $\theta$ and $\sigma$ to be mutated during an algorithm run allows selection to favour better adapted search strategies. In a classical genetic algorithm, adaptation of the search parameters does not occur. It is just this kind of mechanism that precipitated this thesis.

While both classes of search algorithm use principles from natural evolution, such as crossover (or recombination), mutation and selection. the operator design and subsequent dynamic's differ considerably. Evolutionary strategies and genetic al gorithms differ in their use of population. In evolutionary strategies, $\mu$ parents are used to generate $\lambda$ off spring that are added together to form an intermediate population which is $\mu+\lambda$ in size. By maans of selection the $\mu$ best are chosen and from these new parents a new generation is forned by selecting parents two at a time without bias and recombining genetic material. Finally. the offspring are mutated and become part of the next generation. In genetic algorithms the situation is yuite different. Firstly, an intermediate population is generally not created ${ }^{1}$. Parents are again selected two at a time, but the selection process is biased tuwards parents with high fitness. Also. genetic algorithms use crossover (or recounbination) operators which allow for exchange of genetic material contained in the two parents being mated - not globally as in the case of the fourth and fifth operators as

1. Here. We yketh af the classical roukete whed selection process. 


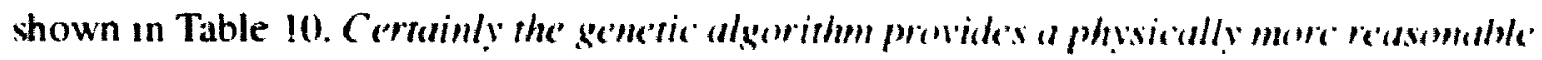

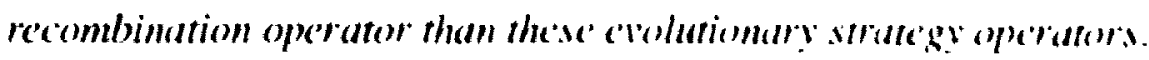

It is possible to argue that the intermediate population fonmed in evolutionary straltegies performs the same task as the roulette theel selection priness of gentic alguritlms. However, it is deterministic, not stochastic. Being stochastic, it is possible for parents with low fitness values to be reproduced into the next generation. With static selection, it is possible to lose valuable genetic inaterial early on with an evolutiomary strate:-y and suftic premature convergence. The ratio $\mu / \lambda$ determines this. In genetic al goratims. premature convergence has also been acknowledged as a problem hut is av oided by judicious chuice of fitness scaling function |DeJong, 19751.

Mutation is used differently in evolutionary strategies and genetic algorithm: In genetic algorithms, mutation is used in order to maintain diversity - lo recover lont alleles it you will. In evolutionary strategies. muation is used in order to perform a hill climbing seallh procedure with the search control parameters being adapted simultancously in oriker lu speed up search. In genetic algorithms, mutation is considered to be a secondary opectator the principal search operator being the crossover operator. This is in complete contrast tu, evolutionary strategies where the mutation operator is considered to in the main starch operator. The standard single (or $n$ ) point crossover operator found in genctic algorithms differs completely from the evolutionary strategy recombination operator. Ilowever, the uniform crossover operator |Syswerda, 1989|- the subject of this thesis' is idcutical to the discrete recombination operator used in evolutionary strategies? ${ }^{2}$ It is interesting to note that the uniform crossover operator has seen increasing favour in genetic algorithm circles since its introduction by Syswerda.

Investigations comparing evolutionary strategies and genetic algorithms have Inen performed for a subset of the function test bed |Hoffmeister, Baick. |W(1). The performance of evolutionary strategies and genetic algorithms were compared for functions $f_{6}$ and $f_{7}$. While the solutions for evolutionary strategies weste superior, it is

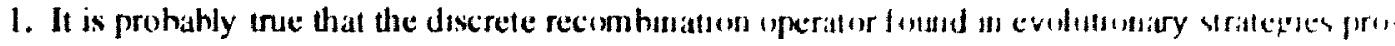
vided that stimulus for this thesis.

2. Assuming that we ignore the cardually of the afphatet. 
unclear whether this statement can be generalized. The importance of parameter selection still appeared to be the deciding factor in algorithm performance.

The most important difference between genetic algorithms and evolutionary strategies from the point of 'ie' w' of this thesis is that the parameters controlling the search process are dynamic in evolutionary strategies but static in genetic algorithm search. It should be noted that evolutionary strategies only implement dynamic parameter setting for the mutation operator.

Chapter $X$ provides details on the adaptive algorithm used for the crossover operator in genetic search.

\subsection{Annealing Algorithms}

Annealing algorithms are based upon ideas taken from the area of Statistical Mechanics in Physics. This class of algorithms rely on their ability to sanple the solution space ergoclically - concentrating trials in regions of high or increasing promise. Three approaches have been described in the literature and are outlined in the following three sections. Annealing algorithms have been applied in a wide range of application areas such as design [Kirkpatrick et al, 19831. finance [Ingber. 1990, 1991a| and neuroscience Ilngher. 1991b, 19921. Firstly, the three approaches will be described; followed by a section which compares genetic algorithms to them.

Comprehensive coverage of simulated annealing algorithms can be found in /Davis. |987|'. |Collins et al. 1988|, |Gelfand. 1987|. |Johnson et al, 1987| and |Laarhoven and Aarts. $19 \times 71$.

The idea of simulated annealing came from the observation that gradient descent methods frequently get trapped in local minima. The minium that is found depends upon the starting conditions for the problem. This can easily be seen in Figure 22 where a solution

1. Also moledes work on gentic algornthms. 
found in valley A - a locul runimum - can never ixe transformed to find the glolval minimum in valley $B$ using gratient descent methods.

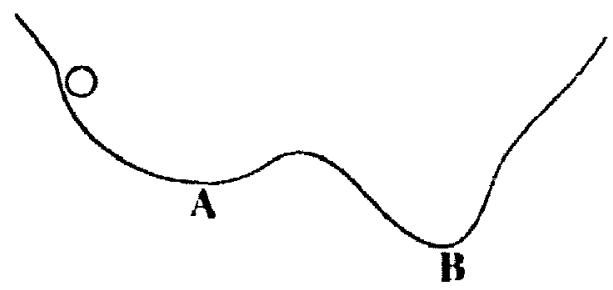

\section{Figure 22 Fitness landscape of a typical function •}

The idea with simulated annealing is to allow occasional uphill sleps! The metaphou is that of annealing in a foundry. In order to find a low energy state of a metal, the metal is heated and then its iemperature is slowly reduced. The problem can le'st $\mid x \cdot$ described by imagining a ball bearing moving about on an energy landscape where the landscape is defined by the function to be minimized.

Referring to Figure 22, if all we can do is descend, we must find ourselves at the hasc al the valley labelled $A$ and so the probability of finding the global minimum depends upon the search start point. In the above figure, as the valleys are of approximately ecyual width the probability of finding the global minimum is above $50 \%$. However, if we comside. a thermodynamic approach where a noise element is added to the solution, the higher lie* temperature of the ball bearing the more violent its thermal motion. Hence, a transition from valley $A$ to valley $B$ is possible and the potential for finding a global, father that a local, minimum exists.

Solution points are generated randomly and their acceptance or rejection is based upul some distribution. In initial investigations, this was a Boltzmann distribution rsee next section). The Bolzmann distribution has the functional form:

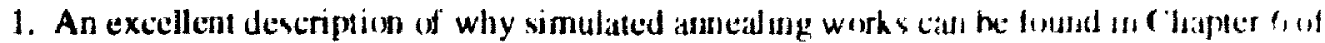
[Rumelhan el id. 1986] 


$$
P_{k}=\frac{1}{\left(1+\exp ^{-\Delta E_{k} / T}\right)}
$$

where $T$ is the "temperature" of the system and $\Delta E_{k}$ is the difference is values of the function being minimized (or the energies of the two states). It can easily be seen that at high temperature, $p_{k}$ is approximately 1 and we have something which allows for almost random search. Only stalles which have a very low energy when compared to the previous statc will be accepted. However, when $T$ is small, the exponential term dominates and be approximately by:

$$
P_{k}=\exp ^{\Delta E_{k} / T}
$$

In this case states with lower energy will be strongly favoured. It is assumed that when the temperature is low, and the exponential term dominates. that the solution is currently exploring the value containing the global minimum.

So, simulating annealing begins with a high temperature and via a temperature reduction $x$-hedule, reduces the volatility of the solution until a desired solution is reached. Simulating annealing can process cost functions of arbitrary degrees of non-linearity. discontinuities and noise and can statistically guarantee finding an optimal solution. The next sections formalizes the above description.

\subsubsection{Boltzmann Annealing}

The technique of Boltzmann annealing (BA) was initially invented in order to assist in the solution of large dimensional integrals that arise in Statistical Physics but was subsequently generalized in order to apply to problems such as finding the optimal wiring for a densely wired computer chip [Kirkpatrick et al, 1983]. The BA process is essentially a Monte Carlo importance sampling technique. 
Considering a problem with D defining parameters, or dimensions, Smulatmy ammealmg̣ can then be characterized with three functional relationships:

1. $g(x)$ : Probability density of state space of D parameters i.e. the way in which whe whlu tion is transformed to the next.

2. $p(x)$ : Probability density for aceeptance of neu cost function value given the gren wus value.

3. $T(k)$ : schedule of "annealing" the "temperature" $T$ in anneal ing timk steps $k, 1.6$.. ot changing the volatility of the two previous probability detnstic?s.

The success of applying simulating annealing to physical problems depends upem the correct choice of the functions $g$ and $h$. Using entropy and minimum frite encrgy arguments [Ingber, 1993], many physical systems can be described using \& of the finm below.

$$
g(x)=(2 \pi T)^{-D / 2}\left(x p\left[\frac{-\Delta t^{2}}{(2 T)}\right]\right.
$$

where $\Delta x$ is the difference between the current test point value and the previous tevt point value and where $T$ is a measure of the fluctuations of the Boltmmann distribution $g$ in ine I) dimensional $x$-space.

The acceptance probability, given a previous state $E_{k}$, is based upon the current state $F_{t+1}$. This is given by:

$$
p(x)=\frac{1}{1+\operatorname{cxp}(\Delta E / T)}
$$

where $\Delta E$ represents the "energy" difference between the present and previous values of the cost function. As [Ingber, 1993] shows, this is just the Boltzmann distribution contributing to the statistical mechanical partition function i in the system. If can be shown [Geman and Geman, 1984 | that a global maximum for $E(x)$ can bx obtained if ' $T$ is selected such that:

$$
T(k)=\frac{T}{\ln (k)}
$$


with $T_{11}$ being "large enough". Although the above equations have been derived on the basis of having physical characteristics, |Kirkpatrick et al, 1983/ demonstrates that the technique applies for problems with reasonable probability densities.

\subsubsection{Fast Anncaling}

Fast annealing (FA) differs from Boltzmann annealing in the area of the generating function $g(x)$. In FA, a Cauchy distribution is used as it has a somewhat fatter tail than the Boltumann distribution. This characteristic assists in allowing easier access to test local minima in the search for a desired global minimum. The Cauchy distribution is shown in the equation below:

$$
g(x)=\frac{T}{\left(\Delta x^{2}+T^{2}\right)^{(D+1) / 2}}
$$

The annealing schedule is al so replaced by:

$$
T(k)=\frac{T_{0}}{k}
$$

\subsubsection{Very Fast Annealing}

In many physical problems we have a D-dimensional parameter space. Different parameters have different ranges and thus different annealing schedules which are determined by the curvature of the cost function at the local minima should be applied to them. The previous annealing algorithms use $g(x)$ distributions which apply across the range of real numbers and are insensitive to individual parameters. Furthermore, fast al gorithms for the generation of a D-dimensional Cauchy random sequence do not exist.

Although the above sequence problem can be avoided by using a D-product of one dimeisional Cauchy sequences. this leads to an annealing schedule given by:

$$
T_{i}(k)=T_{0} / k^{1 / D}
$$


which is faster than the Boltamann annealing shedule but still quite slow. Vity fast simulated reannealing overcomes this problem by using the following:

$$
\begin{gathered}
x_{k}^{i}=x_{k}^{i}+y^{i}\left(B_{i}-A_{i}\right) \\
\left(y^{i} \in|-1.1|\right) ; i_{k}^{i}=\left[A_{i}, B_{i}\right]
\end{gathered}
$$

and defining the generating function:

$$
s_{T}(y)=\prod_{i=1}^{D} \frac{1}{2\left(\left|y^{i}\right|+T_{i}\right) \ln \left(1+T_{i}\right)}
$$

The solution for $y^{i}$ can be shown to be IIngher, 1943]

$$
y^{i}=\operatorname{sgn}\left(u^{i}-\frac{1}{2}\right) T_{i}\left[\left(1+1 / T_{i}\right)^{\left|2 u^{\prime}-1\right|}-1 \mid\right.
$$

where $\mathrm{u}^{\mathrm{i}}$ is a random number from $|0,1|$. The temperature annealing schelule is an exponential function given by:

$$
T_{i}(k)=T_{(1 i} \exp \left(-c_{i} k^{1 / l)}\right)
$$

where $c_{i}$ is given by:

$$
c_{i}=m_{i} \exp \left(-n_{i} l\right)
$$


and $m_{1}, n_{1}$ are VFSK tuning parameters. The acceptance function $h$. is similar!y defined. replacing the Boltzmann fom used in the Boltzmann and Fast annealing algorithms. Fquation 11 clearly shows that VFSR has an exponential temperature annealing schedule which is comviderably faster than either Boltzmann or Fast annealing.

The $1 \mathrm{crm}$ reannealing comes from the fact that periodically the annealing time is rescaled every $f(x)$ or so acceptance events - al though this requires a knowledge of the derivative of the function 10 do this. Reannealing arises naturally out of the changing sensitivities of the $x^{\prime}$ as the search progresses. If we define the sensitivity of the ith parameter as:

$$
\begin{gathered}
x_{i}=\frac{\partial F}{\partial r^{i}} \\
T_{i k}=T_{i k}\left(s_{\text {max }} / s_{i}\right)
\end{gathered}
$$

Ingher has performed a number of experiments to contrast the performance of VFSR with genetic algorithm search for functions $f_{0}$ to $f_{5}$ and they are presented in IIngber, Rosen, 1992I. To summarize the results presented there; VFSR results were marginally better for $f_{1}, f_{1}, f_{3}$, and $f_{4}$. However, as the paper points out, no attempts to optimize the parameters of the genetic algorithm were performed while presumably Ingber tuned the VFSR runs through a specification of the $m_{i}$ and $n_{i}$ parameters.

VFSR and genetic algorithms differ considerably in that VFSR works with a single solution, whereas genetic algorithms manipulate populations of solutions. VFSR, like all annealing algorithms is designed to sample the solution space ergodically; covering the space is a maximally efficient way.

Inlike VHSR. genetic algorithms do not require any knowledge of function derivatives. VFSK also has a hidden tuning parameter, namely the choice of initial temperature. Ammealing algorithms hase be'n shon'n to be extremely schasitive to the choice of 


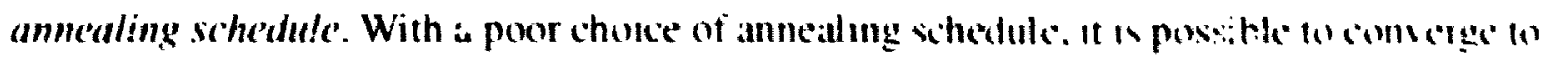
a value that is not the globally optmal value

A fundamental difference hetween simulated annealing and genctle algomthm (N that simulated annealing deals with the problem parameters thenselves - the plathotye

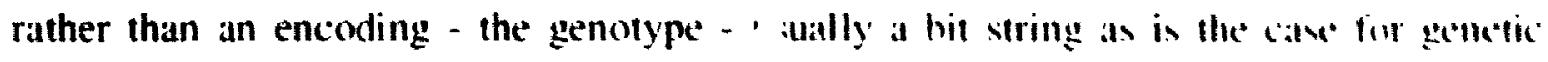
algorithms.

Genetic algorithms also provide a superior ability for paralleliration. as sulpopulatioms can be evaluated independently on separate makhines. While a certain degrece of parallelization is possible for VFSR in cases where the cost function decompones!.

Interestingly, Ackley |Ackley, 1987| has integrated the approaches of vimulatid annealing, hill climbing and genetic algorithms in order to provide all optimmatim technique that improves on each. While (ioldberg $\mid$ (ioldberg. $\mid$ w) $)_{\text {al }} \mid$ has used vimulated annealing on populations and Boltzmann welection in order In improve the genelic algorithm.

Finally, evolutionary strategies and simulated annealing have Iten compared f(irmu. Wuertz. 1990). Their conclusion, for the travelling salesman and other problems, was llat little difference between approaches could be seen. They noted that the difficulties at choosing optimal parameters for both approaches tended to be the most significant fatcur when determining which technique was better.

It can clearly be seen from the above comparisons that genetic algorithms do nol mumblity their control parameters dynamically whereas evolutionary strategies do. II seens reasonable to expect that genetic algorithms might benefit from modification of the it control parameters as the run progresses.

This is the subject of this thesis - the details of the adaptive process are found in (happler $x$

1. The function is of the form $\Gamma \phi_{1}\left(x_{1}\right)$, r $\sum Q_{1}\left(x_{1}\right)$ 


\section{Adaptive Genetic Algorithms}

Chapter 5 described evolutionary strategies and simulated annealing where both classes of algorithm have been extended with an adaptive component. In the case of evolutionary strategies, the step size for the mutation operator is adaptive. while in the case of very fast simulated reannealing. problem variables and the temperature annealing schedule are dynamically rescaled in order to provide improved search. In |Hoffmeister, Baick, 1990|, they observe:

"Self adaptive strategy parameters remain a large blank area on the GA map."

While this is not entirely true, little work has been done in this area. This chapter provides a review of the research that has been performed. It consists of 5 sections.

\subsection{Convergence control}

This section might well be re-titled "diversity maintenance" as this is the problem that convergence control attempts to solve. Premature convergence in genetic algorithms. where one (or possibly several) super individual(s) dominates the population causing premature allele loss, has long been considered a problem. While fitness waling has been considered a partial solution IDeJong. 19751 to this problem. it provides for a static. rather than dynamic solution. Two of the adaptive approaches to convergence control are described below. 


\subsubsection{Ranking and Adaptive Population Size}

The first due to Bake. |Baker. 19851 attempts to monitur rapid comsergentes dus to domination of the population by one of more super individuals ams its descendents. This is achieved using two modifications of the hasic peothetic algorithm. The first modification is to replace the stalladart rouletie wherl selection with a ranking selection method.

In the ranking selection algorithm each individual receiven an expected number of offspring based upon the rank of the bit strings performam and not on its absolute value. The allocation function is monotonic and linear; defined as in the table helou:

Table 11 Ranking allocation function

\begin{tabular}{|l|l|l|}
\hline \multicolumn{1}{|c|}{ Variable } & \multicolumn{1}{|c|}{ Meaning } & \multicolumn{1}{c|}{ Formula } \\
\hline MIN & Lower bound & $2.0-\mathrm{MAX}$ \\
\hline INC & Difference between adjacent individuals & $2.0^{\circ}(\mathrm{MAX} \cdot 10) / \mathrm{POP}$ \\
\hline LOW & Lowest individual's expected value & INC / 2.0 \\
\hline POP & Population size & input parameter \\
\hline MAX & Upper bound tor expected values & input parameter \\
\hline
\end{tabular}

Ranking just sorts the population based upon the fitness values of the individuals followed by calculation of the ranking allocation function. The allocalion function is then used to compute the number of offspring of a given parcmt in the next generation. In Baker's function: the expected number of offspring for the fittest individual was determined by the setting of the MAX variable which, through experimentation was set at $1.1^{\prime}$. Although not dynamic, this severely limits the contribution of any given individual and therehy reducess allele loss.

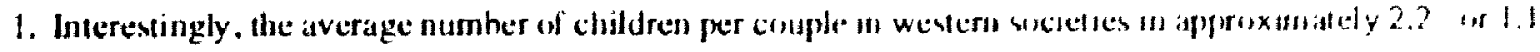
per individuat. 
Michalewicz |Michalewicz, 1992| proposes the use of a non-linear functior:

$$
\operatorname{proh}(r a n k)=q(1-q)^{r a n k-1}
$$

where rumk = 1 corresponds to the best individual, and ramk= population size represents the worst individual. The variable $q$ is a user defined parameter.

The ranking selection method was coupled with a dynamic population size mechanism. Here, Baker defines a percent involvement measure as:

"We define percent involvement as the percentage of the current population which contributes offspring for the next generation."

Individuals are added to the population until both the original population size and a user-defined percentage involvement value is reached. This ensures that during periods of slow convergence the population is constrained to be close to the original population size, as all individuals will be equally involved. However, during periods of rapid convergence - when the percentage involvement will tend to decrease - the population will grow. The population will subsequently shrink back towards the initial population size once convergence slows once more.

This mechanism thus allows for dynamic modification of a parameter (population size) through the monitoring of the percentage involvement measure. It is, however, still possible for a super individual to make up a large proportion of the population quickly even though allele loss is not seen.

The ranking and dynamic population strategy was used in the study of Shekel's foxholes function $\left(f_{5}\right)$ and was shown to provide superior control of convergence - average bias values being considerably reduced. 


\subsubsection{Diversity Maintenance using Entropy}

Population convergence call also be controlled by monituring the degrec if

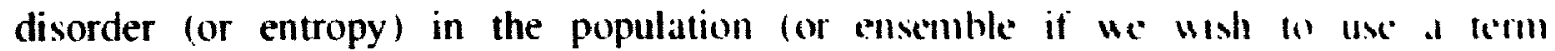
drawn from Thermodynamics.) The diversity of a population can be measured by considering the entropy of the system. This approach, dut to | White, 14021 , combines all elitist. selection strategy with a dy namic mutation operator probahility in order fo maintan population diversity 1 .

The principal concern in this study was the premature convergence often observed in elitist strategy selection genetic algorithms. The elitist strategy consures that the Ix-vt solution will never be lost, while the adaptive mutation operator ensures that sulficient diversity will be maintained.

Considering only a binary alphabet here, and using the Shannon |Shammon. |M-4X| information-theoretic measure, the entropy of a population can lx stated as:

$$
\begin{gathered}
E(\bar{p})=-\left(\sum_{i=1}^{l} p_{1}^{i} \ln p_{1}^{i}+\sum_{i=1}^{l} p_{0}^{i} \ln p_{0}^{i}\right) \\
p_{1}^{i}=\left(\sum_{j=1}^{P_{s} \operatorname{bit}(i, j)}\right) / p_{s} ; p_{0}^{i}=1-p_{1}^{i}
\end{gathered}
$$

where $I$ is the length of the bit string, and $P_{s}$ is the size of the population. It cant cansily Ix* shown that this function is maximized with:

$$
p_{1}^{i}=p_{0}^{i}=\frac{1}{2} ; i=(1, \ldots, l)
$$

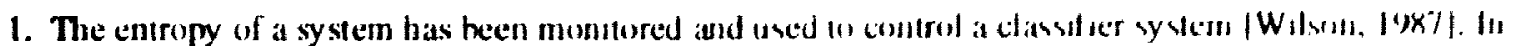

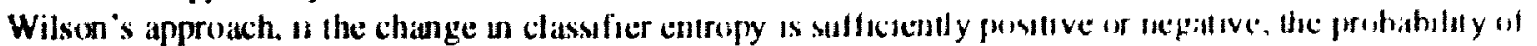

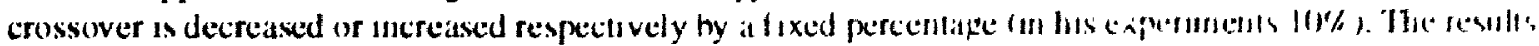

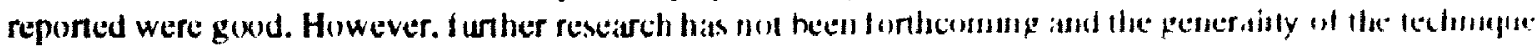
is in question. 
Det ining $E_{\text {max }}$ to be:

$$
E_{\text {max }}=E\left(\overline{p_{\text {max }}}\right)=l \cdot \ln 2
$$

the diversity of the population of bit strings can be defined:

$$
D=\frac{E(\bar{p})}{E_{\max }}
$$

A plot of the diversity function in one dimension is shown in Figure 23.

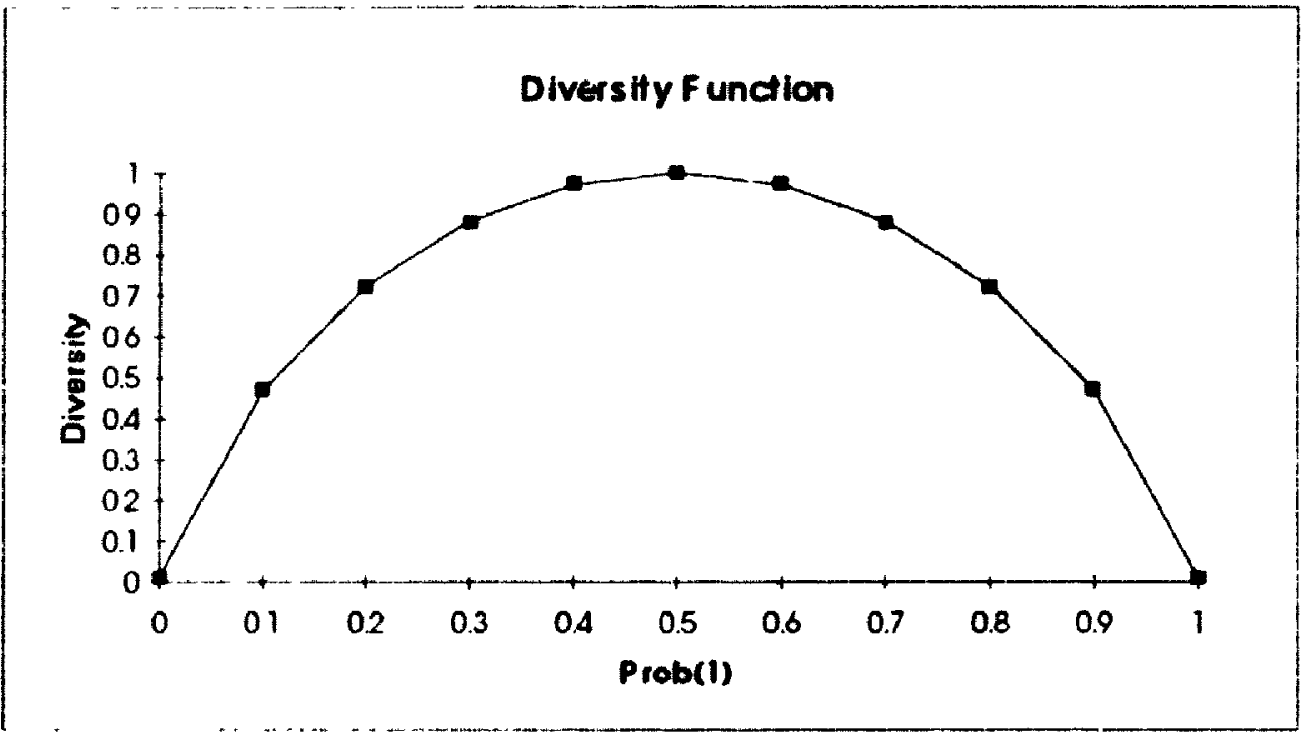

Figure 2.3 Hot of the Diversity Function

The diversity function is a function that is convex, symmetric about 0.5 , and lies in the range $(0,1)$. The generational value of the mutation operator probability is generally defined to be:

$$
p_{m}=g(D)
$$


where $g(D)$ was defined to be:

$$
g(D)=p_{\max }-D\left(p_{\max }-p_{\min }\right)
$$

$p_{\max }$ was constrained to be in $(0,0.1)$ and $p_{m m}$ to be less than P $p_{m . x}$. The parameters P'mum and $p_{m a x}{ }^{\prime}$ are algorithm parameters. With $p_{m}$ approximately equal to 0.5 (i.c. when there is little diversity in the population) the genetic algorithm reduces to hill climhing search.

T.ee dynamic mutation operator was applied to function $f_{1}$ and foums to Ix. extremely effective at preventing premature convergence, and allik loss. However, it was felt that the mutation rates were gencrally low high for reasonable diversity values and it was proposed that a non-linear $g(D)$ function the used. An alternative function proposed ${ }^{2}$, but not investigated, was:

$$
g(D)=P_{\max } \exp (-D k)
$$

where $k$ is constant such that:

$$
k=-\ln \left(\frac{P_{\text {minl }}}{P_{\text {max }}}\right)
$$

By defining $k$ in this way the function $g(D)$ is constrained to lie in the range $\left(p_{\min }, p_{\max }\right)$. It should oe noted that the functional form of this equation draws its inspiration from the temperature annealing schedule used in simulated anmealing. A plot

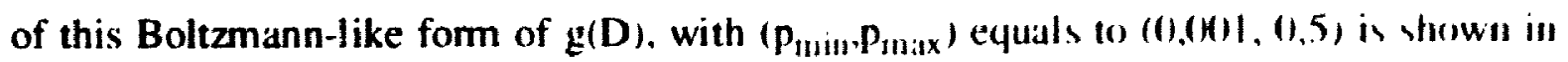
Figure 24.

1. $p_{\text {max }} . p_{\min }$ values were $0.1,(1,(1)$ in the study pert ormed.

2. This is smilar to [Fogany. 1989] 


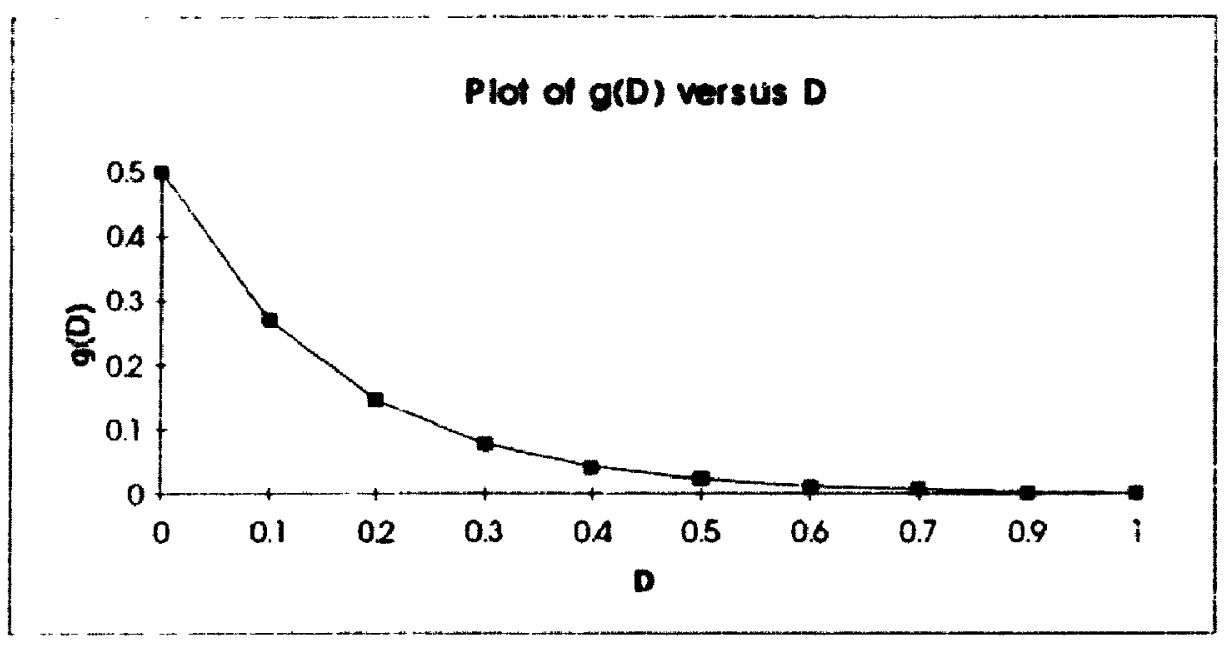

\section{Figure 24 Mutation Probability as a Function of Diversity}

From the form of this curve it can be seen that at high diversity - as in the initial randomlygenerated population - the mutation probability is low.

\section{Plot of $\mathrm{fl}$ Diversity}

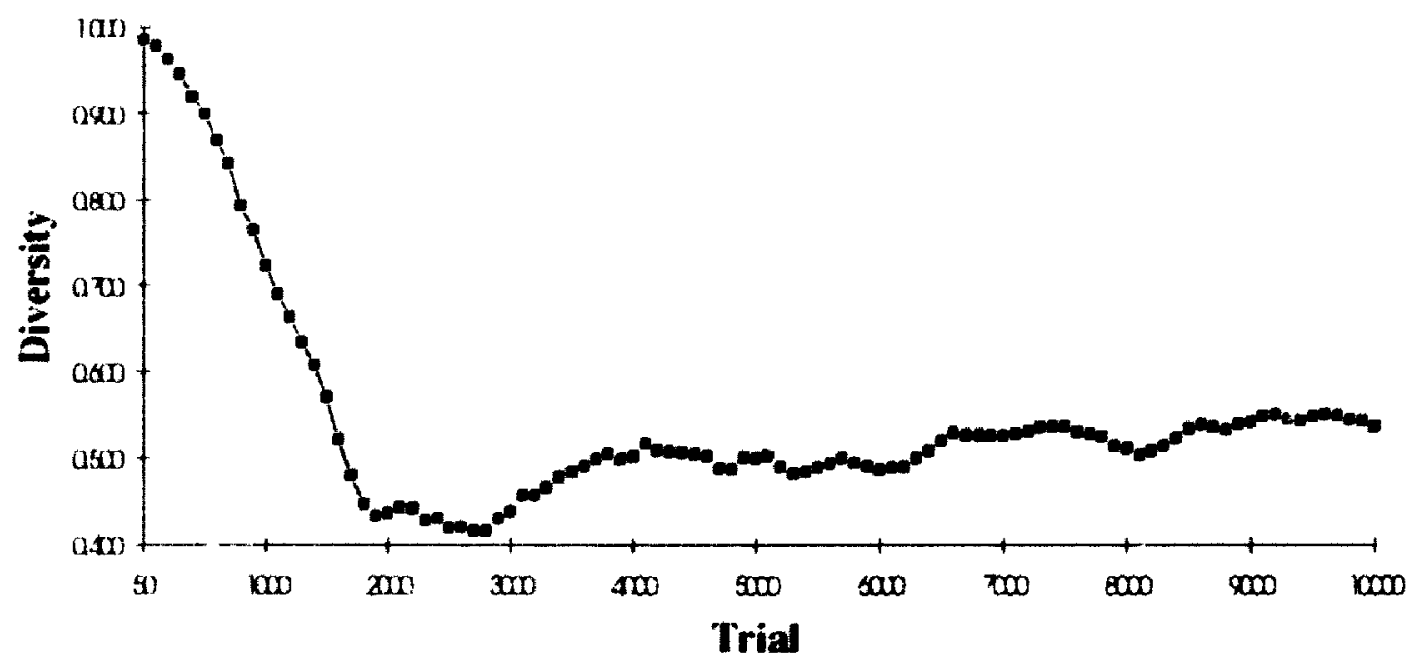

Figure 25 Diversity as a function of trial for $\mathbf{f l}$ 
However, as selection pressure gradtally reduces the diversity of the overall population. the mutation probability rises slowly but with an ever increasing rate to its mavimum value at $D=1$ ). A plot of the diversity seen in the population for function $f 1$ ass a function of trial can be seen in Figure 25.

The speed with which this function falls off is strongly influenced by the ratio $p_{m u} / n_{m a n}$. A criticism that can be leveled against the above adaption mechanism is that it requires the calculation of a global measure - population diversity. Genetic algorithm purists' ${ }^{1}$ tend not to use mechanisms that require such calculations.

\subsection{Punctuated Crossover}

Traditional i-point crossover is known to be biased in the sellse that hits at the ends of the string are not manipulated with the same efficiency as those in other locations on the string and the success of the operator depends strongly all the representation chosen - the further apart "linked" genes are, the more likely they are to be split apart by the action of the crossover operator. In somk sense, traditional 1-point crossover can be said to be "blind": caring not for the allele at a particular location. This is in complete contrast to Nature's genetic crossover activity which strongly indicates that the location of crossover events depends upon the contents of the cisromosomes at that location. Schaffer |Sichatfer, 11987| identified this and it provided the motivation for the promstuancd crossover operator. The idea of punctuation in strings was first documented in Illolland. 1975] but in Schaffer's work was used in quite a different way.

The punctuated crossover operator requires the doubling of the string length, where the first 1 bits are the conventional representation and the second I bits are imterpreked as crossover punctation. A one in a particular bit position means that croswiver is allowed, whereas a zero in any given bit position implies that crossover at that location is

1. Purists in the sense of altempting to retion as much of the genetic metaphior as prosstib: 
prohibited. An alternative view of this representation is to think of it as a diploid structure. An example of this diploid view can be seen in Figure 26.

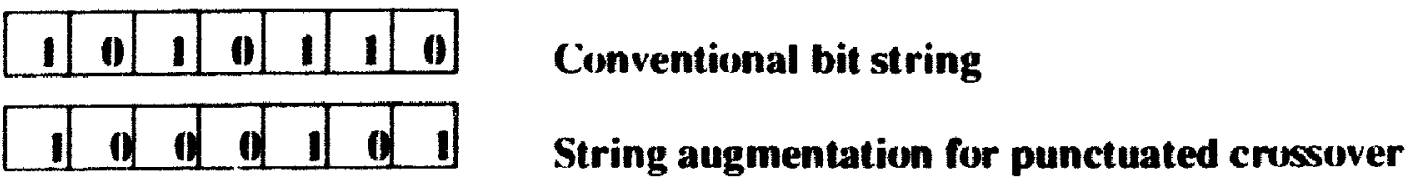

Figure 26 Punctuated crussuver bit string representation

While the initial population conventional bit string components were generated randomly, the augmented parts were governed by $P_{x o}$, the probability that a crossover was allowed at a particular location. The punctuated crossover operator works by exchanging bits according to the punctuation in both parents. When a 1 bit is encountered in either parent, the bits begin going to the other offspring i.e. the bits are crossed over. When this occurs, the punctuation bits are also exchanged. The punctuation bits are considered to be linked to the conventional bit in the overall representation.

Thus. certain distributions of parental bits will result in all of the punctuation bits being passed to one of the offspring and none to its sibling, while others will tend to distributa groups of them. As a result of standard selection techniques. when an offspring fails to survive to the next generation. its punctuation bits die with it. Hence. the dynamics of punctuation bit distributions reflect an accumulating experience about where good locations for crossover are to be found.

As such, the mutation operator acts on both the conventional bit string and the punctuation hits. Schaffer indicates that this mechanism generates for superior results but provides no experimental evidence to support this view.

Schaffer provides results for the application of the punctuated crossover operator on functions $f_{1}$ to $f_{5}$ : contrasting them with Grefenstette's results for the same 
functions derived using a meta-GA /Grefenstette, |986). His results are significantly! better in the case of functions $f_{1}, f_{2}, f_{4}, f_{5}$ and no worse for $f_{3}$.

These results, and the idea of an extended representation uned (1) guide the crossover operator, are particularly important to the development of this thess. Clearly, from Schaffer's work. adaptive crossover can play a significant part in the improvement of genetic search.

\subsection{Meta-level Genetic Algorithms}

Grefenstette's work |Grefenstette. 1986/ on meta level genetic algorithms was first suggested by work done by Weinberg [Weinberg, 1970)] where the results of a genetic algorithm run were passed to a higher level genetic algorithm (meta-(iA) as shown in Figure 27.

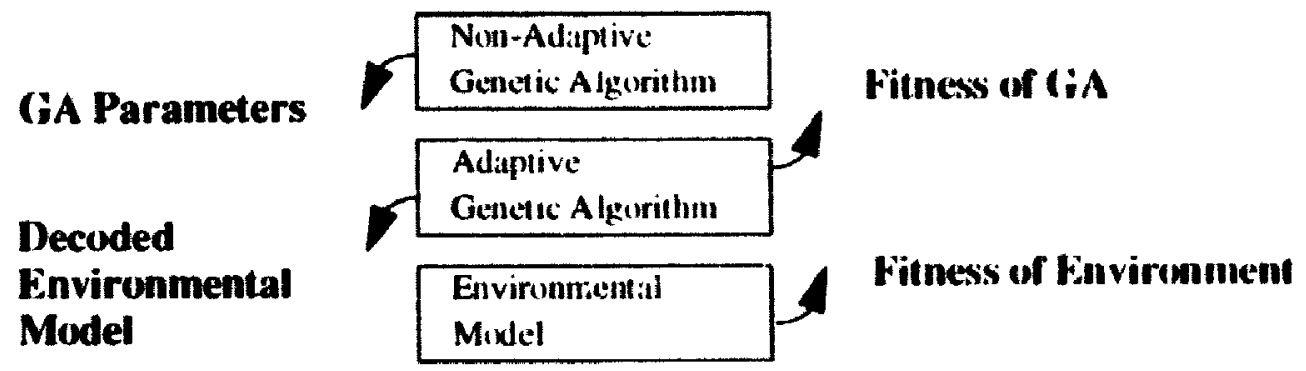

\section{Figure 27 Meta (jenetic Algurithm Architecture}

Grefenstette used the non-adaptive genetic algorithm in oriler 10 allowle parameters for the adaptive genetic algorithm- such as probability of mutation, probability of crossover, and population size. The fitness of the hit strings in the meta-level genetic algorithm were computed by running a genetic alforithm with those parameters for a given function - one of functions $f_{1}$ to $f_{5}$ were used.

This was an extremely expensive process, reyuiring a single genetic algorithm run in order to compute the fitness of a string at the meta-lovel. While infeasible

1. statisucally significant. 
as a texhnique for general function optimization, the experiment did yield results for functions $f_{1}$ io $f_{q}$ which were better than those previously reported.

(irefenstette's new settings have become a default standard for researchers using hiriary representation, thus saving researchers considerable time in deriving parameter setting appropriate to their domains.

Schaffer |Schaffer et al, 1989| subsequently extended Grefenstette's work to include functions $f_{6}, f_{7}$ and three problems drawn from the areas of filter design. a 30 city travelling salesman problem and a graph partitioning problem.

\subsection{Adaptive Operator Selection}

In the traditional genetic algorithm, the number of operators that can manipulate the bit string is fixed, and the probability with which they can be applied is constant. Further, it is also the case that the operators are applied in a given sequence. It was Davis' [Davis, 1989] observation that different operators are generally required at different stages of the search process and that the genetic al gorithm should adapt and be able to select which operators amongst those available to apply and in what order' ${ }^{1}$. Davis worked with a set of five operators, in a non-binary string representation. The operators used are briefly described in the next five paragraphs.

The (iUARANTEED-UNIFORM-CROSSOVER operator creates a child from two parents by deciding on a field-by-field basis which child receives the corresponding value from which parent ${ }^{2}$.

The (GUARANTEED-AVERA(iE operator creates a child by averaging the fields of the two parents. Randomly rounding up or down in the case of an intermediate value?.

1. That is from generillun wo genkeralton.

2. I argely caunsalent to the unif onn crossover operator [Syswerda, 1989].

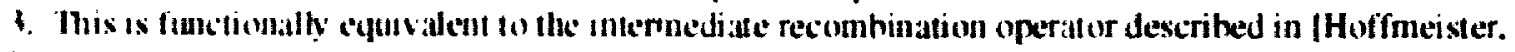

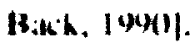


The (IUARANTEED-MUTAMION operator creates a child from ane parem by passing down the parent's fields and. with $10 \%$; probability replace atch value Iy a random value between $(1$ and the maximum allowed for the variable.

The GUARANTEFD-HIG-CREFP operator creates one child from anc farcul by passing down the parent's fields and. with $20 \%$ probatility, altering a lield by replacing it with a value that is 1.2 or 3 units above or below the paremis value.

The (AUARANTEED-LITILE-CRERP operator creates me chill from mil parent by passing down the parent's fields and, wilh $10 \%$ probahility, allaring a field by replacing it with a value that is I unit above or leelow the partent fielal value.

In all of the above operators, a child uas retaimed only if it differed from its parrems.

The idea is that adaptation of cperator probabilities takes place based upwit the performance of the operator's offspring. In short. if the operatur proshecs offspring with a fitness which is superior to that of the parents, then the fitmess af the operator should increase. However, if the operator produces alfspiaig will a fitness which is inferior to that of the parents, then the fitmess of tha! nperalou should decrease. Davis further believed that:

“...an adaptive mechanism should alter the probahility of applying an operator in proportion to the observerl performance of the individuals created by that operator in the course of the run."

and that:

“. purely local measures are not sufficient. (me must reward an operator that sets the stage for this production."

This is often referred to as a credit assignment problem and is commont in Machine Learning problems. To paraphrase the above quote, a window must lo* maintained over which reward-penalty actions are to be performed. An ofx-rator bids to be the one selected and at the end of the window, these bids alc allecalted 
(1) the operators that have caused increasingly fit offspring is proportion to their fituess increase.

The adlaptive algorithm used by Davis was:

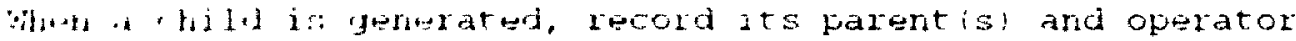

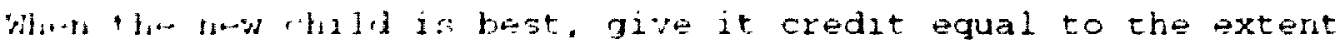

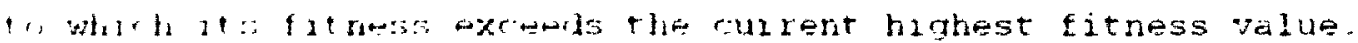

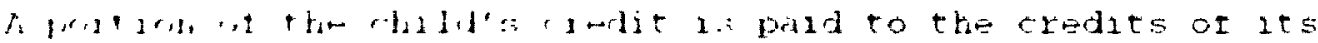

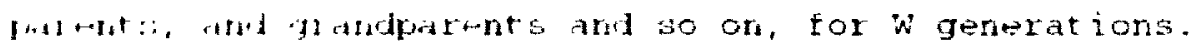

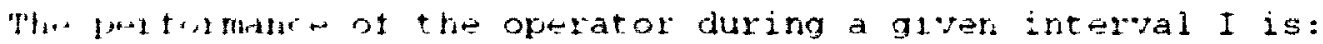

$$
\begin{aligned}
& \text { perf }_{\text {operator }}=\frac{\sum \text { credit }_{\text {children }}}{\text { numberOfC } \text { hildren }}
\end{aligned}
$$

The adaptation of operator fitness at the end of the update period is calculated using the following algorithm:

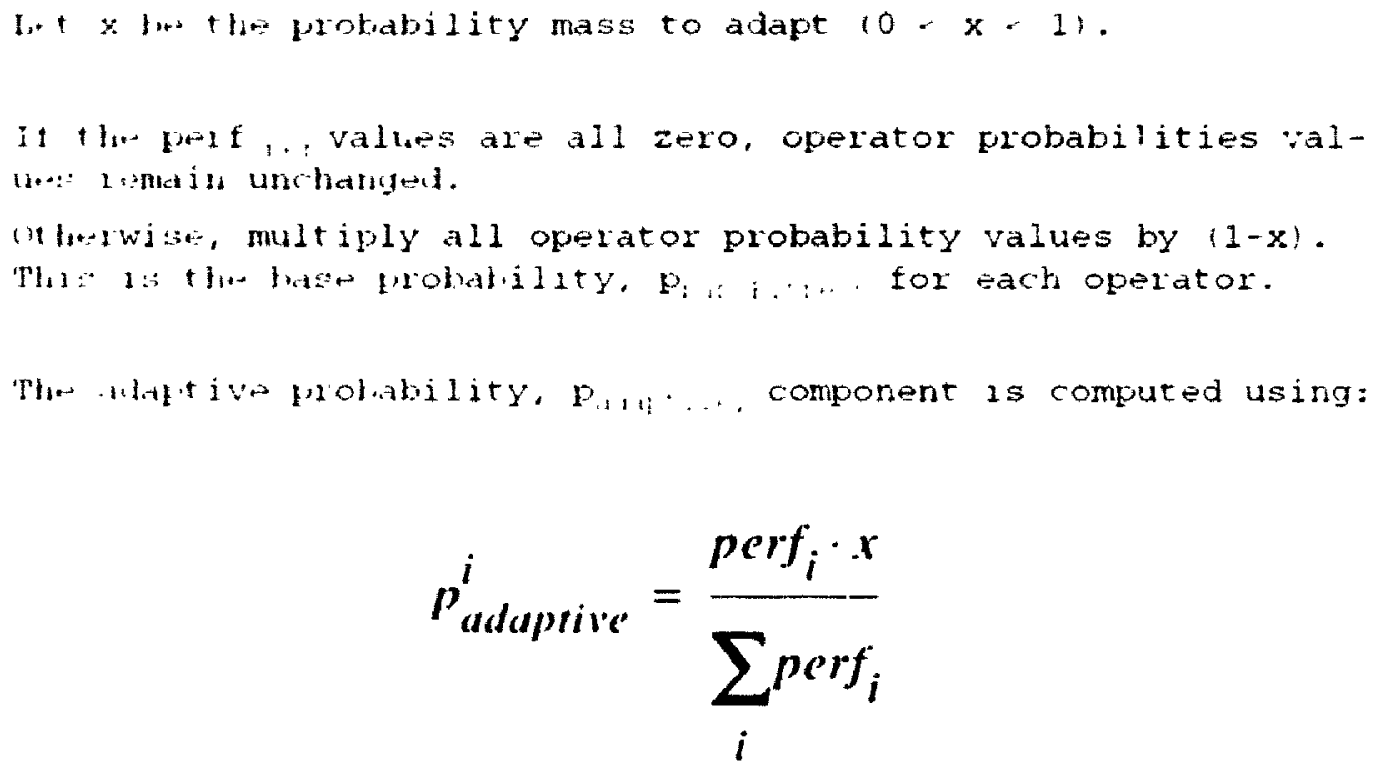$$
p_{\text {adaprive }}^{i}=\frac{\operatorname{perf}_{i} \cdot x}{\sum_{i} p{ }^{\prime} f_{i}}
$$

and that : 


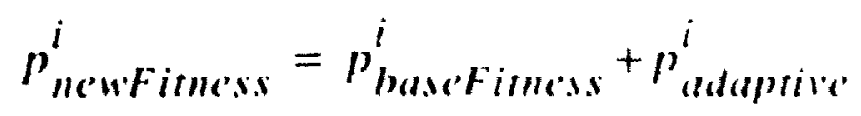

The pnewfitness values are then used as operator probabilities for the nex follitic algorithm interval (I). In Davis" work, the window of adaptation. W. Was iet $10\left|x^{2}\right|(1)$ allit the algorith. - adaptation interval to be 50 . The value of $x$ was set to he 11.15 i.e $1.5 \%$ : 1 the probability mass was reallocated during a single adaptation.

Davis" method provides a general method for having a genetic algorithm chouse which operators to apply for itself. Theoretically, it should |xe possible to sced the problem with a set of candidate operators and have the algorithm decide which to apply as the algorithm progresses - some operators eventually heing discarded.

Although Davis" approach provides a very genceral approach for antaplive operator probabilities based upon the quality of the offypring that they produce. the technique has the significant drawback of having to resolve the credit assignmem problem i.e. the apportionment of credit to an operator that set the stage for the purkluc liull of a high calibre offspring. This is a common problem in Machine I carming.

\subsection{Adaptive Mutation}

Globally mainipulated mutation operator probabilities have been proposed. In |ll|cser. Männer. 19911. an exponentially decaying mutation operator probability is used to contiol search. The form of the operator probability draws its inspiration from concepts found in simulated annealing and evolutionary strategies and is given by the equation below:

$$
P_{m}(t)=\sqrt{\frac{\alpha}{\beta}} \cdot \frac{\exp \left(-\gamma_{2}^{t}\right)}{\lambda \cdot l i}
$$

Here, $\alpha, \beta$ and $\gamma$ are search dependent parameters, $\lambda$ is the population sse and $/$ the length

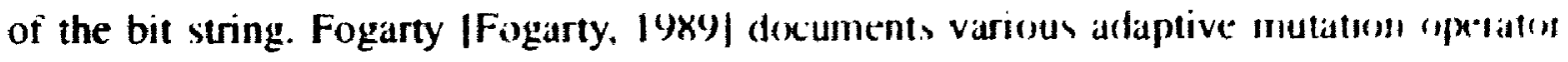


strategies applied to the problem of opumization of parameters used in the control of industrial burner valves. The most promising strategy is that of an initial mutation probability which decays exponentially with each succeeding generation - again hinting at the annealing schedule for temperature found in simulated annealing search algorithms.

Häck in |Bäck, 1991| introduces an adaptive mutation operator which encodes mutation operator probabilities directly into the genetic representation of the individuals. Further. mutation rates are al so subject to mutation and selection in that they undergo evolution as well as the object variables. Unlike Hesser and Männer, no global control of mutation rate adaption is required. Initial mutation rates are set randomly.

As a consequence of this approach, the mutation operator probability is no longer an external parameter of the genetic algorithm. Each bit position now has an asscxiated mutation probability, initially set randomly. The bits encoding the mutation probability represent a number between 11 and 0.5 . The mutation incchanism works by first mutating the mutation rates $p_{i}$ with mutation probabilities $p_{i}$ and then using the mutated probabilities $p_{i}{ }^{\prime}$ to mutate the ohject variables themselves.

In this way, the mutation rates private to particular variables are subject to mutation and therefore change probabilistically over time. This process is directed by the selection process. which selects good object variable information as appropriate stratrgy parameter settings.

Baick used this mechanism in order to study the functions $f_{1}, f_{7}$ and $f_{15}$ and obtained cemarkably good results - considerably better than through non-adaptive means. A schematic of the adaptive mechanism is shown is Figure 28 . 

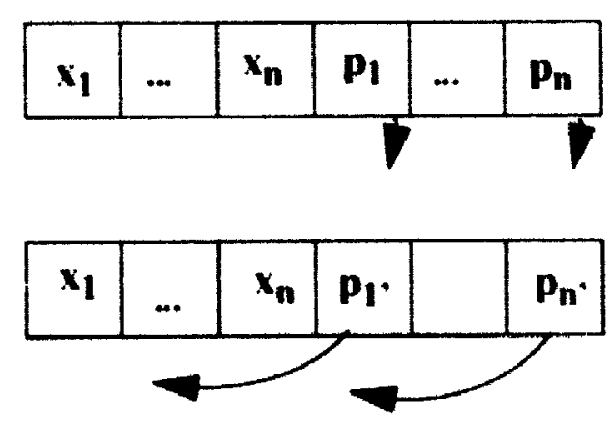

\section{Mutation of inutation rates}

\section{Mutation of object variables}

\section{Figure 28 :Schematic of mechanism used for adaptive mutation}

Bäck maintains that the self-adaptation mechanism works hy an interaction of sufficionl genetic diversity within the strategy parameters and an implicil linh |x.tweon advantageous settings of strategy parameters and good furction values, therehy providing selective pressure towards optimal operator parameter values.

This adaptive operator is relevant to this thesis for two reasons. Firslly, Ilke representation is extended in order to encode mutation operator probability in a per-bit basis. Secondly. the representation ats on itself in orker Io change Ihe values of the operator probabilities themselves. 


\section{Automata Theory}

I cearning automata have been used in the literature to model biological learning systems and also to determine the optimal action an environment offers. The learning is achieved by the automaton interacting with the environment and processing its responses to the actions that are chosen. Automata have seen use in applications such as parameter optimization. statistical decision making and telephone routing [Lakshmivarahan. 1981], |Narendra and Thathachar. 19741, INarendra and Thathachar. 198()|, |Narendra et al., 1977|. The latter paper provides an excellent survey of automata, as does the book by Lakshmivarahan.

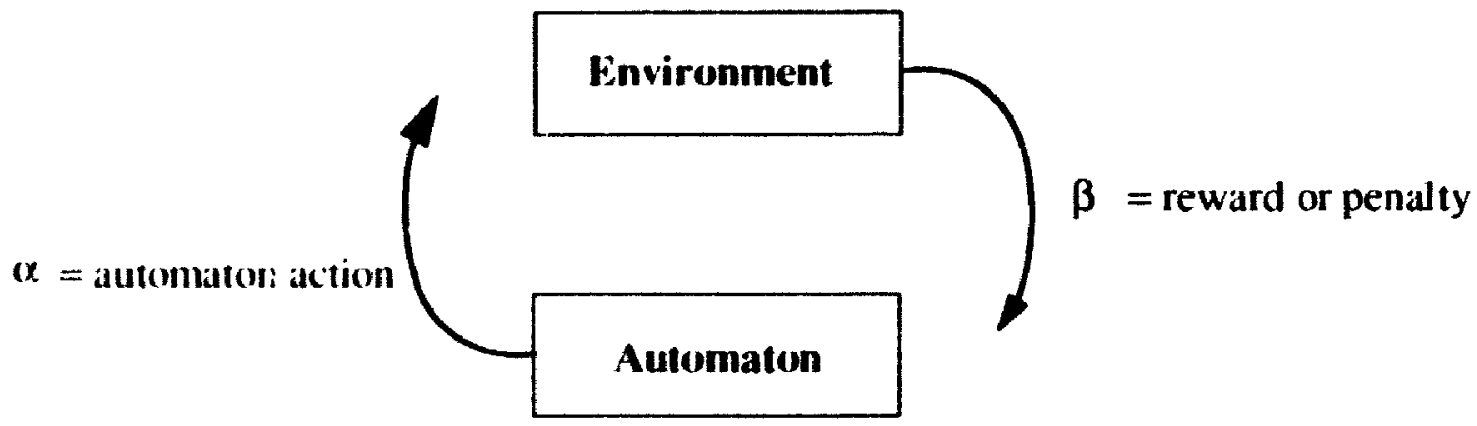

\section{Figure 29 Automaton interaction with its environment}

The automaton learning process can be described as follows. The automaton is offered a set of actions by the environment with which it interacts and must choose one of those actions. When an action is chosen, the automaton is either rewarded or penalized by the envirmment with a certain probability. A learning automaton is one which learns the (1ptimal action i.e. the action which has the minimum penalty probability, and eventually 
chooses this action more freyuently than other actions. The cycle af action-envirumuntal feedback is shown in Figure 29.

Stochastic learning automata can be classified into two main classes. These alte fixed structure stochastic automata (FSSA) and automata whose structures ender will timk: Examples of the former type are the Tsetlin ITsetlin. 1961. 19731. Krinsky and Kiylus automata. Examples of the latter type are called variable structure slochantic anlomata (VSSA) as a result of the time-varying nature of their transition and output malrices. In practice they are defined in terms of action-probability updating rules which are cither of a continuous or discrete nature. Examples of applications using discrete action-probability

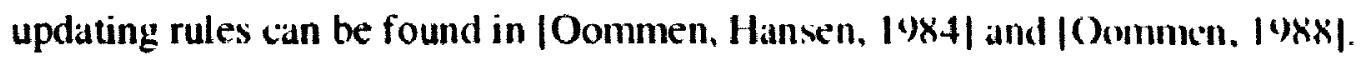

$$
\begin{gathered}
F S S A=(\alpha, \Phi, \beta, F, G) \\
\alpha=\left\{\alpha_{1}, \ldots, \alpha_{R}\right\} \\
\Phi \\
\beta=\{0,1\} \\
F \\
G
\end{gathered}
$$

is the set of actions from which automaton chooses is the set of automaton states is its set of inputs - 1 representing a penalty. 1 a reward is a matrix defining the state transition of the antomation on receiving an input. $F$ may the stochastic.

is a matrix which determines the action taken by the automaton if it is in a given slate.

The selected action serves as an input to the environment whuch gives a respome $\beta(11)$ all time $\mathrm{n}$, where $\boldsymbol{\beta}(n)$ is either 0 or 1 . This response is fed back to dhe automatom. Thus the environment penalizes the automaton with the penalty $c_{1}$, where:

$$
c_{i}=\operatorname{Pr}\left[\beta(n)=11 \alpha(n)=\left(x_{i}\right] \quad i=(1, \ldots, R)\right.
$$

Initially, the $c_{\mathrm{j}}$ are unknown and the purpose of the iutomaton is to compule them as at result of its interaction with the environment. The min( $\left.c_{1}\right)$ is the best attion, $i$. If $c_{1}$, is equal to the minimum penalty probability, and $P_{i}(n)=\operatorname{Pr}\left(x(n)=\omega_{i}\right.$ then the

1. Due to Varsharski and Vorontsova. Proof is by comblructum. 
aim is to have $P_{l}(n)=1 \quad ; P_{i}(n)=0 \quad \forall i \neq L$. i.e. the automaton learns to choose the optimal action.

A variable structure stochastic automaton learns by employing a probability updating rule such that the action probability vector at the $(n+1) s t$ time instant is computed using the probability vector at the nth time instant in conjunction with the automaton environment interaction at the $n$th time instant. Hence, if $\mathbf{P}(n)$ is the action probability vector at time $\mathbf{n}$, the VSSA is fully defined by specifying a function $\boldsymbol{H}$ such that:

$$
P(n+1)=H(P(n), \alpha(n), \beta(n))
$$

Studies have centered around two classes of function $\boldsymbol{H}$, both being linear. In the first class, $H$ is a continuous function allowing $P(n)$ to take any value between 0 and 1 . In the second class, $H$ allows $P(n)$ to take a discrete number of values in the range 0 to $I$. In the discrete case, linearity is taken to mean that the increments in probability are all equal. This latter class of automata, due to Oommen, are significantly easier to analyze ${ }^{1}$, have an expected convergence time which is finite, and appear to have superior accuracy when compared to their continuous counterparts [Oommen, Hansen, 1984].

Automata can also be broadly classified in terms of their Markovian representations. Learning automata are either ergodic or possess absorbing barriers. Automata of the former class converge with a distribution that is independent of the initial distribution of the action probabilities. This is not the case for automata that are absorbing. While absorbing automata are well suited for stationary environments, ergodic automata are ideal for nonstationary environments because they do not get locked into any particular action [Lashmivarahan. [981]. As such, this class of automata are well suited for use with genetic algorithms. as the feedback from the environment, is noisy. This point will be dixcussed in the next chapter.

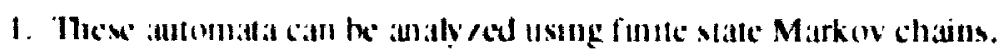


In automata theory, an automaton is said to be $\varepsilon$-optimal if the mean error (or penally) associated with it after an infinite number of interactions with the enviromunent can I $x$ mate as close to the minimum possible value by suitable choice of automatum paramelers This is defined by:

$$
M(n)=\sum_{i=1}^{k} c_{i} p_{i}
$$

where $R$ is the number of actions available to the automaton, and $c_{i}$ are the p'ilalty probabilities. So, a learning automaton is $\varepsilon$-optimal if:

$$
\lim _{n \rightarrow \infty} M(n) \rightarrow c_{m i n}+\varepsilon
$$

An automaton is said to be expedient if:

$$
\lim _{n \rightarrow \infty} M(n)<M_{n}
$$

where $M_{0}$ is the initial expected penalty.

A number of discretized linear automata have been studied. The discretized linear reward. inaction $\left(D L_{R I}\right)$ automaton is absorbing and $\varepsilon$-optimal in all random tenviromments. 'The discretized linear inaction-penalty automaton $\left(D_{I P}\right)$ is ergodic and expedient in all random environments. If artificially absorbing barriers are added to the $D I_{\text {If }}$ automatom. the autoraton is $\varepsilon$-optimal in all random environments. Finally. the discreti 2 d mulimear reward-inaction $\left(D N_{I P}\right)$ is $\varepsilon$-optimal in all random environments. These results are proven in [Oommen, 1986].

The automaton of most interest in this thesis is the two action, discreticed lincar reward penalty $\left(D_{R P}\right)$ automaton. This automaton can be shown to be ergexdic and $\varepsilon$-optinal in

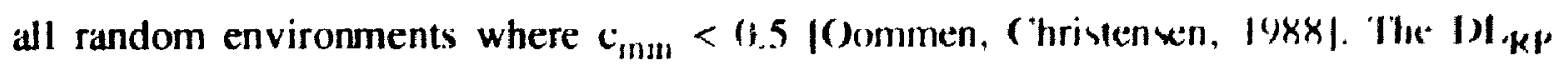


automaton has $N+1$ states where $N$ is an even integer. The automaton has a set of states $S$ $\left.=\left\langle s_{(}\right), s_{1}, \ldots, s_{N}\right\rangle$. Associated with the state $s_{i}$ is the probability $i / N$ that represents the probability of choosing action $a_{1}$. Hence the probability of choosing action $a_{2}$ is given by $(1-i / N)$. As any one of the action probabilities completely defines the vector of action probabilities, it is possible to consider just $p_{l}(n)$ with no loss of generality. The transition map for the $D L_{R P}$ automaton can be specified, given $s(n)=s_{k}$ as:

$$
\begin{array}{ll}
s(n+1)=s_{k+1} & \begin{array}{l}
\text { if } a(n)=a_{1} \text { and } b(n)=0 \\
\text { or } a(n)=a_{2} \text { and } b(n)=1
\end{array} \\
=s_{k-1} & \begin{array}{l}
\text { if } a(n)=a_{1} \text { and } b(n)=1 \\
\text { or } a(n)=a_{2} \text { and } b(n)=0
\end{array} \\
s(n+1)=s(n) & \text { if } s(n)=x_{0} \text { or } s_{N} \text { and } b(n)=0 \\
=s_{1} & \text { if } s(n)=s_{0} \text { and } b(n)=1 \\
&
\end{array}
$$

It can lo: secn from the above that an automaton in state $s_{0}$ has to choose action $a_{2}$, and similarly if it is in $s_{N}$ it must choose action $a_{l}$. The change in action probabilities can be written for ()$<p_{l}(n)<1$ as:

$$
\begin{aligned}
& \begin{array}{ll}
p_{1}(n+1)=p_{1}(n)+1 / N & \text { if } a_{1} \text { is chowen and } b(n)=0 \\
\text { or } a_{2} \text { is chosen and } b(n)=1
\end{array} \\
& \begin{array}{ll}
p_{1}(n)-1 / N & \begin{array}{l}
\text { if } a_{/} \text {is chosen and } b(n)=1 \\
\text { or } a_{2} \text { is chosen and } b(n)=0
\end{array}
\end{array}
\end{aligned}
$$




$$
\begin{aligned}
p_{1}(n+1) & =p_{1}(n) & & \text { if } f_{1}(n)=0 \text { or } 1 \text { and } l(n)=0 \\
& =1 / N & & \text { if } p_{l}(n)=0 \text { and } l(n)=1 \\
& =1-1 / N & & \text { if } p_{1}(n)=1 \text { and } l(n)=1
\end{aligned}
$$

The increments between probability states are thus $I / N$ - all incroments |xilly cyual. Furthermore, if $c_{1}<c_{2}$, the automaton has no absorbing barriers except in the degencrate cases when $c_{1}=0$ or $c_{2}=1$. With the observation of these limitations. the Markov chain is ergodic and that the limiting distribution of being in any state is independent of the corresponding initial distribution. Thus the $p /(n)$ behave as a homogenemus Markuv chaill.

The DL $L_{\text {RP }}$ automaton is the only known ergodic symmetric linear reward penalty VSSA that is $\varepsilon$-optimal is any random environment ${ }^{\prime}$ where the minimum penalty is less than 0.5 . It is considerably better than the $L_{R P}$ which is at best expedient |Lakshmivarahan, II) $X \mid$ |.

In the context of this thesis, the $p_{l}(I)$ correspond to the probability of performing a crossover as a specific bit location and $p_{2}(n)$ is the probability of nor performing a crossover at that same bit position. The environmental response is determined by the evaluation of the fitness function for a given offspring - a reward being given for a liller individual, a penalty for an inferior offspring. While intraduced here, the integration of automata and the crossover operator is the subject of the next chapter and is dealt with in depth there.

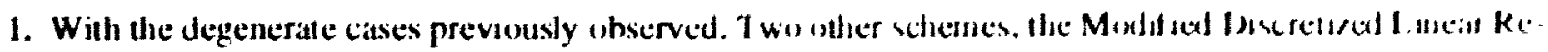

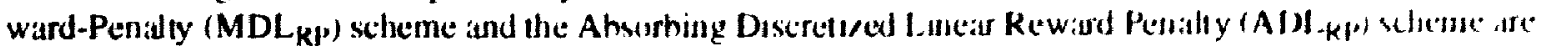

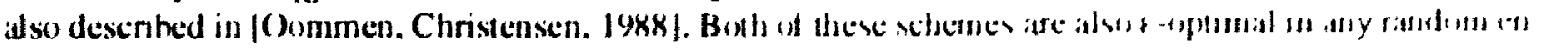
vironment - without the limitat ions on $c_{\text {min }}$ previously descrited. 


\section{Adaptive Crossover Using Automata}

As has been shown in previous chapters, the genetic algorithm and other search strategies have benefitted from modifying the search parameters themselves dynamically. This has been achieved by extending the problem representation in order to encode the search parameter and by providing feedback from the environment in order to reward successful operator action.

In this chapter we build upon the ideas drawn from previous work in adaptive operator probabilities by associating a linear reward-penalty automaton with each object variable ${ }^{\prime}$ in the representation and by providing input to the automaton based upon the fitness of the off spring produced by the action of the adaptive crossover operator.

The adaptive uniform crossover operator is an extension of the uniform crossover operator [Syswerda, [9x9]. In the uniform crossover operator, decisions as to whether bits are exchanged between parents are made on a bit-by-bit basis. The uniform crossover operator has been shown to be superior to one and two-point crosisover in a large number of environments through extensive empirical studies. The uniform crossover operator has been chosen here because of its lack of reprexentational bias i.e. defining length has no meaning for this operator and that adaption can oxcur at the bit level. Chapters 2 and 4 describe the mechanics and theory of the uniform crossover operator.

1. In this implementation. avery hit. 
This chapter consists of four sections. The first section describes the representation necessary for genetic algorithms using the adaptive unifom crossover operator, and the modifications required in the algorithm needed (1) employ it. The second section describes the adaptive multi-point crossover operator. The third section presents the results obtained by using adaptive uniform crossover operator on all functions in the function test bed. Finally. the fourth vectic!n discusses the results obtained in the experimental study.

\subsection{The adaptive uniform crossover operator}

The basic idea of the adaptive uniform crossover operator is to inlentify groups of bits within the bit string that should be kept together when crossover ikcurs. Stated another way, we wish to leam from previous crossover operations in order lo minimize schema disruption in future crossovers. This is shown schematically in ligule 30.

$F_{1}$

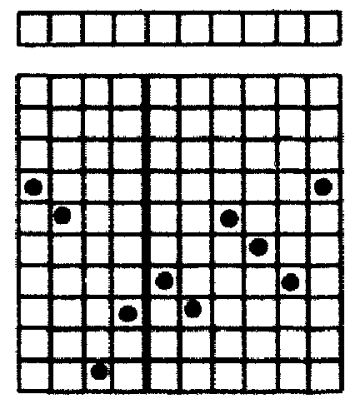

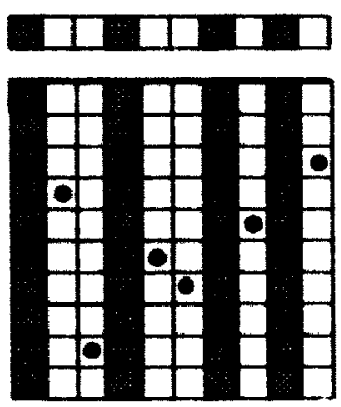

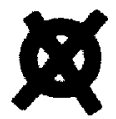

$$
F_{3}>F_{1} \cdot F_{2}
$$

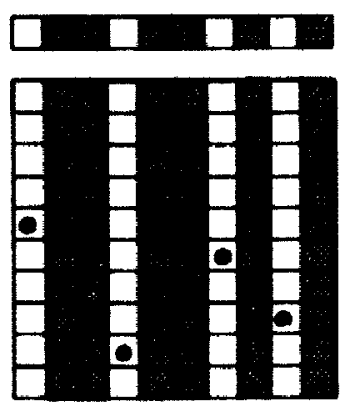

$\mathrm{F}_{4}<\mathrm{F}_{1}, \mathrm{~F}_{2}$

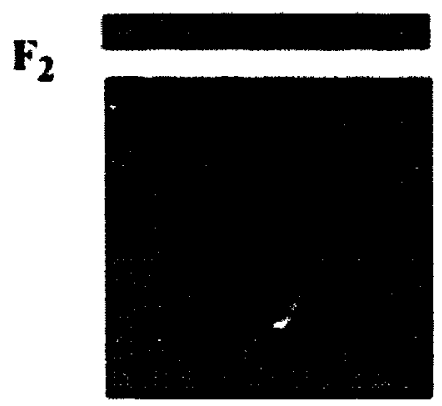

Figure 30 Schematic of adaptive uniform crossover operatur 
In Figure 30) the basic bit string representation is augmented at each bit position with an automaton, shown as a vertical set of states. The $\bullet$ associated with each automaton indicates the current state of that automaton. Each state of the automaton maps to a probability of crossover value for that bit string location. State changes occur as a result of feedback from the environment; in this case the environment is provided by the change in value of the fitness of the offspring when compared to the parents.

The principle used here is that bits which were kept ogether during crossover: and generated superior offspring, should be kept together in future crossover operations'.

Similarly. bits which were kept together during crossover, and generated inferior offsyring. should not be kept together in future crossover operations ${ }^{\prime}$.

Finully, hits which nere kem logether during crossover, and generuted offspring with no discernible fitness change, may or may not be kept together in future crossover operations'.

The al gorithm used for updating automaton state can be described as:

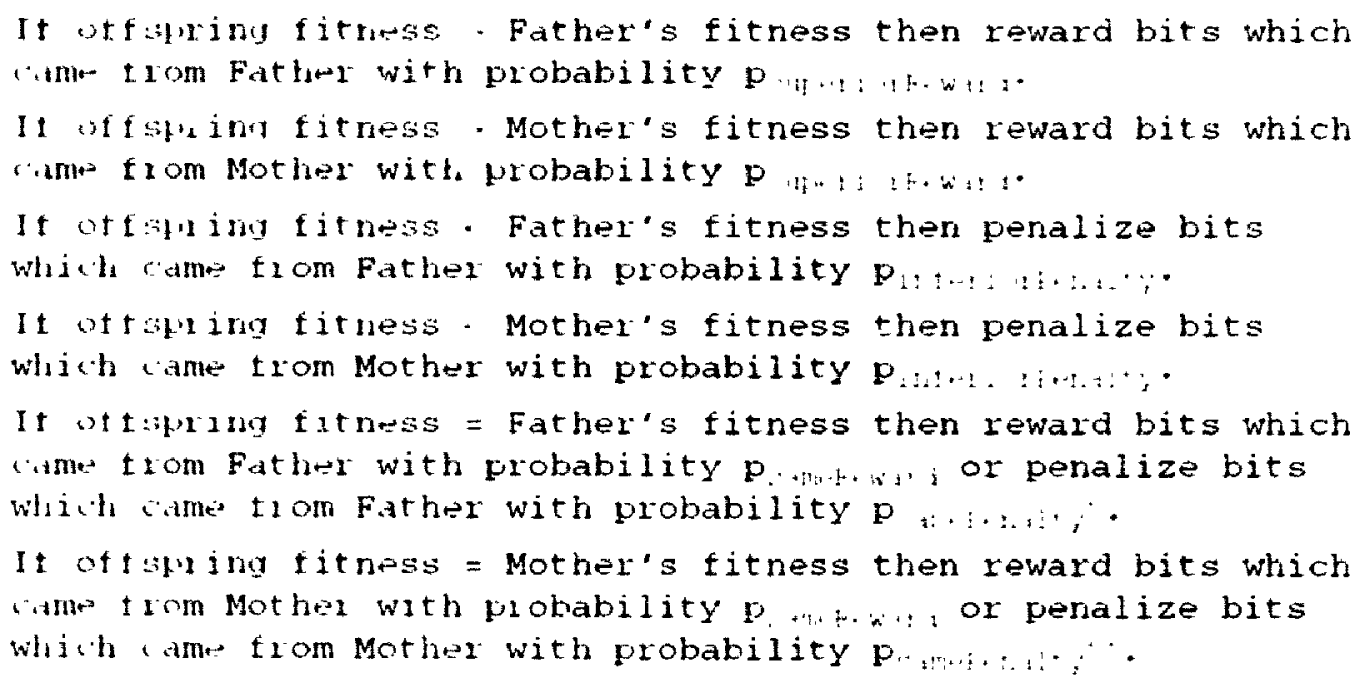

Subject to the limitation $p_{\text {sameRew }}$ ard $+p_{\text {samePenally }} \leq 1$.

1. Nattsucully specuking.

2. Do notlung with probahility 1-Psancke-wad d-Psomet'enalty. 
A reward is given to an automaton if its fitness exceeds that of a parent by the thanting

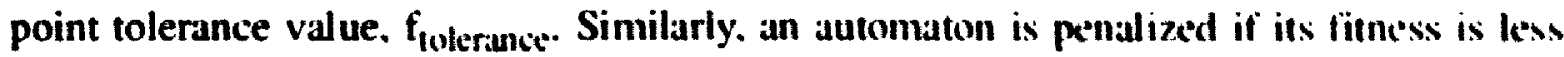

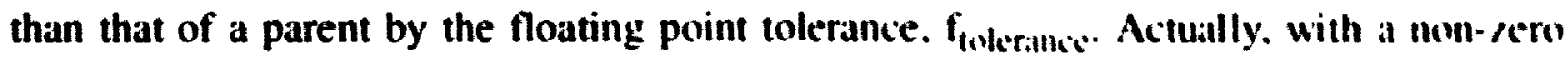
$f_{\text {tolerance }}$ value, a dead-band controller has been included in the system. A reward implies that the automaton moves from state $i$ to state $i+1$. Similarly. a penalty implies that the automaton moves from state $i$ to state $i-1$. If the automaton is in state n prior in a rewart. nothing happens. If the automaton is in state zero prior to a penal ty heing applied, nothing happens. This is implied in Figure 30 and more explicitly (kemonstrated in Figure 31 .

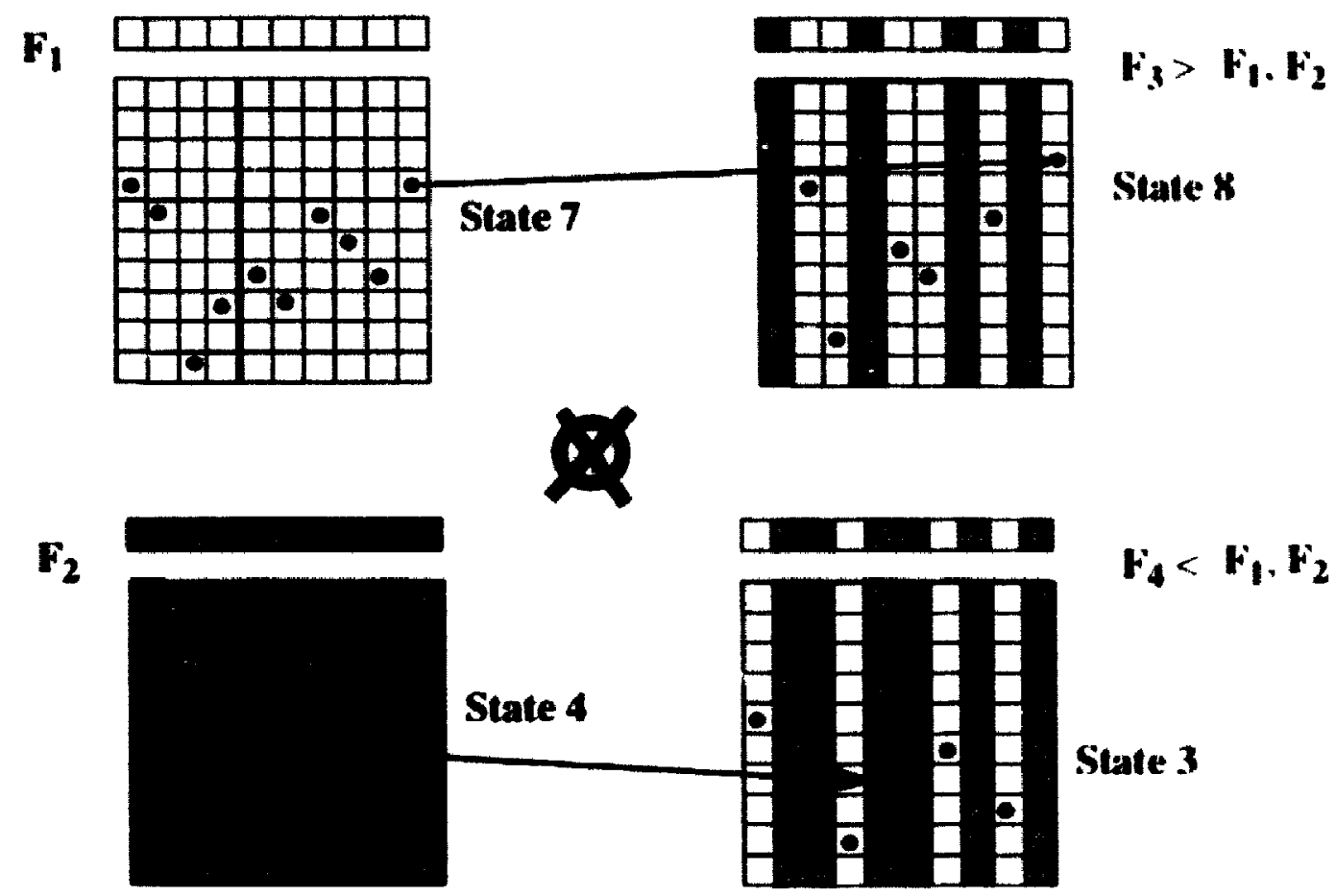

Figure 31 Automaton reward-penalty scheme

From the above algorithm it can be seen that all bits will be rewarded if the fitness of all offspring exceeds the fitness of both of its parents. If the fitness exceeds that of onte parent. but is inferior to that of the other, certain bits will be rewarded while others will be: penalized. The rewarding and penalizing of an offspring in the situation where its fitmess is equal to that of one or both parents is useful in preventing stagnation of the crosserver probability at a non-optimal value. The environment gives us incomplete informatust, in 
that in certain situations bits will be rewarded which were not the direct cause of improvement. Similarly, for the same reason. bits may be penalized in error. However. over many trials the tendency is for more correct information to be imparted by the environment than incorrect information. If this were not so, the basic genetic algorithm would not work and the Schema theorem would be in error.

The adlaptive uniform crossover operator is also modified from that used in the case of the Syswerda uniform operator. In the case of the uniform crossover operator, a random integer from the set $\{1,1\}$ is chosen. If the result is 1 , the bits are exchanged; otherwise nothing happens. With the adaptive unifom crossover operator, the probability of crossover for the two parents is first obtained using:

$$
p_{x o r r}^{i}=p^{i}\left(s_{i}\right)
$$

where $\boldsymbol{\rho}^{j}$ is the function mapping automaton state to a probability value and $s_{i}$ is the state of the automaton asscxiated with the ith bit. The function $P^{i}$, being a probability, is constrained to lic in ( $(1,1)$ and is monotonic increasing ${ }^{\prime}$. It is a discrete function, but could be represented by a noisy function if a degree of unce: tainty needs to be added to the automaton.

The crossover at a particular bit ocurs if the following condition prevails:

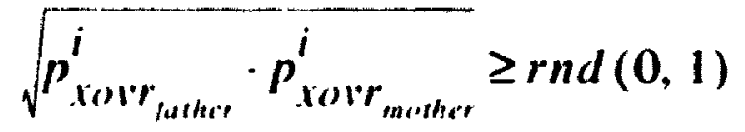

Namely. if the geonetric mean of the adaptive crossover probability values exceeds a randomly generated probability value from the uniform distribution. crosiver incurs. Otherwise. bits are copied directly to the offspring. An

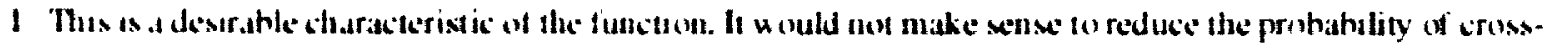

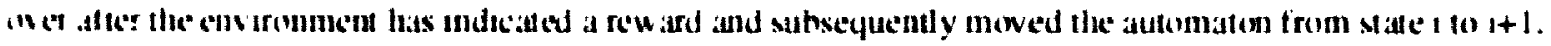


alternative formulation of the crossover operator was lo use the arithmetic moull as shown below:

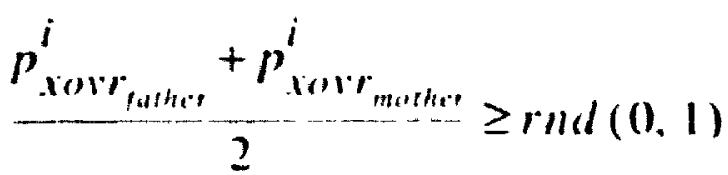

It can easily be shown that the geometric mean of two numbers is less thalli the arithmetic mean which, in the context of the above, means that the adiptus crossover operator is more conservatice - exchanging fewer hits - when using the geometric mean.

With the extended representation chosen, the action of mutation IIou lials all effect upon not only the object variable bit but also the associated atumbatin. It we take mutation of a bit to be the tlipping of that bit, then when Iogglerl. the automaton is affected because the automaton state is a function of the value of the bit at that location. If the bit is flipped. logically we loxe all information on the state of the automaton and it should therefore be resct. Being ciscutially unknown, the state is set to a random value.

The initial states of all automata were set to be the same such that the probahility of crossover equalled 1.5 - identical to the uniform operator'. One extension proposed is lo initialize the automata randomly, thereby injecting further diversity into the population as suggested by Bäck |Bäick. 1991].

\subsection{The adaptive multi-point crossover operator}

The adaptive multi-point crossover operator is similar in concept to the adaptive unitum crossover operator and the same extended representation as shown in Figure 30 and lifune 31 is used for the encoding. 
However, the meaning of the state of the automaton now changes. The state of the automalon now maps to a weight as shown in the equation below:

$$
h^{i}=H^{i}\left(s_{i}\right)
$$

where $h^{\prime}$ is the weight associated with bit 1 . alld $t^{t}$ the mapping of automaton wate to but weight. The probability of crossover at any given bit is then given by:

$$
p_{i n r}^{i}=\frac{h^{i}}{\sum_{i=1} h^{i}}
$$

For single puint crossuver. the crossover point is chosen probabilistically from the distribulion defimed by the $p^{i}$ vall values. For n-point crossover, the $n$ crossover pu.ints are chosen probabilistically from the same distribution without replacement. That is. if point ons is chosen for the first crosisover point, it is unavailable for selection for the second or subsequent crossover points.

The algorithm for rewarding or penalizing automata remains unchanged from that used for the adaptive uniform crossover operator.

\subsection{Experimental setup and results overview}

The adaptive unifom crossover operator was tested against all functions in the function lest hed $-f_{1}$ to $f_{24}$. The results were compared using equivalent genetic algorithm search parameters to the uniform crossover operator. A modified version of Cirefenstelle's exenetic algorithm experimental tool GENFSIS was used for all

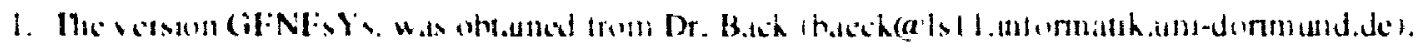


experimental runs. In all genetic algorithm runs, the same basic parameters were shosen.

These are shown in Table 12:

Table 12 Common experimental parameters

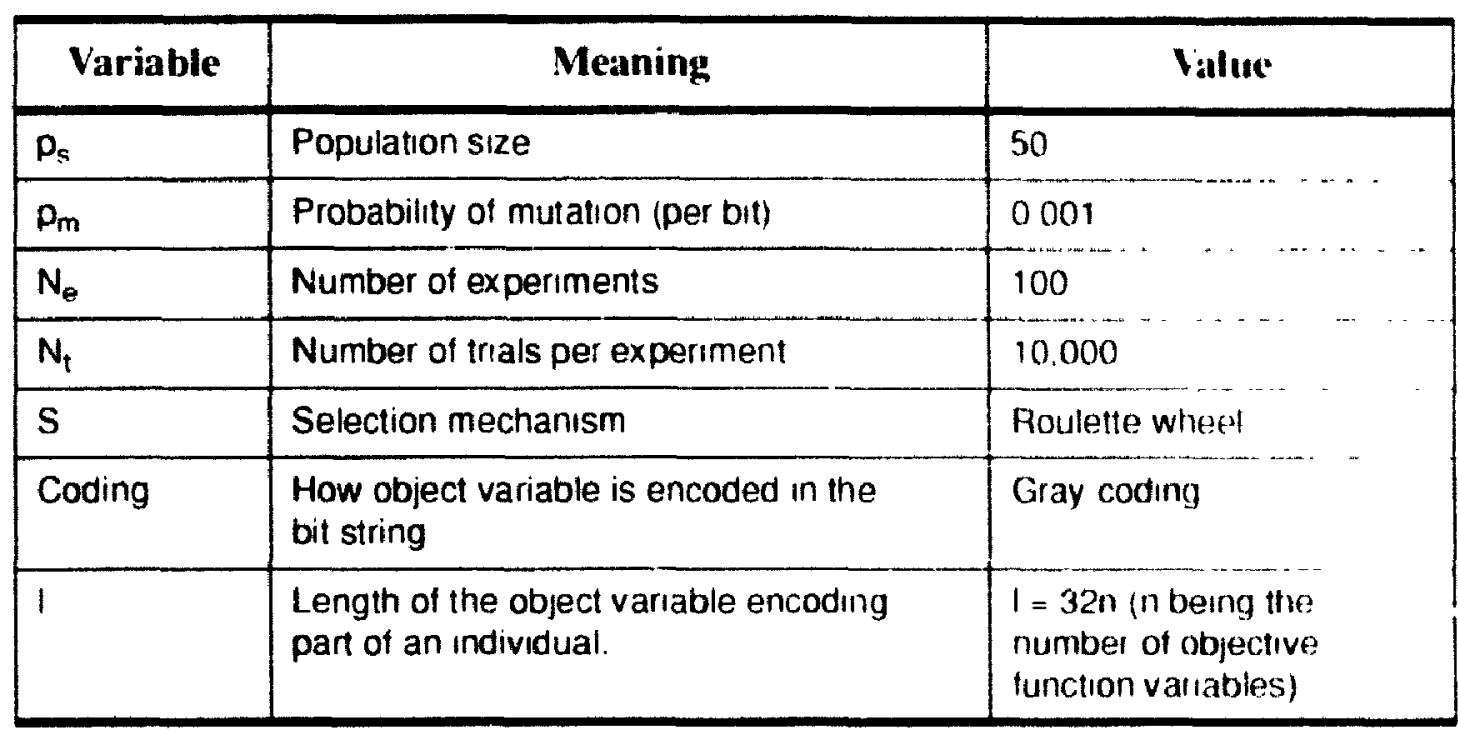

The crossover probability for the uniform crossover opetator $u$ as wel 10 11.5. Hhe geometric form of the crossover exchange operator was used lo decide whether hit wise crossover was to take place and $P^{i}$ had the functional form:

$$
P^{i}(1)=.1 / n ; 1=0, \ldots, n
$$

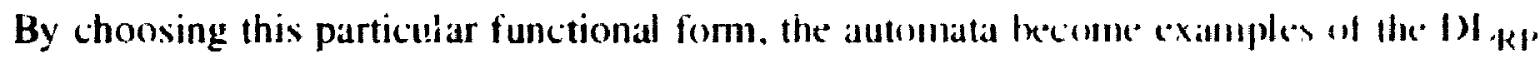
automaton; with the properties dexribed in the previous chapter. Kestallang the alwove equation in the form of probability updating rules. we see that the probablity mestases by $1 / n$ if we exchanged bits and the off spring containing the bit was more stecesstul Han the parent. Similarly, if we did not exchange bits and the off spring containimg the but was less successful than the parents, the probability of crossover incseases by $1 / n$.

If an exchange of bits took place and the oftepring contammg the bill was less successful than the parent, the probability of crossover decreases by $1 / 11$. Fimally. If fite bis ale mol 
exchanged, and the offepring containing the bit was less successful than the parents, the prohability decreases by $1 / \mathrm{n}$.

Hence the function spans the probability range $(0,1)$ and is linear. The values of the various automata probabilities are shown in Table 13:

Iable 13 Adaptive crossover operator parameters

\begin{tabular}{|c|c|c|}
\hline Variable & Meaning & Value \\
\hline$\rho_{\text {supariorRaward }}$ & $\begin{array}{l}\text { Probability with which an automaton } \\
\text { will be rewarded if the offspring is fitter } \\
\text { than the parent. }\end{array}$ & 1.0 \\
\hline Pinfuromipenatity & $\begin{array}{l}\text { Probability with which an automaton } \\
\text { will be penalized if the oftspring is less } \\
\text { tit than the parer.t. }\end{array}$ & 1.0 \\
\hline $\mathrm{p}_{\text {samePanalty }}$ & $\begin{array}{l}\text { Probability with which an automaton } \\
\text { will be penalized if the offspring is as fit } \\
\text { but no fitter than the parent. }\end{array}$ & 0.0 \\
\hline$P_{\text {samatenward }}$ & $\begin{array}{l}\text { Probability with which an automaton } \\
\text { will be rewarded if the offspring is as fit } \\
\text { but no fitter than the parent. }\end{array}$ & 0.0 \\
\hline Imlewance & $\begin{array}{l}\text { Floating point tolerance used to decide } \\
\text { whether an oftspring is more or less fit than } \\
\text { its parent(s). }\end{array}$ & 0.0 \\
\hline
\end{tabular}

In the three charts which follow in the next section, the hest-at-trial ${ }^{1}$ fitness is plotted. Other interesting statistics which were reviewed, but not included due to space constraints, were conline and offline performance, and population diversity. The $y$ axis is the difference in fitness values of the best solution in the population between the non-adaptive and adaptive runs. The $x$ axis is the trial number. Therefore, if the curve falls below the $x$ axis, the adaptive run is superior to the non-adaptive run ${ }^{2}$.

\subsection{Discussion}

In this study, naive parameter settings for the various automaton probabilities were chosen II order to test the robusiness of the adaptive approach with the purpose of the study being

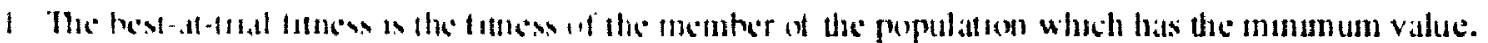

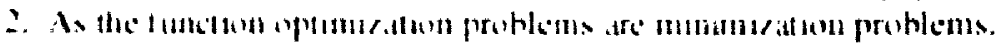


to achieve better' solutions in a given number of trials. Similarly, a simple, lincill probability mapping function was chosen. The results presented in Appemliv B ilealy demonstrate the utility of the adaptive uniform crossover operater to assist in genetic search. Setting aside $f_{10}$ for the moment. of the remaining 2.3 functions. 1.3 shows improvement. 6 show no discernible improvement and 4 are actually Morse. While the improvements seen in certain functions are small. in others. stch as $f_{2}$. $f_{11}$ and $f_{17}$. they are considerable. Certainly, the belief that more "good" than "had" infomation is provided by the environment seems to be supported by the empirical results thatt the adaptive uniform crossover operator is able to produce betler solutions than by using the uniform operator alone.

Phot of f $^{3}$ versus Trial difference between adaptive and mon-adaptwe rum

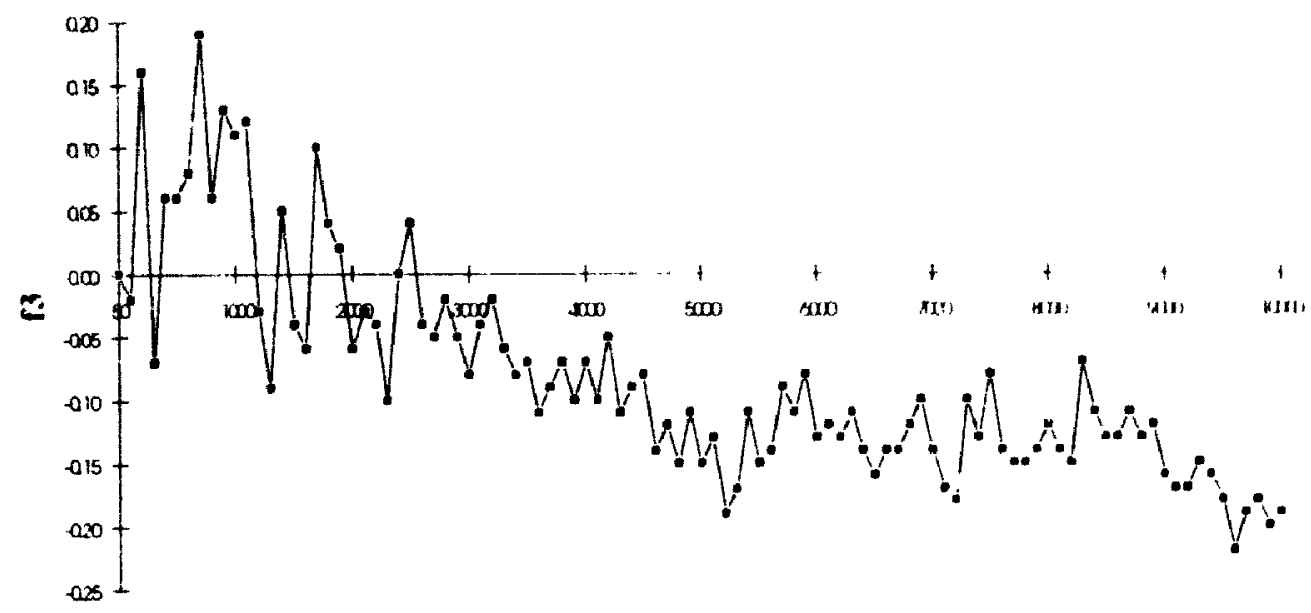

Trial

\section{Figure $32 \quad \boldsymbol{f}_{3}$ difference between non-adaptive and adaptive runs}

As can be seen from the above chart, the adaptive run is mitially worse than the min! adaptive run. This is due to the fact that the information being fed back fom the environment is actually very poor - we are exchanging tow many hits in the cursuve' operation and schema are not being correctly identified. However, as the run progresses,

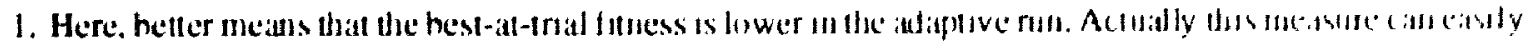

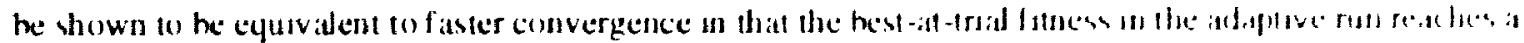

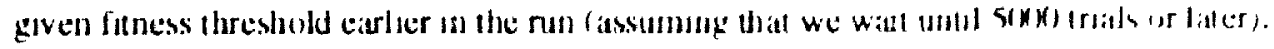


the value of information being fed back from the environment improves and the DL $L_{R}$ automata begin to converge. That is, subsets of hits which should be exchanged during irosisoler begin to amerge. The value of the feedback from the environment evidently increases rapidly as the adaptive run is superior to the non-adaptive run beyond $25(0)$ trials.

Ihot of fo versus Trial difference between adaptive and mon-adaptive rums

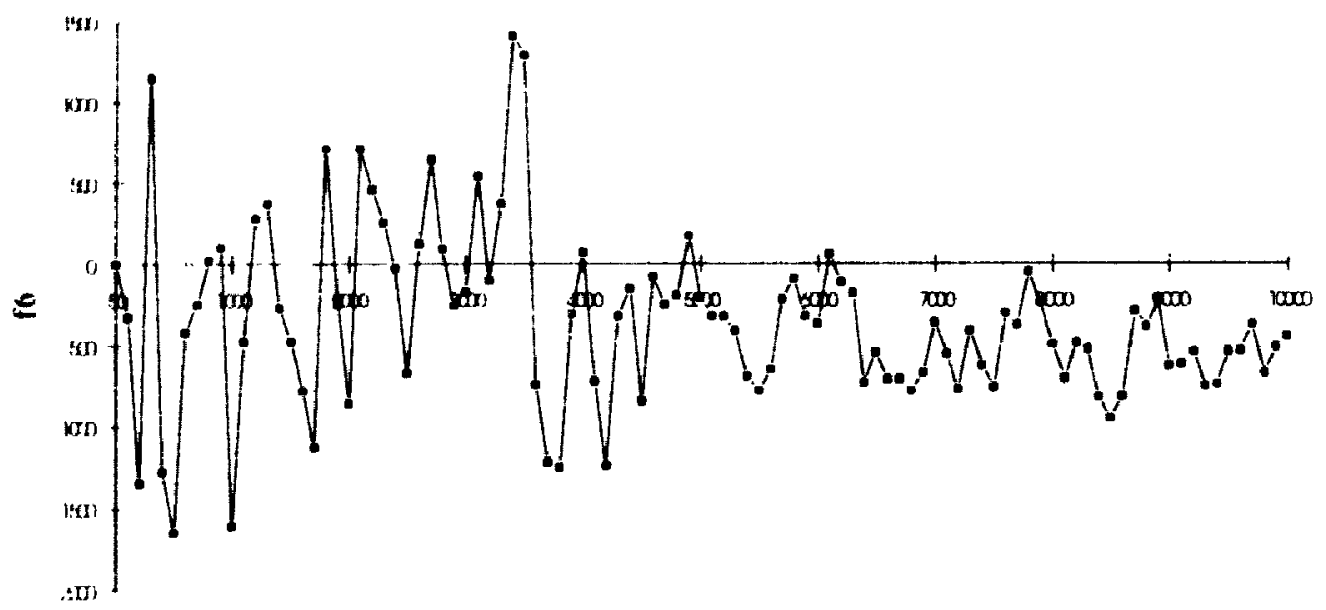

Trial

\section{Figure $\mathbf{3 3} \quad f_{6}$ di'ference between non-adaptive and adaptive runs}

The ahore chart again clearly shows the same pattern as seen in Figure 32. Initially, the value o: feedback from the environment is quite small leading to sizeable differences Ixtwe'n the non-adaptive and adaptive runs. Sometimes the adaptive run is better, and at other moments the non-adaptive run is superior. However, as we converge. the value of fectback from of wironment increases considerably and the automaton at each bit position begins to afy the correct bit patterns to keep together during the crossover operation. In the above chant. the adaptive run is clearly superior to the non-adaptive run after approximately $6(x)$ trials. Stated another way, although the feedback from the environment has initially been quite poor. the automata have recovered in order to increase the joint access probabilities for bits responsible for improved fitness. 
What is happening here is that we are initially tou optimistic in our rewadropendly scheme. We should probably have a relatively low value for the Puprouken.und ams Pinferiorpenalty probabilities initially and have them increase as the population comverges.

A contrary indicator to the previous statement can be $x^{2}+n$ in furction $f_{10}$. Iunction $f_{10}$ is interesting because it exhibits behaviour which, during the early part of the run. strongly supports the view that the automata are identifying schema which are part of lenter and

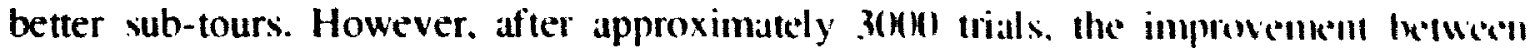
adaptive and non-adaptive runs starts to degrade until at ofton trials the IW. rums hase identical best-at-trial fitness values. Thereafter. the non-adapivive rum sxhibits superin

Plot of flo versus Trial for adaptive run

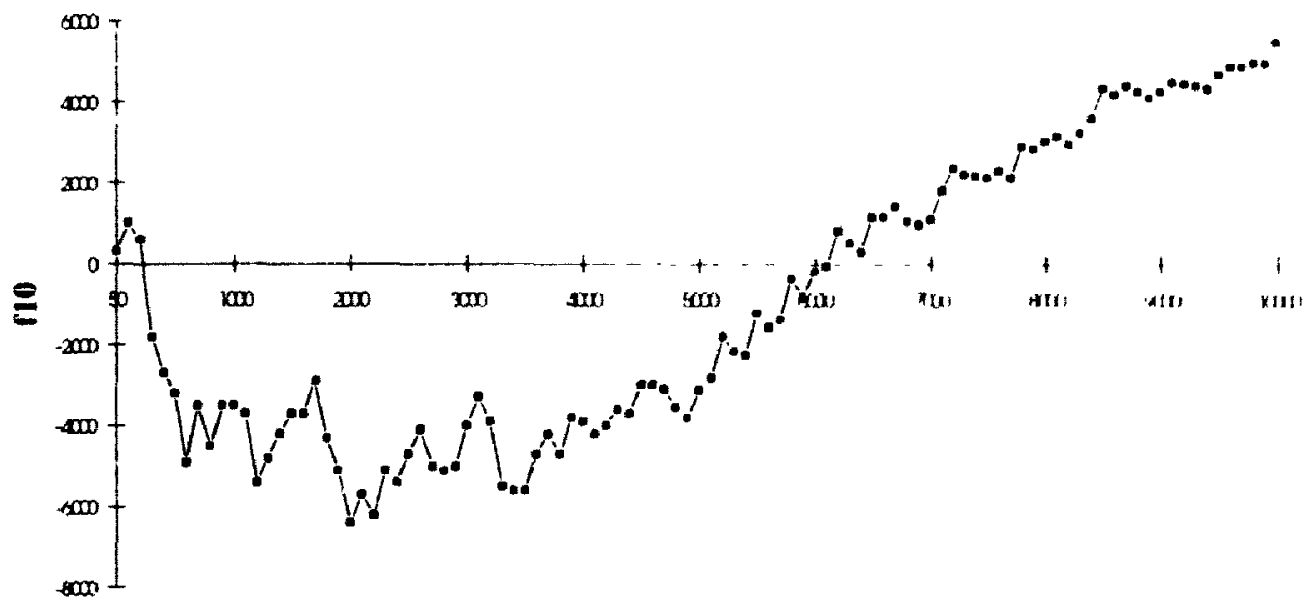

Triul

\section{Figure $34 f_{10}$ difference between non-adaptive and adaptive runs}

performance. This is due to a combination of two effects. Firstly, the Travelling Sallewnan Problem encoded in $\mathrm{f}_{10}$ is a permutation or cycle problem.

Censider, for a moment, a 5 city tour. Then the tour 1-2-?.4-5-1 has an identic al length to the tour 2-3-4-5-1-2. However. the bit string representations of these Iwo louls is quille

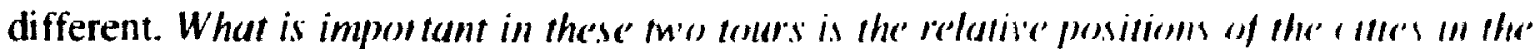

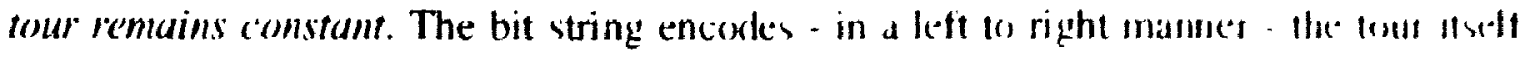


However, the automata associated with the bits in the bit string encoding the tour are designed such that the absolute values of the bits are important. The constant shuffling of bits to make a valid tour ensures that the feedback from the environment will not be correct and therefore the automata state changes based upon erroneous information.

Secondly, a problem of stagmation can occur. While this is not restricted to the Travelling Salesman Problem, it is probable that this caused the steady decay in relative fitness when compared to the non-adaptive case. The stagnation problem occurs when the automata are in states which map to very high or very low probability values. If, in these situations, litness improve ents are not seen in the offspring, no changes to the automata will be ohserved and the tendency will be for the same bits to be exchanged in subsequent trials.

Ohviously, the model described earlier in the chapter can deal with this problem by setting the $P_{\text {simmekewatrl and }} \mathrm{p}_{\text {siuncPenalty }}$ probabilities to be non-zero. Similarly. setting Psupwrnorkeward or pinleriorpenalty to be values other than one would ensure that the stagnation problem could be monitored by not al ways rewarding superior or penalizing inferior offspring. By making the various automata transition probabilities nondeterministic we are making the statement that the environment is providing noisy information, which we should treat accordingly.

Using the naive probability settings dexcribed earlier, we can see that the feedback from the environment can deceive the automata early in a genetic al gorithm run. This tends to indicate :iat gradually increasing the reward/penalty probabilities might improve algorithm performance. It should also be noted that in the random initial population. approximately one half of the bits are exchanged during crossover. This is an extremely large number of bits, containing potentially many schema. We would expect that the automata would perform better when fewer bits are exchanged. This implies that initializing the automata such that significantly fewer bits are exchanged early in the run might improve performance as the schema being identified would be smaller. 


\section{Conclusions}

Genetic algorithms have been proven to be a powerful search prexcelure capallse of solving problems in a diverse range of application areas. Although powerful. the setting of parameters to control the search procedure - the sia of the population. the values of probabilities controlling crossover and mutation remains a time consuming process with little but heuristics 11 aid in thetr chrices. No comprehensive theory exists in order to predict opturnal sellings for wall control parameters.

Control of population diversity, or convergence control. has also becoll ideutificel in it problem in genetic algorithms where the selection procedure causes excessive loss of genetic material early in the genetic experiment. Research has identified litmess scalmg as a means by which allele loss can be controlled, but this makes the choice of firmess function more difficult than it already is. Automated control of population diversity 1 considered to be highly desirabl:. This thesis has proposed a mutation operator whose probability is a function of the diversity of the population and provided empirical evide-nce to support the claims for the utility of this operator in a genetic al gurithm covirommont.

In a classical genetic algorithm, learning occurs at one level. Namely, oblect variables change under the influence of the mutation and crossover operallos and are guided by selection. Neither the operators. nor the probabilitics with which they are applied, change as the algorithm searches for an optimal solution. Ilowever, it has been shown that other search mechanisms, such as evolutiomary stalegies, " $x \cdot n \cdot 4$.jt considerably by being able to adapt control parameters as the search p.. neededs. 
This thesis has reinforced the desirability of having the genetic algorithm evolve optimal parameters for search. At the very least, the genetic algorithm should be able to start with "poor" parameter values and improve upon them as further trials lake place.

The focus of adaption has been the identification of highly fit schema and the minimization of schema disruption when applying the crossover operator. While research has shown that the uniform crossover operator does not disrupt "long" schema as much as does multi-point crossover, the operator is unable to pick out fit schema as they emerge during the search process. As such, the uniform crossover operator is unable to assist in the "growth" of scherna which the Schema Theorem tells us is the basis of success for all genetic algorithms.

This thesis proposes extensions to the uniform crossover operator in order to hive the crossover probibility adapt as genetic search proceeds: identifying bit patterns which should be kept together i.e. schema identification. The basic principle is to increase the prohability with which the bits remain together when mated with another string in some future trial. An adaptive crossover operator has been proposed. requiring extensions Io the basic representation for any problem wherein an automaton encodes the probability that a given bit will be exchanged with the other parent under the action of the modified unifom crossover operator.

A comprehensive set of experiments has been performed in order to verify that improvements to the basic genetic algorithm can be made. From these experiments it has been concluded that the basic adaptive crossover operator works well for problems where the bits have absolute value - such as is the case in function optimization - but in problems such as the Travelling Salesman Problem - where the relative bit positions are important - the automata are mislead by the enviromment. causing inferior performance to be observed.

lintegrating automata with genetic algorithms has shown promise, as this thesis has demonstrated. However. like all research, with new knowledge comes new 
questions. As such, the new adaptive unifom crossoser operater has introduced mell dimensions of complexity into an already complex sy'stem.

Further work is obviously warranted in order to answer the yuestions which this fle'sis has raised.

\subsection{Direction Of Future Work}

The scope for further work is considerable. Future worh cill he clansitical in Ihe following way:

- automata investigations

- parameter investigations

- theoretical study

- operator investigations

- integration with other adaptive operators

- application to genetic programming

These points are briefly discussed in the next six sections.

\subsubsection{Automata Investigations}

In this thesis, only a single type of automatori has been used in experiments and II has shown promise in assisting in adaptive parameter specification. (Other automata. stull as reward only, or penalty only, should be investigated in order to determine the it effectiveness in improving genetic search.

Also, this thesis has considered automata whose prohability updating rules are of a discrete nature. However, the probability updating rules might be molified in such a way as to make them continuous - perhaps depending upon the degree to which the child is

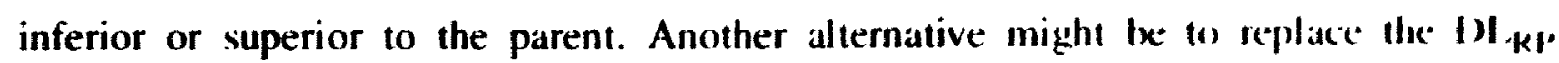
automaton with the $\mathrm{MDL}_{\mathrm{RP}}{ }^{\prime}$ automaton as the latter exhibits superior propertices in "noiser" environments.

1. See [Oommen. Chrstensen. 1968| page 454. 


\subsubsection{Parameter Investigations}

During the course of experimental investigation of the adaptive uniform crossover operator, a single set of parameters were selected. This was done in order to assess the robustness of the algorithm - given that the selection of parameter values was particularly naive and did not use the full power of the automaton!. This sometimes led to stagnation of the adaption and degraded the efficiency of the crossover operator. By enabling the component of the operator which rewards or penalizes the operator in the situation where offspring have the same fitness as their parents, it is strongly believed that such stagnation woul I be reduced.

Similarly, the simple linear function chosen to represent the adaptive uniform crossover probability as a function of state is probably too simple. That is, the rate of change of probability ${ }^{2}$ is the same across all states. A non-linear function such as are used in annealing algorithms should be investigated.

Finally, the utility of the noise function - where the state probability has a degree of uncertainty associated with it - should be investigated.

\subsubsection{Theoretical Study}

This thesis has undertaken to propose and evaluate empirically - through experimentation - the value of integrating automata with a genetic operator in order to provide adaptive operators. A theoretical investigation of the properties and dynamics of this class of operator needs to be performed.

\subsubsection{Operator investigations}

Although the uniform crossover operator has been selected for consideration in this thesis. the idea of augmenting the representation with automata at each bit position and subsequently using the automata in order to determine the probibility of crossover can be applied elsewhere. For example, the one or two

I. Row ardmg or penalie Ing un the case of no parent to chuld inprovement was nol modelled.

2. The ralc of change for the latear model is just $1 / 11$. where $n$ is the number of automaton states. 
point crossover operators could be controlled by these autumatis as descritud in section 8.2. Obviously. experiments need to be perfomed in order to verify the utility of this and other such adaptive operators.

Also. an adaptive operator which is able to deal with the Travelling Salcesmall Iroblem should be designed.

\subsubsection{Integration with other Adaplive Operators}

This thesis has concentrated on enhancing the crossover operator alone. Provious studies [Bäck,1991] have indicated that an adaptive mutation "pkeralor cau significantly improve genetic search. Sturlies should te perfommed in orrler w determine whether the integration of these two clasises of adaptive (ipkratur provide still further improvements in genetic search.

\subsubsection{Application to Genetic Programming}

Genetic programming is a recent invention and has shown comsiderable promise in solving problems where an ill-defined or unbounded representation is required. However, as with most genetic algorithms, control parameters are statk and are chosen on an ad hoc basis.

Associating an automaton with each node in the tree would allow lor Iwo possibilities. Firstly, the probability of crossover at any stle Ixecomes adaplive and that promising sub-trees, or sub-programs can emerge. Secondly, alutumatic function definition is possible in that when a node accumulates a given fiaction of the probability mass, it means that the sub-program should become a function in its own right and the probability mass redistributed amongst all nodes in the tree.

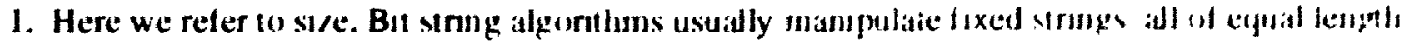




\section{References}

|Ackley, 1987| Ackley. D.H., Stochastic Iterated Gemenic Hillclimbing. Ph.D. Thesis. Department of Computer Science. Carnegie Mellon University, Pittsburgh, PA, (1987).

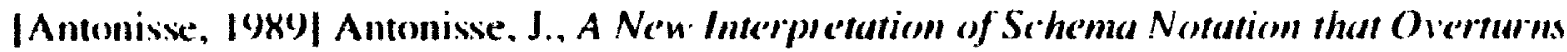
Ihe Binary Encoding Construint, in Proceedings of the Third International Conference on (ienetic Algorithms. Morgan Kaufman Publishers, Inc., Los Altos. C.4. 1989, pgs 86-91.

[Ash. [98x] Ash. T., Dymamic Node Creation in Bachpropagation Neturorks. ICS Report $x(x) 1$. The Institute for Cognitive Science. University of California. Sa: Ditgu, 198x.

|Axclrod. 1985| Axelrod. R., The simulation of genertics and chelution. A conference on Evolutionary Theory in Biology and Economics, University of Bielefeld, Germany, $19 \times 5$.

|Axelrod, 1987| Axelrod, R., The evolution of strategies in the iterated prisomes: dilemmus. In Davis (ed.) Genetic Algorithms and Simulated Annealing (pgs 3241). Pitman. London.

|Biick. |(⿻)|| Bäck. T.. Self Adaptation in Genetic Algorithms. Proceedings of the First liuropean Conference on Artificial Life. December 11-13. 1991. MIT Press, pgs 26.3271 .

|Bäck. Hoffmeister. |991| Bäck. T.. Hoffmeister. F. Ext'nded Selection Mechanisms in Grmeric Algorithms. in Proceedings of the Fourth International Conference on Genetic Algonihn s. Morgan Kaufman Publishers. Inc.. Los Altos. CA. 1991. pgs 92-99. 


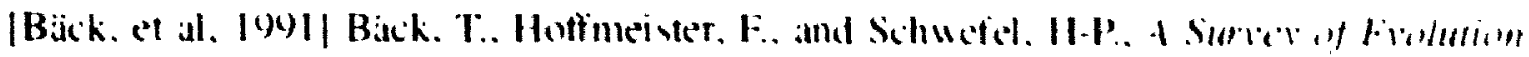

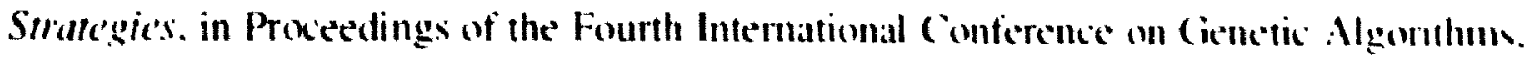

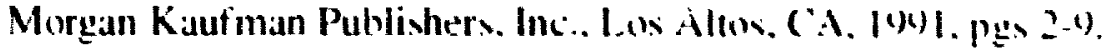

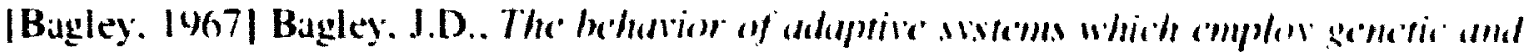
comrelation algorithms. Doxtoral discertation. Inicersity of Michigan. I)iscontation

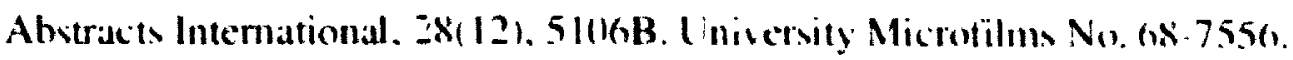

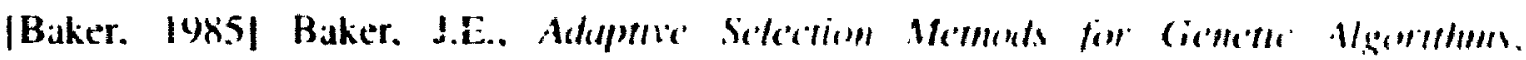
Procedings of the First International Conference on Cienelic Alporithm, I.ankme Erlbaum Publishers. Hiilisdale. NJ. 19x.5. pg? $1111-111$.

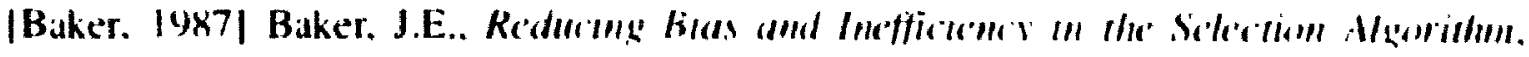
Proceedings of the Second International Conference on (iemetic Algconthmu, I.antence Erfbaum Publishers. Hillstale. NJ. 1087. pgs 14-21.

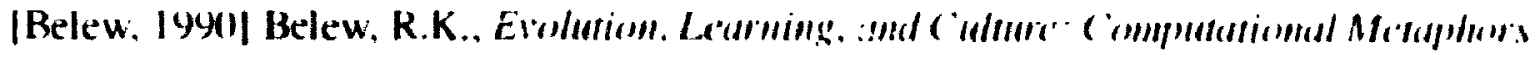
for Adaptice Algorithms. Complex Systems 4, pes II-49, fock.

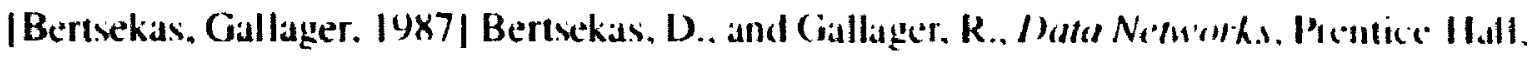
Inc., Englewood Cliffs. NJ.. $19 \times 7$.

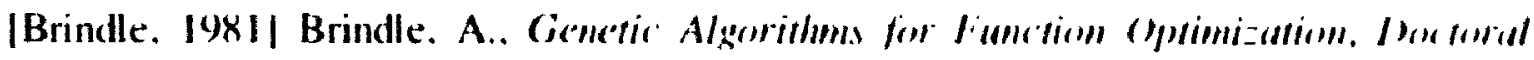
Disivertation. Iniversity of Alberta, Edmonton. 1981.

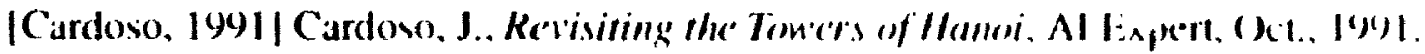

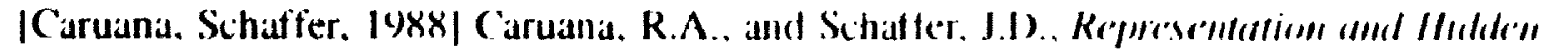

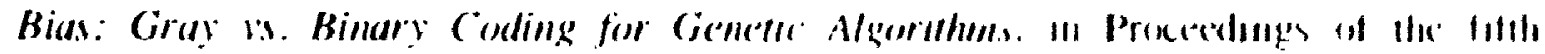

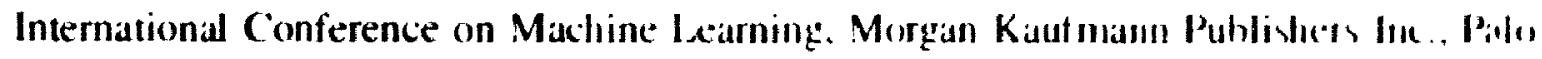
Alto. CA. pgs. 153-161.

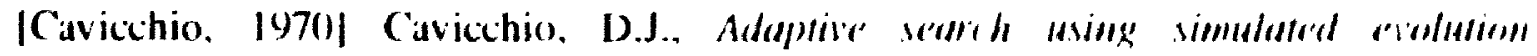
Unpublished doctoral dissertation. Iniversity of Michigan, Ann Aibor. 1970. 
|Chou and Frank, 1972| Chou, W. and Frank. H. Routins strategies for Computer Noln'ork Inesgn. Symposium on Computer-Communications Networks and Teletraffic, Polylechnic Instituke of Brooklyn. Bronklyn. NY. April 4-6. 1972.

|collius et al, I9x8| collins. N.E. Egelese, R.W., and Golden, B.L. Simulated ammedtmg all ammotuted biblongraphly, Am. J. Math. Management Sci.. 8(3 \& 4!, $2(19) \cdot 3(17.1198 \times)$.

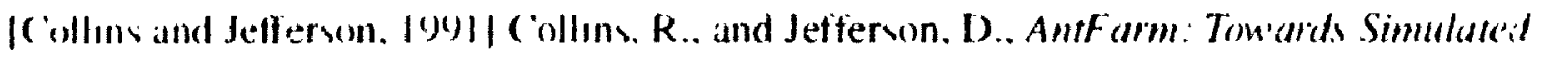
Eimlurim, in Astificial Life [I, Addison-Wesley, pgs 579-6(11, 1941.

|Coumbs, Davis, 1987| Coombs. S., and Davis. L.. Genetic Algomithme and (immmanication Link Speed Design: Theometical Coinsiderations, Proceedings of Second International conference on Genetic Algorithms, pgs. 252-256. 1987.

|Cramer. 198.5| Cramer. N.L... A Represemention for the Adaptive Generation of Simple Se'fuchtial Programs, in Procecedings of an International Conference on Genetic Algorithms and Their Applications. pgs. 18.3-188. Hillsdale. N.J., 1985. Lawrence lirlbam Associates.

|Darwin. 1859| Darwin. C.. The Origin of Specie's. London: Murray, 1859.

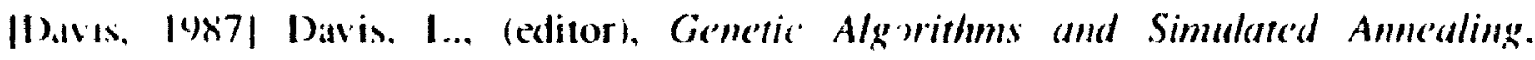
Morgan Kaufmani Publinhers, Inc.. Los Altos. CA. 1987.

|1)avis. J989) Davis. L., Adapting Operator Probahilitics in Gonetic Algorthms. in Proceedings of the Third International Conference on Genetic Algorithms. Morgan Kanfuann Publivhers, Inc., Los Altos. CA. pgs.61-69, 1989.

IDavis. 1901! Davis, L... (editor). Hamdhook of Genetic Algorithms. Van Nostrand Kcinhold. Nen York, 1991.

|lians. Coombs, 1987| Davis. L. and Coombs. S.. Genetic Algorithms and

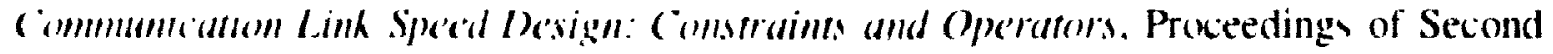
Intermathonal conference on Genetic Algorithms. pgs. 257-260, 1987. 


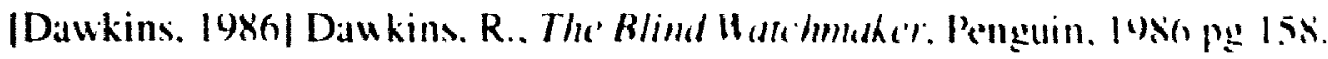

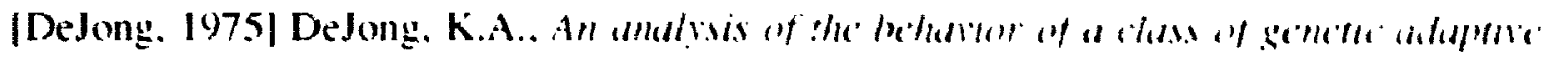

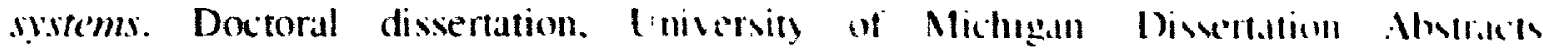

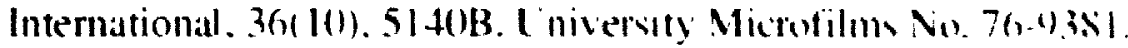

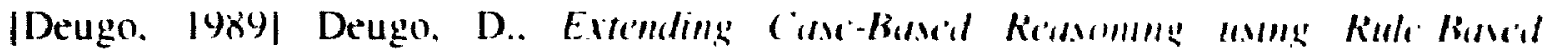

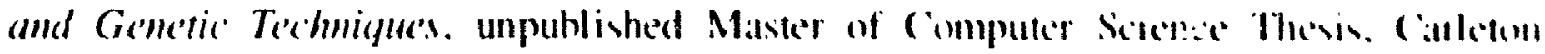
University Computer Science Department. Non. 1489.

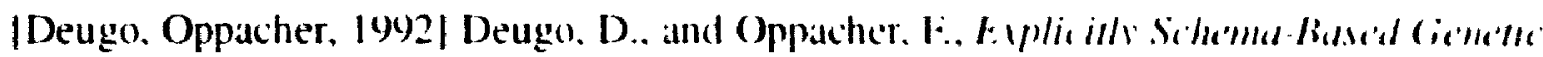
Algorithms. Procedings of the Ninth Canadian Conference an Artiticial Intelligemes. Morgan Kaufman Publishers Inc., Palo Alto, CA. pgs.4(1-5.3.. Maly, I(u) 2.

|Eshelman. et al., 1989| Eshelman, L.J.. ('aruana, R.A., and Schatfer, J.I)., Bira's in th' Fimess Lamdscape', in Proceedings of the Third Intermational C'onference on (isemetic

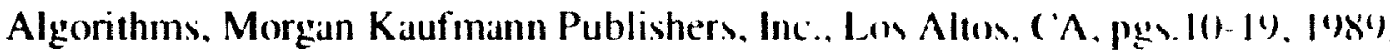

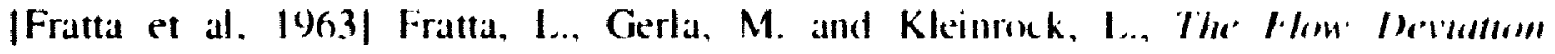

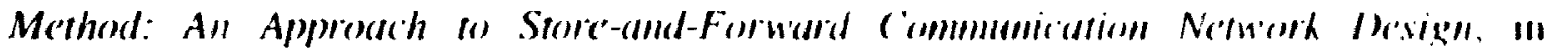
Networks, Vol. 3, pgs. $97-133$.

|Friedberg. 1958| Friedberg. R. M., A learming mulhim": Parl I. IlsM Jommal al Research and Development, 2(1), 2-13.

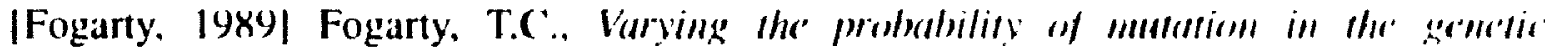
algorithm, in Proceedings of the Third International conference an Ciemetk

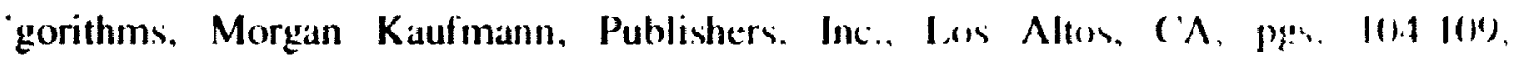
1989.

|Fogel et al, 1966) Fogel, L.J., Owens, A.J., and Wallh, M.J., Arllfrall Intelligence through Simulated Evelution, John Wiley, New York, IMn, 
|f-orrest. 1985| Forrest, S., Documentatmon for PRISONERS DILEMMA and NoKMS programs that aw the genctic algorithm, unpublished manuscript. liniversity of Michigan, Ann Arbor, 1985.

|Hujiki and Deckinson, 1987| Fujiki, C., and Dickinion. J., Using thi Genetic Aleorithm to Cienerate Lisp) Sontre Code to Solve the Prisoner's Dilemma. In Cienetic Algorithms and their Applications: Proceedings of the Second International ('onference on (ienetic Algorithms, pgs. 236-24(), Hillsdale N.J.. 1987 1.awrence Eirlbaum Associates.

|cielfand, 1987| Gelfand S.B., Analysis of simulated ammealing type algorithms, Thesis, MIT, (ambridge, MA. (1987)

|Giman and Geman, 1984| Geman, S., and Geman, D., Stochastic relaxation, Giblss distriburion and the Bavesian restoration in images. IEEE Taan.. Patt. Anal. Mate. Int. 6(6). 721.741 (1984).

|Gillies, 1985| Gillies, A. M., Machine leaming pronedures for generating image domain foumure defertors, Unpublished doctoral dissertation, University of Michigan. Ann Arbor.

|Ciold. |978| ciold, E.A. Complexity of automaton dantification given data. Information and (ontrol (37). pgs 3(12-320, 1978 .

|Gioldherg, |989) Goldberg. D.E., Genetic Algorithms in Search, Optimization and Marhinc L'aming. Addison-Wesley. Reading. MA. 1989.

IGioldberg. 19y)al Coldberg. D.E., A note on Boltzmann tournament selection for g'metic algorithms amd population-oricened simulated annealing, Complex Systems, 4. $p \operatorname{sis}+45-46(1)$

| Givldherg. ct al.. 1901| Goldherg. D.E.. Deb. K.. and Korb. B.. Dont Womy. Be Messy. in Procecedings of the Fourth International Conference on Genetic Algorithms. Morgan Kiat mann, Publishers, Inc.. Los Altos. (A. pgs. 24-30. 1991. 
|Grefenstette. |986| Grefenstette. J.J. Optimization of Cimmol Parumitar fin Genctic Algorithms. IEEE Transations on Systems, Man and cylermetics, sale 16.1 (January-February. 196), pgs. 122-128.

|Groot, Wuertz, 1990| Groot. Claas de., and Wurth, D., Optimuing cimpler

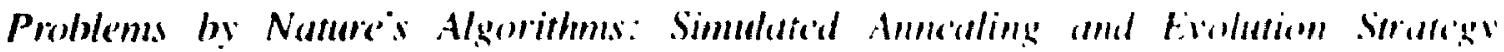
a Comparative Study. Proceedings of the tirst Workshop an Parallel Protilem Solving in Nature, Springer Verlag, New York. pgs 445-454. $19 \%(1$.

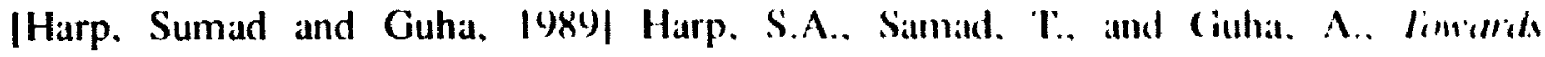
the Genetic Symthesis of Netural Nerwerks, in Proceedingss of the Mhird International Conference on Genetic Algorithms, Morgan Kiafmann. Publivilus. Inc., Los Altos, CA, pgs. 3601-37\%, 198\%.

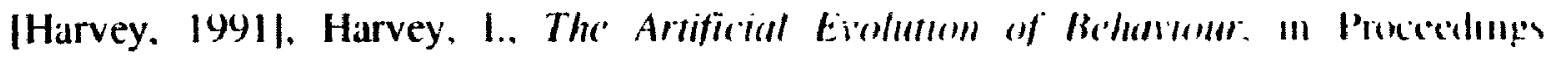

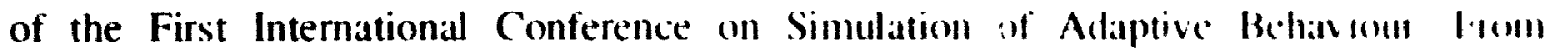
Animals to Animats, pgs 4(0)-4(1), MIT Press, Mass.

|Hesser, Manner, 1991 | Hesser, J., and Manner, K., Towards an optimal mutation probability in genetic algorithms, in Parallel Problem Solving trom Natture. Vui. 496 of Lecture Notes in Computer Science, Springer Verlag. Bcrlin. (k.tolk.r. 1990, Berlin, pgs. 23-32.

|Hoffmeister, Bäck, 1990)| Hoffmeister, F., and Bäck. T., Gometic Algorillmms and Evolutionary Strategies: Similarities and Differemes, Proceedings of the Ist Work shop om

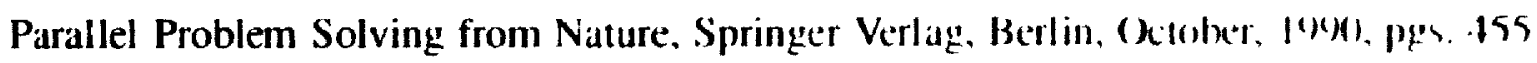
469.

|Holland, 1975] Holland, J., Adaptanion in Nedtutal amd Artificial Siverms, University of Michigan Press, 1975.

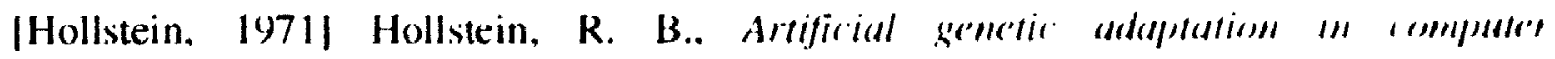
control systems. Doctoral dissertation. Unversity of Michigan. Darsertatum Abstracts International 32(3). 1510B. University Microtilms No. 71 23.773. 
|lngher, Iy以) Ingber, i.., Statistical mechanical aids to calculating torm structure models, Phys. Rev. A 42(12), 7(157-7(164 (1990)).

|lngher et al, I99Ial Ingber, L.., Wehner, M.F., Jabbour, G.M.. and Barnhill. T.M.. Application of stanistical mechanies methodology to term-structure bond-pricing morkls, Mathl. Comput. Moxklling 15(11). 77-98 (1991).

|lngher. 1091b| Ingber, L.. Siatistical mechanics of neocortical interactions: $A$ walims paradigm applied as chectrocmephalography, Phys. Rev. A 44(6), 4017$4(06)(199)$ ).

lingher. 10021 Ingber. L.. Genesic mesoscopic neural netu'orks based on statistical merhamics of meocortical interactions, Phys. Rev. A 45(4), R2183R2186(10)2).

Ilngber, Rosen, 19921 Ingber. L.. and Rosen, B.. Genetic Algorithms and lery fias Simulaned Annosaling: A Comparison, Mathematical and Computer Modelling, 16(11) pgs $87-10(1,1902$.

|lngber. 10931 Ingber, L.. Simmlated ammeding: Practice rersus theory. Statistics and (omputing, to appear. 1993.

|Janikow. Michalewicz, |q9|| Janikow, C.Z., and Michalewicz. Z.. An Experimemtal: C'mmbarisom of Rinary and Flodting Point Represcentations in Genetic Algorithms. in Proceedings of the Fourth International Conference on Genetic Algorithms, Morgan Kaufimann, Publishers, Inc.. Los Altos. CA. pgs. 31-36, 1991.

|Jefierson, et all. lo9l| Jefferson. D.. Collins, R., Cooper, C., Dyer, M.. Flowers, M. Kort. R., Taylor, C.. and Wong. A.. Evolution as a Theme in Arrificial Life:

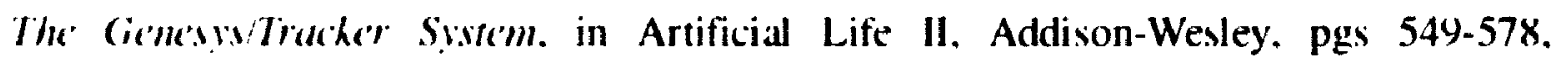
|(s) |

fJohurme et al. 1987| Johnson. D.S., Aragon, C.R., M.Geoch, L.A., and Schevon. (.. Optimization by simulated ammealing: an experimental evaluation (IJ)ts / and 2). Report. AT \& T Bell Laboratories, Murray Hill. N.J. (1987). 


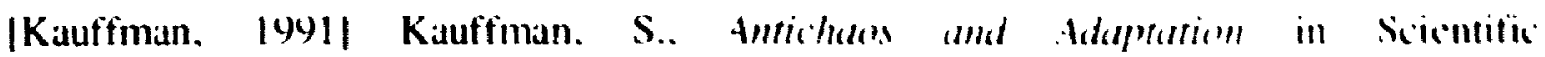
American. August. 1991, pgs 78-8;.

|Kirkpatrick et al. 198.31 Kirkpatrick, S., Ciclatt. (.1).. and Viccill. M1.1.,

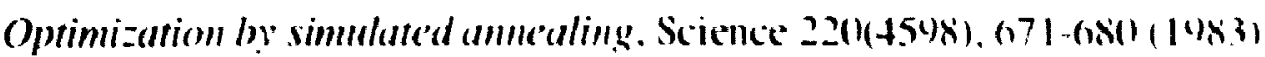

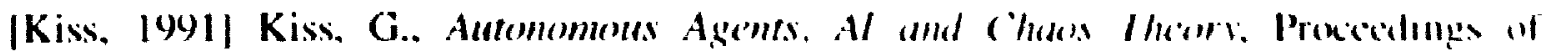
the First International Conference on Simulation of Adiptisc Mehavious. Mall Mass. Mass, 1991 pgs 518-524.

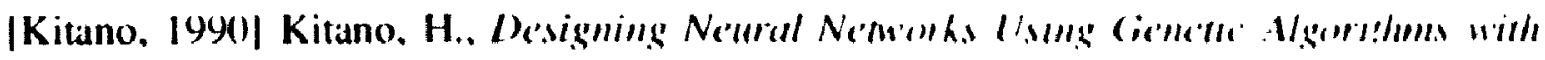
Graph Generation System, in Complex Systems 4, ngs 461 476, 1146).

|Kleinrock, 1975| Kleinrock, L.. Qucueing Systems. Iil.l. New Yink: Juhu Wiley anul Sons.

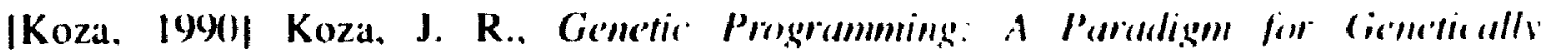
Brecoding Populations of Computer Programs a Sollo Problems. Stantunal University Computer Science Department lechnical reporı STAN C S $4 / 1114$.

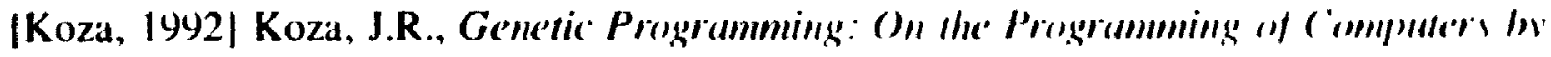
means of Natural Selection, MIT Press, Mass, 1992.

[Lakshmivarahan, 1981] Lakshmivarahan. S.. Learning Algorilhms Therol and Applications, Springer-Verlag, New York, $\mid 981$.

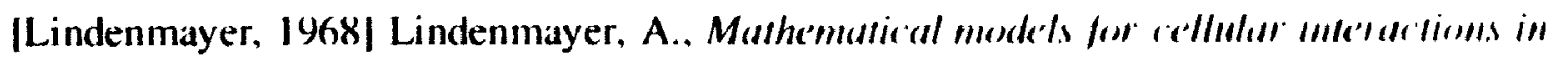
developme'nt, J. Theor. Biol., 18(1968) pgs. $2 \times(1-20 \%$.

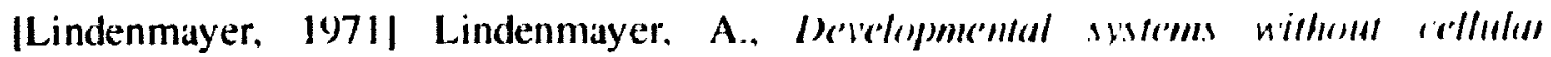
imeractions, their languages and grammars, J. Theor. Biol., 301(1971) pys. 455.48.4.

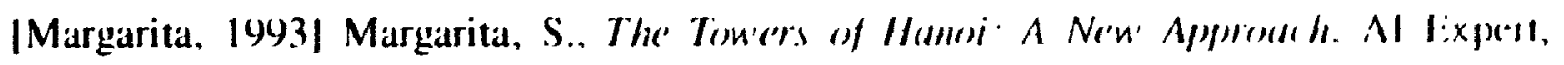
March, 1993, pgs 22-27. 
|Michalewicz, |942| Michalewicz, Z., Gonotic Algorithms + Data Structures = Evolution Progerams. Springer Verlag Publishers, 1992.

|Michalski, 1983| Michalskı, R.S., A theory and methodolosy of inductive learming, Machinc Learning. Morgan Kaufmann Publishers Inc., Los Altos. CA. $19 \times 3$.

|Michalski et al., 1986| Michalski, R.S., Carbonell, J. G., and Mitchell. T. M.. Rscaping Rrittleness: The Possibilities of General Purpose Learning Algorithms Applied " Parallel Rule-Based Systems, in Machine Learning II. Morgan Kaufmann, Publishers, Inc., Los Altos, CA. 1986.

|Miller and Todd, 1989| Miller, G.F.. and Todd. P. M., Designing Neural Networks using Ih' Cienctic Algorithm, in Proceedings of the International Joint Conference on Artificial Intelligence, Volume 1.pgs. 762-767. 1989.

|Mitchell. 1982| Mitchell. T.M., Generalization as search, Artificial Intelligence 18, $14 \times 2$.

|Montana and Davis, $1989 \mid$ Montana, D., and Davis, L., Training Fe'd Fom'ard Neural Nofw'orks using Cientetic Algorithms, in Proceedings of the International Joint Conference on Artificial Intelligence. Volume 1, pgs 762-767.

|Narendra and Thathachar, 1974| Narendra. K.S... and Thathachar. M.A.L., Learning antomata - a sur's'y, IEEE Trans. on Systems, Man and Cybernetics SMC-4, pgs 323-334. 1974.

INarendra and Thathachar. 1980| Narendra, K.S.., and Thathachar, M.A.L.. On the b'harionr of a l'arming antomaton in a changing cmviromment with routing applications. IE:EI: Trams. on Systems. Man and Cybernetics SMC-10. pgs 262-269, 1980.

|Narendrat et al. 1977| Narendra. K.S.., Wright. E.. and Mason. L.G.. Application of lemming atumatha to telephome tratfic moting. IEEE Trans. on Systems. Man and Cykrnetics SMC-7. pgs 785-792. 1977. 


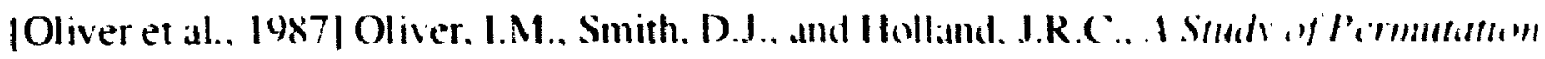

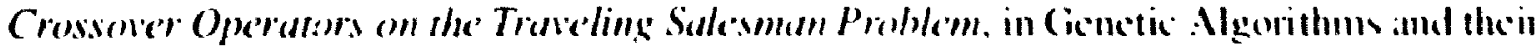
Applications: Proceedings of the Second International Conterence va Cienctle Nymullums,

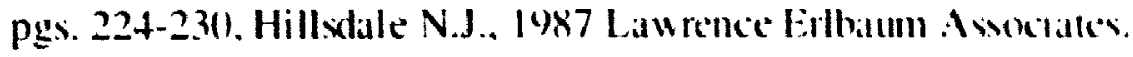

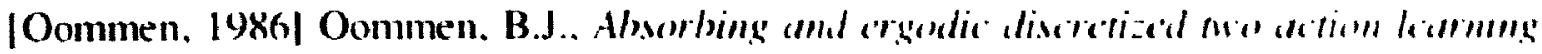

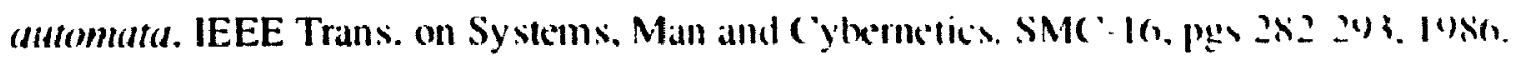

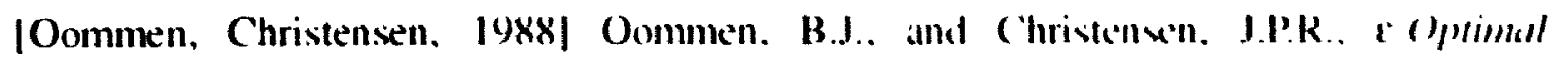

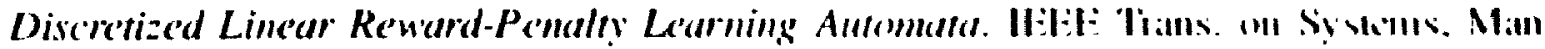
and Cybernetics. SMC-18. ps 4.51-458, 148x.

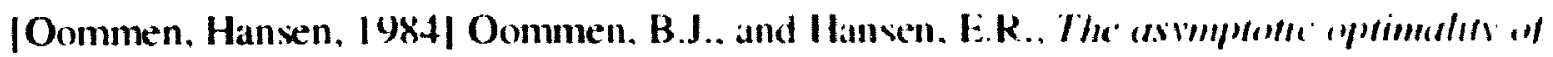

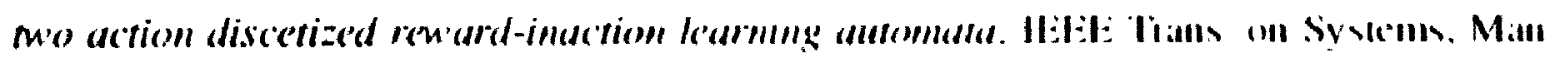
and Cybernetics. SMC-14. pg. 542-545, 1984.

|Pan, Wang, 1991] Pan, H., and Wang. I.Y., The Bamdwidh Allociation of A7M Ihrollgh Genetic Algorithm, Globecom Proceedings, pgs. 125-129, |\%)1.

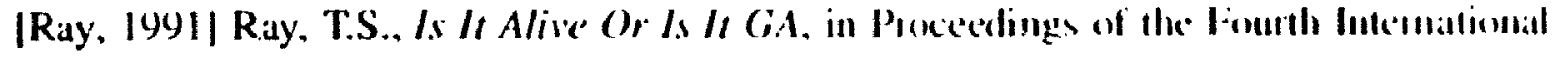
Conference on Genetic Algorithms, Morgan Kaufmann Publivhers. lnc. Pgs. 527 5.4. 1991.

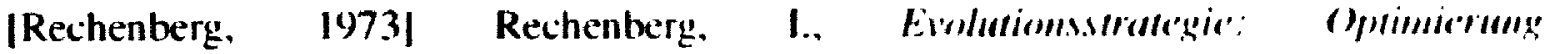

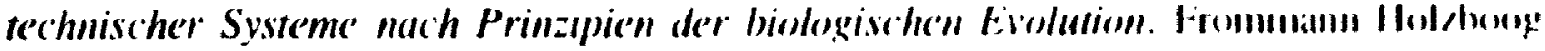
Verlag, Stuttgart, 1973.

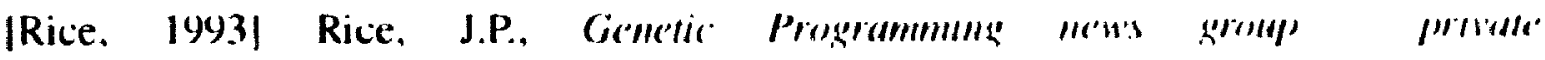
commanication, March, 1943.

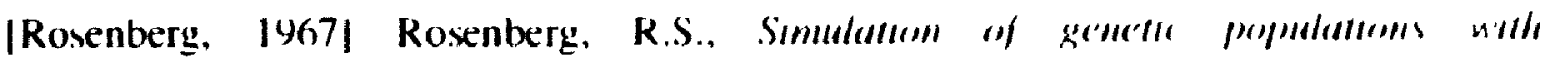
biochemical propertics, Doctoral dissertation, liniverstty of Miclugran Dissertation Abstracts International. 28(7). 2732H. linversity Microfilms Vis. $67-17.836$. 
|Kumelhart et al. |986| Rumelhart. D.E.. McClelland, J.L., and the PDP Kescarch Ciroup, Parallel Distributed Processing: Explorations in the Microstruchures of Cognition, Volume I: Foumdations, MIT Press. Cambridge MA.. IOX6.

[Samuel, [959] Samuel, A.L.. Some studies in machine learning using the game of rheck('r. IBM Journal of Research and Development, 3(3), 210.229.

|Sanmuel, 1967| Samuel, A.L.. Serme studies in machinc kearning using the yame of cherkers, Rocemt progress IBM Journal of Research and Development. $11(111,6011-617$.

|Schaffer, 1987| Sch:ffer, J.D., An Adaptive Crossover Distribution Mechanism for Cirmeric Algorithms, Proceedings of the Second International Conference on (ienetic Algorithms. Lawrence Erlbaum, Hillsdale, NJ. pgs. 36-40, 1987.

|Schaffer et al, 1989| Schaffer, J.D., Caruana, R.A., Eshelman, L.J.. and Das. R.. Combrol paramerers Afferting Online Performance of Genetic Algorithms, in Proceredings of the Third International Conference on Genetic Algorithms. Morgan Kaufmann Publishers. Inc. pgs. 51-61), 1989

ISchwefel. 19751 Schwefel. H-P., Evolutionsstrategie und mumerisithe Optimicrung. Disiertation. Technische Universitat Berlin, Berlin, May 1975.

ISchwetel. 1977| Schwefel, H-P., Numerische Optimicerung von ComputerMokllk'n mincls der Evolutionsstrategie. Interdisciplinary systems research; 26. Bimauser. Basel. FRG. 1977.

|Sichwetel. 1977| Schwefel. H-P.. Namerical Optimization of Compater Models. Wiley, (hichester. 1981.

|Simpson, |(9)(X)| Simpson. P.K.. Artificial Neural Systems, Foundations, Paradigms, Applications and Implame'ntations. Pergamon Press. 1990. 


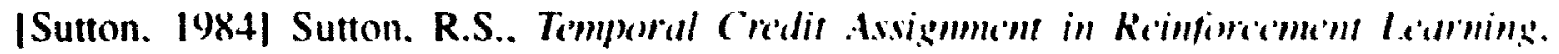

Doctoral Dissertation, COINS Technical Report KA-112. Iniversity of Massachusetts. Amherst. 1984.

|Syswerda. 1989| Syswerda, G.. Unifom crossune\% in genenic algarithms. In Proceedings of the Third International cimference on ciellelle Algorithms. Morgan Kaufmann Publishers. Inc. pgs. 2-4. 1984

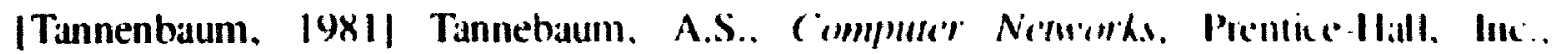
Englewood Cliffs, NJ.. 1981.

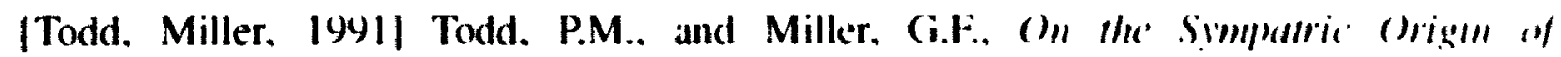
Species: Morcurial Mating in the Quicksiluer Madel. In Proceolings of the Fourth International Conference on Genetic Algorithms, Morgan Kanumam Publishers, Inc. pgs. 547-554. 1991.

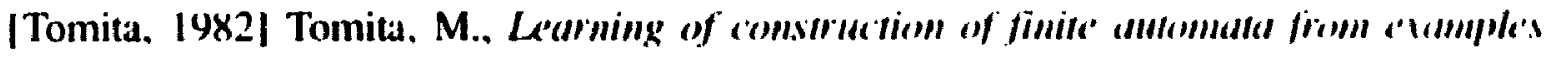
using hill-climbing. MS thesis. Carnegie-Mellon University. 1982.

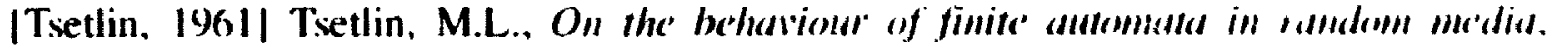
Automat. Telemek. 22. pgs 1345-1354, 1961 .

|Tsetlin. 1973| Tsetlin. M.L.. Automatum Theory and Modelling of Biologrical Sustoms. Academic Press. New York and London, 197.3.

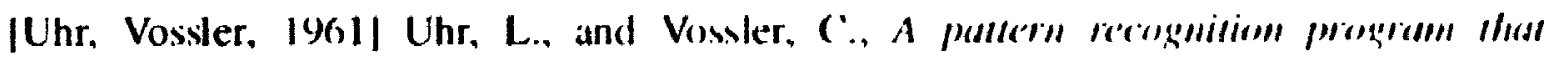
generates, enaluates and adjusts its on'n opmeromers. Anmals of Now Youk Academy of Science, 5(1) 189), 555-569.

Ivan Laarhoven and Arts, 1987| van Laarhoven, P.J.M., and Aarts. |:.II.I... Simulated Annealing: Theory and Applicatioms, D. Keidel, Bondrecht, Hollatud (1987). 
|Weinberg, 197011 Weinberg. R.. Computer simulation of a living cell. Doctoral dissertation. University of Michigan. Dissertations Abstracts International. 31(9). 5312B. Iniversity Microfilms No. 71-4766. 1971.

|White, 1992| White. A.R.P., GASTE: The Genetic Algorithm Smallualk Test Environme'nt, unpuhlished manuscript.

|Whilley, IUXyal Whitley, D., The GENITOR Algorithm and Selecrion Pressure: Why Kamk-Kascd Allosation of Trials is Best. In Proceedings of the Third International Conference on (ienetic Algorithms. Morgan Kaufmann Publishers. Inc. pgs. 116-121, $198 \%$.

¡Whitley et al, 1991/ Whitley, D., Dominic, S., and Das, R., Genetic Reinforcememt Iderming with Multilaver Neural Netu'orks, In Proceedings of the Fourth International conference on (jenetic Algorithms. Morgan Kaufmann Publishers, Inc. pgs. 562-569, 1091.

|Whitley et al, I9XYb| Whitley. D.. Starkweather. T., and Fuquay. D'Ann., Sicheduling Programs and Traveling Salesmen: The Genetic Edge Recombination Operator. In Procerdings of the Third International Conference on Genetic Algorithms, Morgan Kaufmann Publishers, Inc. pgs. 133-140, 1989.

|Whilley and Hanson, 1989| Whitley. D., and Hanson. T., Optimizing Neural Nets Using Fuster: Morc Aciurate Ginetic Search, In Proceedings of the Third International Cintierence on Cienetic Algorithms, Morgan Kaufmann Publishers. Inc. pgs. 391-396, 198\%.

|Whitley and Kauth. 1988| Whitley, D.. and Kauth, J., GENITOR: A Different G('netic Algurithm, in Proceedings of the 1988 Rocky Mountain Conference on Artificial Intelligence. pgs. 118-1301. 198X.

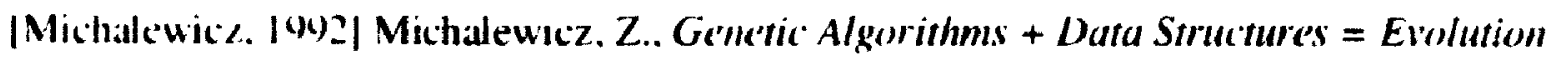
Programs. Springer-Verlag. New York, 1992. 
|Wilson, 1487| Wilson. S.W., Classifier systems amd the Animul problem. Machine Learning $2(3)$ pgs. $199-228$.

|Zhou, Grefenstette, 1986] Zhou. H.. and Girefienstetle. J.J., Indurtion of finifs

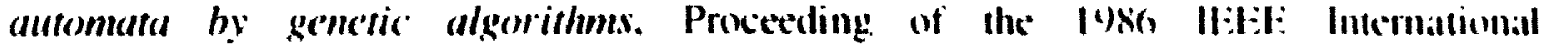

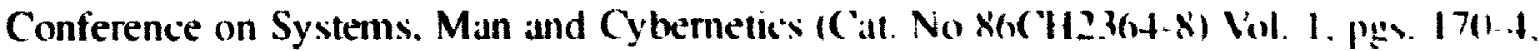
Oct. 1986 


\section{Appendix A Test Functions}

This appendix contain the set of test functions used for the experiments performed is this thesis. The functions have been designed in order to display the following properties:

1. continuous/discontinuous

2. convexinonconvex

3. unmomolal/multimodial

4. Guadralic/monquadratic

5. Iou dimensional ity/high dimensionality

6. deterministic/stochastic (or noisy)

The'x properties were first set out in DeJong's thesis |DeJong. 1975]. 
A.1 Sphere Model

$$
\begin{aligned}
& 1.11 \quad \sum ! \\
& 5.12=1,-5.12
\end{aligned}
$$$$
m i n(1,) \quad t, 1(1, \ldots .11) \quad(1
$$

\section{A.2 Generalized Rosenbrock's Function}

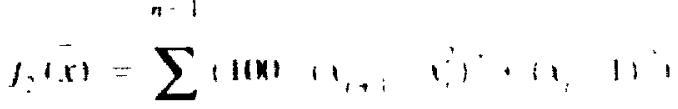

$$
\begin{aligned}
& 5.12-1+512 \\
& m m 1 /, i, i 1, \ldots, 1) \quad \|
\end{aligned}
$$

\section{A.3 Step Function}

$$
\begin{aligned}
& f(x)=11+\sum+1 \\
& -5.12<.1,5.12 \\
& m(n) f_{2} ;=1.115 .12,51 \ldots .15 .12 .51, \text { n }
\end{aligned}
$$

\section{A.4 Quartic Function with Noise}

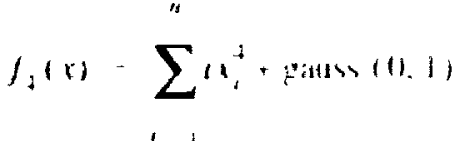

$$
\begin{aligned}
& 1.2 x-1,-12 x
\end{aligned}
$$

$$
\left.m(n) f_{i}\right) \quad f_{i}(1) \ldots(1) \quad \text { (1) }
$$




\section{A.5 Shekel's Foxholes}

$$
\begin{aligned}
& \operatorname{li}_{1,11 ;}^{1} \quad \kappa^{\prime} \cdot \sum \\
& \text { - } \sum(1, \cdots)
\end{aligned}
$$

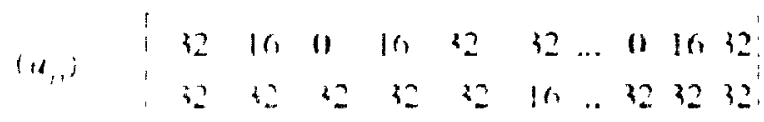

$$
\begin{aligned}
& 1,5.530,0.15 .540 \\
& \therefore ! a, n, 1, \cdots, k \text { S(n) } \\
& \text { mtnit, }=(1,-32,-32)-1
\end{aligned}
$$

\section{A.6 Schwefel's Function 1.2}

$$
\begin{aligned}
& l_{t}(1)=\sum_{1-1}^{n}\left(\sum x\right)^{2} \\
& 65,53601=65,564 \\
& m(n), 1, \quad t, 10 . . .01)=0
\end{aligned}
$$

\section{A.7 Generalized Rastrigin's Function}

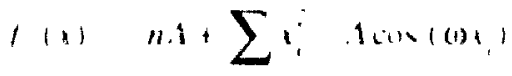

$$
\begin{aligned}
& 1 \quad 11:(1) \cdot 2 \pi \\
& 5.12 \div 1,55.12 \\
& m a n t /-1 \quad \mid(1) . . .(1)-1)
\end{aligned}
$$




\section{A.8 Sphere Model, Changing Environment}

$$
\begin{aligned}
& \sum_{1} \text { imodacin } \\
& 1,11014 \\
& \sum \cdots, \\
& \text { d } 2506 \text { (1) } \\
& \text { Imudarnen } \\
& m(n) / s) \quad\left\{\begin{array}{l}
f(0, \ldots, 0) \\
f_{s}(1, \ldots ., n)
\end{array}\right) \quad 0 \\
& \text { I mod a chal } \\
& 5.12<155.12
\end{aligned}
$$

A.9 Ackley's Function

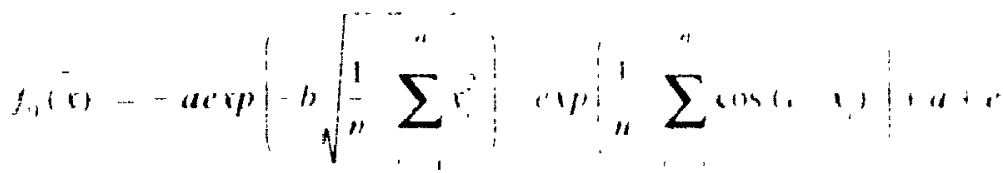

$$
\begin{aligned}
& a-20 ; b \quad 112,1 \quad 2 \pi \\
& 32.76 x-1,22.71,8 \\
& m m\left(y_{1}\right) \quad \text { 1.11....(1) } 11
\end{aligned}
$$

\section{A.10 Krolak's 100 City TSP}

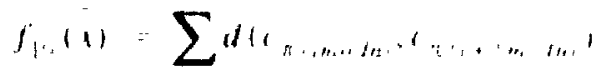

$$
\begin{aligned}
& 5.12 \cdot \div 5.12 \\
& \text { manly } 212 \times 5
\end{aligned}
$$


A.11 Low Autocorrelation Binary Sequences

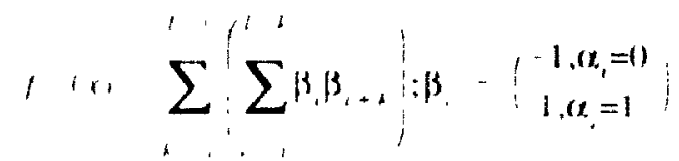

A.12 Hamming Distance to $0^{\prime}$

$$
\begin{aligned}
& f_{1:}(a) \cdot \sum_{i=1}\left(x_{1}\right. \\
& m i n(1 / 1)-f_{1}(1+\ldots . .10)-0
\end{aligned}
$$

A.13 Weierstrass-Mandelbrot Fractal Function

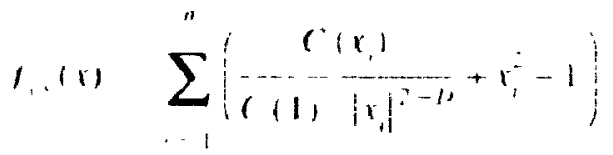

$$
\begin{aligned}
& (1 x)=\sum \frac{1-\cos (1) x)}{b^{\prime}(n)} \\
& 1 \leq l \leq \leq 2 ; b>1 \\
& 5.12 \leq 1,55.12
\end{aligned}
$$

A.14 Fully Deceptive Function

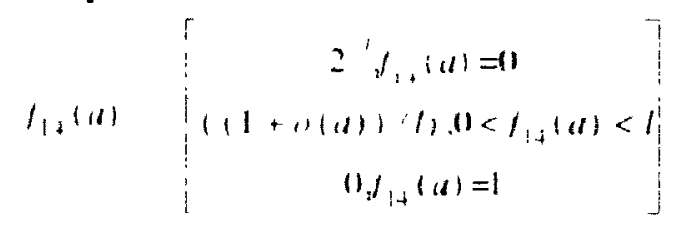

A.15 Weighted Sphere Model

$$
\begin{gathered}
1.11=\sum 11 \\
9.12<1,5912 \\
m 11+1, \quad i_{1}(19, \ldots 1)-11
\end{gathered}
$$




\section{A.16 Fletcher and Powell (fixed A,B matrices)}

$$
\begin{aligned}
& f_{i, 1} \sum_{i}(1, n)
\end{aligned}
$$

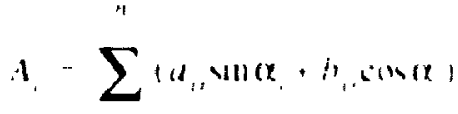

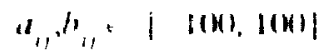

$$
\begin{aligned}
& (t, \cdot|-\pi, \pi|
\end{aligned}
$$

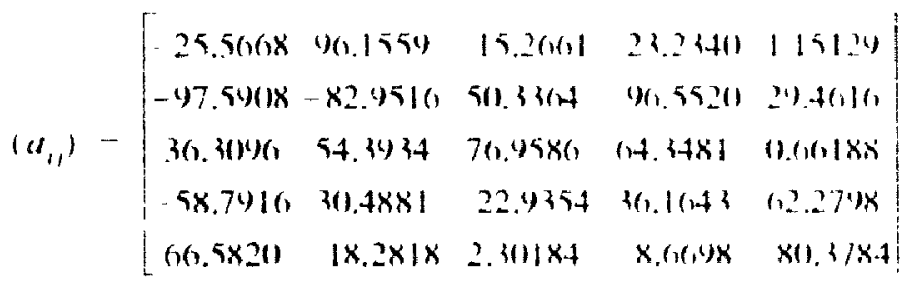

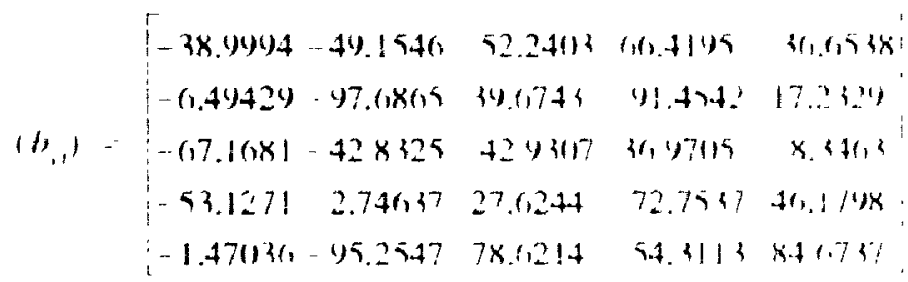

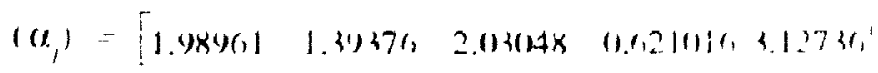


A.17 Fletcher and Powell (with random A,B matrices)

$$
\begin{aligned}
& \text { (.. (1) } \sum\left(4,-b_{1}\right)^{2} \\
& \text { A. }-\sum\left(a_{1}, \sin \alpha_{l}+b_{1,} \cos \alpha_{1}\right) \\
& \text { bi, } \quad \sum\left(a_{1, j} \sin x_{j}+b_{, j} \cos x\right) \\
& u_{4}, b_{1,}+|\cdot|(x), f(x) \mid \\
& a, \epsilon|-\pi, \pi|
\end{aligned}
$$

\begin{tabular}{|c|c|c|c|c|c|}
\hline $\mathbf{i}$ & \multicolumn{4}{|c|}{$\mathbf{A}(\mathbf{i})$} & $c_{i}$ \\
\hline 1 & 4 & 4 & 4 & 4 & 0.1 \\
\hline 2 & 1 & 1 & 1 & 1 & 0.2 \\
\hline 3 & 8 & 8 & 8 & 8 & 02 \\
\hline 4 & 6 & 6 & 6 & 6 & 0.4 \\
\hline 5 & 3 & 7 & 3 & 7 & 0.4 \\
\hline
\end{tabular}

In llis function, the $a_{i j}$ and $b_{i j}$ matrices are allowed to vary randomly.

\section{A.18 Shekel-5}

$$
\begin{gathered}
f_{1 \times}(x)=-\sum_{i=1}^{m} \frac{1}{(1-A(i))(x-A(i))^{2}+c_{i}} \\
n-4 ; m=5 \\
n \leq 1 \leq 10
\end{gathered}
$$

Table 14 Coefficients "or $f_{1 x}$ 


\section{A.19 Shekel-7}

$$
\begin{aligned}
& i_{1,4}(x)=-\sum \frac{1}{(x-1(i))(1, A(i))^{2}, \ldots} \\
& n-4: m \\
& 11<1,811
\end{aligned}
$$

\begin{tabular}{|c|c|c|c|c|c|}
\hline i & \multicolumn{4}{|c|}{$\mathbf{A}(\mathbf{i})$} & $c_{i}$ \\
\hline 1 & 4 & 4 & 4 & 4 & 0.1 \\
\hline 2 & 1 & 1 & 1 & 1 & 0.2 \\
\hline 3 & 8 & 8 & 8 & 8 & 0.2 \\
\hline 4 & 6 & 6 & 6 & 6 & 0.4 \\
\hline 5 & 3 & 7 & 3 & 7 & 0.4 \\
\hline 6 & 2 & 9 & 2 & 9 & 0.6 \\
\hline 7 & 5 & 5 & 3 & 3 & 0.3 \\
\hline
\end{tabular}

Table 15 Coefficients for $f_{14}$

$$
m(n)\left(j_{1,}\right)-f_{1,}(1, \ldots(1)) \quad 0
$$




\section{A.20 Shekel-10}

$$
\begin{gathered}
f_{i n}(x)-\sum_{1}^{m} \frac{1}{(x-A(t))(x-A(i))^{2}+1,} \\
n=4 ; m=10 \\
(1<1, \leq 11
\end{gathered}
$$

\begin{tabular}{|c|c|c|c|c|c|}
\hline$i$ & \multicolumn{4}{|c|}{$A(i)$} & $c_{\mathbf{i}}$ \\
\hline 1 & 4 & 4 & 4 & 4 & 0.1 \\
\hline 2 & 1 & 1 & 1 & 1 & 0.2 \\
\hline 3 & 8 & 8 & 8 & 8 & 0.2 \\
\hline 4 & 6 & 6 & 6 & 6 & 0.4 \\
\hline 5 & 3 & 7 & 3 & 7 & 0.4 \\
\hline 6 & 2 & 9 & 2 & 9 & 0.6 \\
\hline 7 & 5 & 5 & 3 & 3 & 0.3 \\
\hline 8 & 8 & 1 & 8 & 1 & 0.7 \\
\hline 9 & 6 & 2 & 6 & 2 & 0.5 \\
\hline 10 & 7 & 3.6 & 7 & 3.6 & 0.5 \\
\hline
\end{tabular}

Table 16 Coefficients for $\boldsymbol{f}_{\mathbf{2 0}}$

$$
\left.m(n) f_{Y n}\right)=f_{2}(1) \ldots(n)=0
$$




\section{A.21 Griewank-2}

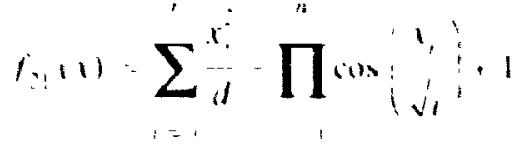

$$
\begin{aligned}
& d=2(n): n=2 \\
& \min \left(f_{21}\right)-f_{21}(0 \ldots . .01) \quad 0 \\
& -l(x) .0 \leq 1, \leq l(10) .0
\end{aligned}
$$

\section{A.22 Griewank-10}

$$
\begin{aligned}
& f_{2}(i)=\sum \frac{i}{d}-\prod \cos \left(\begin{array}{l}
1 \\
f_{1}
\end{array}\right)+1 \\
& d=4(k n): n \cdot 11 \\
& \left.\min \left(f_{2 .}\right)=f_{2,}(1) \ldots(1)\right) \quad 0 \\
& -\cos (x) .10 \leq x<\cos (x) .1)
\end{aligned}
$$

\section{A.23 Galar}

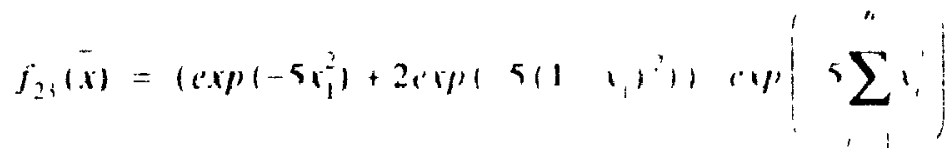

$$
\begin{aligned}
& .5 .10<8,-511
\end{aligned}
$$




\section{A.24 Kowalik}

$$
\begin{aligned}
& \left.f_{14} n \quad \sum \sum_{a}^{1} \frac{a_{1}\left(b^{2}+b_{1} x_{2}\right)}{b^{2}+b_{1}+r_{2}}\right)^{2} \\
& \text { 11- } 4 \\
& -5.11 \leq x, \leq 5.11
\end{aligned}
$$

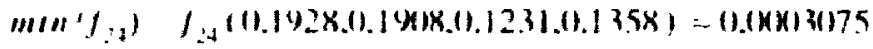

Table 17 Coefficients for $t_{24}$

\begin{tabular}{|l|l|l|}
\hline \multicolumn{1}{|c|}{$\mathbf{1}$} & \multicolumn{1}{|c|}{$\mathbf{a}_{\mathbf{i}}$} & \multicolumn{1}{c|}{$\mathbf{1}^{1} \mathrm{~b}_{\mathbf{i}}$} \\
\hline 1 & 0.1957 & 0.25 \\
\hline 2 & 0.1947 & 0.5 \\
\hline 3 & 0.1735 & 1 \\
\hline 4 & 0.1600 & 2 \\
\hline 5 & 0.0844 & 4 \\
\hline 6 & 0.0627 & 6 \\
\hline$I$ & 0.0456 & 8 \\
\hline 8 & 0.0342 & 10 \\
\hline 9 & 0.0323 & 12 \\
\hline 10 & 0.0235 & 14 \\
\hline 11 & 0.0246 & 16 \\
\hline
\end{tabular}




\section{Appendix B Results}

This appendix contains the results of using the adaptive unifom crossuct operater in lle" domain of function minimization. The definitions of the functions in the kest |xed uned ill this study can be found in Appendix A. Three graphs for each function are induled. Iach chart is a plot of the fitness of the best solution in the population after a given mumber of trials plotted against the number of trials. The first graph shows the results of the |xest al trial' fitness for the standard uniform crossover operator. The xecond praph shous llic results of the best-at-trial fitness for the adaptive unifom crossover oprotalot. Ihe final graph shows the difference between the best-at-trial fitness for the standand untom crossover operator and the adaptive uniform crossover operator. Therefore, in the third graph, when the difference values fall below zero, the adaptive run is superior to the non-adaptive run.

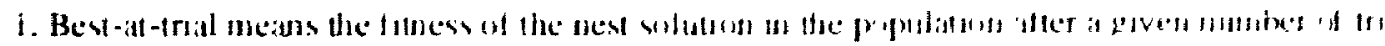
als. 


\section{B.1 Results}

The adlaptive uniform crossover operator was tested against all functions in the function lest bed - $t_{1} 10 t_{24}$. The results were compared using equivalent genetic algorithm search parameters to the uniform crossover operator. A modified version of Grefenstette's genetic al gorithm experimental tool GENESIS ${ }^{1}$ was used for all experimental runs. In ali genetic algorithm runs, the same basic parameters were chosen. These are shown in Table 18:

Table 18 Common experimental parameters

\begin{tabular}{|c|c|c|}
\hline Variable & Meaning & Value \\
\hline$p_{s}$ & Population size & 50 \\
\hline$P_{m}$ & Probability of mutation (per bit) & 0.001 \\
\hline$N_{\mathbf{\sigma}}$ & Number of experiments & 100 \\
\hline$N_{t}$ & Number of trials per experıment & 10.000 \\
\hline $\mathrm{S}$ & Selection mechanism & Roulette wheel \\
\hline Codıng & $\begin{array}{l}\text { How object variable is encoded in the bit } \\
\text { string }\end{array}$ & Gray coding \\
\hline 1 & $\begin{array}{l}\text { Lengtt of the object variable encoding part } \\
\text { of an individual. }\end{array}$ & $\begin{array}{l}1=32 n \text { in being the num. } \\
\text { ber of objective function } \\
\text { variables) }\end{array}$ \\
\hline
\end{tabular}

The crosisuver probability for the unifom crossover operator was set to 0.5 . The geometric form of the crossover exchange operator was used to decide whether bit-wise crossover was to take place and $P^{\prime}$ had the functional form: $P^{\prime}(x)=s / n: s=0, \ldots, n$. making the automata examples of the $D_{-R P}$ class of automata.

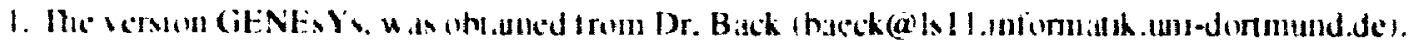


The values of the various automata probahilities are shom n in lable IU:

Table 19 Adaptive crossover operator parameters

\begin{tabular}{|c|c|c|}
\hline Variable & Meaning & Value \\
\hline$p_{\text {supenorReward }}$ & $\begin{array}{l}\text { Probability with which an automaton will be } \\
\text { rewarded if the offspring is fitter than the } \\
\text { parent. }\end{array}$ & 1.0 \\
\hline Pinteriorpenalty & $\begin{array}{l}\text { Probability with which an automaton will be } \\
\text { penalized if the offspring is less fit than the } \\
\text { parent. }\end{array}$ & 10 \\
\hline PsamePenalty $_{\text {sam }}$ & $\begin{array}{l}\text { Probability with which an automaton will be } \\
\text { penalized if the offspring is as tit but no fit- } \\
\text { ter than the parent. }\end{array}$ & 0.0 \\
\hline$\rho_{\text {sameReward }}$ & $\begin{array}{l}\text { Probability with which an automaton will be } \\
\text { rewarded if the offspring is as fit but no fitter } \\
\text { than the parent. }\end{array}$ & 00 \\
\hline Iolerance & $\begin{array}{l}\text { Floating point tolerance used to decide } \\
\text { whether an offspring is more or less fit than } \\
\text { its parent(s). }\end{array}$ & 00 \\
\hline
\end{tabular}

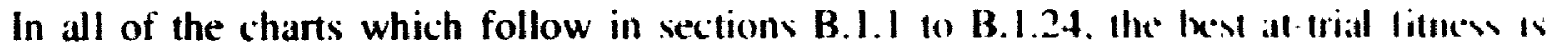
plotted. Other interesting statistics which were reviewed, hut not included due Io yalce constraints, were online and offline perfomance and population diversity. The $y$ ass represents the best-at-trial fitness and the $x$ axis the Irial number. Being funciun minimization problems, we expect the best-at-trial fitness on decrease with incteasult wial number. The ociasional increase in best-at-trial fitness for some functions is due lo lue stochastic nature of the roulette wheel reproduction strategy which all destloy $\left|k^{2}\right| x^{*} \mid$ solution in the population at any time. Generally. the inest-at-trial meessuce for the funchun test bed is monotone, decreasing in nature. 
Appendix B: Results

Page 164

B.1.1 Sphere Model

Mot of $f 1$ is Trial for non adaptive run

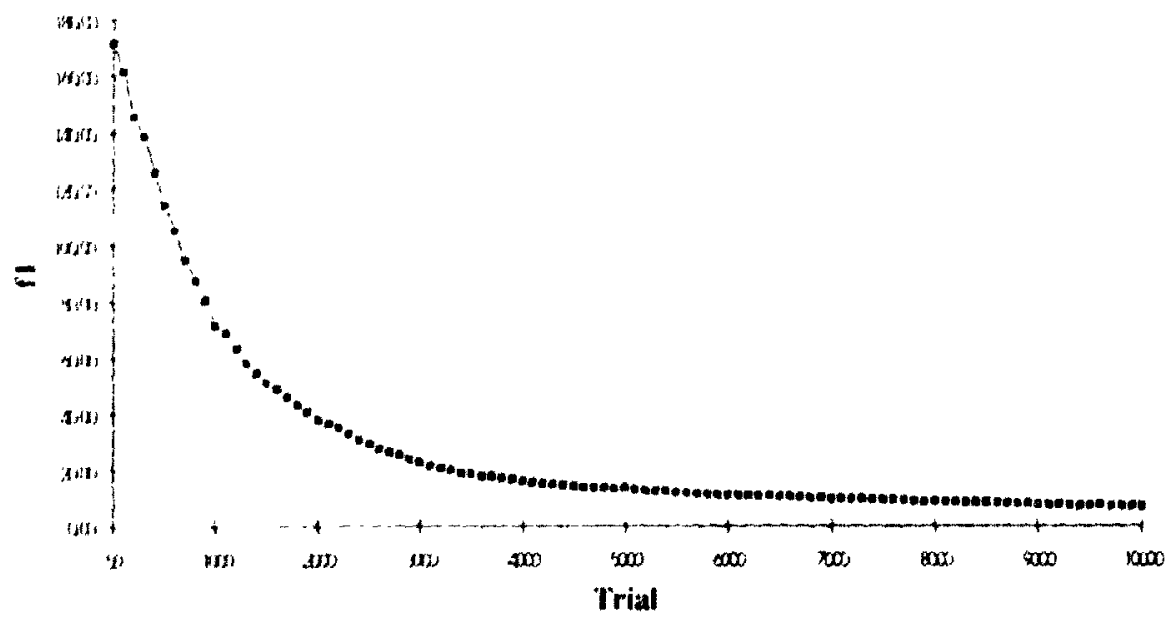

Figure 35 fl nun-adistive fitness

Mot of II versus Trial fur adaptive run

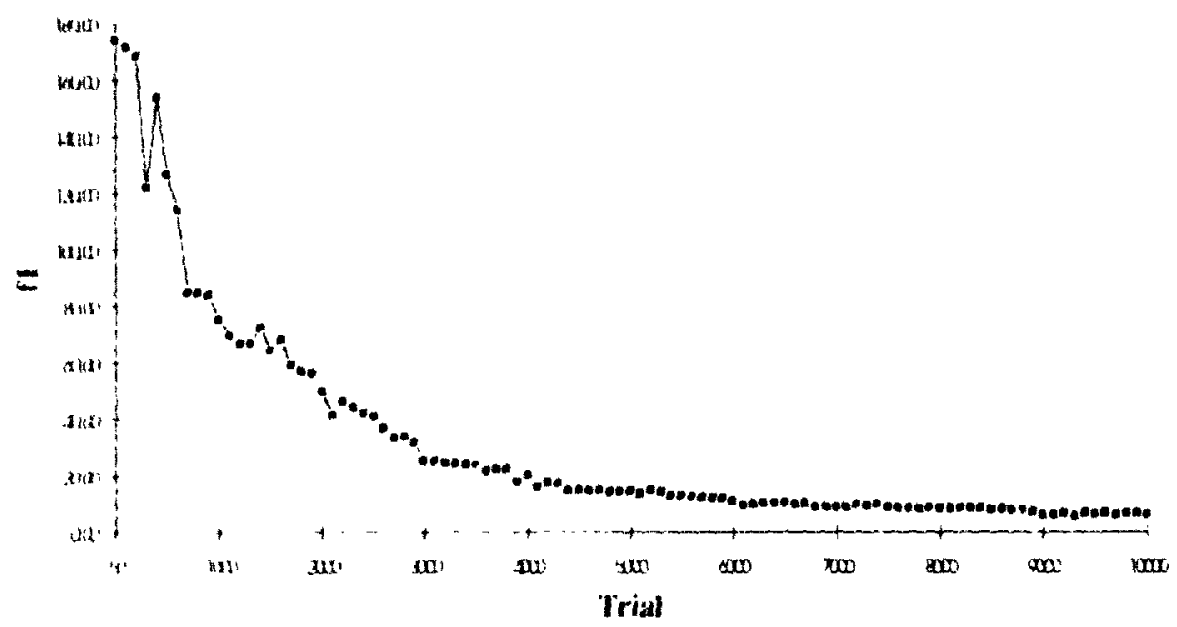

Figure $\mathbf{3 6} \mathrm{fI}$ adaptive fitness 
Mut of fl difference letween adaptise and non-ad:ıptive rums

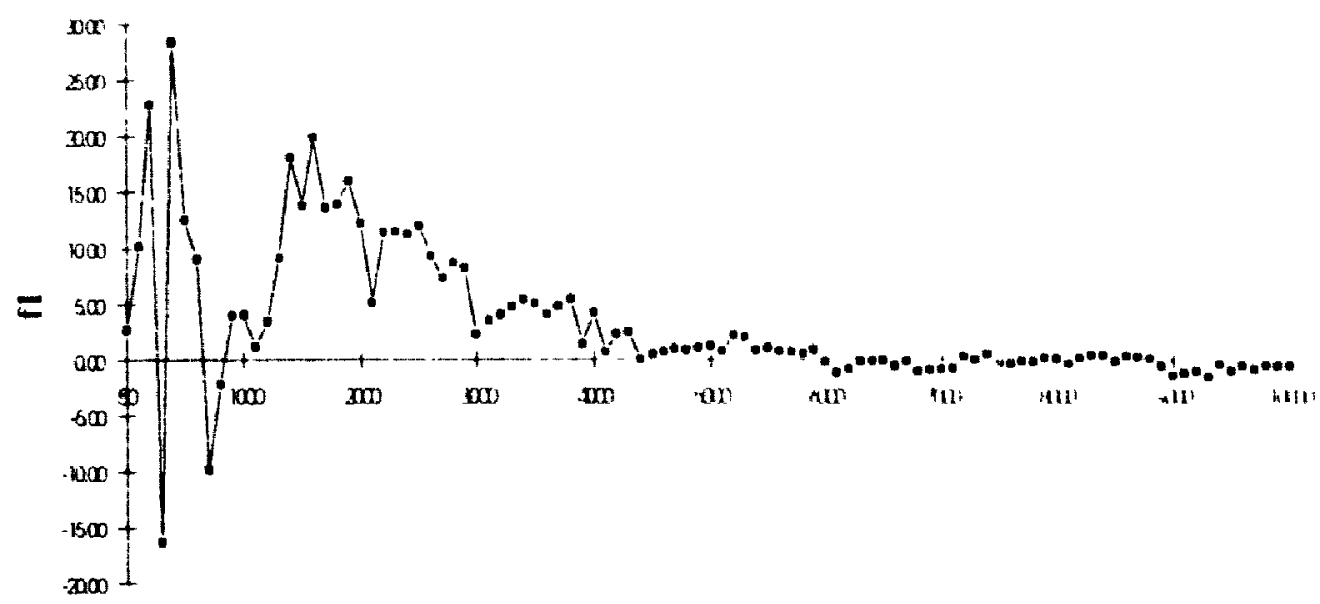

Trial

Figure 37 fl adaptive - non-adaptive comparison

\section{B.1.2 Generalized Rosenbrock's Function}

Phit of 2 versus Trial fur adaptive run

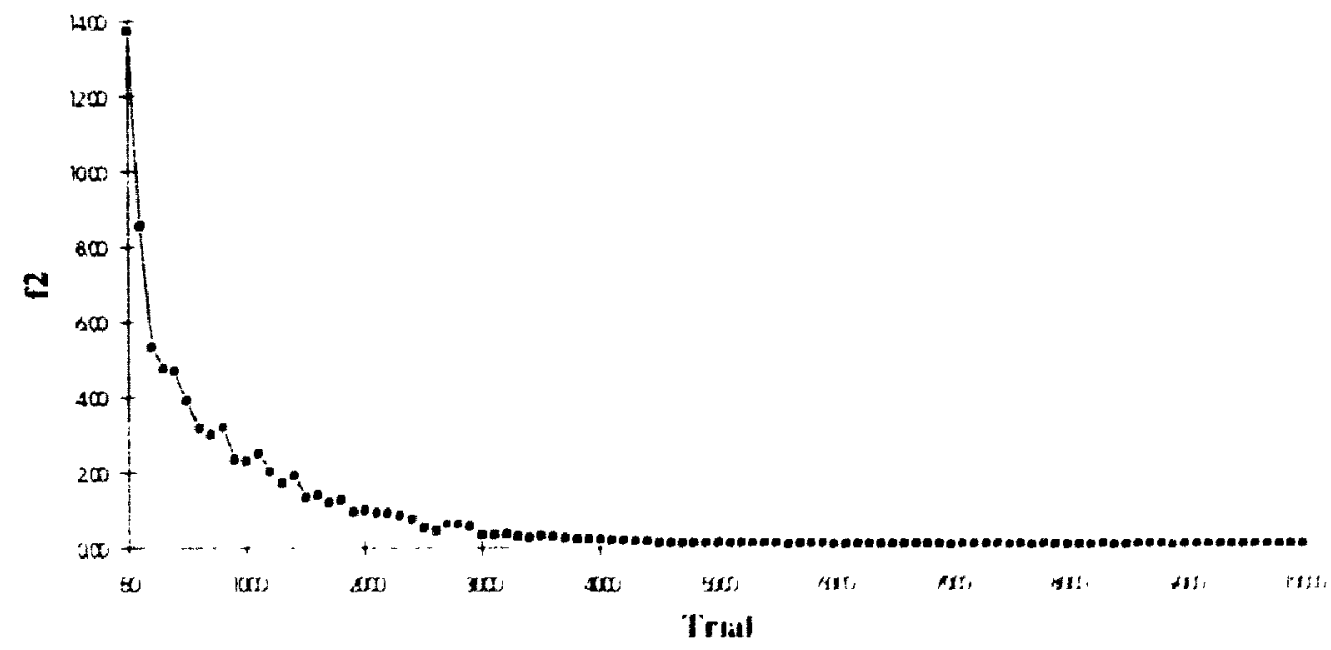

Figure 38 idaptive fitness 
Plot of 22 versus Trial for non-adaptive run

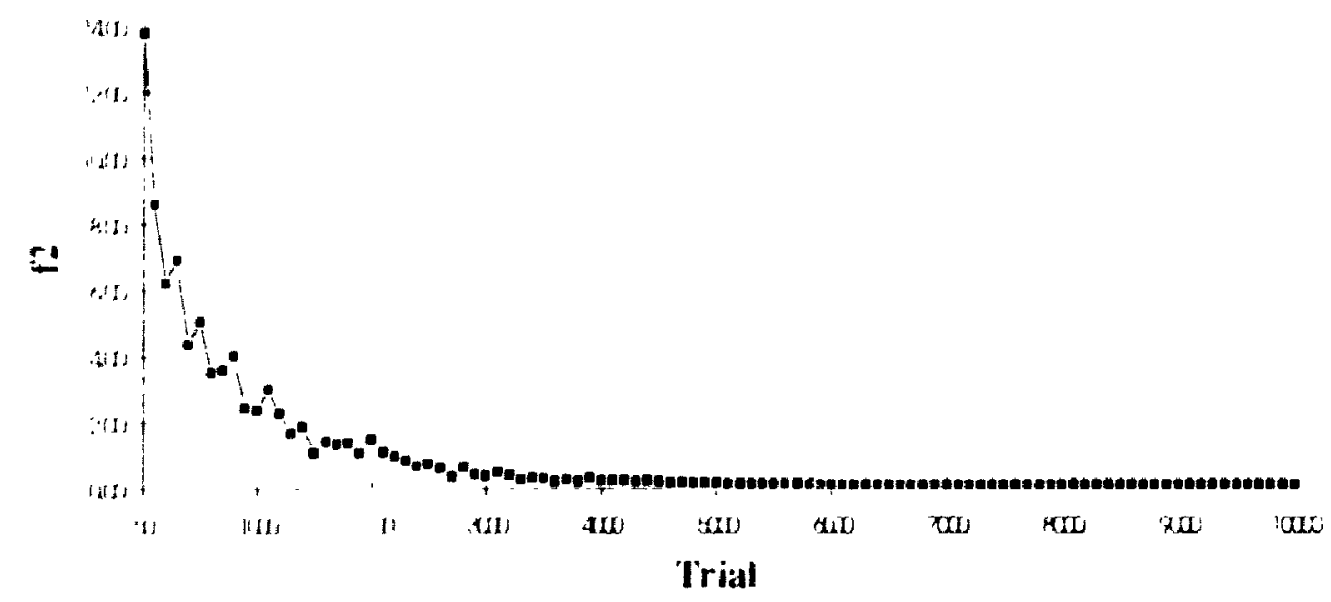

Figure 34 2 non-adaptive fitness

Blot of 12 difference bet ween adaptive and non-adaptive runs

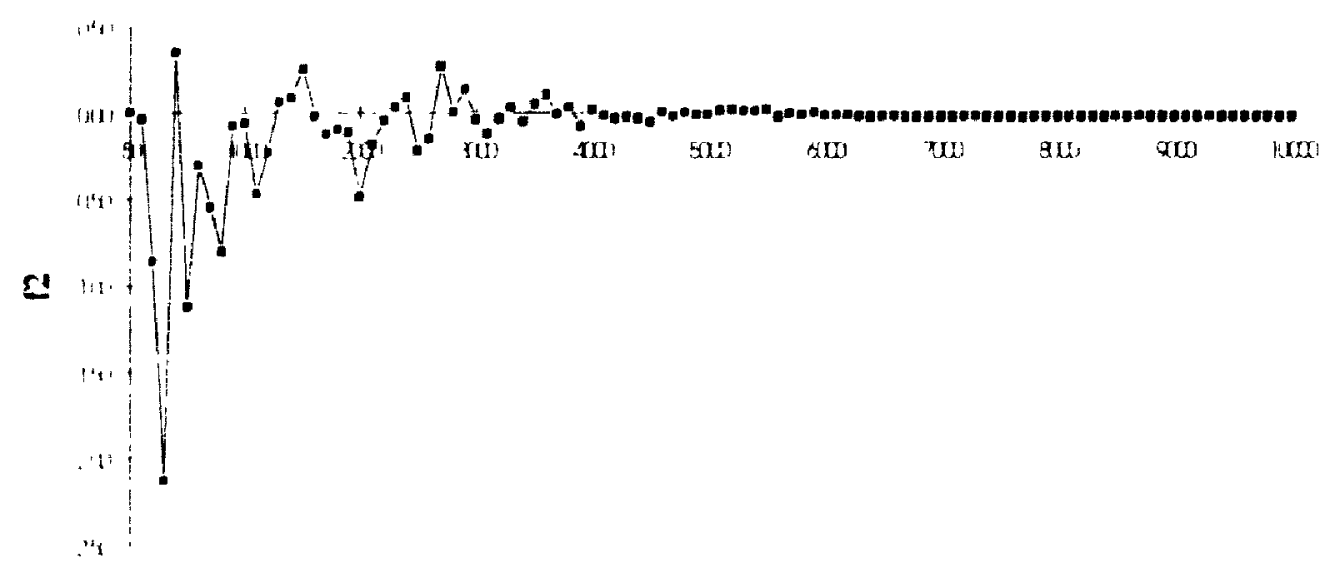

Trial

Figure 40 2 adaptiva - mon-adaptive comparison 


\section{B.1.3 Step Function}

Mot of fo leras Trial firr adaptice run

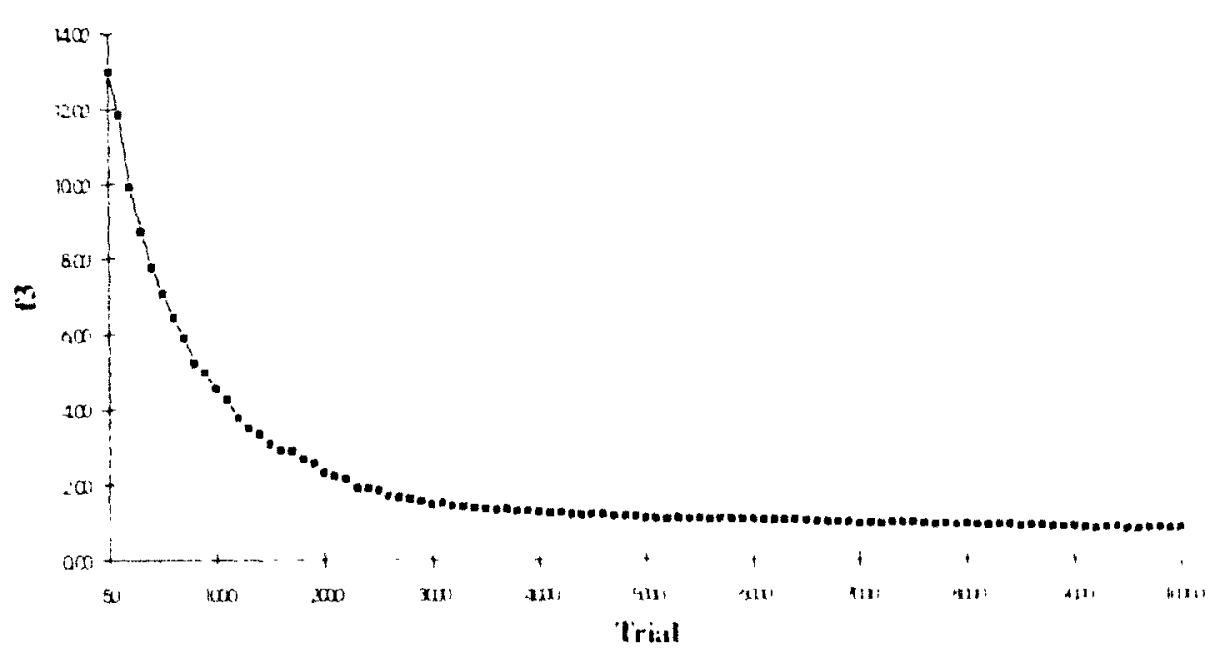

Figure $41 \mathrm{f3}$ adaptive fitness

Plot of fi3 versus Tial for nenl-adaptis e run

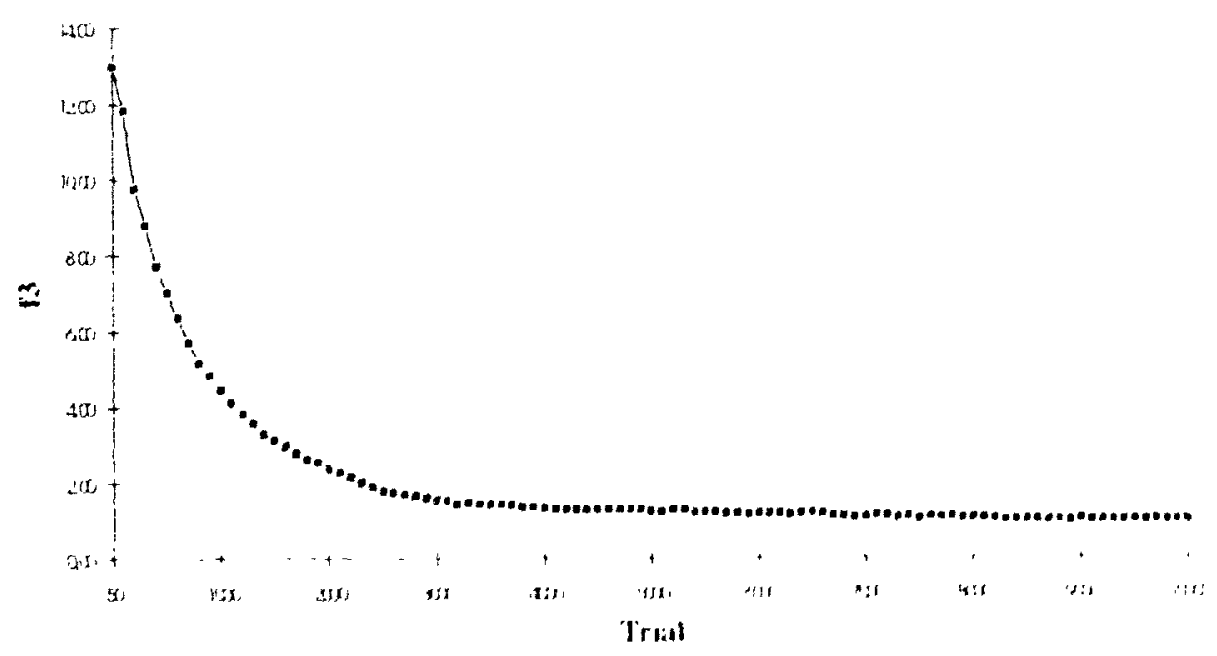

Figure 42 i3 non-adaptive fitness 
Plon af fis versus I'rial difference between adaptic and mon-adaptive runs

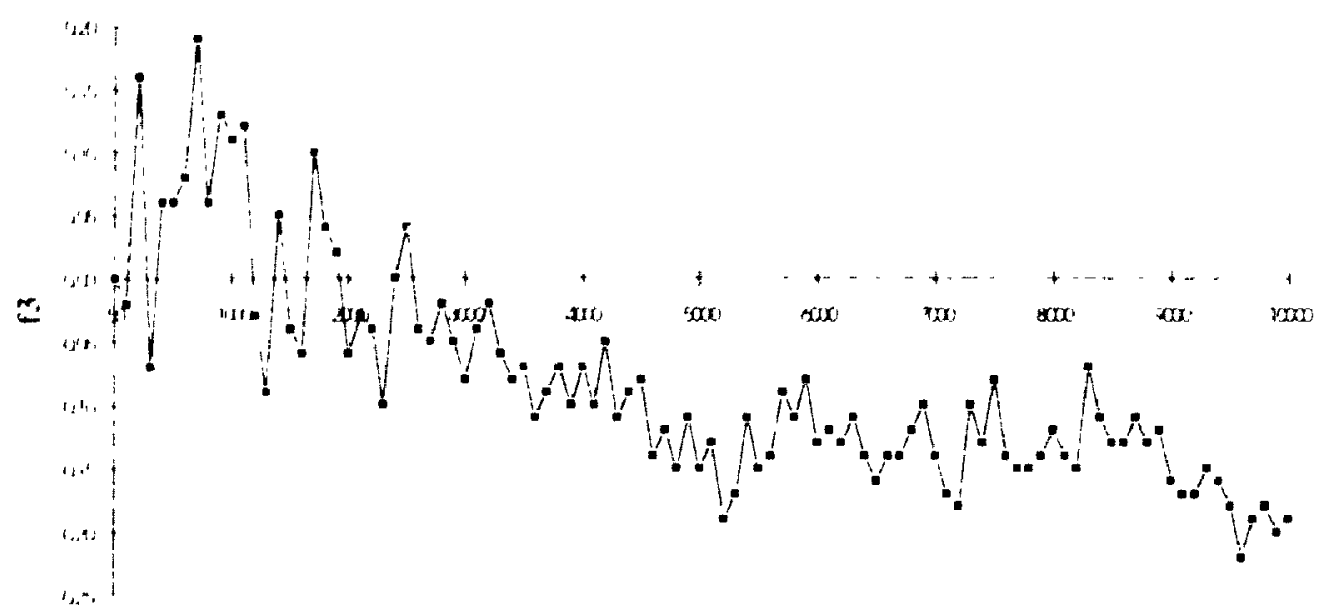

Trial

Figure 4.3 i3 adaptive - non-adaplive comparison

\section{B.1.4 Quartic Function with Noise}

Plut of ft versus Trial fur non-adaptive run

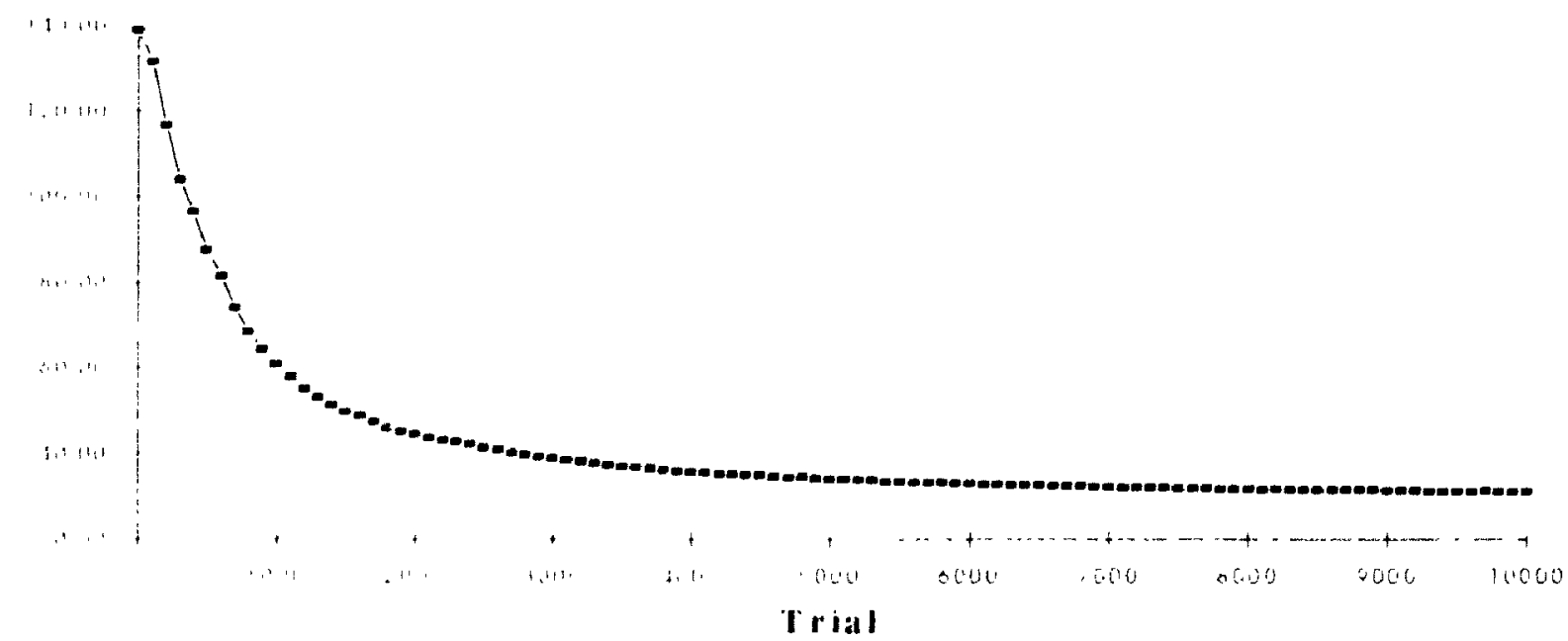

ligure if it mon-adaptive fitness 
Appendix H: Kesults

Plot of f versus T rial for adaptive run

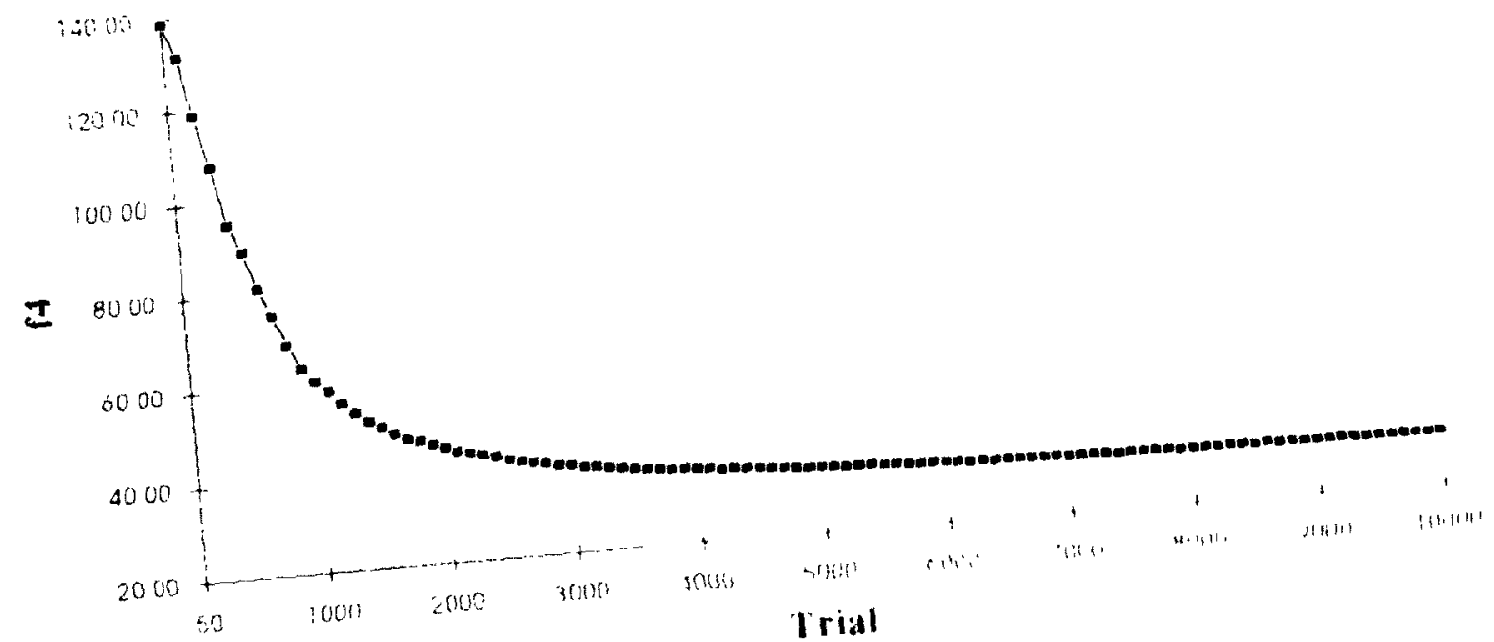

Figure 45 ft adaptive fitness

Plot of 4 varsus i rial difference betweel adaptive and nonadaptive runs

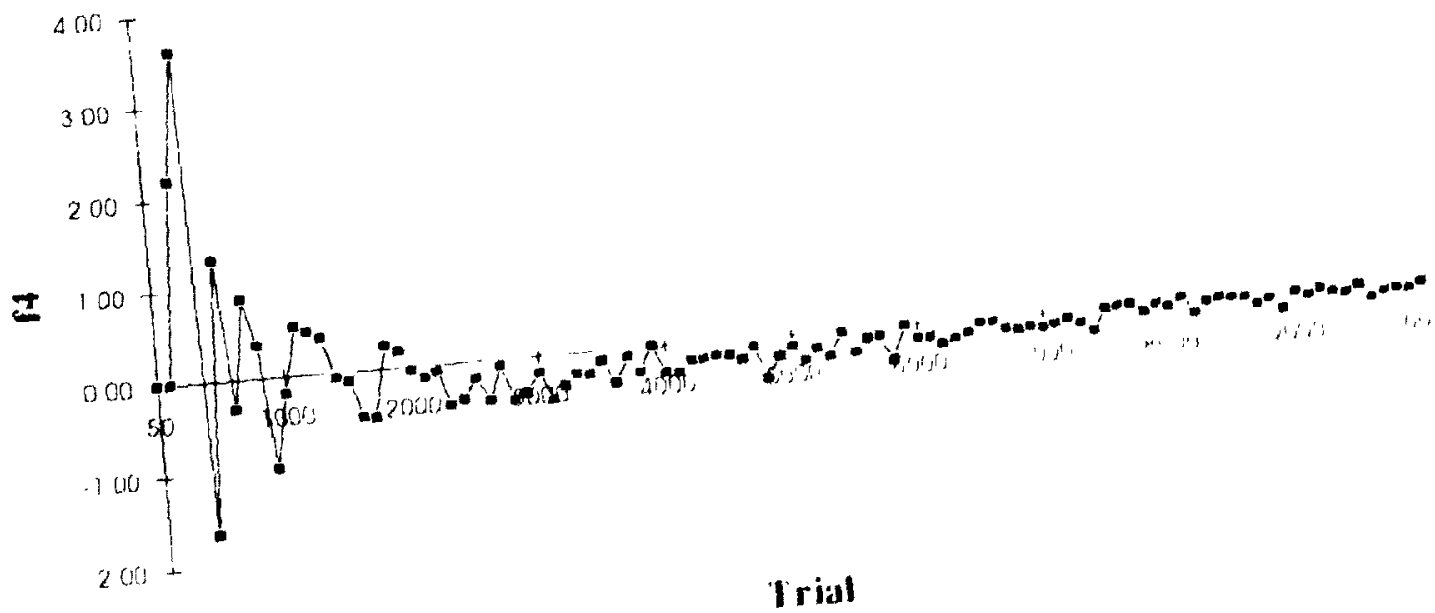

Figure 46 it adaptive-non-adaptive comparison 


\section{B.1.5 Shekel's Foxholes}

Phut of ts venus Trial for adaptive run

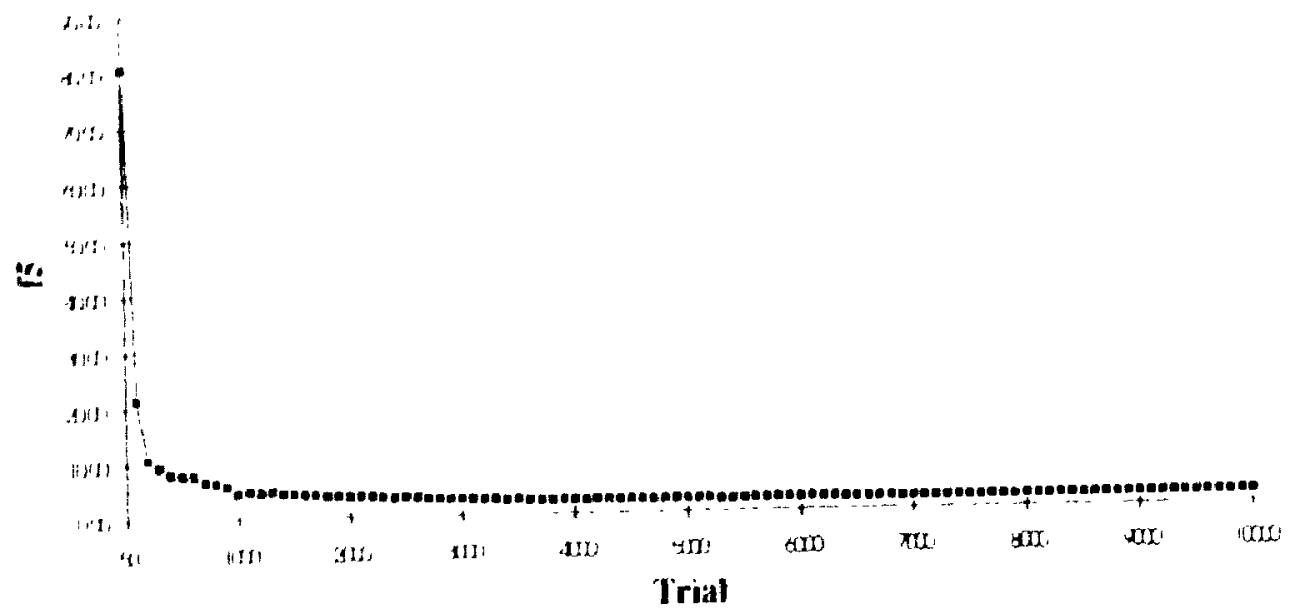

Figture 47 is adaptive fitness

Plat of t5 versus Irial for mon-adaptive run

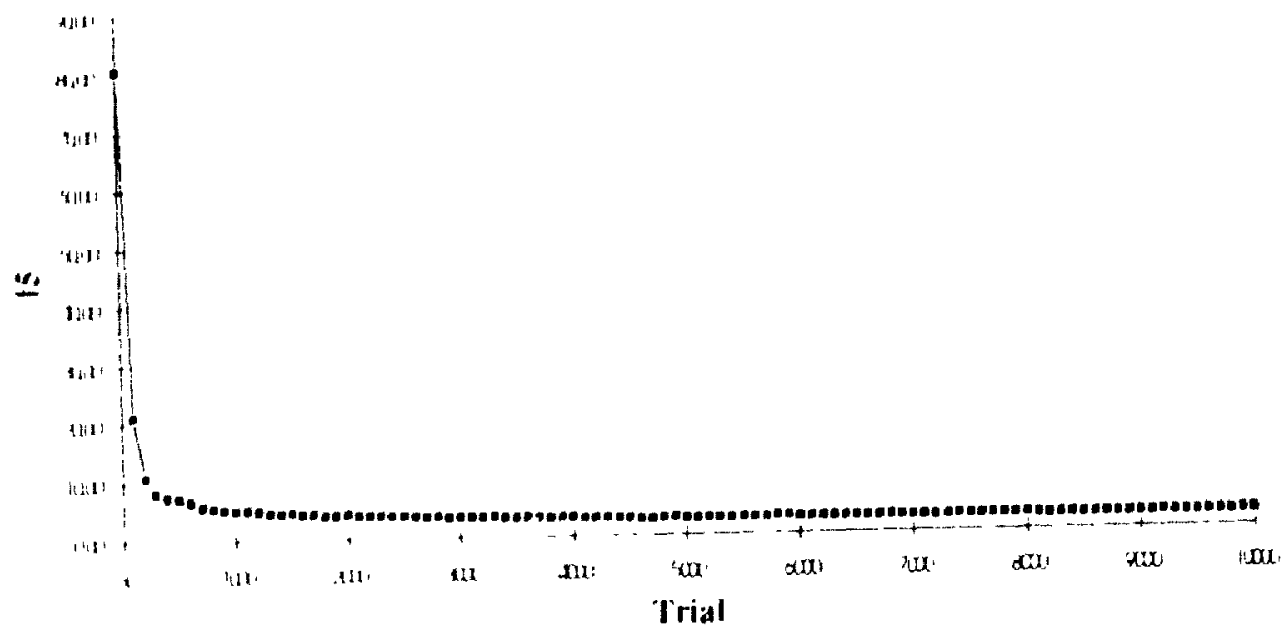

Figure 48 is mon-adaptive fitness 
Plot of f5 versus Trial difference betern adaptice and mon-adaplic e rums

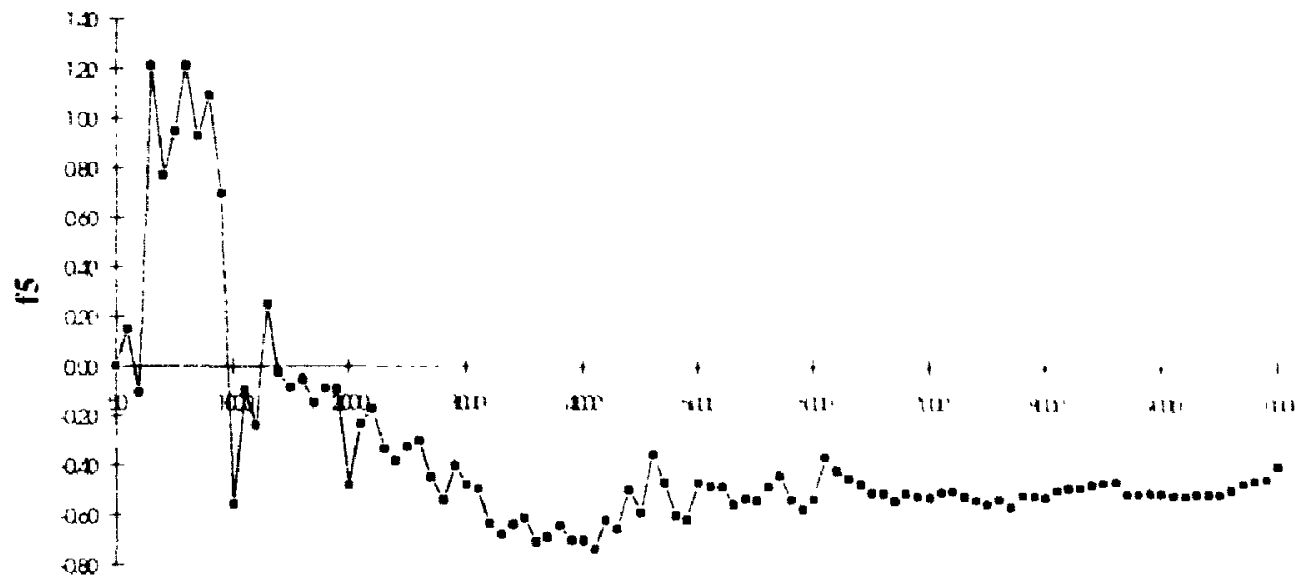

Trial

Figure 49 f5 adaptive - non-adaptive comparison

\section{B.1.6 Schefel's Function $\mathbf{1 . 2}$}

Plot of fo versus Trial for adaptive run

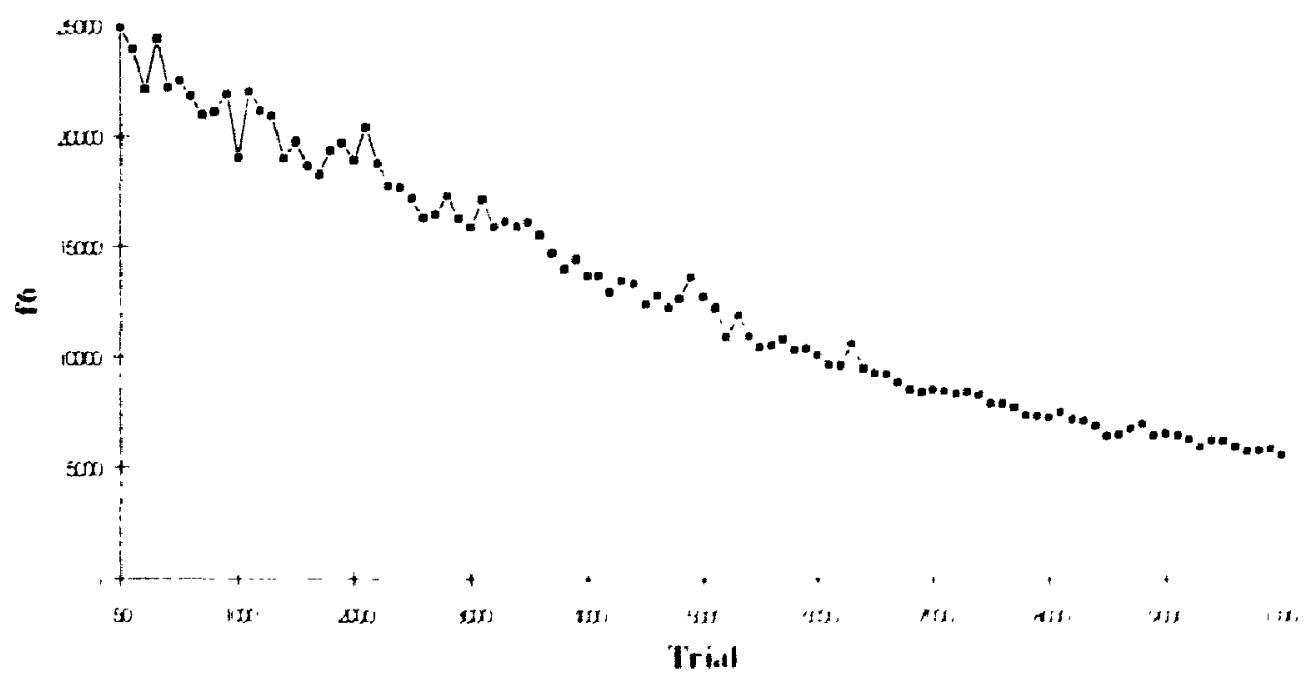

Figure 50 (6 adaptive fit ness 
Plent of fo versus 'Irial fur men-adaptive run

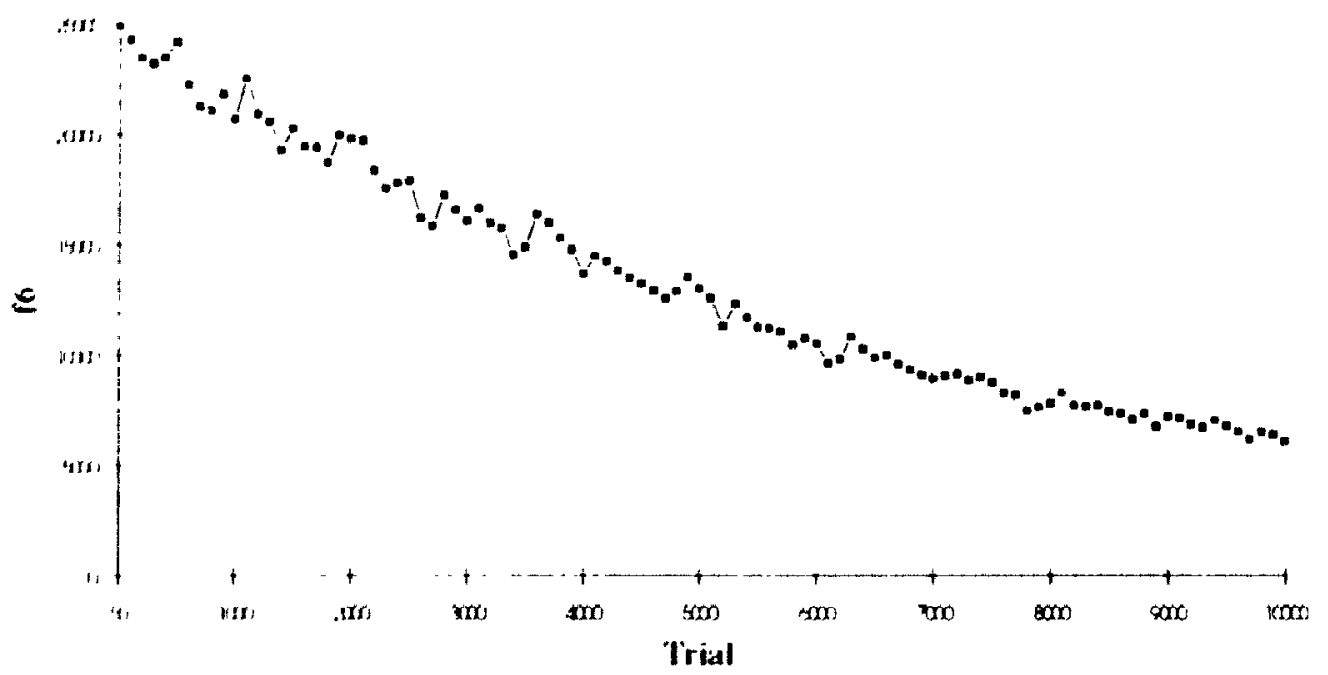

Figure 51 fo non-adaptive fitness

Plot of th sersus Traal difference between adaptive and mon-adaptive rurs

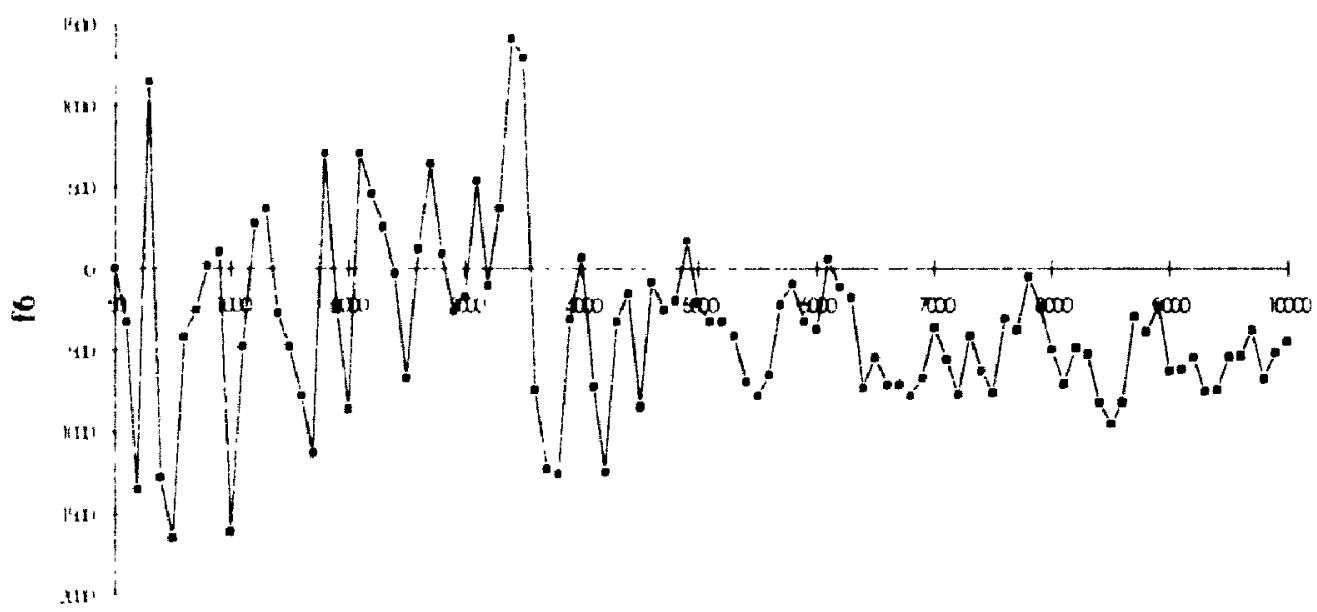

Trial

Figure 52 fo adaptive - non-adaptive comparison 


\section{B.1.7 Generalized Rastrigin's Function}

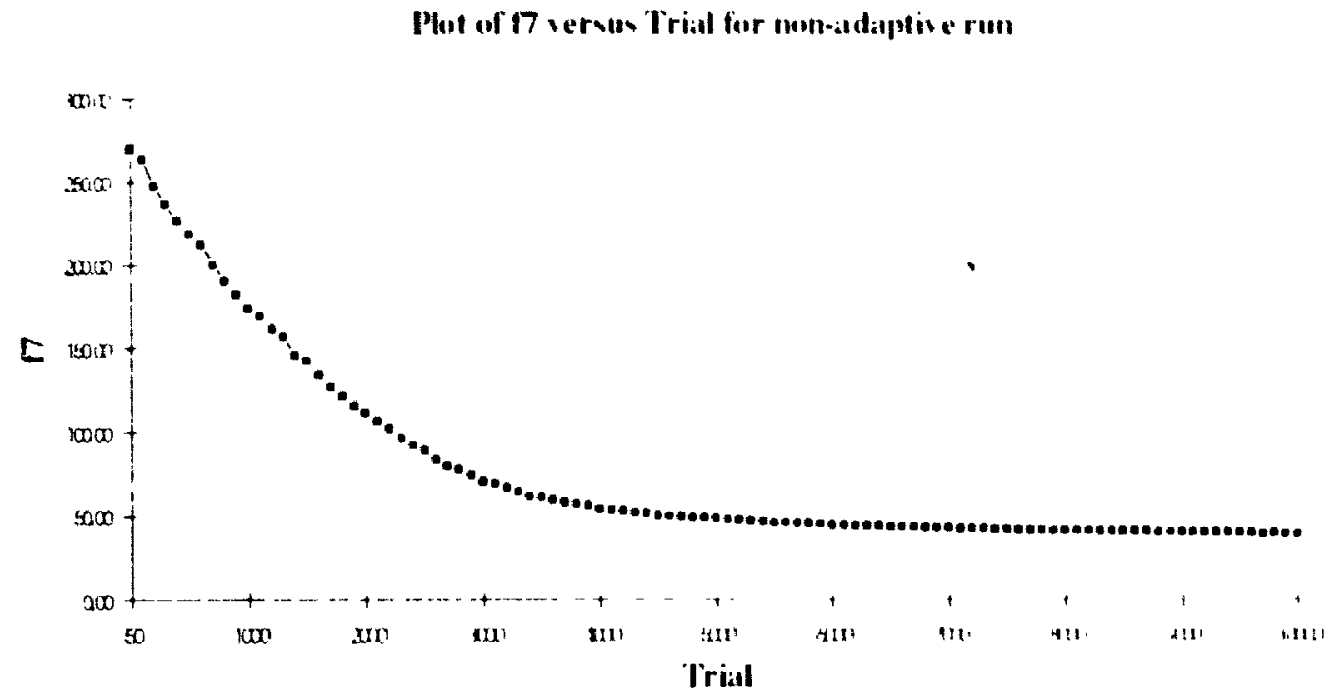

Figure 53 i non-adaptive fitness

Plot of 17 sersus Trial for adaptise rum

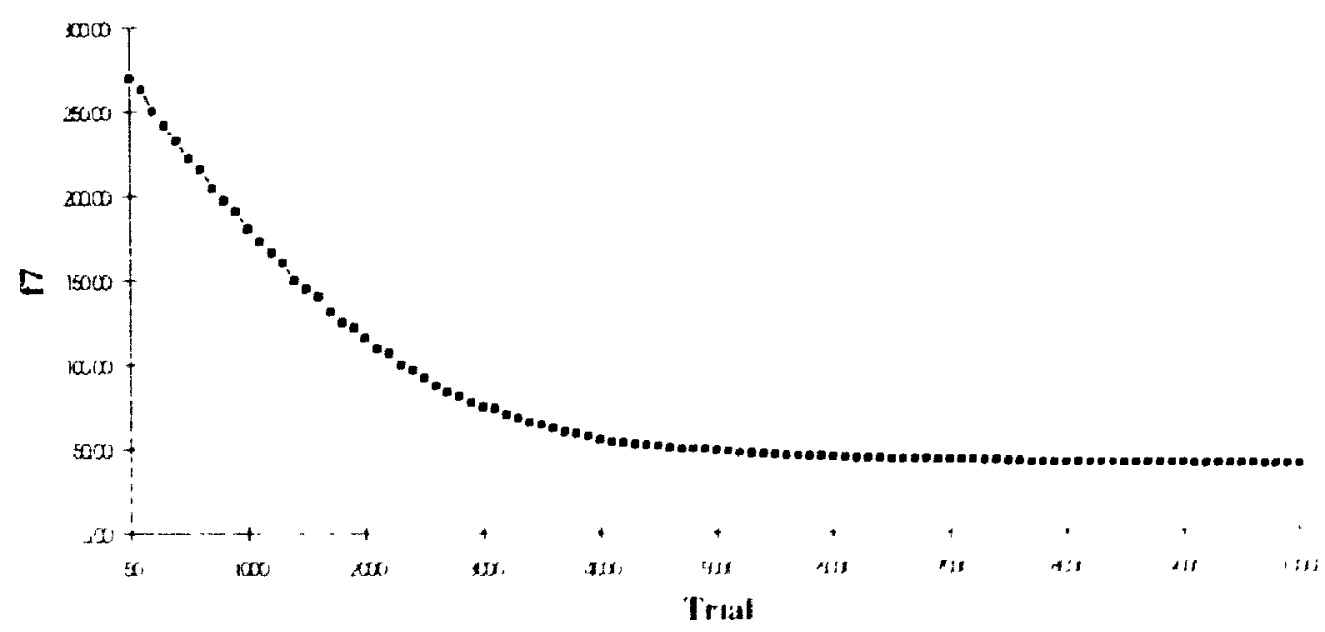

Figure 5417 adaptive fitness 
InIt of 77 versus Trial difference bet ween adaptive and mon-adaptive runs

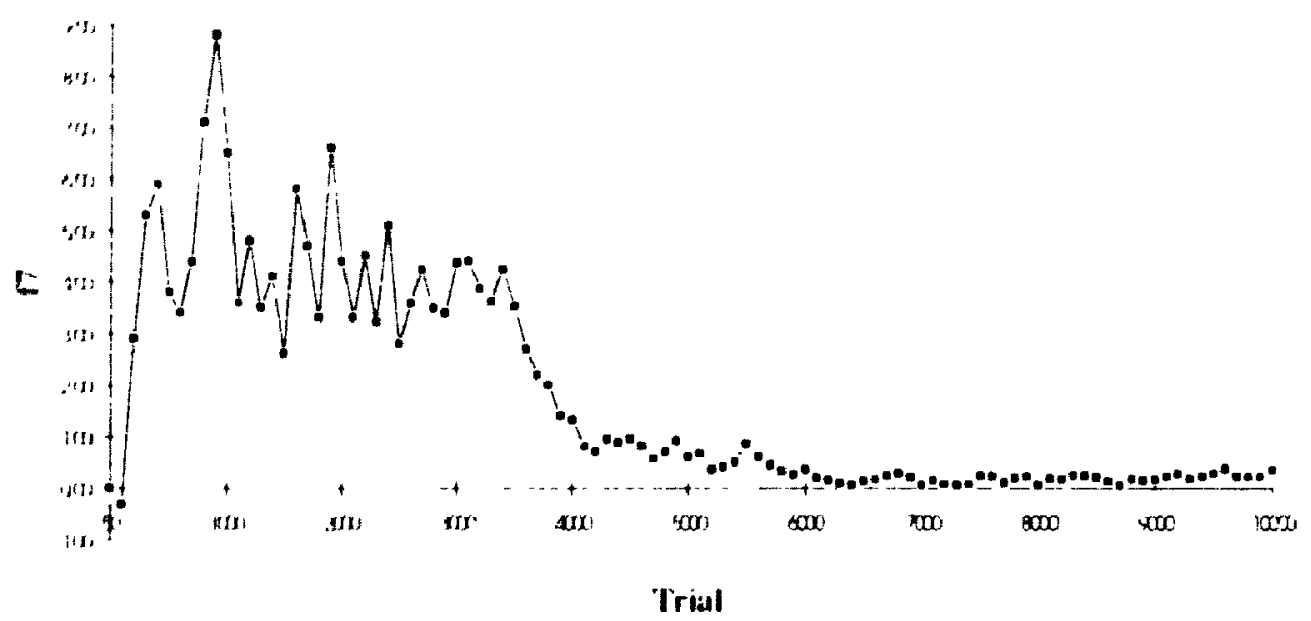

Figure $55 \quad 17$ adaplive - non-adaptive comparison

\section{B.1.8 Sphere Model, Changing Environment}

Plot of $\mathbf{X}$ versus Trial for non-adaptive run

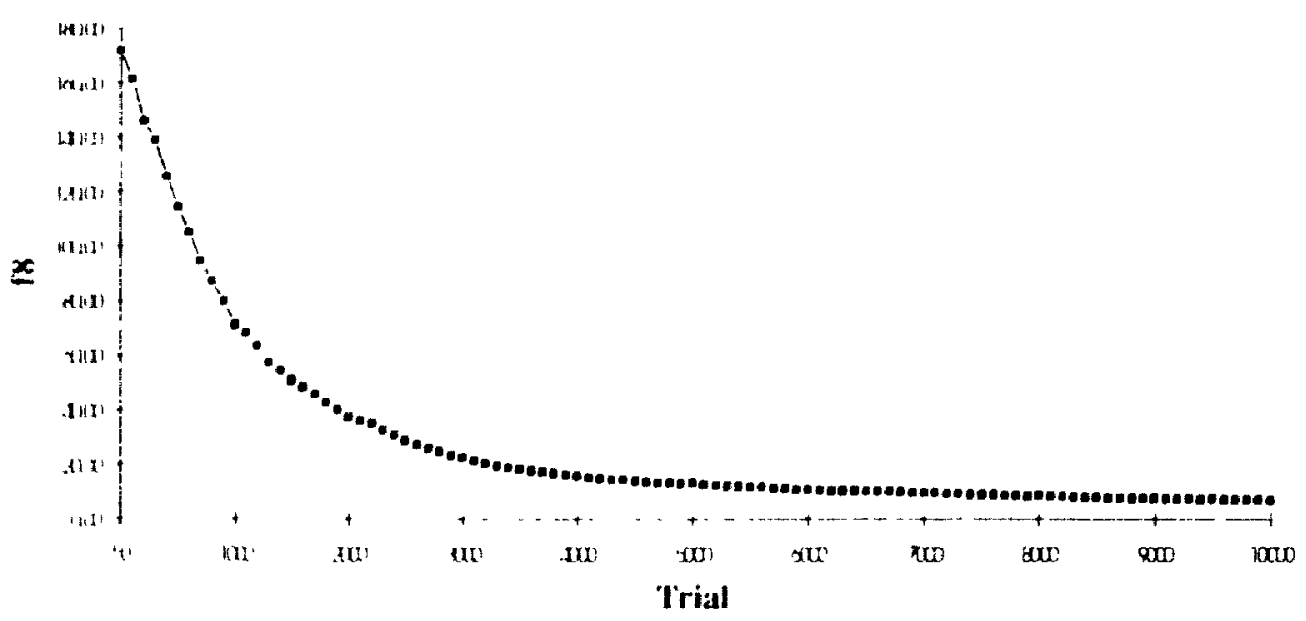

Figure 56 IX non-adaptive fitness 
Plut of fX versus 'Trial for adaplice run

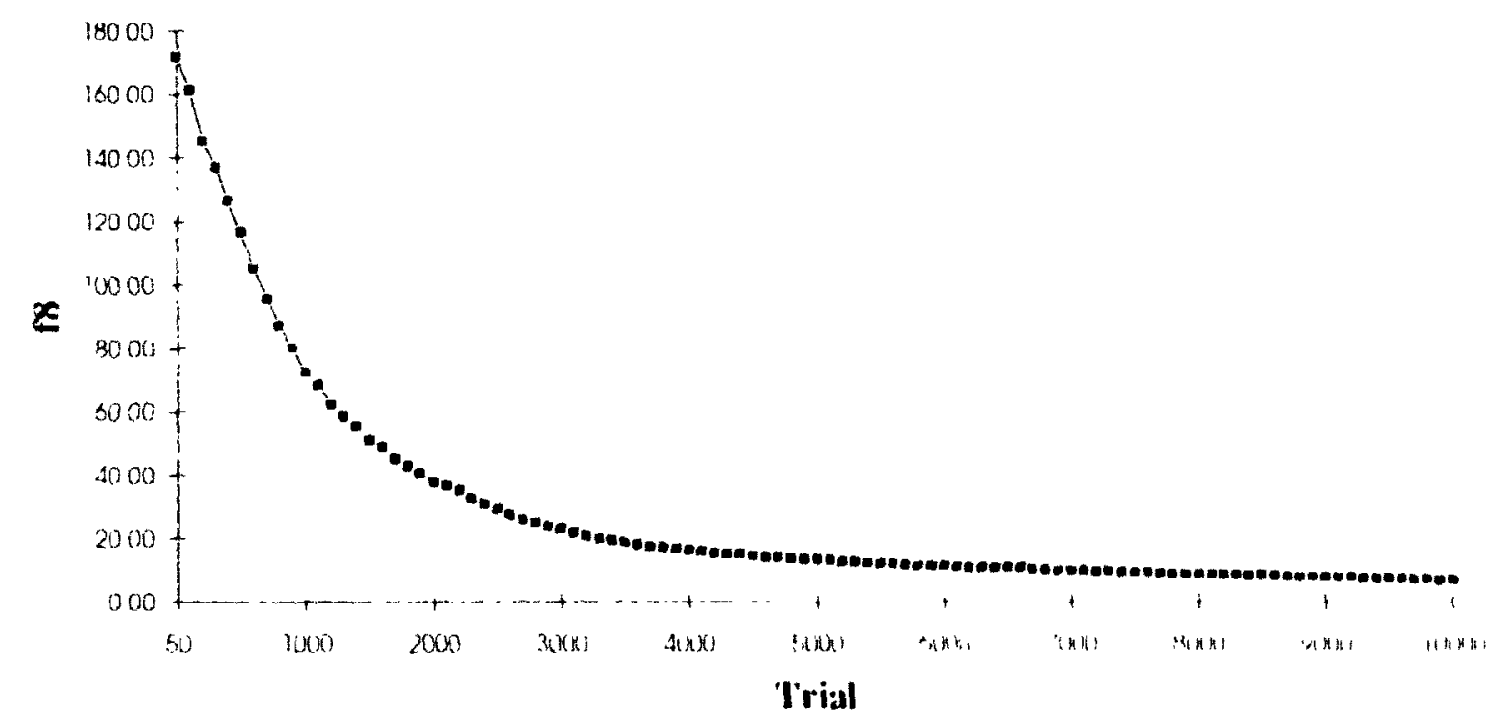

Figure 57 IX adaptive fitness

Plot of th versus Trial difference bet weel adaptive and non-adaphive runs

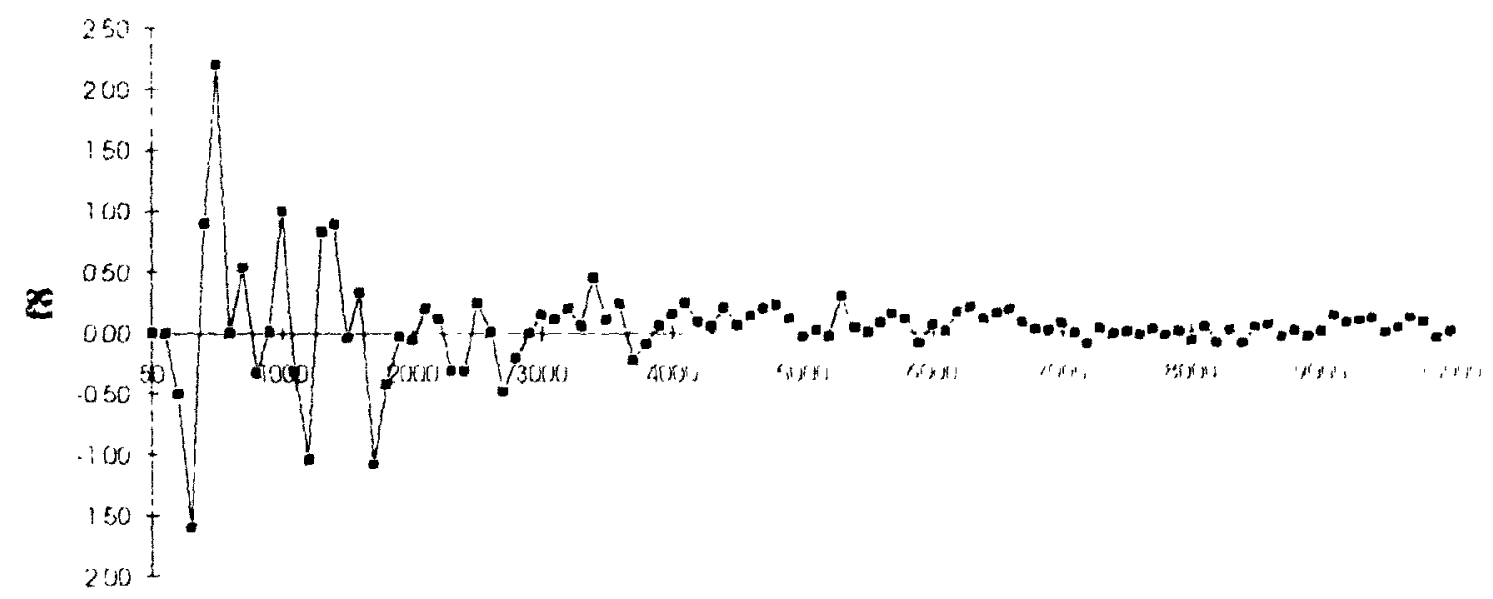

Trial

Figure 58 fX adaptive - non-adaptive comparison 


\section{B.1.9 Ackley's Function}

Mhet of 19 versus Trial for mon-adaptice run

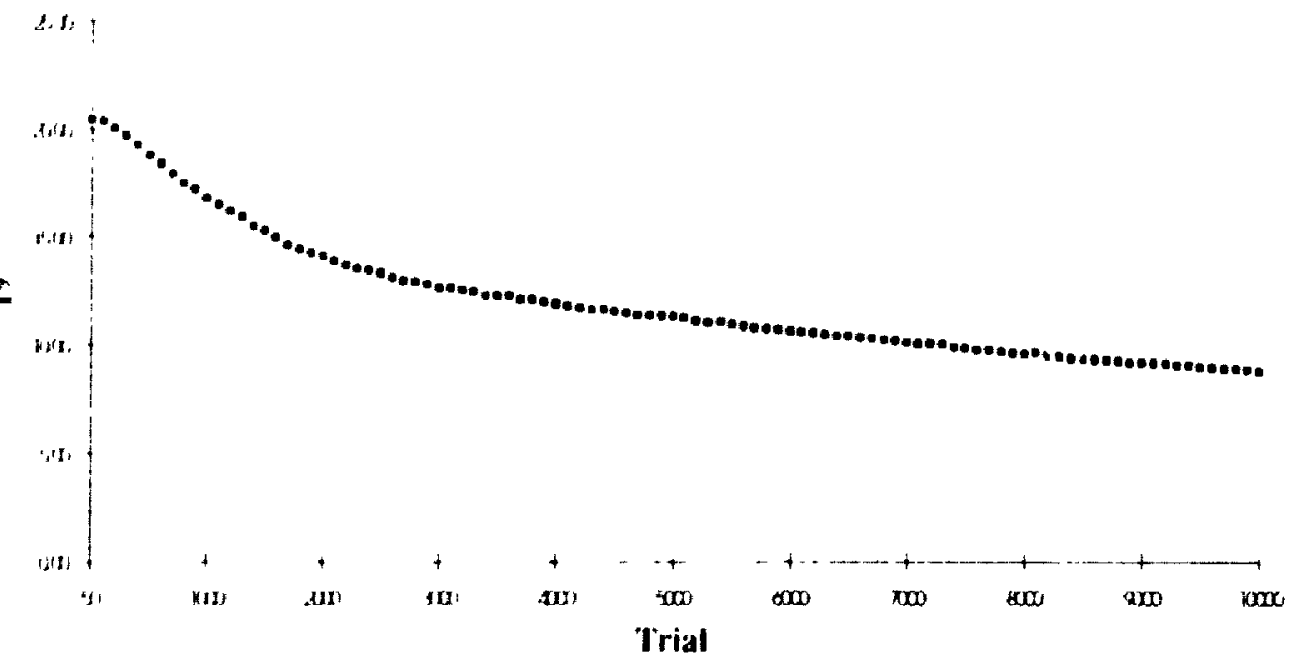

Figure 5y iv mon-adaptive fitness

Pot of t9 versus Trial for adaptive run

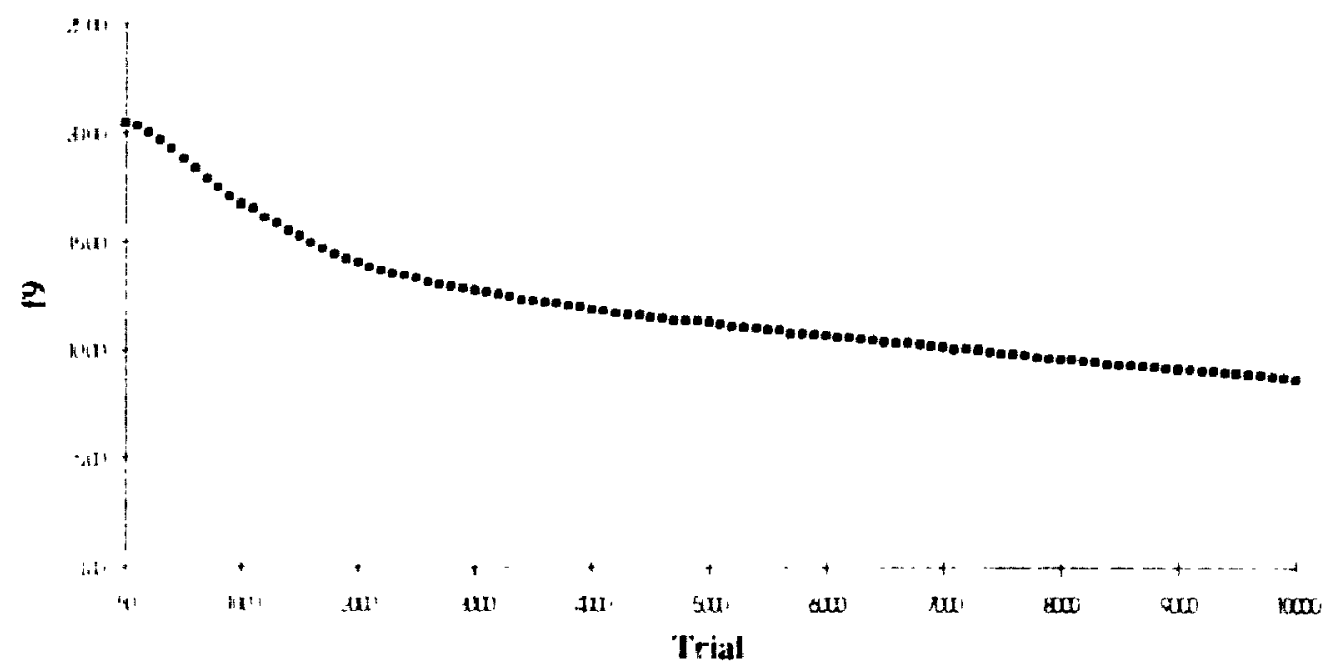

Figure (b) (Y) adaptive fitness 


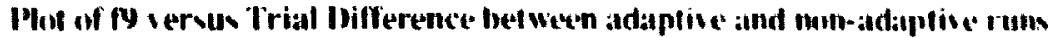

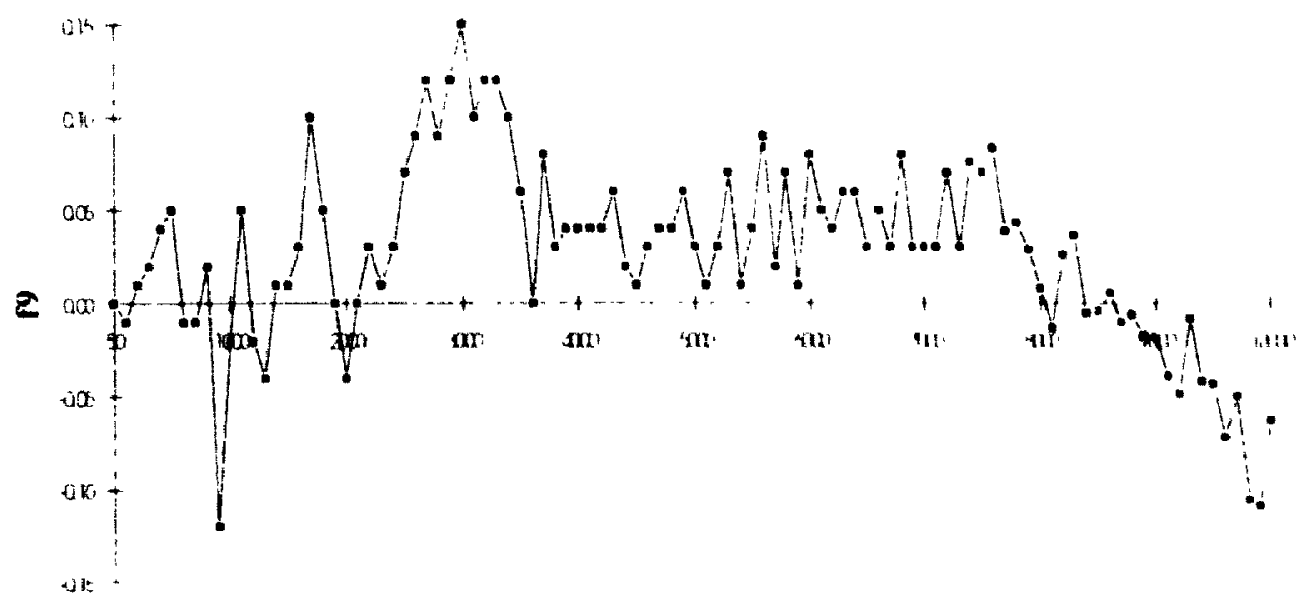

Trial

Figure 6I Y adaptive - non-adaptive comparison

\section{B.1.10 Krolak's 100 City TSP}

Plot of flo vervis Trial fur moll-adaptive ran

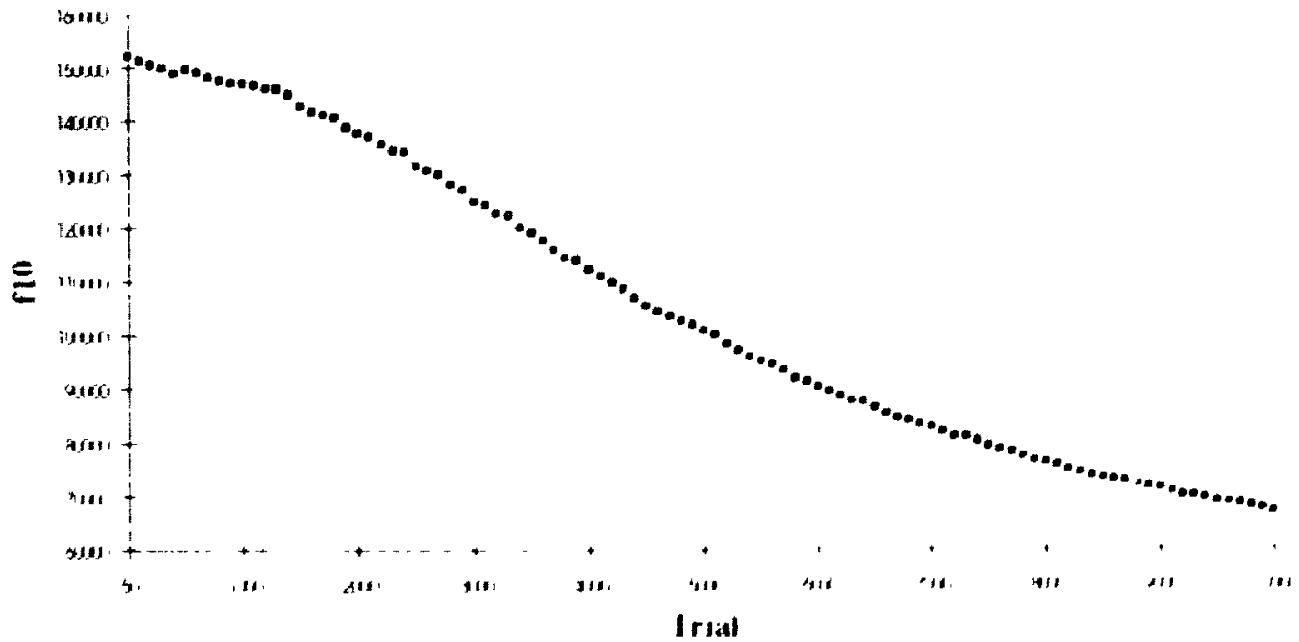

Figure 62 fill non-adaptive fitness 
Pof of fill verwas Trial for adaptis e run

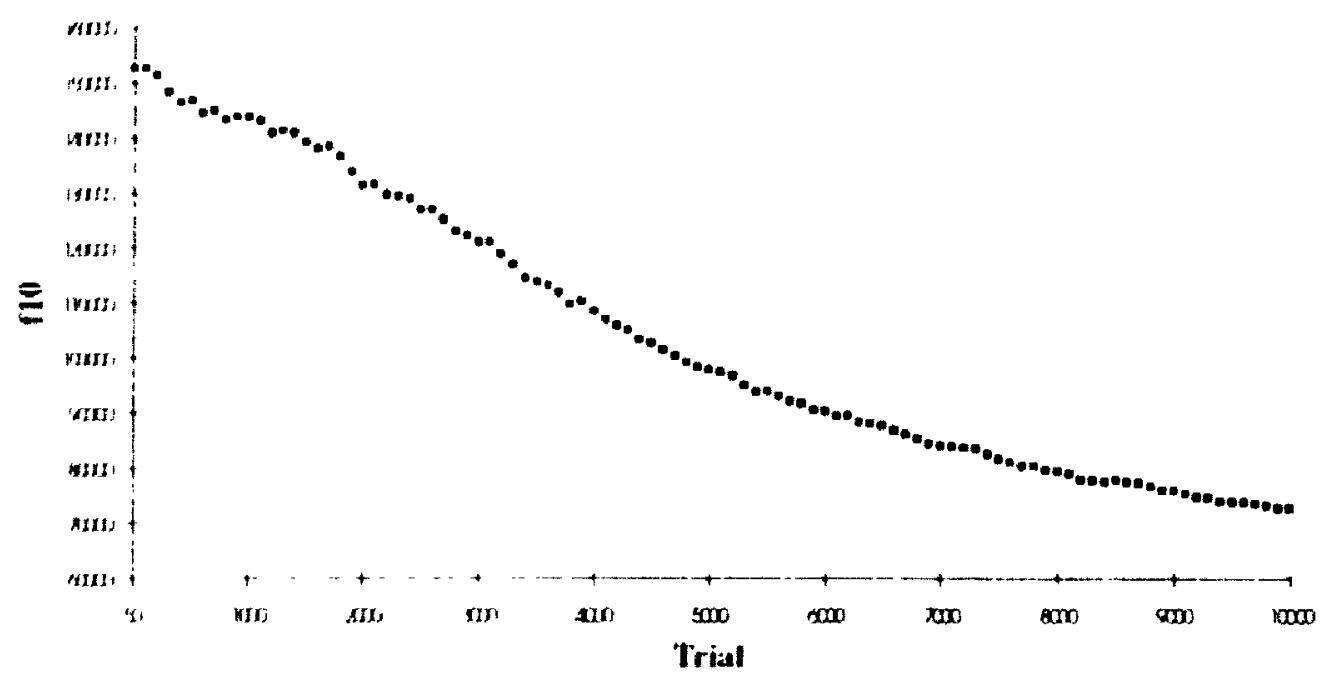

\section{Figure 6.3 fII) adaptive firness}

\section{Phot of rio vermas Trial for adaptive run}

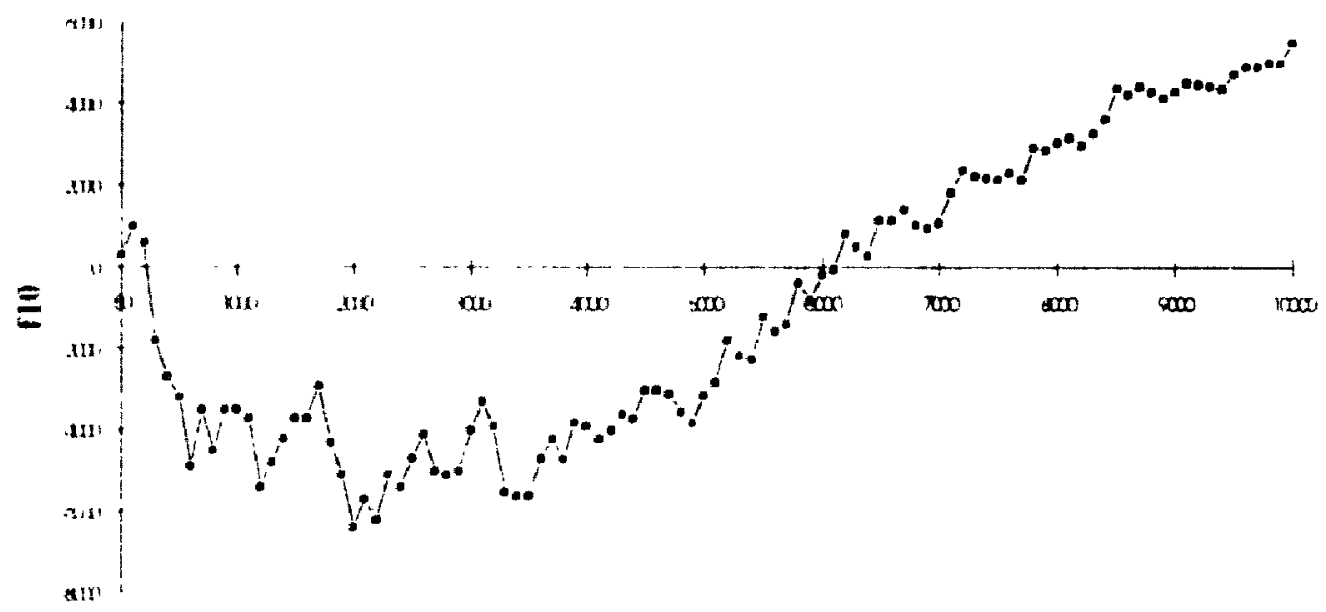

'Trial

Fïgure of f10 adaptive - mon-adaptive comparison 


\section{B.1.11 Low Autocorrelation Binary Sequences}

\section{Phot of fll 1 ersus Trial her mon-.tditptoce run}

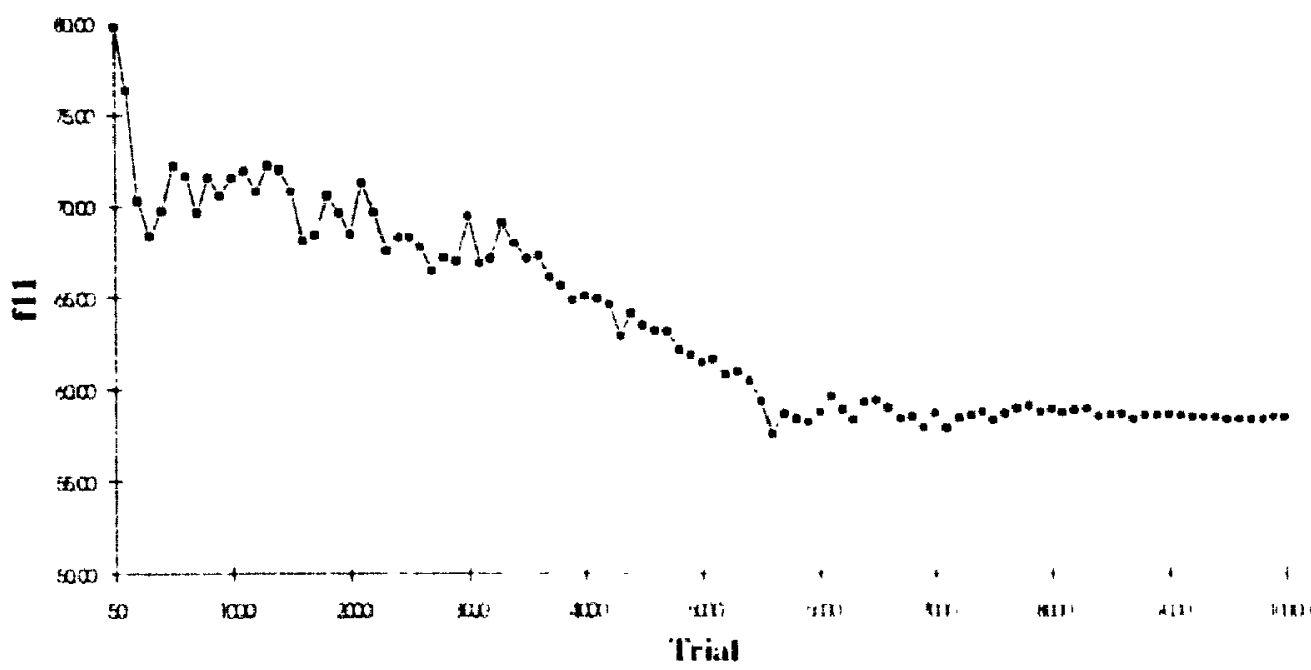

Figure 65 fll non-adaptive fitness

Mot of fll vervas Irial for adaptive ran

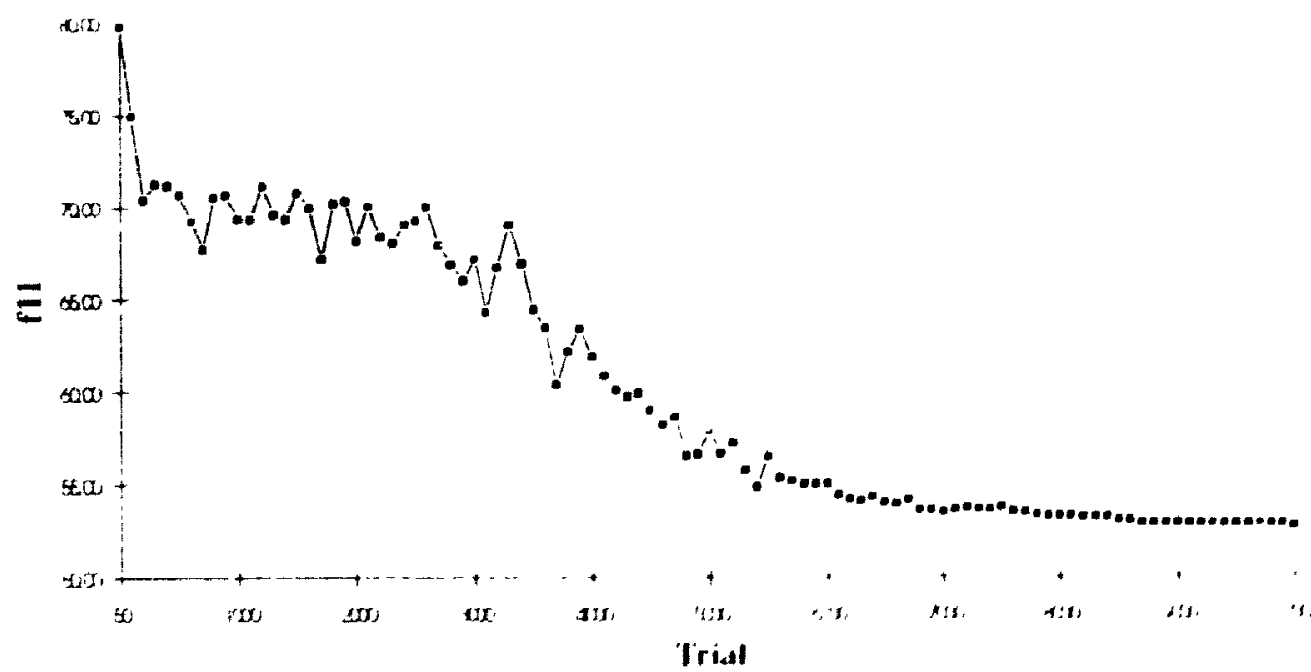

Figure 66 fll adaptive litness 

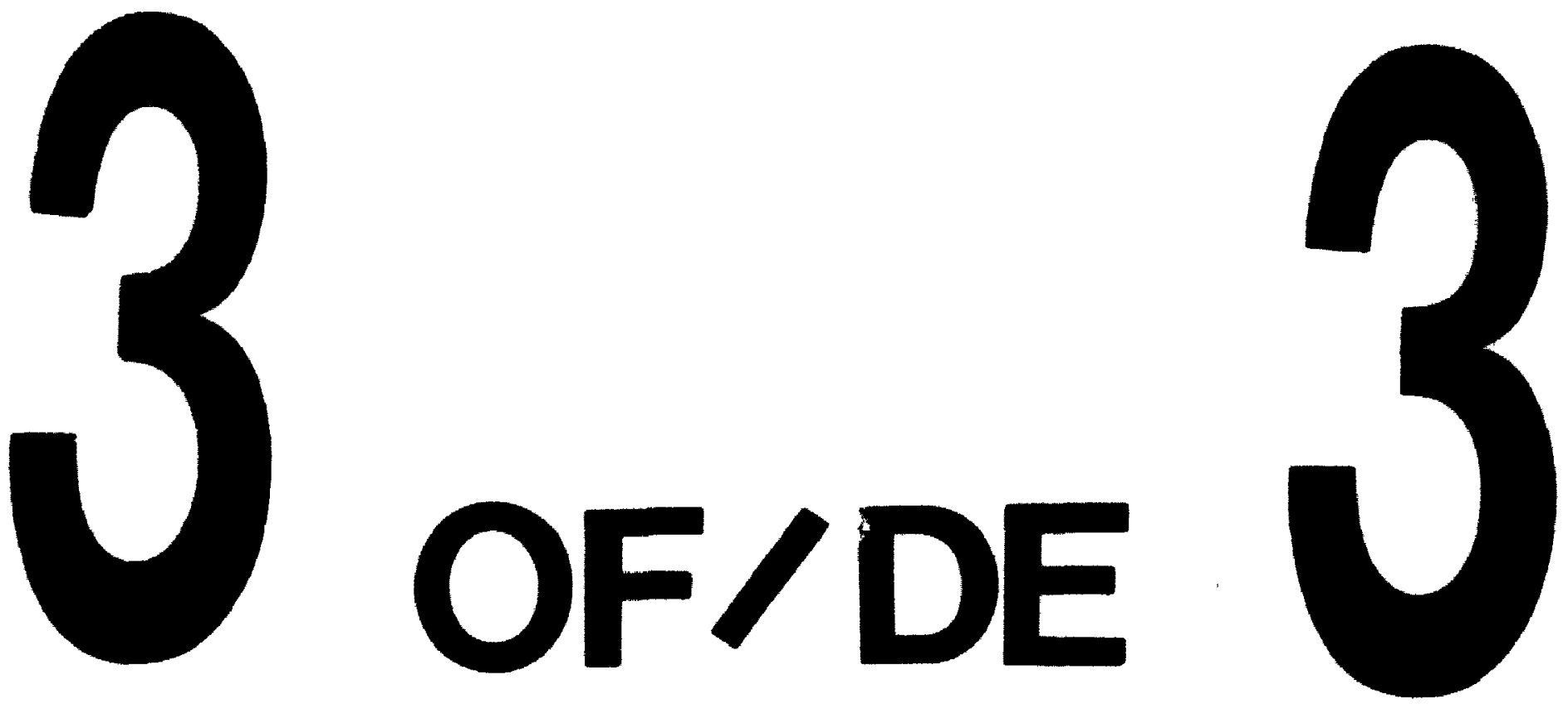

PM-1 3\%" $\times 4$ " PMOTOGAAPHIC MICROCOPY TARGET NBS 1010 a ANSI/ISO "2 EOUIVALENT

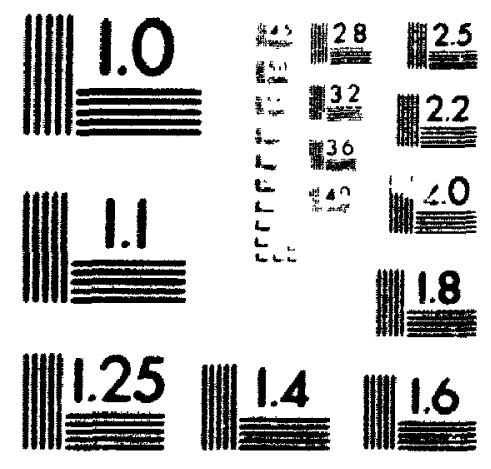


Plot of fll versus Trial differeme hetween adaptive and men-adaplic rums

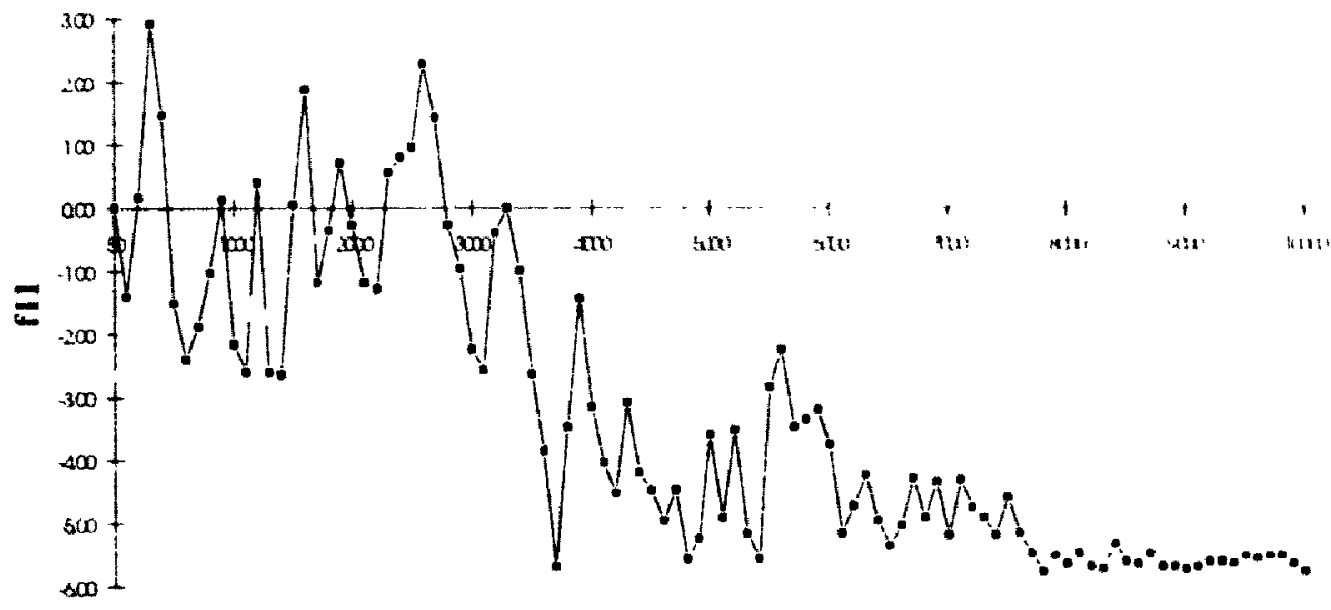

Trial

Figure 67 fll adaptive - non-adaptive comparisor: 


\section{B.1.12 Hamming distance to $\mathrm{O}^{\prime}$ (origin)}

Inot of fi2 versus Trial for mon-adaptive run

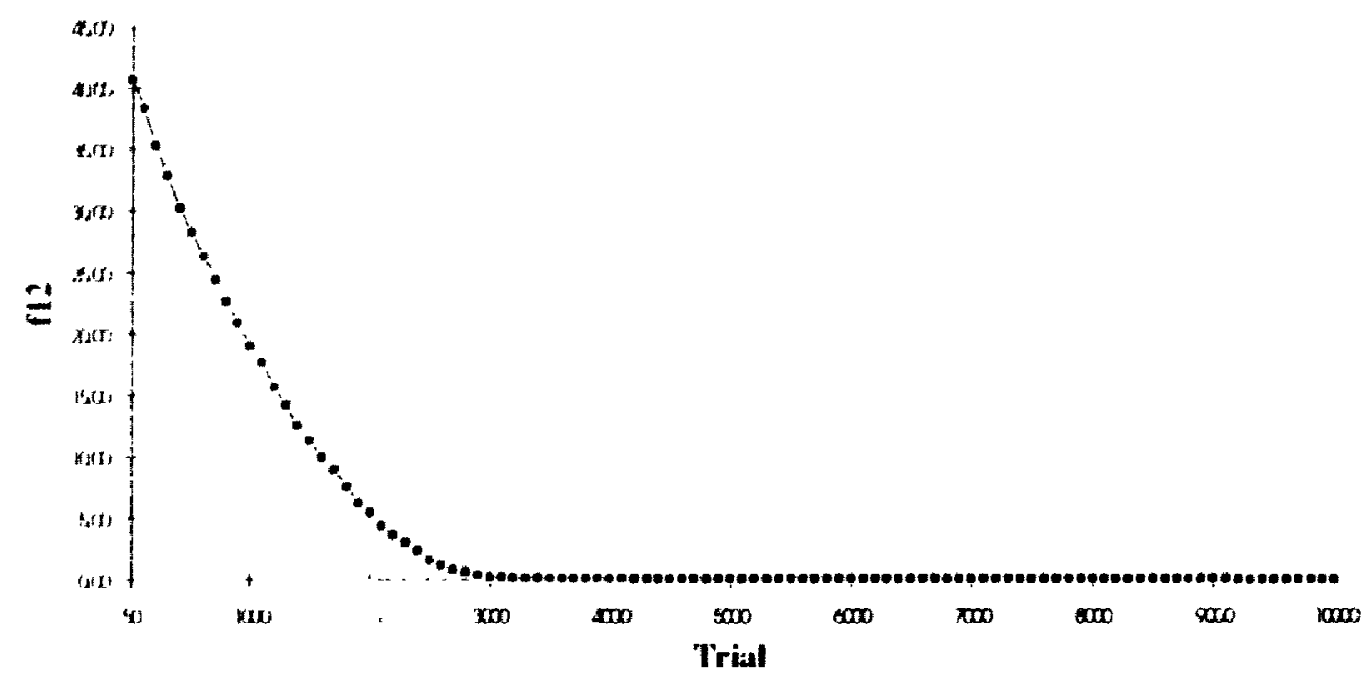

\section{Figure 6.x 112 non-adaptive fitness}

Mot of 72 versus Trial for adaptive run

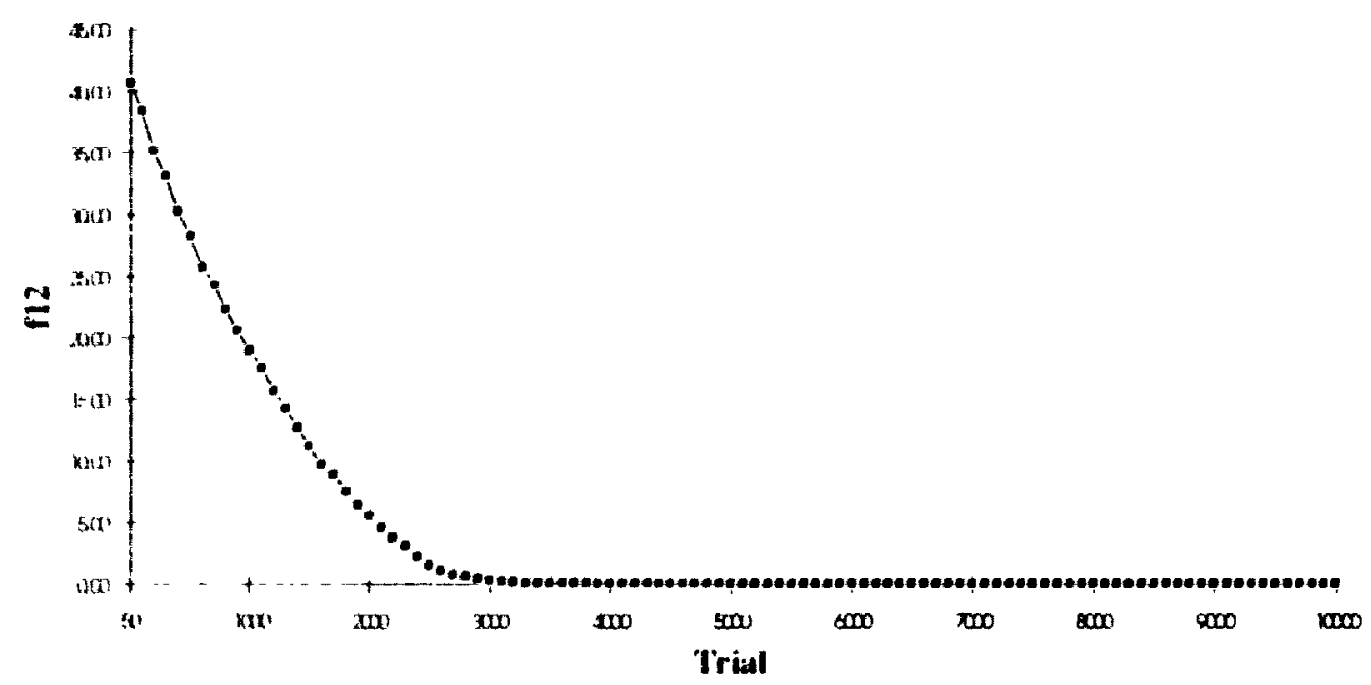


Phot of 72 versus Trial difference hetween adaptive and muln-adaptive tums

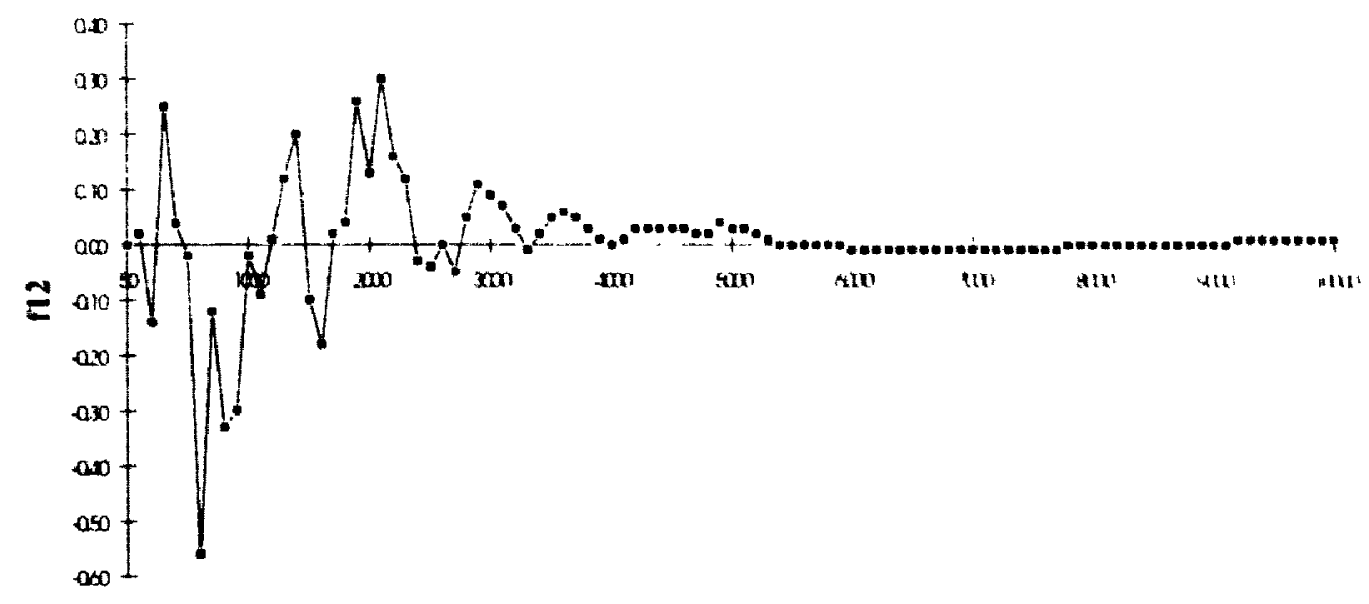

Trial

Figure 70 12 adaptive - non-adaptive comparison

\section{B.1.13 Weierstrass-Mandelbrot Fractal Function}

Plot of fl 3 versus Trial for nen-adaptive run

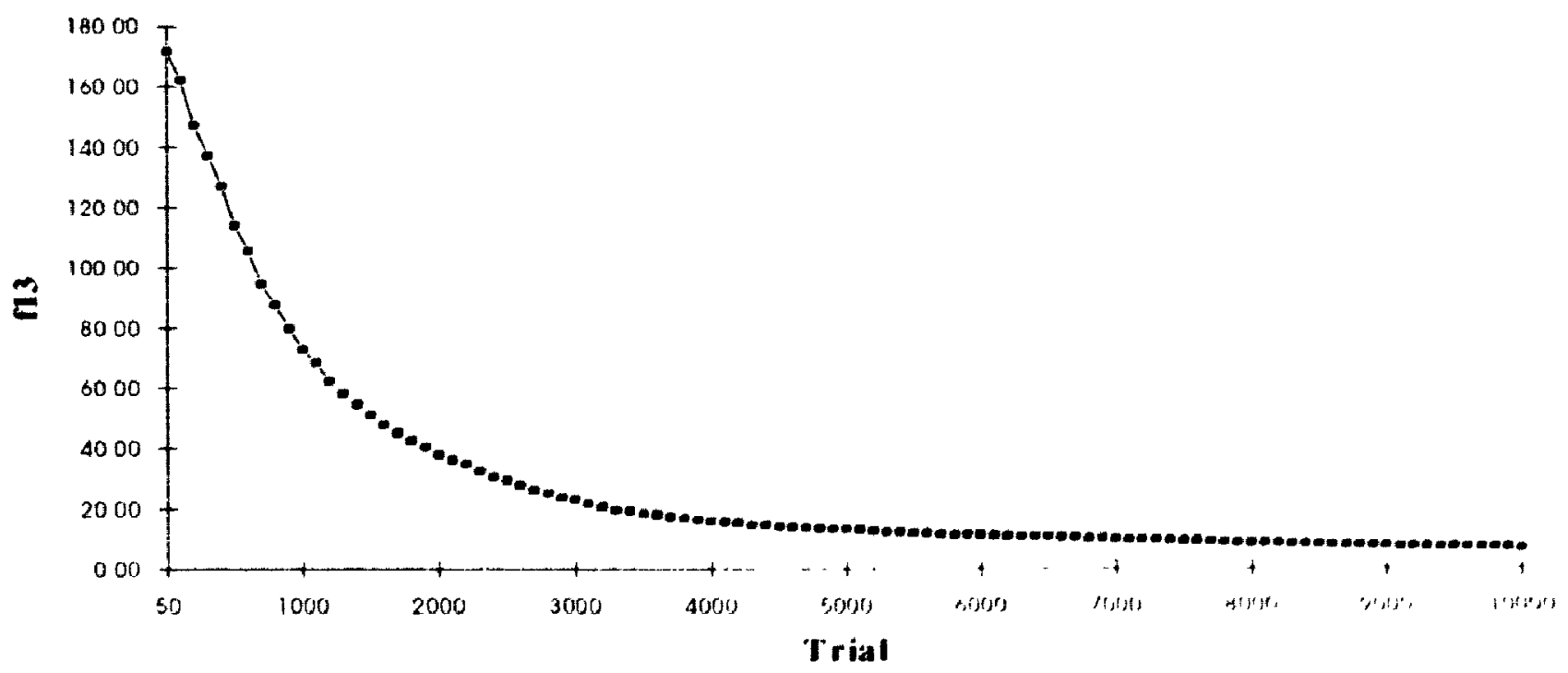

Figure 71 f13 nun-adaptive fitnes 
$\mathbf{s}$

Plol of f1.3 versus Trial for adaptive run

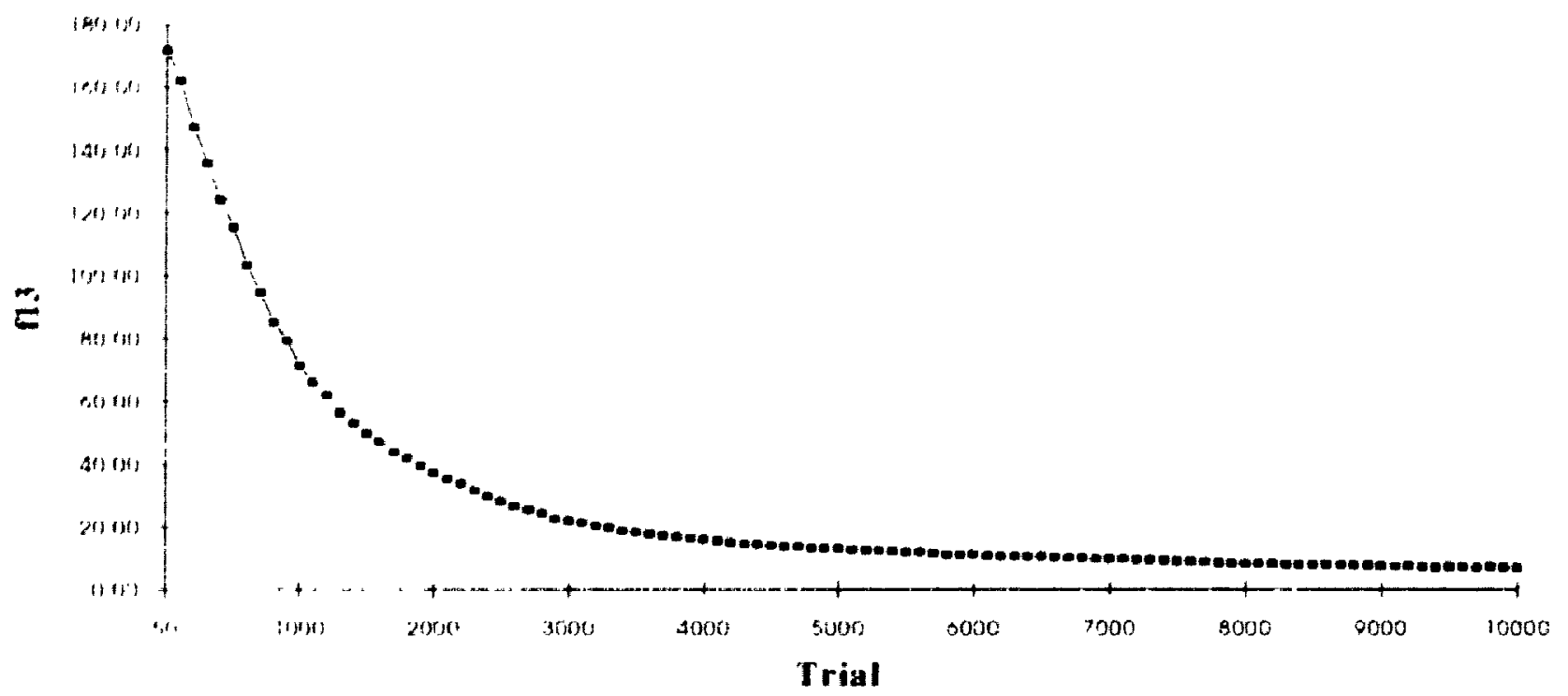

Figure 72 f1.3 adaptive fitness

Plot of 13 versus $T$ rial difference between adaptive and non-adaptive runs

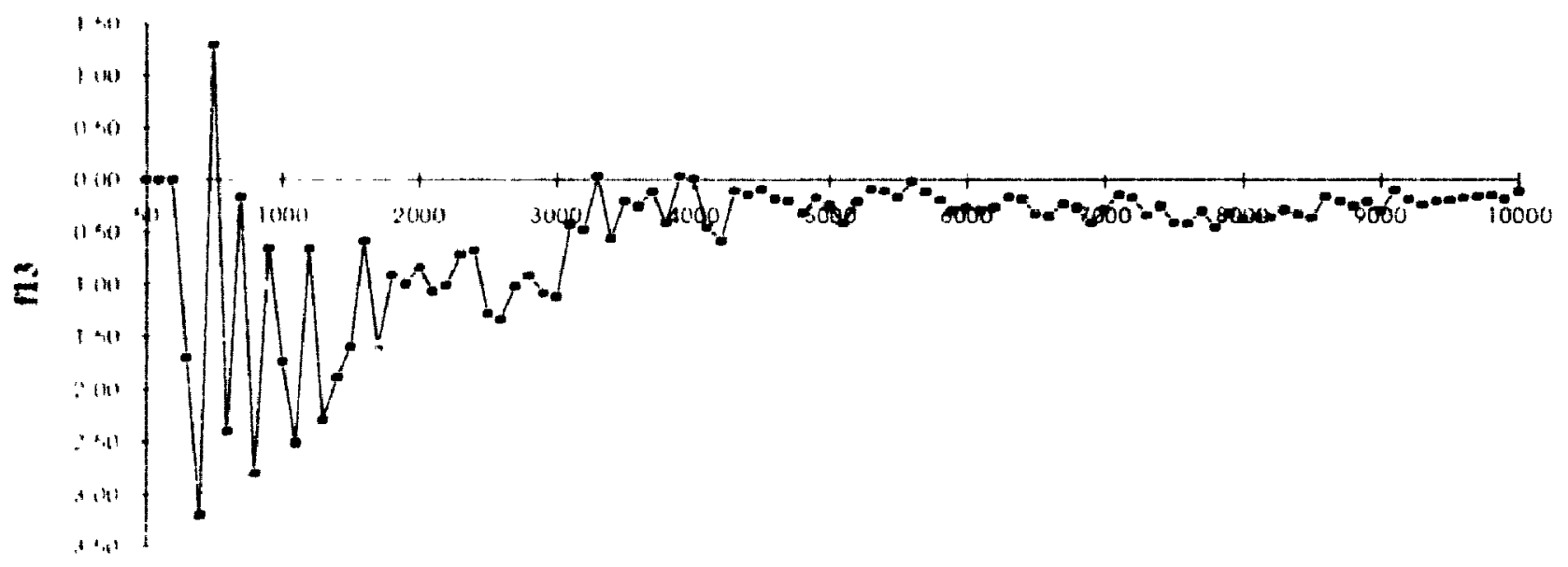

Trial

Figure 7.311 .3 adaptive - non-adaptiv - comparison 


\section{B.1.14 Fully Deceptive Function}

\section{Mot of fit versus Trial fior nom-adaptice run}

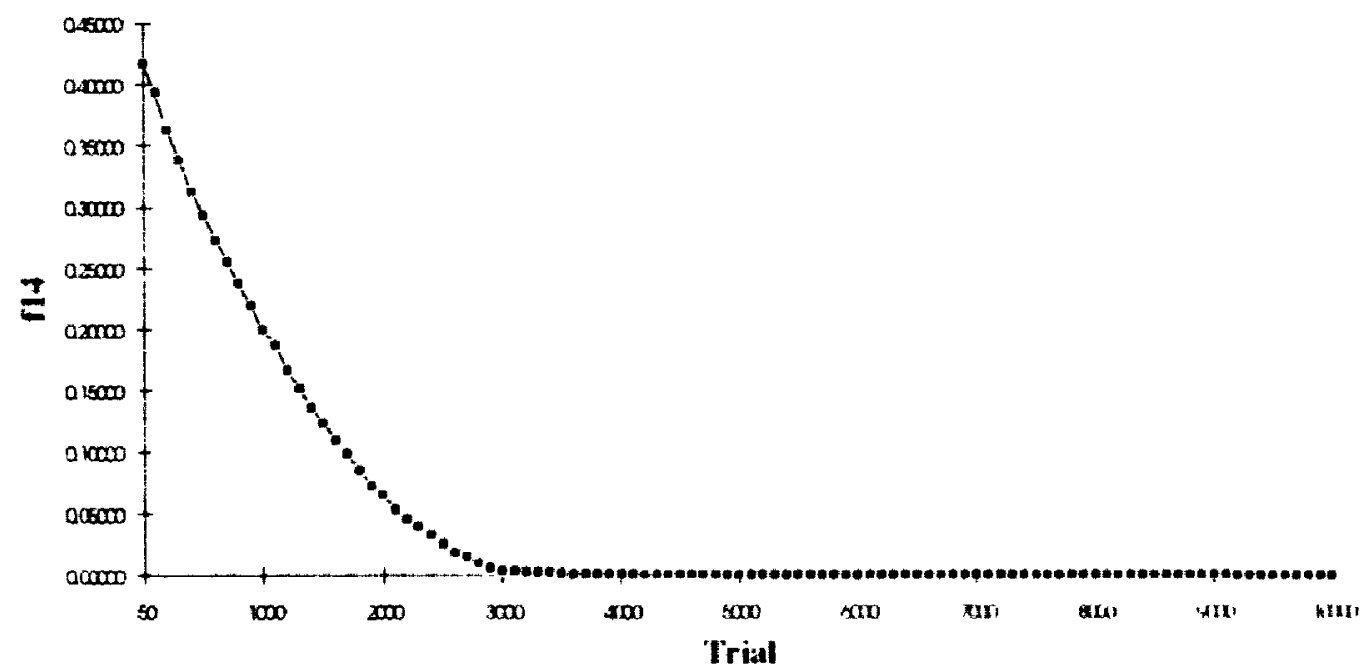

\section{Figure 74 f14 non-adaptive fitness}

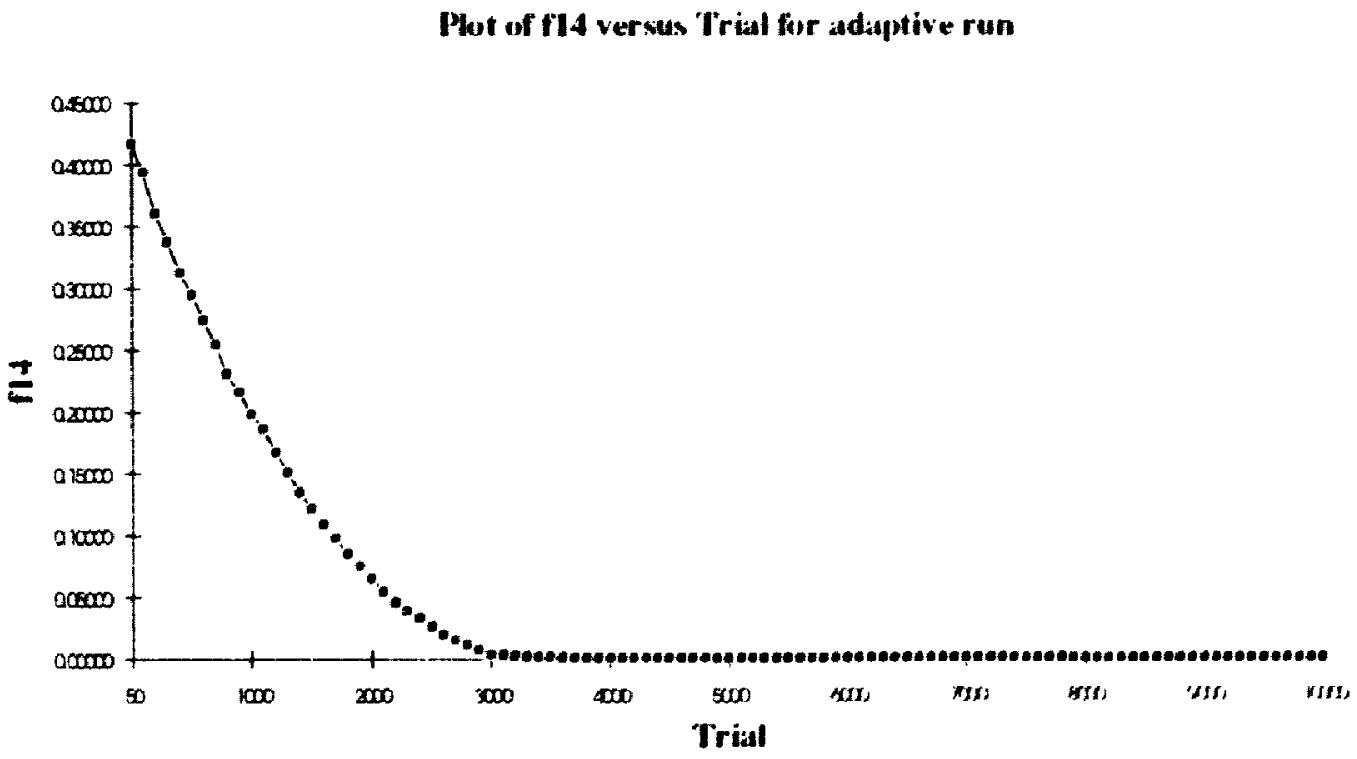

Figure $75 \mathrm{f14}$ adaptive fitness 
Plot of fld versus Trial difference between adaptive and non-adaptive runs

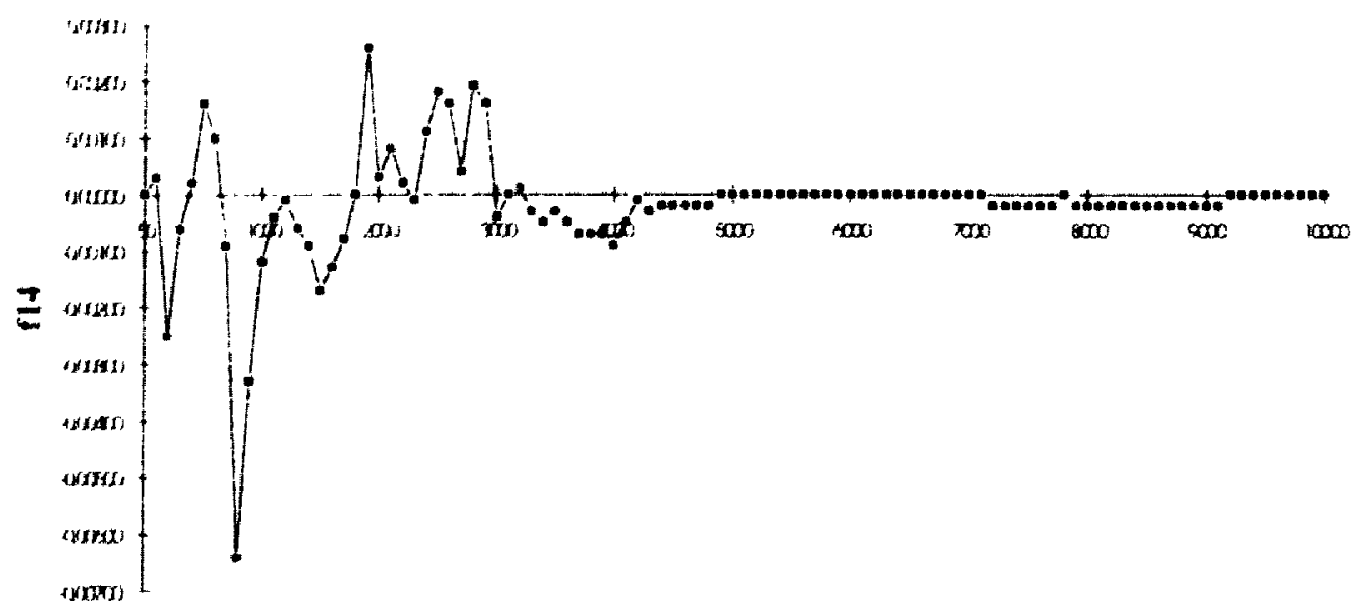

Trial

Figure 76 I14 adaptive - non-adaptive comparison

\section{B.1.15 Weighted Sphere Model}

Phot of fis versus Trial for non-adaptive run

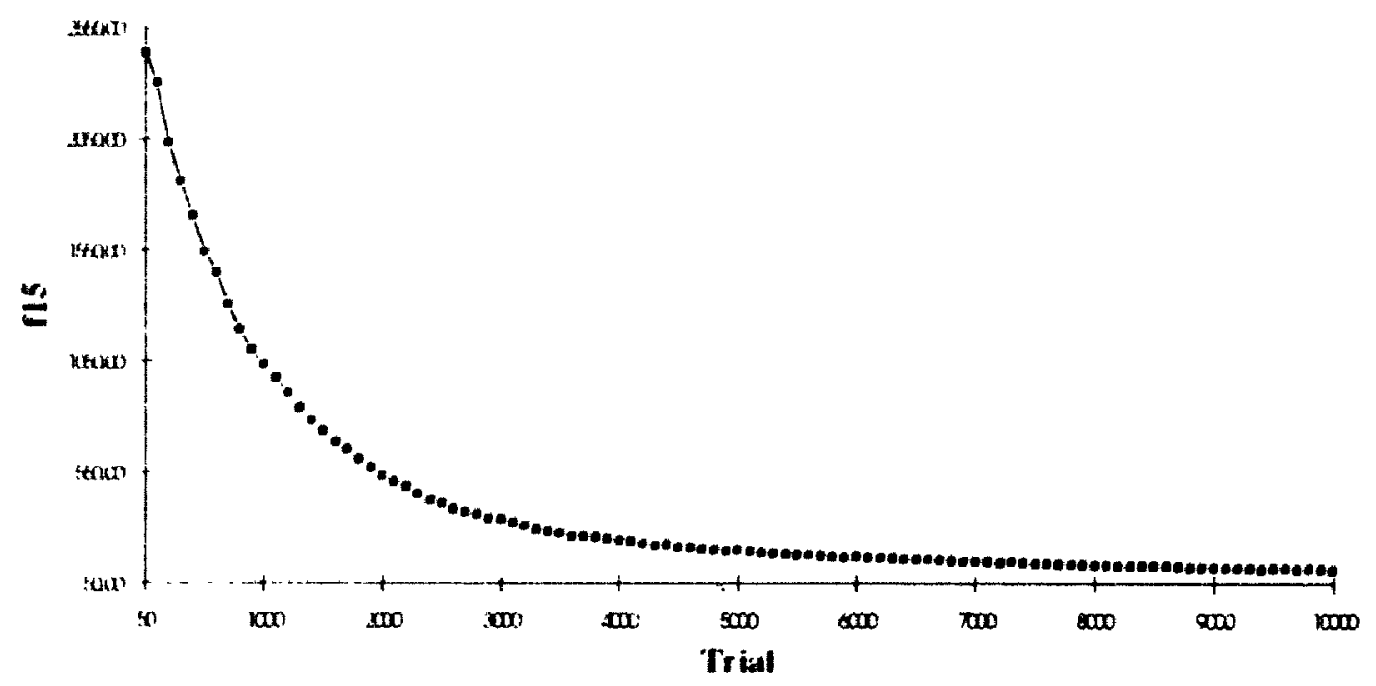

Figure 77 f15 non-adaptive fitness 


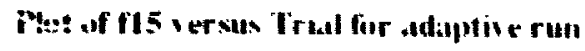

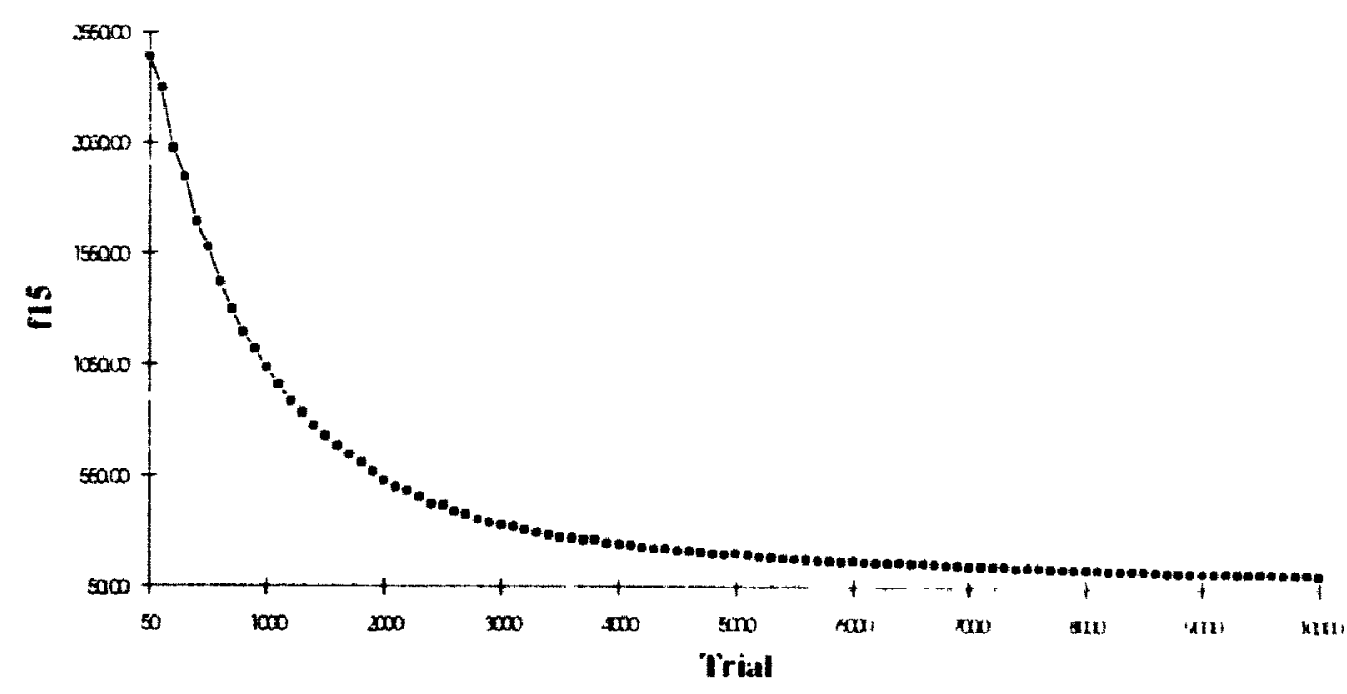

\section{Figure 78 f15 adaptive fitness}

Phot of f15 versus Trial difference letween adaptive and notn-idaptive runs

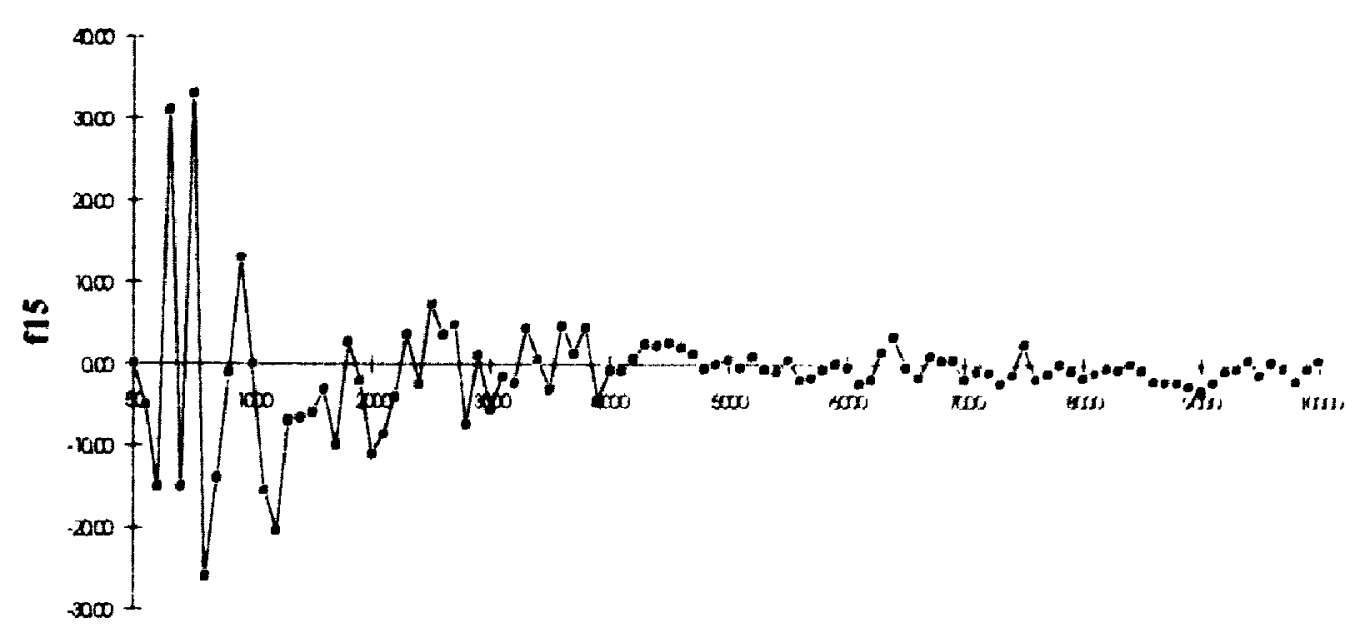

Trial

Figure 79 f15 adaptive - non-adaptive comparison 


\section{B.1.16 Fletcher and Powell (fixed A,B matrices)}

Plot of fis verwas Trial for mon-adaptive run

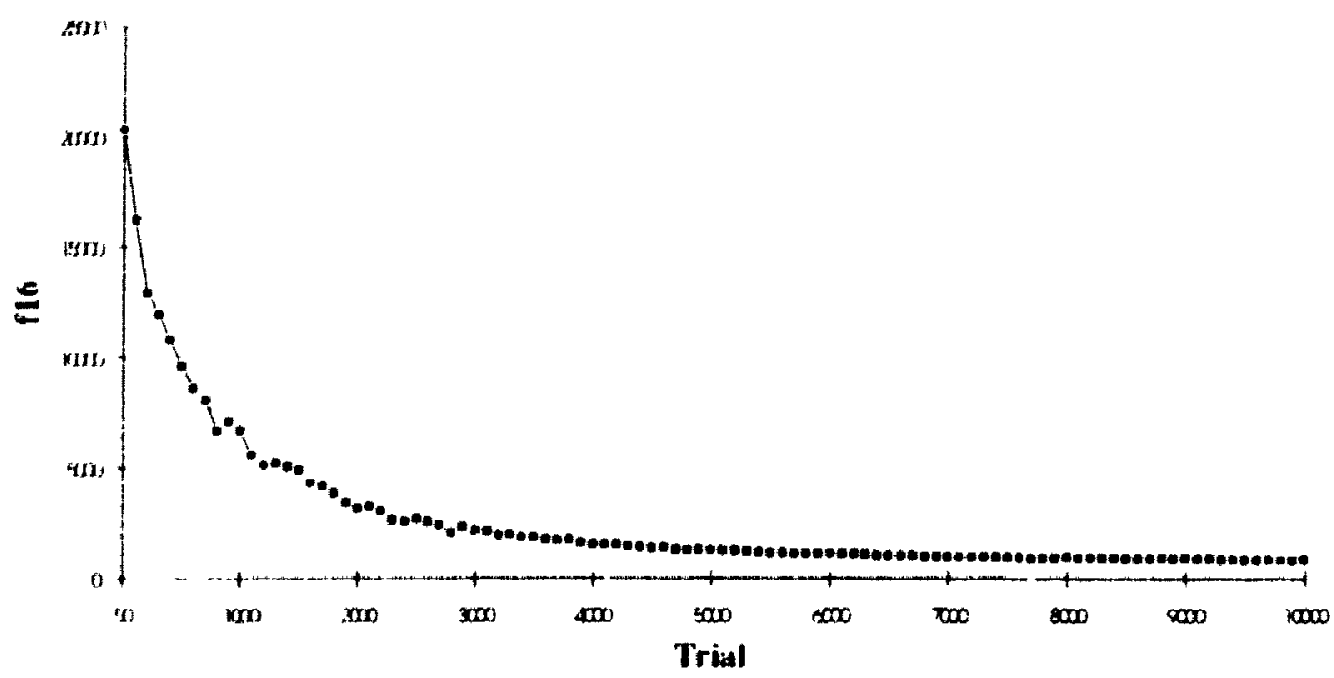

\section{Figure 80116 non-adaptive fitness}

Put of f16 versus 'Trial for adaptive runi

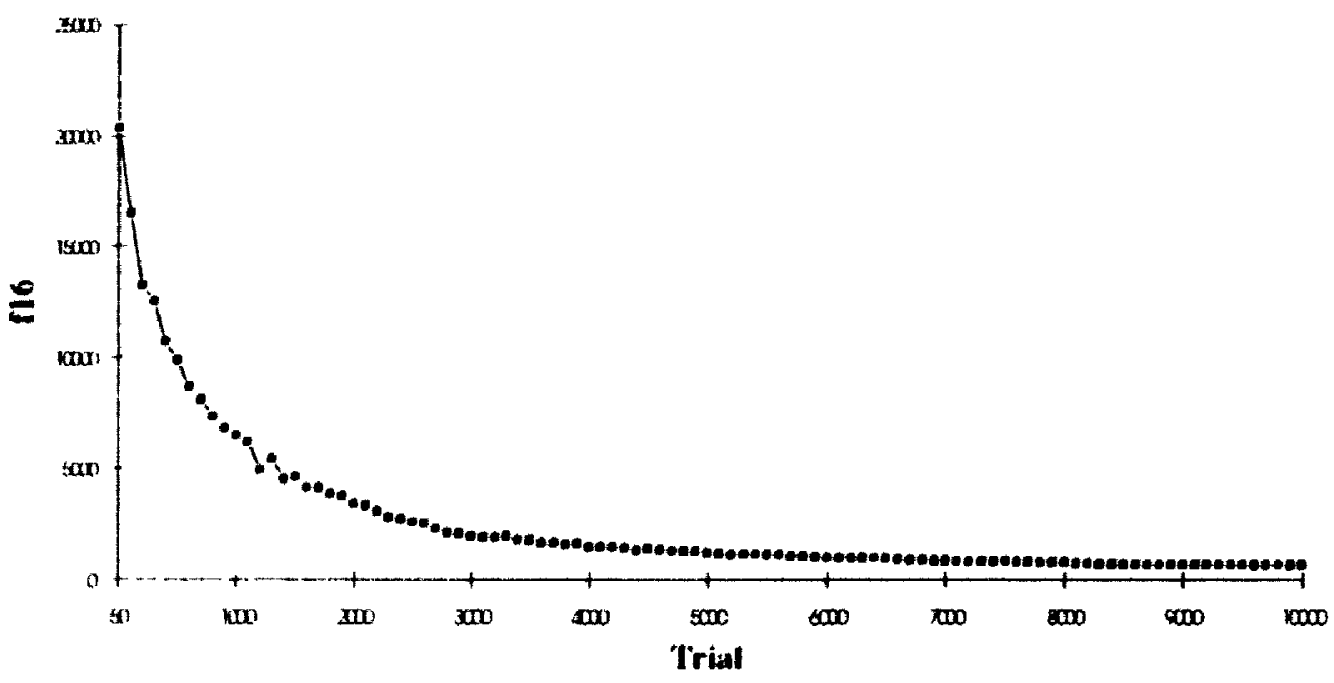


Phot of fis versus Trial difference between adaptive and nutradaptive runs

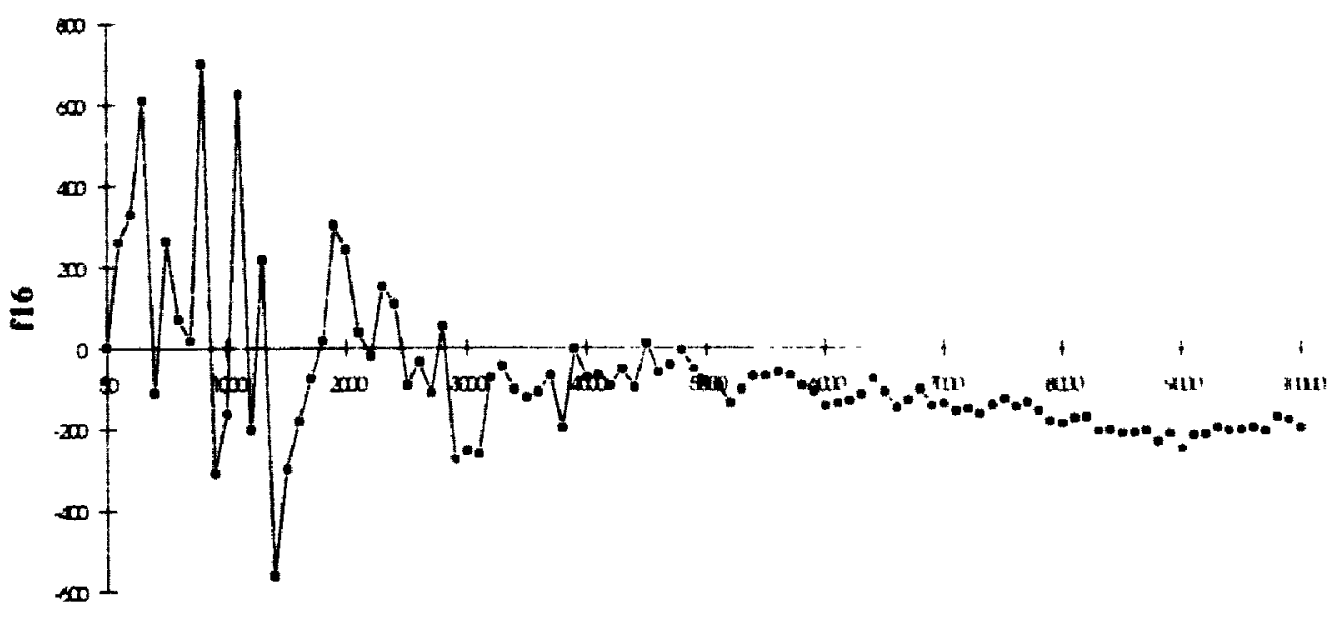

Trial

Figure 82116 adaptive - non-adaptive comparison

\section{B.1.17 Flatcher and Powell (with random A,B matrices)}

Phot of 197 verwas Trial for mon-adaptive run

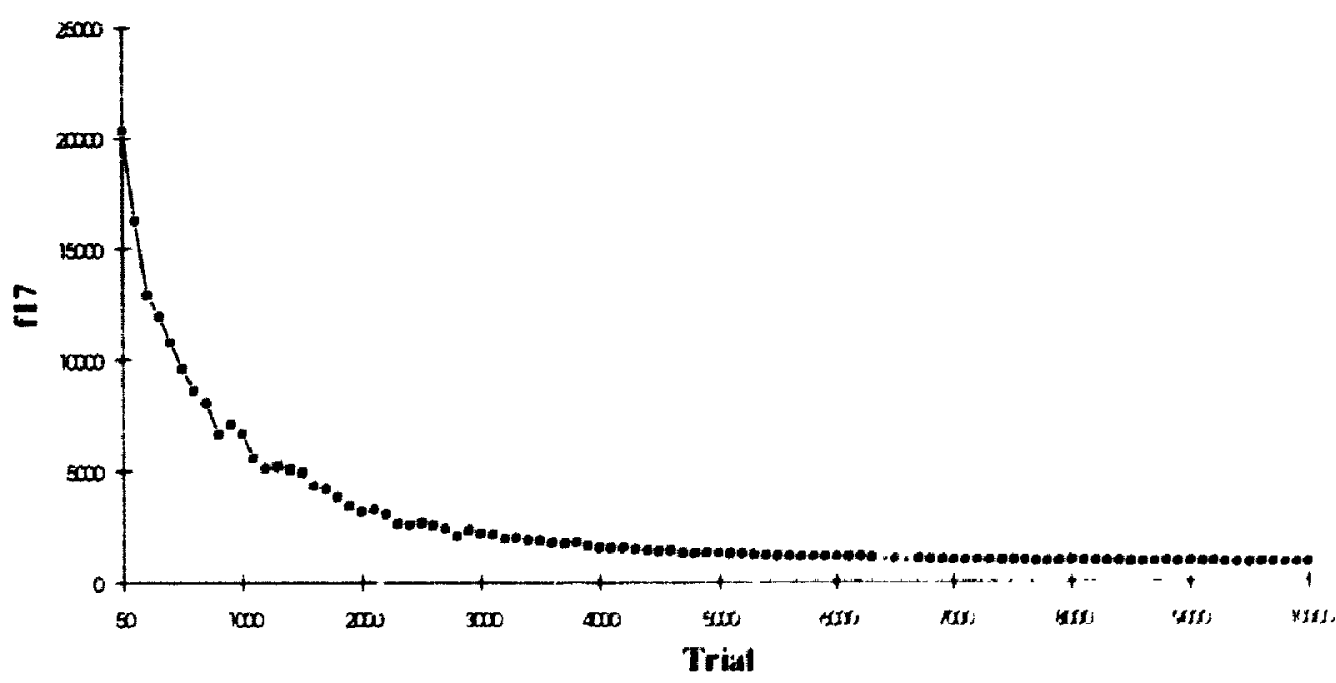

Figure $83 \mathbf{8 1 7}$ non-adaptive fitnes 
S

Hot of fi7 versus Trial for adaptive run

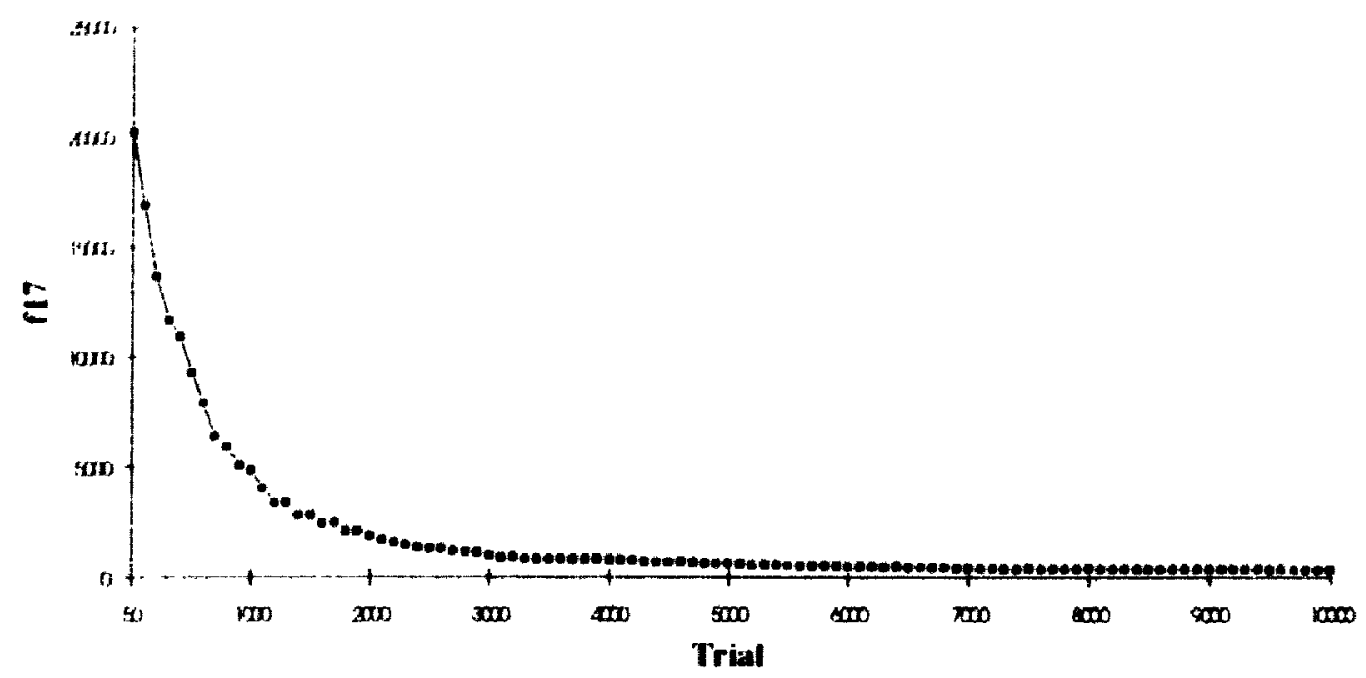

Figure $\$ 4 \mathbf{~} 17$ adaptive fitness

Pht of fI7 verwas Trial difference between adaptive and non-adaptive runs

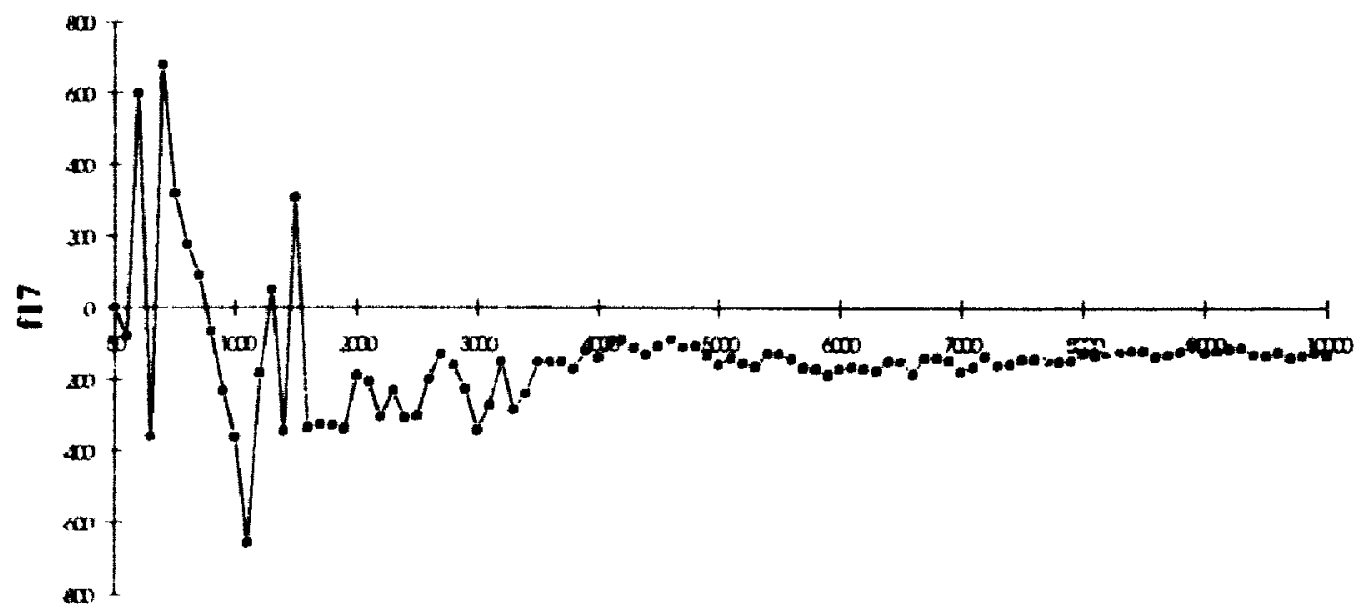

Trial

Figure $85 \quad 17$ adaptive - non-adaptive comparison 


\section{B.1.18 Shekel-5}

Mot of flX versus Trial fior mon-indaptive run

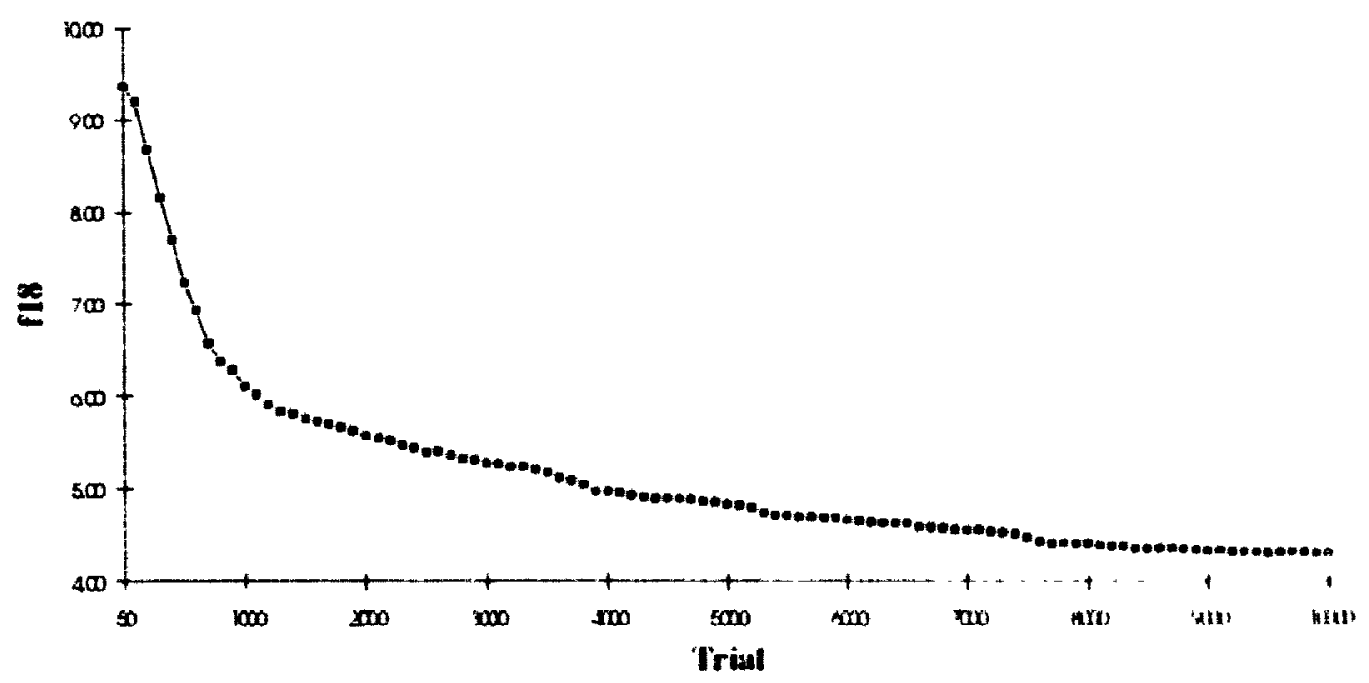

Figure 8678 non-adaptive fitness

Phet of fis versus Trial for adaptive run

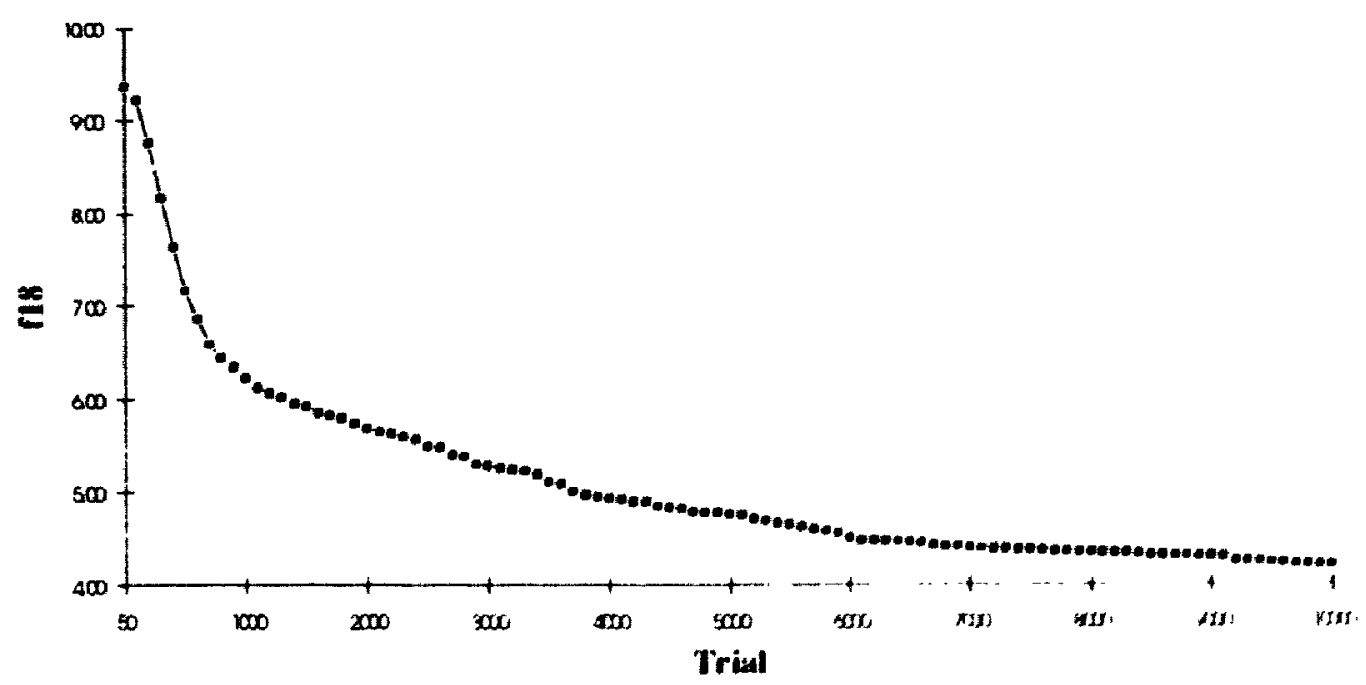

Figure 87 II8 adaptive fitness 
Phit of tis verus 'Trial difference between adaptive and non-adaptive runs

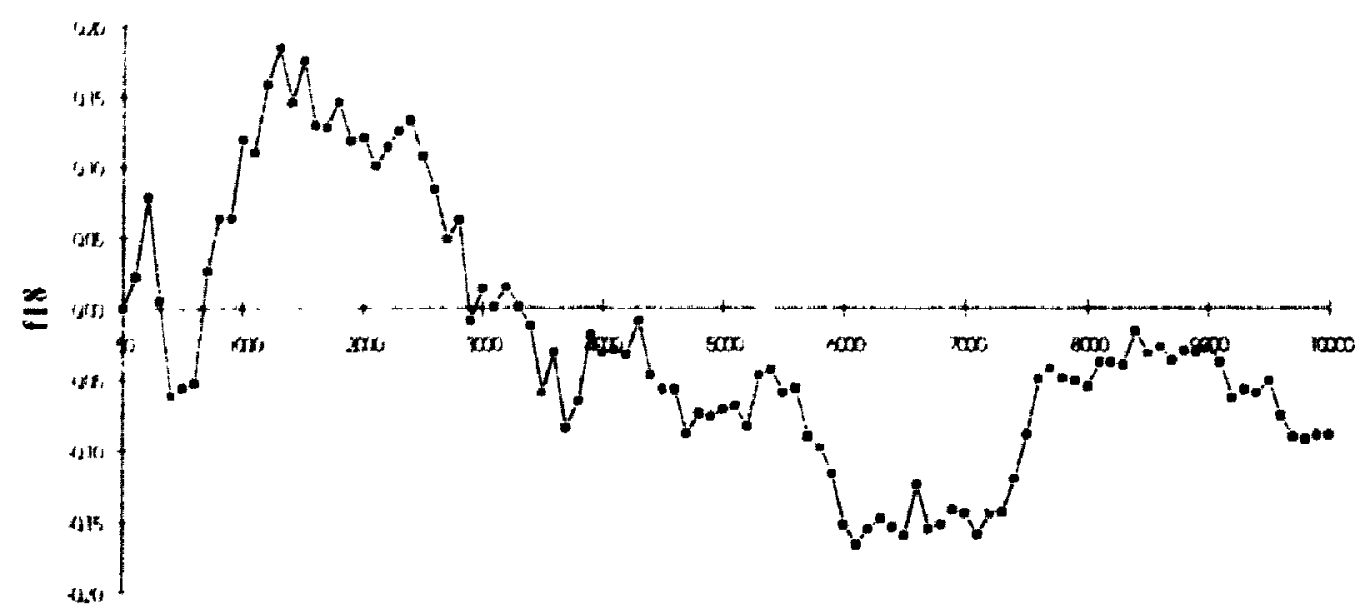

Trial

Figure 88118 adaptive - non-adaptive comparison

\section{B.1.19 Shekel-7}

\section{Phot of f19 verwus Trial for mon-uduptive run}

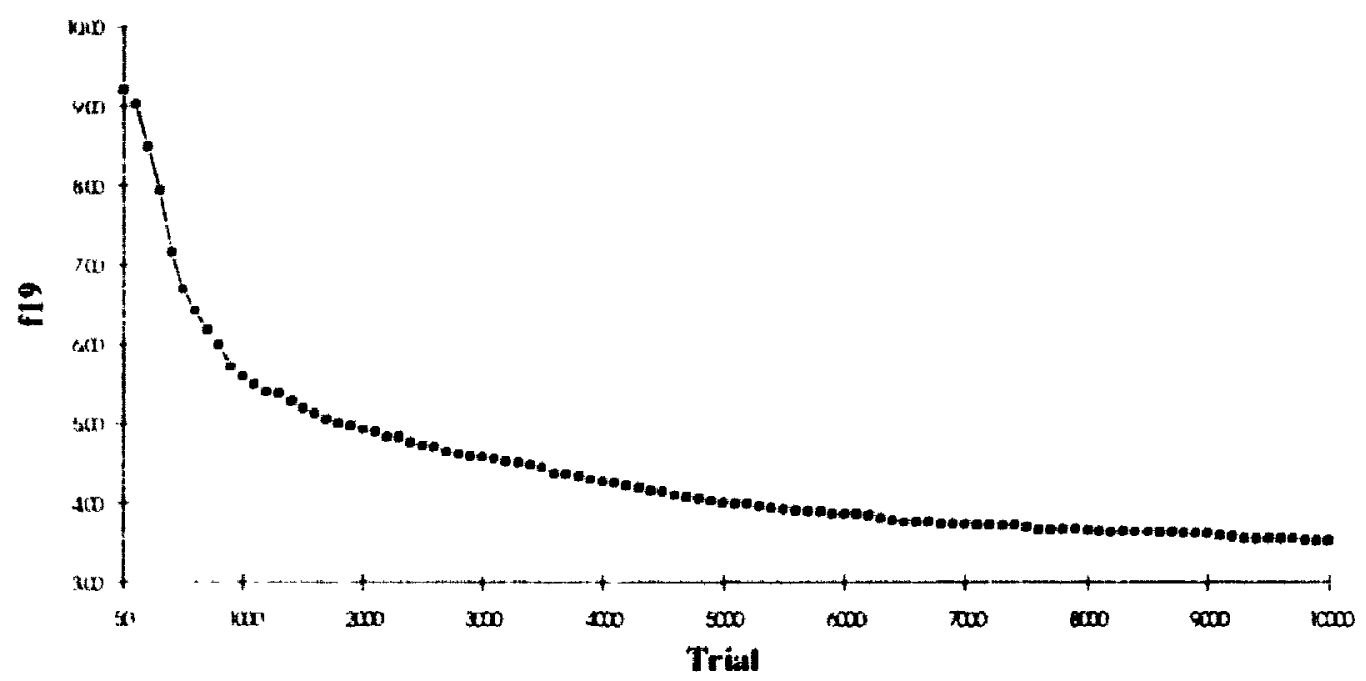

Figure 89719 mom-adaptive fitness 
Page 192

Appendix B: Results

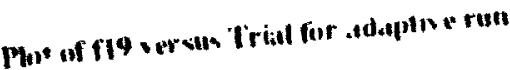

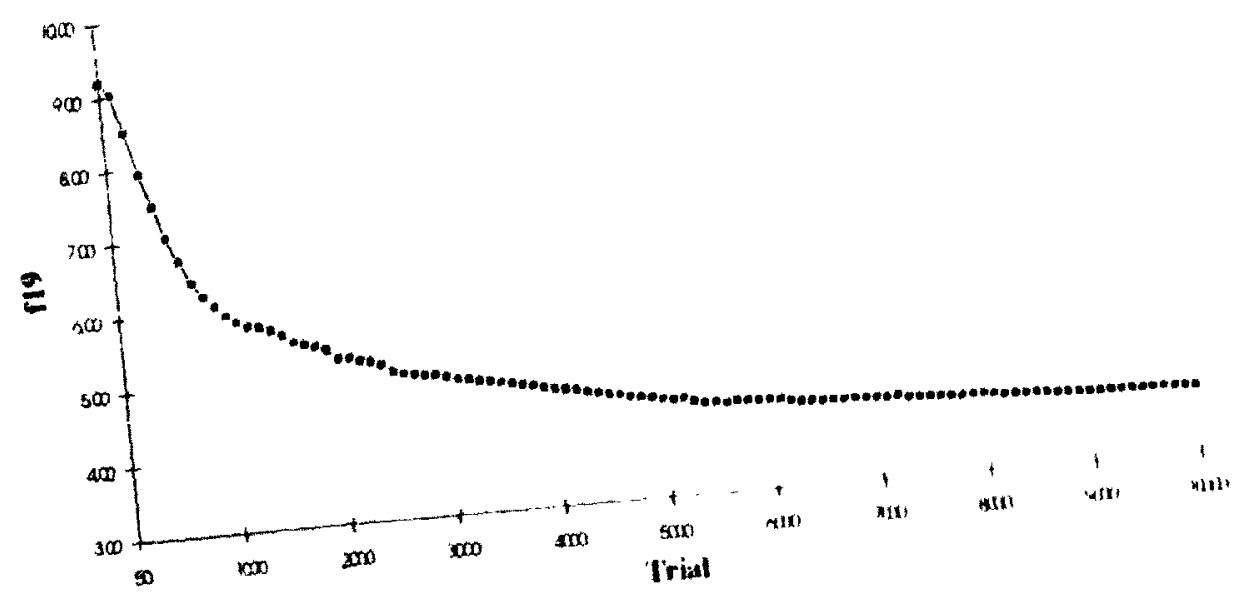

Figure $\% 019$ adaptive fitness

Phat of fl versus: Trial difference between adaptive and imon-adaptive rum

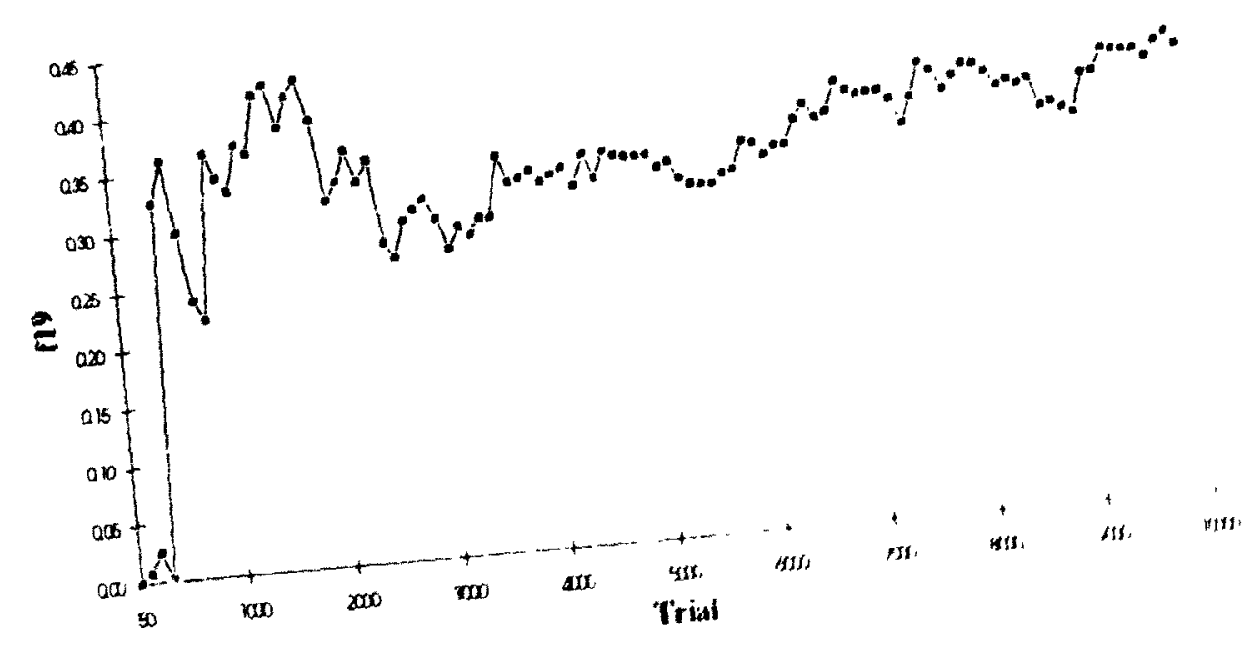

Figure 91 19 adaptive - nun-adaptive comparison 
Appendix H: Results

\section{B.1.20 Shekel-10}

Put of 120 versus Trial fur mon-adaptive run

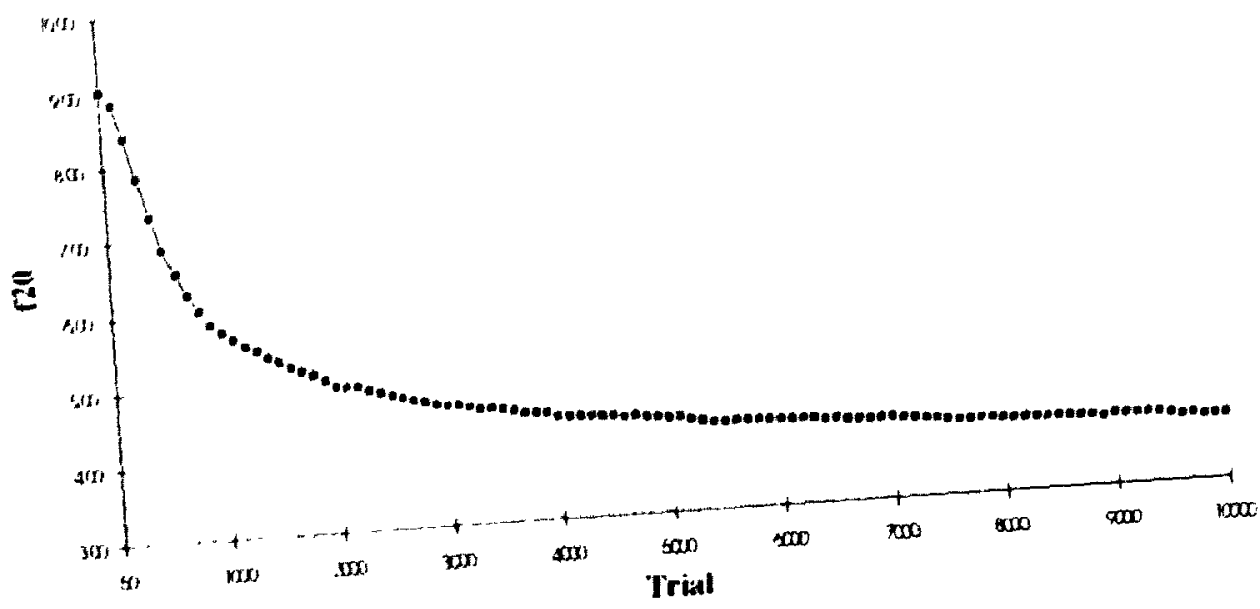

Figure 92 20 mon-adaptive fitness

Phot of Ro versus Trial for adaptive run

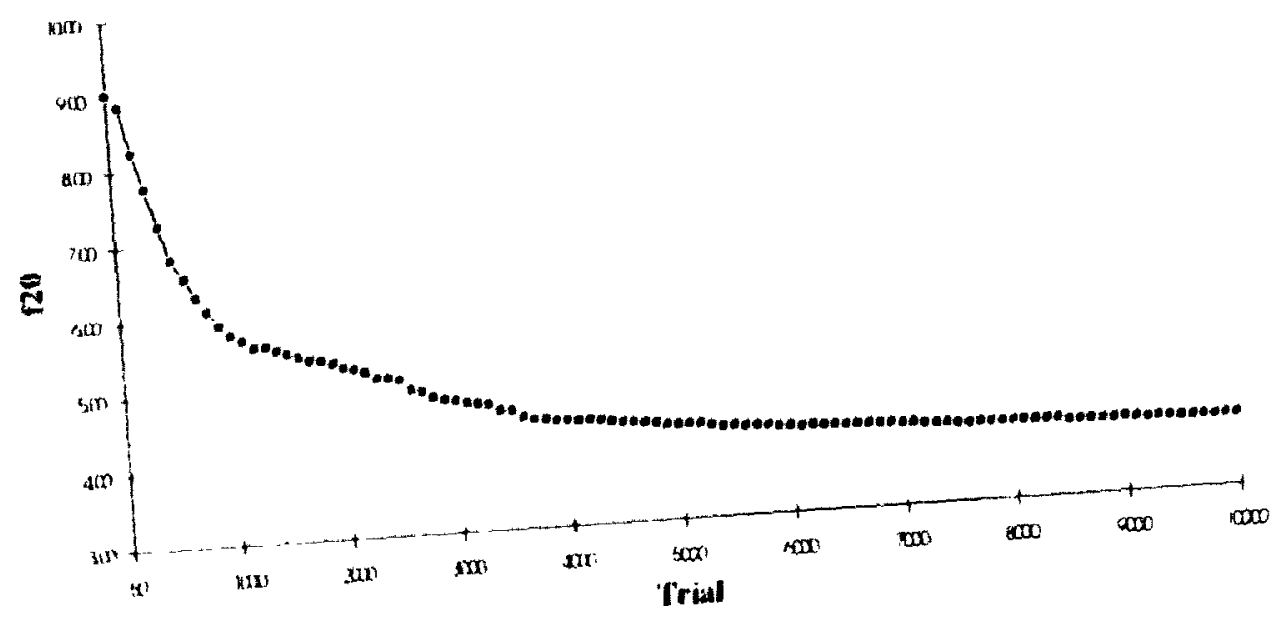

Figure 93 201 adaptive fitness 


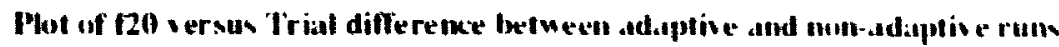

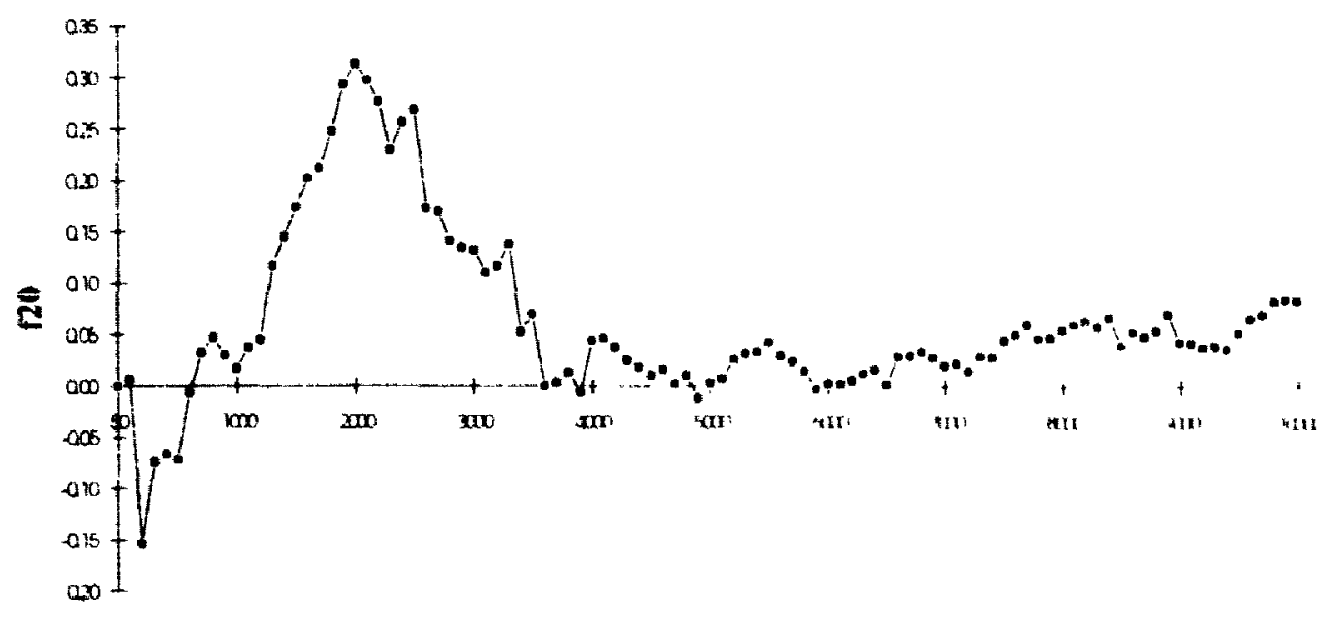

Trial

Figure 94 20 adaptive - mon-adaptive comparison

\section{B.1.21 Griewank-2}

Pot of 21 versus 'Trial for mon-iadaptive run

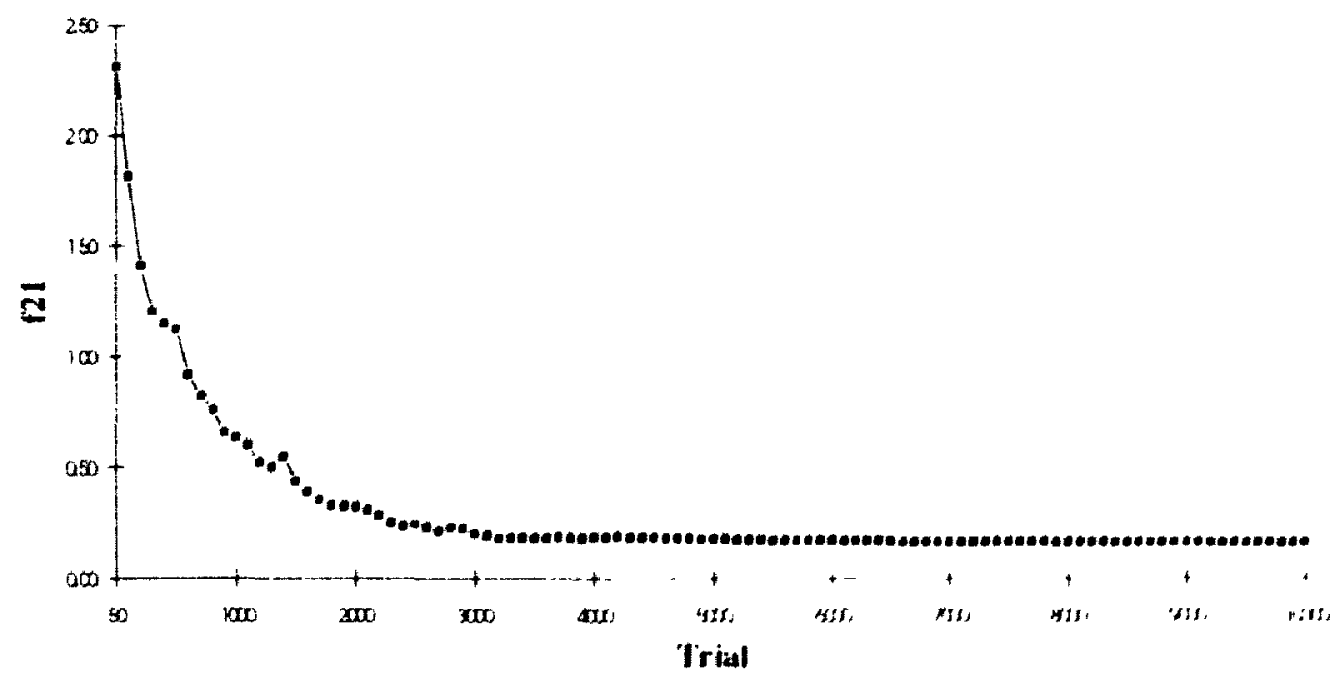

Figure 95 R1 non-adaptive fitness 
Met of $R$ I versus Trial for adaptive run

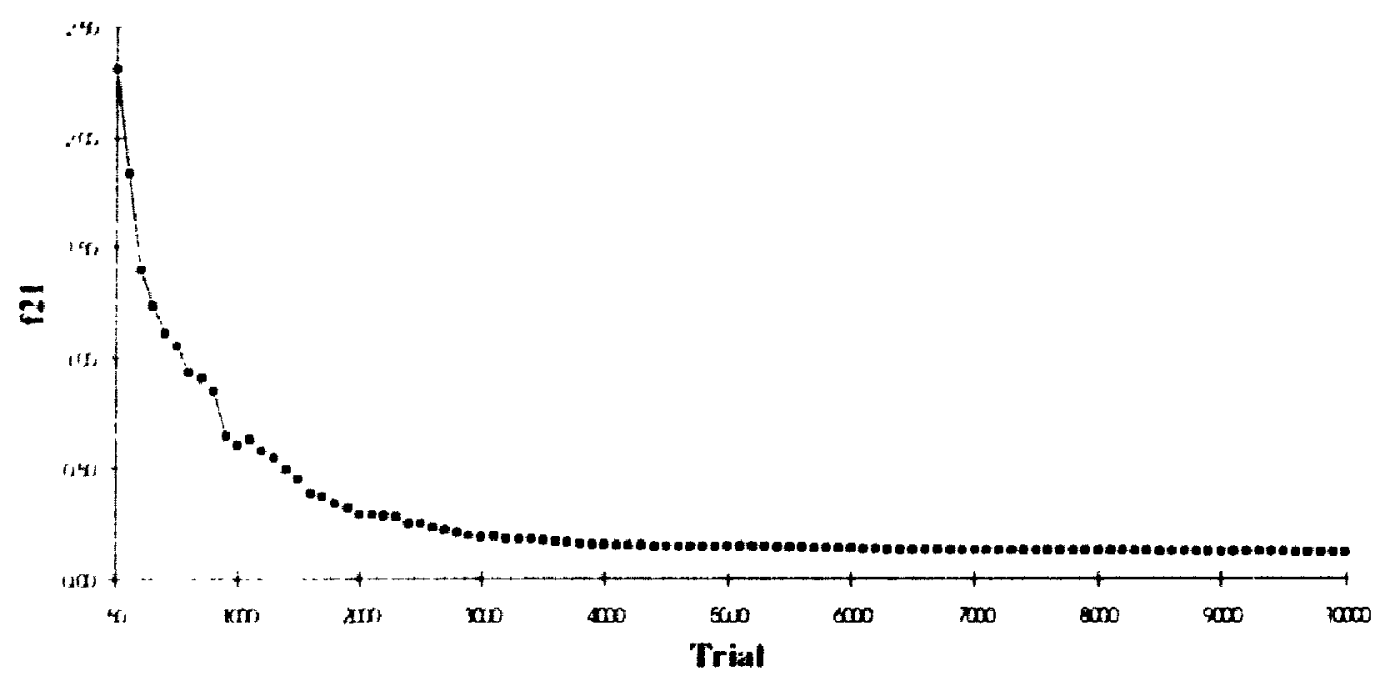

\section{Figure 96 21 adaptive fitness}

Hot of 21 versus 'Trial difference between adaptive and non-adaptive runs

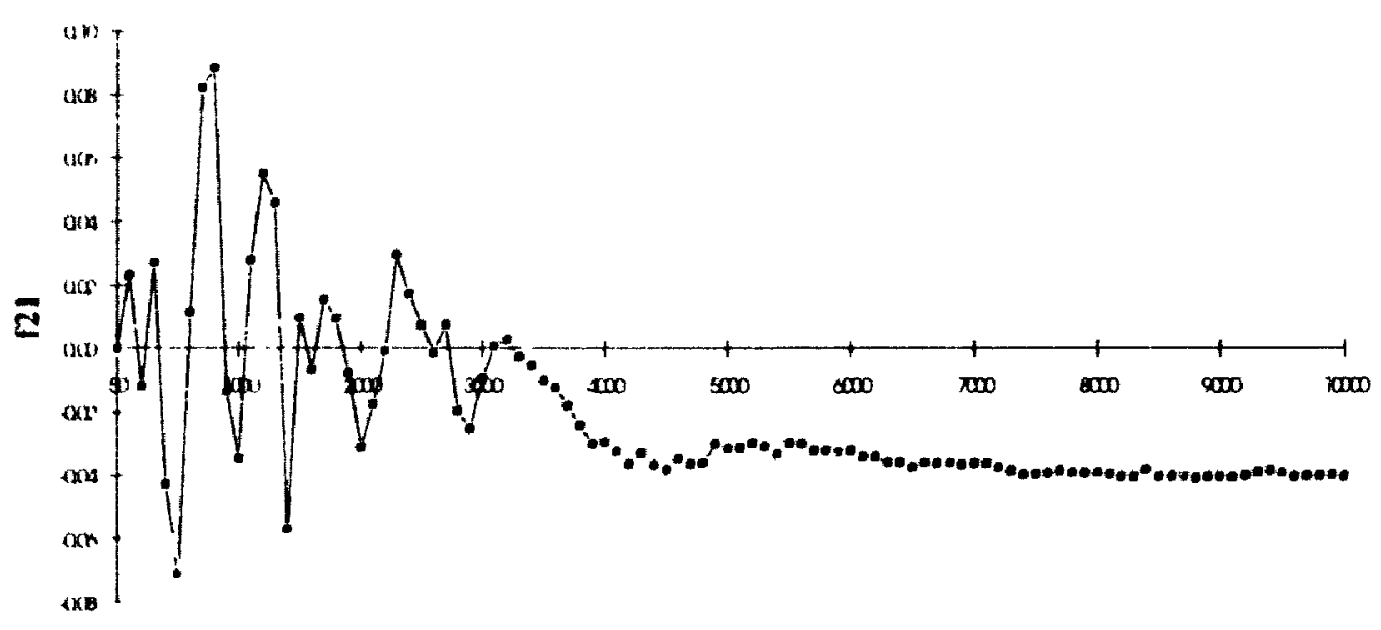

Triul

Figure 9721 adaptive - mon-adaptive comparison 
Appendix R: Results

Page 196

B.1.22 Griewank-10

Plot of fo versus Trial for mon-addiptise run

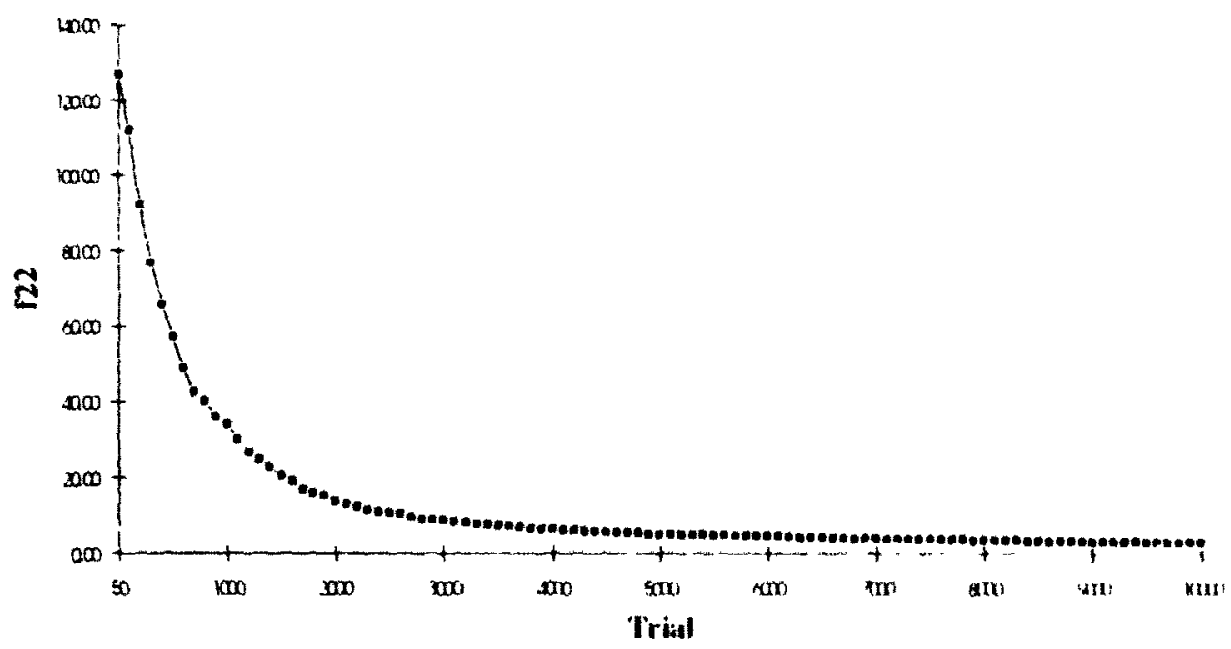

Figure 9822 non-adaptive fitness

Phot of 22 versus Trial for adaptive run

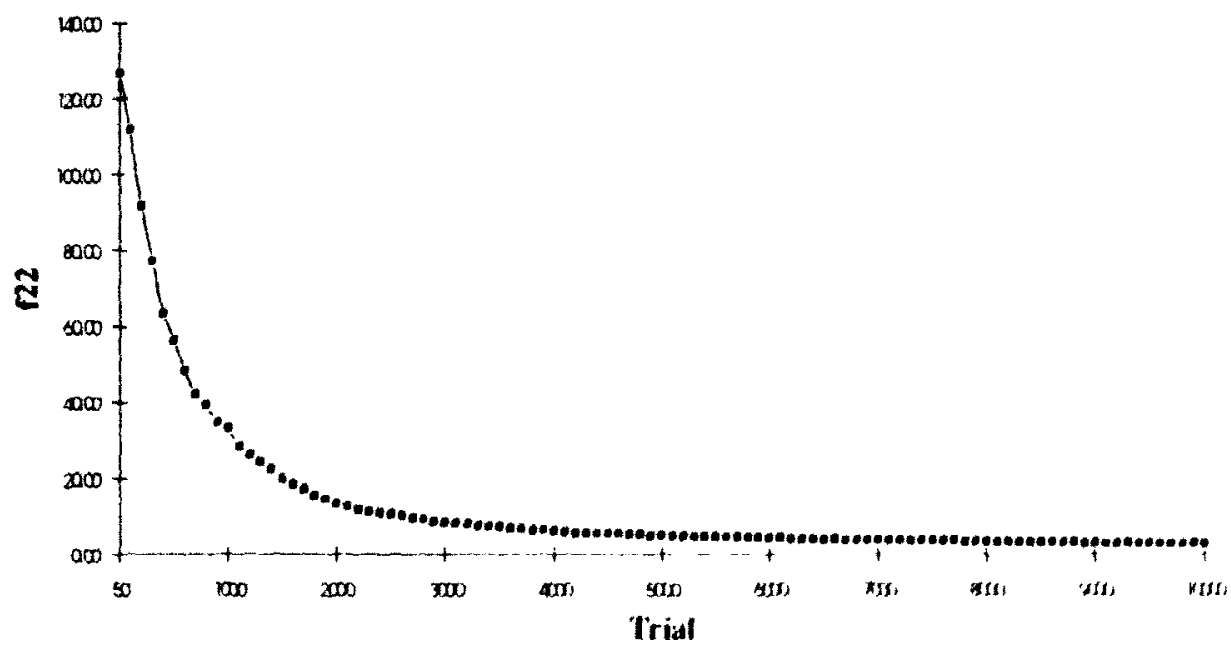

Figure $\mathscr{W} 22$ adaptive fitness 
I'hot of 222 versus 'Trial difference between adaptive and mon-adaptive runs

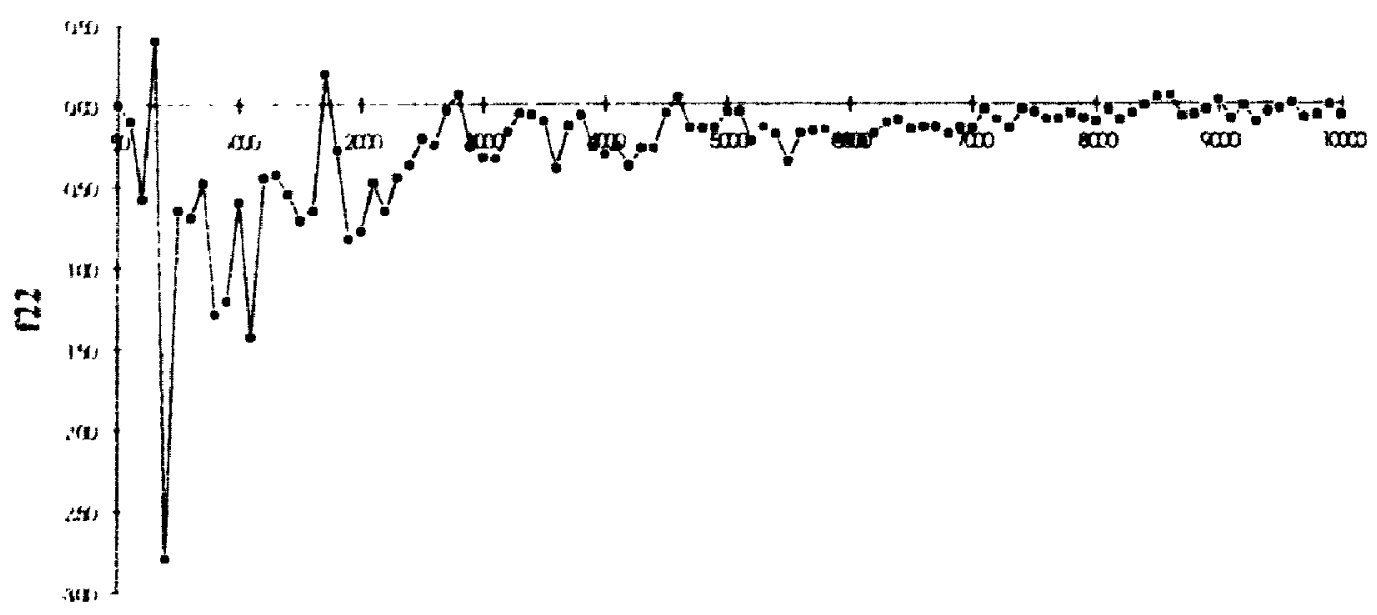

Trial

Figure 1(M) 22 adaptive - non-adaptive comparison

\section{B.1.23 Galar}

Wht of 23 versus Trial for mon-aduptive run

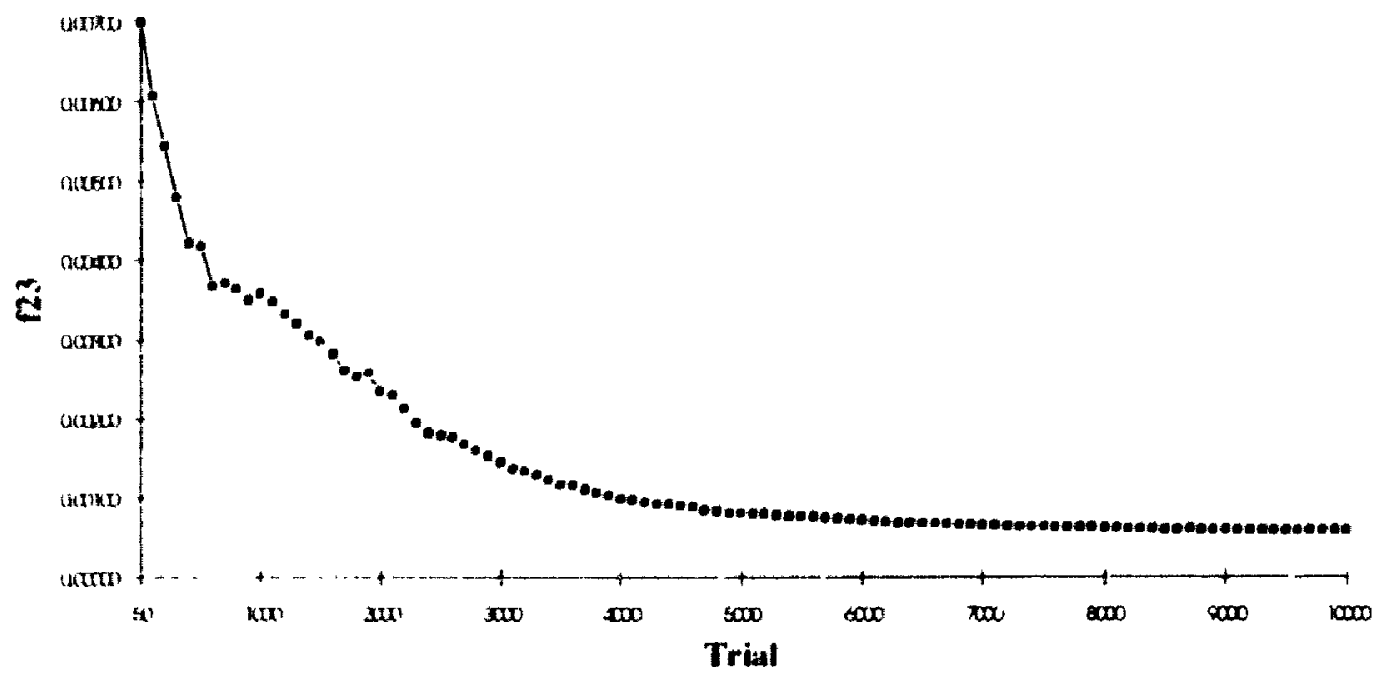

\section{Figure 10123 mon-adaptive fitness}


Appendix B: Results

Page IX X

Phot of R3 versus Trial for adaptive run

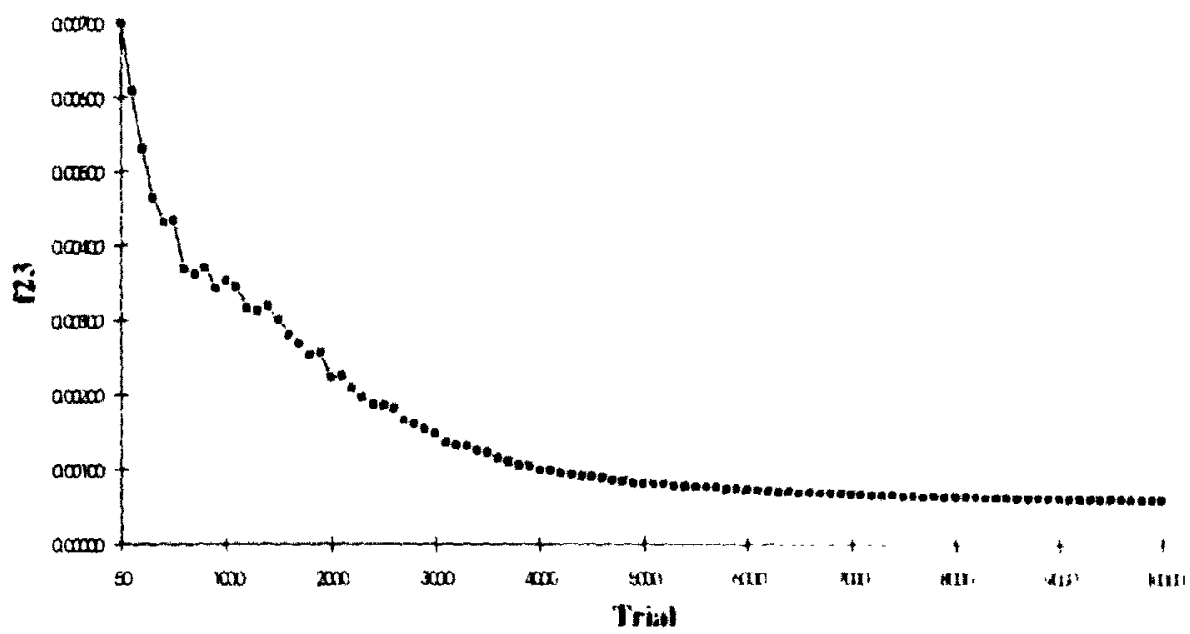

Figure $102 \mathbf{2 3}$ adaptive fitness

Pot of 2.3 versus Trial difference between adaptive and non-adaptive runs

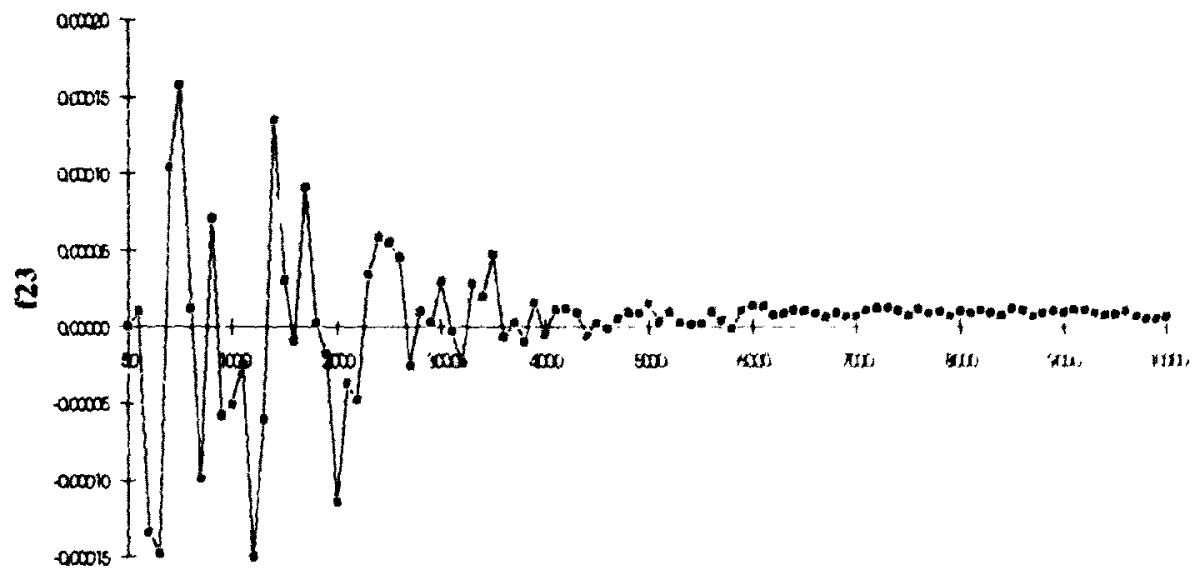

Trial

Figure 10323 adaptive - nonadaptive comparison 


\section{B.1.24 Kowalik}

Mht of 24 versus. Trial for mon-adaptive run

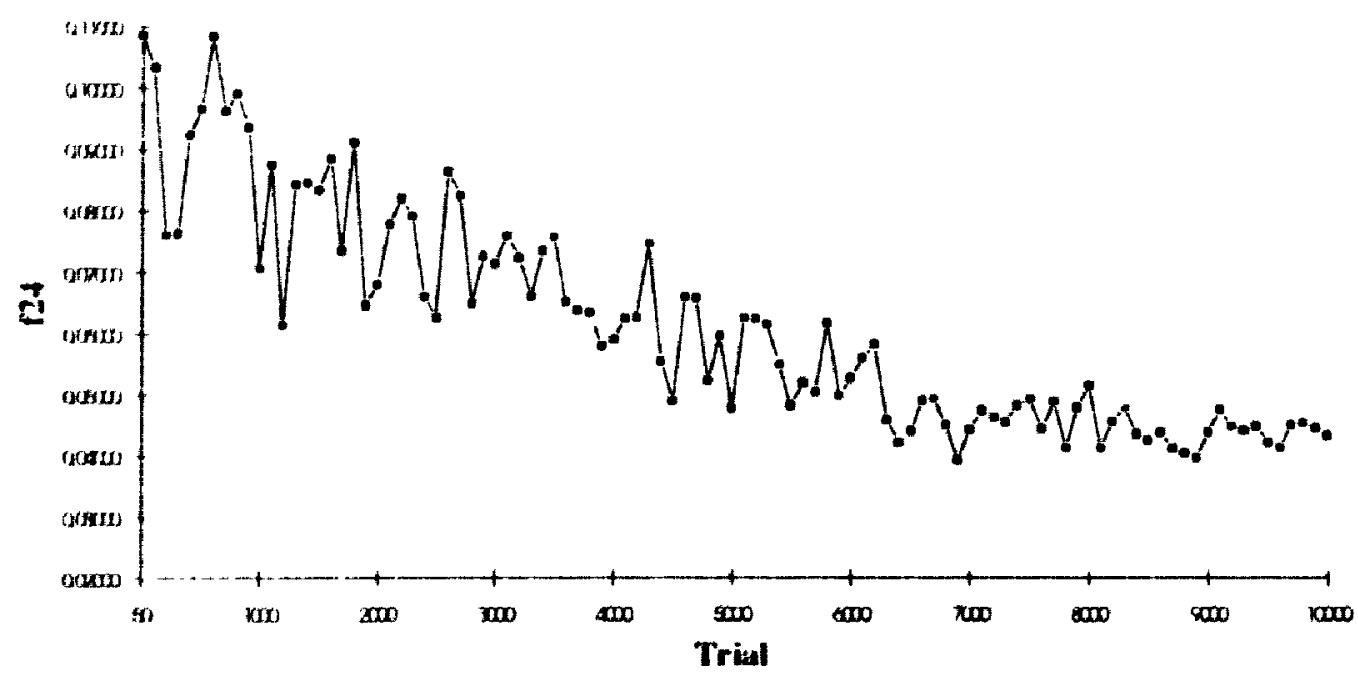

Figure 110424 mon-adaptive fitness

Mot of 24 versess Trial for adaptive run

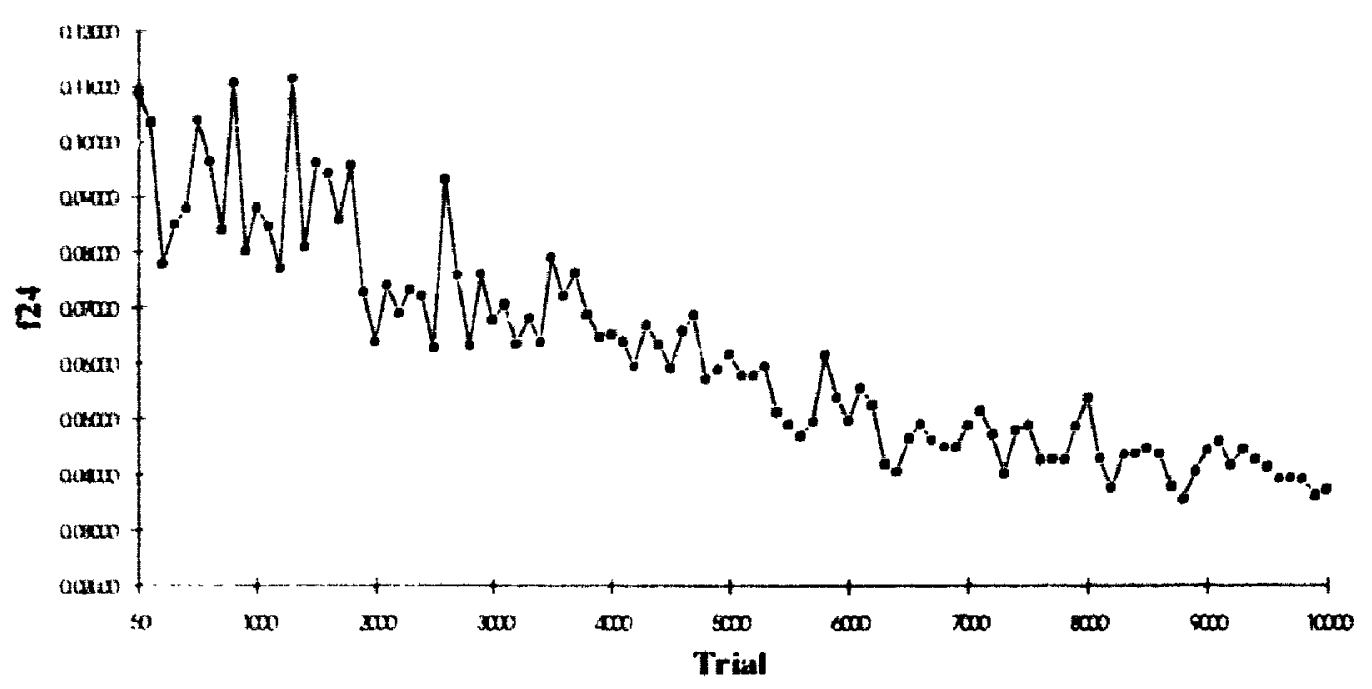

Figure 115 24 adaptive fitness 
Phot of RA versus Trial difference between adaptive and non-adaptic re rum

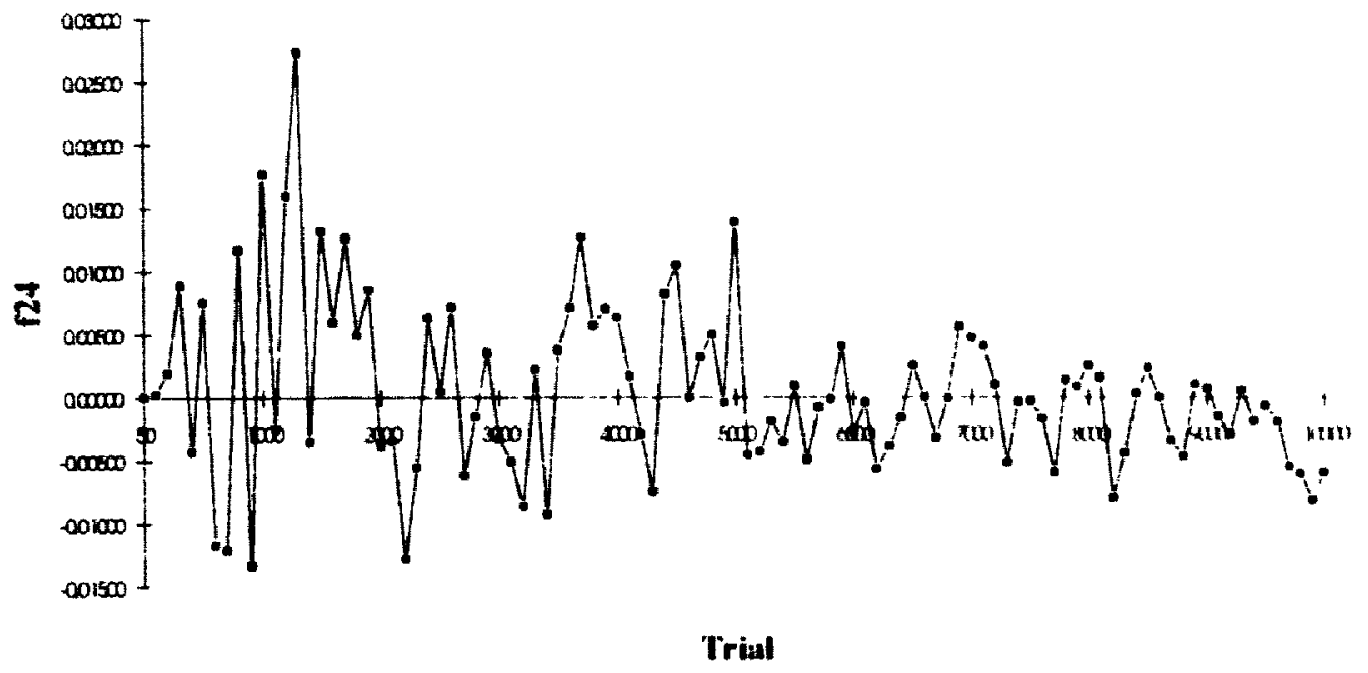

Vigure 111624 adaptive - nun-adaptive comparison 

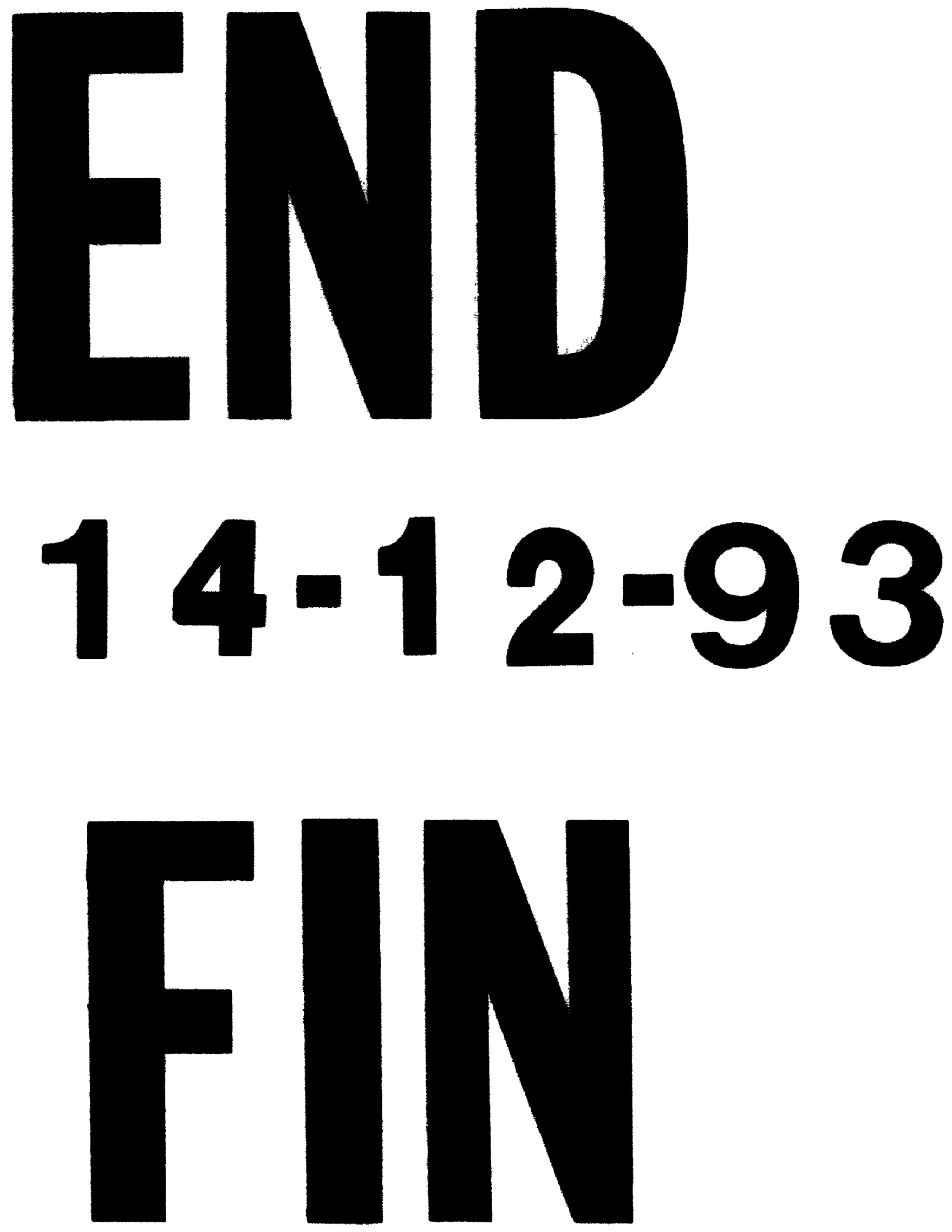